\title{
Importance of Molecular Symmetry for Enantiomeric Excess Recognition by NMR
}

\author{
Karolis Norvaiša, ${ }^{[a]}$ John E. O'Brien, ${ }^{[b]}$ Irina Osadchuk, ${ }^{[c]}$ Brendan Twamley, ${ }^{[b]}$ Victor Borovkov ${ }^{[c]}$ and \\ Mathias O. Senge ${ }^{*[a, d]}$
}

\begin{abstract}
Recently prochiral solvating agents (pro-CSA) became a spotlight for the detection of enantiopurity by NMR. Chemical shift non-equivalency in achiral hosts introduced by the presence of chiral guest yields observable resonance signal splitting $(\Delta \delta)$ correlating to the enantiomeric excess (e.e.). In this work, symmetry is our lens to explain porphyrin-based supramolecular receptors' activity in a chiral environment. Based on extensive NMR analyses of the atropisomeric receptors, host symmetry is shown to be affected by porphyrin nonplanarity and further desymmetrized in the presence of a chiral guest. We have formulated a simple, symmetry-based protocol that can be used to identify pro-CSA candidates. As such, the exposed porphyrin inner core $(\mathrm{N}-\mathrm{H})$, with its strong hydrogen bond abilities, for the first time, has been exploited in enantiomeric composition analysis. Our approach in e.e. detection by $\mathrm{N}-\mathrm{H}$ signals appearing in a previously underutilized region of the spectrum (below $0 \mathrm{ppm}$.), shows chemical shifts (the e.e. dependent splitting $\Delta \delta$ ) three times more sensitive to enantiomeric compositions than previously reported systems. The findings are complemented by extensive 2D NMR studies, including the first reporting of e.e. dependent $\Delta \delta$ in nonhydrogen NMR, and supporting by density functional theory (DFT) calculations.
\end{abstract}

Among the numerous stereodiscrimination methods, nuclear magnetic resonance (NMR) spectroscopy continues to be one of the leading tools for determining the enantiomeric purity of chiral molecules. ${ }^{[1]}$ However, enantiomers cannot be distinguished in an achiral environment as NMR active nuclei are isochronous. Usually, some external source of chirality ${ }^{[1 d]}$ is

[a] K. Norvaiša, Prof. Dr. M. O. Senge

School of Chemistry, Chair of Organic Chemistry

Trinity Biomedical Sciences Institute,

152-160 Pearse Street,

Trinity College Dublin,

The University of Dublin, Dublin 2 (Ireland)

[b] Dr. B. Twamley, Dr. J. E. O'Brien

School of Chemistry,

Trinity College Dublin

The University of Dublin, Dublin 2 (Ireland)

[c] Dr. I. Osadchuk, Prof. Dr. V. Borovkov

Department of Chemistry and Biotechnology, Tallinn University of

Technology, Akadeemia tee 15, Tallinn 12618, (Estonia)

[d] Prof. Dr. M. O. Senge

Institute for Advanced Study (TUM-IAS)

Technical University of Munich

Focus Group - Molecular and Interfacial Engineering of Organic

Nanosystems

Lichtenbergstrasse 2a, D-85748 Garching (Germany)

E-mail: mathias.senge@tum.de

twitter: @mathiassenge

www: http://www.sengegroup.eu/

Supporting information for this article is given via a link at the end of the document. introduced in the form of a covalently bonding chiral derivatization agent (CDA), a non-covalently interacting solvating agent (CSA), or even self-induced recognition of enantiomers $(\mathrm{SIRE})^{[1 \mathrm{a}]}$ to convert the enantiomeric systems into diastereomeric ones. Recently, a new type of NMR spectroscopic detection of enantiomeric excess (e.e.) using prochiral solvating agents (proCSA) was introduced by Hill and co-workers. ${ }^{[2]}$ In principle, in the event of attractive noncovalent physicochemical interactions, the chiral information of a guest can be transferred to an achiral host and detected as the splitting of the NMR signals. The key example of pro-CSA, N,N'-disubstituted oxoporphyrinogen ( $\left.\mathbf{B z}_{2} \mathbf{O x P}\right)$ exhibits a linear response between the e.e. value and the magnitude of $\beta$-proton splitting $(\Delta \delta)$ in ${ }^{1} \mathrm{H}$ NMR (Figure 1a). ${ }^{[3]}$ Due to $\mathrm{N}$-alkylation of the $\mathbf{B z}_{2} \mathbf{0 x P}$ core, the system cannot be protonated and hence suffers serious sensitivity issues compared to unmodified oxP. However, the inevitable prototropic tautomerism and macrocyclic inversions obstruct the potential applications of oxP as a pro-CSA. ${ }^{[2,4]}$ Porphyrins, as prospective pro-CSA candidates for e.e. detection, have also been investigated. ${ }^{[5]}$ While $5,10,15,20$-tetraphenylporphyrin (TPP) is not affected by the disadvantageous tautomeric processes, as opposed to $\mathbf{~ x P}$, the necessary use of depressed temperatures for the e.e. detection limits the analysis to explicit solvents with a low freezing point (e.g. $\mathrm{CDCl}_{3}$ ) and analyte solubility during the screening (e.g. precipitation).

Frequently, the use of pro-CSA's ${ }^{1} \mathrm{H}$ NMR spectra for chiral analysis is severely hampered due to the numerous scalar couplings and overlapping signals that lead to analytical difficulties. ${ }^{[6]}$ As the majority of organic molecule resonances appear between $0-14 \mathrm{ppm}$ in the ${ }^{1} \mathrm{H}$ NMR scale, ${ }^{[7]}$ it is desirable that the e.e. monitoring with pro-CSA would be in a distinct, wellseparated region. One of the most unique characteristics of porphyrins is the closed-loop of electrons (ring current) exhibiting large magnetic anisotropy under an applied magnetic field. While peripheral macrocycle signals relate to the typical organic resonances, the nuclei positioned within the loop experience a strong shielding effect when subjected to an external magnetic field and resonate below $0 \mathrm{ppm}$ in the ${ }^{1} \mathrm{H}$ NMR scale. ${ }^{[8]}$ Once the highly conjugated system is disrupted (e.g., in oxopophyrinogens, calix[4]pyrroles), the anisotropic shielding effect of the inner core system is lost, resulting in downfield shifting of the corresponding inner core signals.

The attractive features of the metal-free (free base) porphyrin inner core has lately drawn attention in the fields of catalysis $^{[9]}$, sensing ${ }^{[10]}$, supramolecular assemblies ${ }^{[11]}$, and absolute configuration determination. ${ }^{[12]}$ Typically, the imine and amine units of porphyrins are not involved in intermolecular interactions due to the planar nature of the macrocycle. However, the existing methods of ring puckering by steric strain ${ }^{[13]}$ can cause a degree of outwards orientation of the inner pyrrolic entities, making these positions more basic ${ }^{[14]}$ and accessible to 
substrates. ${ }^{[10]}$ Even though porphyrins adopt a saddle-shaped 3D conformation $^{[15]}$ creating an 'active center' in the core, only the saddle-deformation alongside chiral guest interactions is not enough to drive the inner $\mathrm{N}-\mathrm{H}$ signal to split during the ${ }^{1} \mathrm{H}$ NMR e.e. analysis. For example, $\mathbf{B z}_{2} \mathbf{O x P}$ has a saddle shape and belongs to the $C_{2 v}$ point-group notation with two mirror planes diagonally dividing all pyrroles (Figure 1a). The symmetrical nature of $\mathbf{B z _ { 2 }} \mathbf{O x P}$ does not permit the e.e. discrimination using the inner core. However, the $\mathrm{N}-\mathrm{H}$ signal shifts to the lower field of the spectrum due to the non-covalent interactions nevertheless remain isochronous. ${ }^{[3]}$

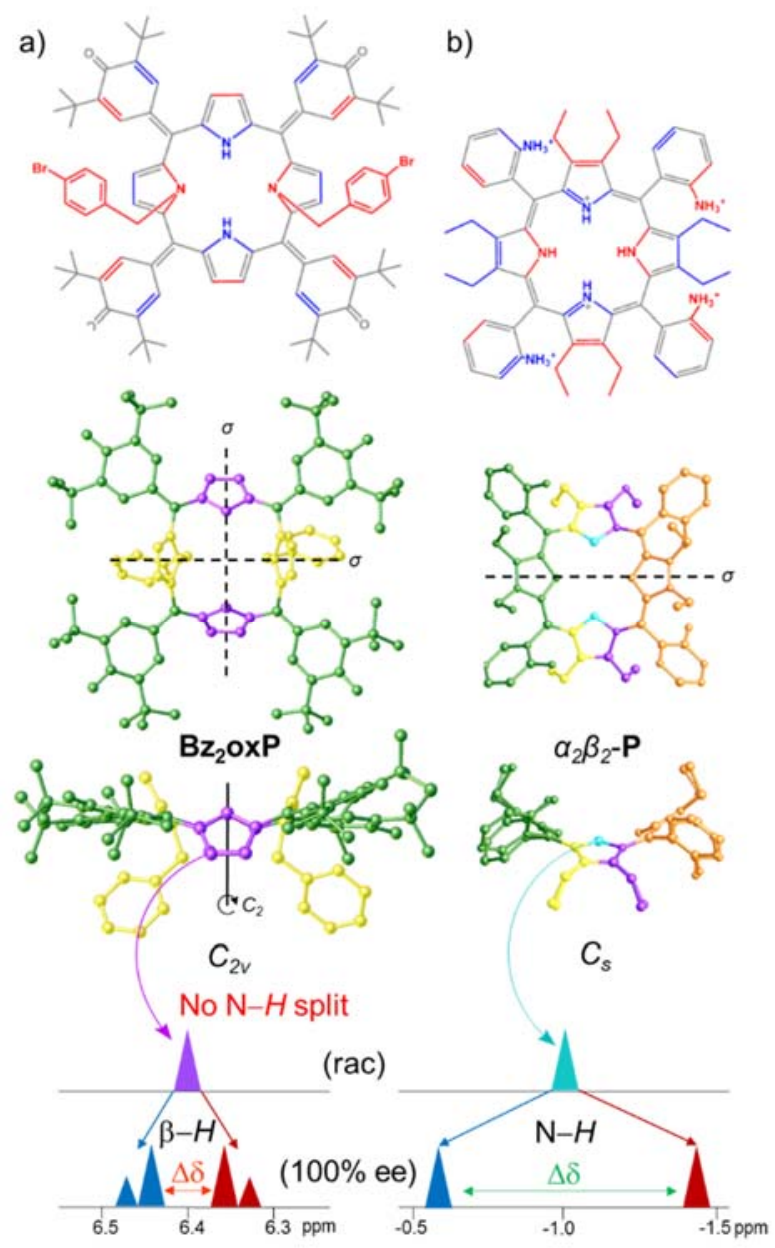

Figure 1. Top: representation of pro-CSA's (blue above the plane, red below); Middle: with symmetry elements (Mirror plane $\sigma$ and rotation axis $C_{n}$ ); Bottom: the key units used for e.e. detection by ${ }^{1} \mathrm{H}$ NMR; a) $\mathbf{B} \mathbf{z}_{2} \mathbf{0 x} \mathbf{P}$ highlighting $\beta-H$ splitting; ${ }^{[3]}$ b) newly designed $\alpha_{2} \beta_{2}-\mathbf{P}$ receptor system with chiral discrimination by $\mathrm{N}-\mathrm{H}$; c) All possible $\mathbf{P}$ atropisomers with corresponding point groups, $\mathrm{N}-\mathrm{H}$ signals, and magnitude of splitting; see more detail in Figure $\mathrm{S} 1$.

Here we report the first example of e.e. detection using porphyrin inner core $\mathrm{N}-\mathrm{H}$ resonances. We have designed $\mathbf{P}$ $[5,10,15,20$-tetrakis(2-aminiumphenyl)-2,3,7,8,12,13,17,18-

octaethylporphyrin] as a receptor system (Figure 2a) exploiting three main molecular engineering strategies: 1) steric overcrowding to obtain a saddle-shaped macrocycle while retaining the porphyrin conjugation ${ }^{[13]}$ and exposing the inner pyrrolic units for host-guest interactions; 2) peripheral donating groups creating a lock-and-key ${ }^{[10 c]}$ comparable system to encapsulate chiral analytes in the porphyrin lattice and allow the detailed NMR analysis at room temperature. ${ }^{[10 \mathrm{~d}]} 3$ ) formation of atropisomers based on the orientation of peripheral groups ${ }^{[16]}$ to have the ultimate control of the symmetry elements in pro-CSA. ${ }^{[17]}$

In our previous study, we have highlighted the selective nature of host $\mathbf{P}$ for guests containing sulfonate or phosphonate motifs. ${ }^{[10 \mathrm{~d}]}$ The analyte interacts directly with the inner ring system and generates static and well-resolved NMR spectral lines. As previously mentioned, the depressed temperatures can also offer slow exchange rates for potential detection of e.e. ${ }^{[5]}$ However, the aim of the following studies is the development of a readily available and highly effective analytical tool for room-temperature measurements. Therefore, ( \pm )-10-camphorsulfonic acid (10CSA) bearing the sulfonic moiety and stereogenic centers was selected as a chiral guest in the present study.

Operating with enantiopure $\mathbf{1 0 C S A}(\mathbf{S}$ or $\mathbf{R})$ four distinct scenarios with four different $\mathbf{P}$ atropisomers were observed and subsequently rationalized by the symmetry operations found in $\mathbf{P}$ (Figure 1c). ${ }^{[17]}$ In the $\alpha_{4}-\mathbf{P} \cdot 10 \mathrm{CSA}(\mathbf{S}$ or $\mathbf{R})$ complex, the inner core remains isochronous, due to the $C_{2 v}$ point-group notation with a two-fold symmetry axis and two mirror planes passing through the pyrroles. The identical situation previously reported by Hill and coworkers in $\mathbf{B z}_{2} \mathbf{0 x} \mathbf{P}$ pinpoints the interactions with inner $\mathrm{N}-\mathrm{H}$, however, without the e.e. discrimination due to the $C_{2 v}$ symmetry (Figure 1a). ${ }^{[3]}$ The $\alpha_{2} \beta_{2}-\mathbf{P}$ atropisomer with $C_{s}$ symmetry features a single well-defined mirror plane dividing two pyrrolic units which preserve its achiral nature, hence allowing it to be classified as pro-CSA. The lack of other symmetry elements in $\alpha_{2} \beta_{2}-\mathbf{P}$ allows the $\mathrm{N}-\mathrm{H}$ protons to become anisochronous in a chiral environment, making chiral discrimination possible (with the highest magnitude of splitting ( $\Delta \delta_{\max }$ ) of $0.653 \mathrm{ppm}$ at $100 \%$ e.e.) (Figure $1 \mathrm{~b}$ ). The $\alpha_{3} \beta-\mathbf{P}$ atropisomer belongs to the $C_{1}$ point-group, as it contains no symmetry elements, making the system chiral. Thus, eight signals are observed with enantiopure 10CSA due to diastereomer formation ( $S S$ - and $S R$ - or $R R$ - and $R S$-) (Figure S1). While the e.e. detection is possible with $\alpha_{3} \beta-P$ (Figure S2), the practical use of such system falls short mainly due to three dominating factors: 1) the high number of inner core system signals hampers direct e.e. interpretation; 2) the magnitude of $\Delta \delta_{\max }(\sim 0.39 \mathrm{ppm})$ is lowest of the three atropisomers with inner core splitting making it the least sensitive system; 3 ) the concentration of $\alpha_{3} \beta-\mathbf{P}$ is required to be significantly higher than that of other systems due to a large number of resonance signals and their comparatively lower intensities. On the other hand, $\alpha \beta \alpha \beta$-P which belongs to the $S_{4}$ point group has four equivalent protons located in the principal axis. While it has no mirror planes, the inversion center situated between the pyrrole units allows the inner core protons to split in equal proportions (above and below the plane) upon interaction with a chiral analyte. A single isochronous $\mathrm{N}-\mathrm{H}$ signal of $\alpha \beta \alpha \beta-\mathbf{P}-10 \operatorname{CSA}(\mathbf{S}$ or $\mathbf{R})$ becomes 

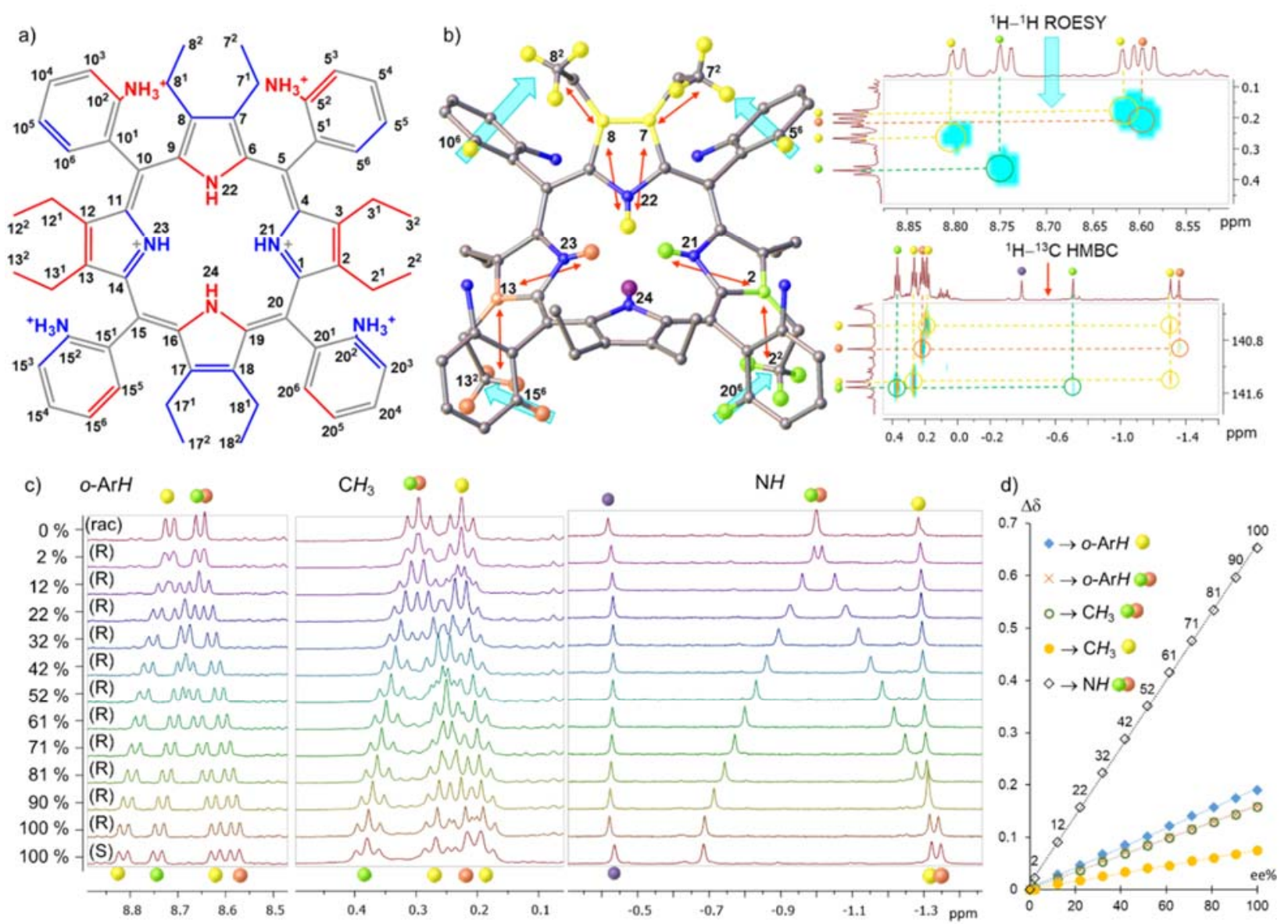

Figure 2. a) Illustration of the structure of $\alpha_{2} \beta_{2}-\mathbf{P}$ (blue - above and red - below the plane) with corresponding positions; b) representation and color-coding reference of the ${ }^{1} \mathrm{H}$ splitting signals with blue arrows showing ${ }^{1} \mathrm{H}-{ }^{1} \mathrm{H}$ ROESY and red arrows ${ }^{1} \mathrm{H}-{ }^{13} \mathrm{C} \mathrm{HMBC}$ correlations of 20 eq. $\alpha_{2} \beta_{2}-\mathbf{P} \cdot 10 \mathrm{CSA}(\mathbf{R})$; C) Observable $\Delta \sigma$ of ${ }^{1} \mathrm{H}$ signals in o-ArH, $\mathrm{CH}_{3}$, and inner core system $(\mathrm{NH})$ regions; d) Graph of the $\Delta \sigma$ dependence on the e.e.\% value. All spectra have been recorded in $\mathrm{CD}{ }_{3} \mathrm{CN}$.

anisochronous, with $\Delta \delta_{\max }(0.520 \mathrm{ppm})$ comparable to the $\alpha_{2} \beta_{2^{-}}$ $\mathbf{P} \cdot 10 \mathrm{CSA}(\mathbf{S}$ or $\mathbf{R})$ system (0.653 ppm). While a singular inner core proton splitting is an attractive feature, the practicality of such a system in the e.e. detection is challenging, mainly due to the low atropisomeric rotational barrier, which leads to the formation of other atropisomers at room temperature ${ }^{[10 \mathrm{~d}]}$ and low abundancy (only $1 / 8$ obtained from statistical mixtures) in comparison to other $\mathbf{P}$ rotamers. Since $\alpha_{2} \beta_{2}-\mathbf{P}$ displayed the highest $\Delta \sigma_{\max }$ value compared to other $\mathbf{P}$ atropisomeric species (Figure 1c), in-depth chirality determination studies listed below were carried out with this receptor system.

Overall, three distinct and well-resolved regions $\left(\mathrm{o}-\mathrm{ArH}, \mathrm{CH}_{3}\right.$, and $\mathrm{N}-H$ ) were identified for possible e.e. monitoring with $\alpha_{2} \beta_{2}-\mathrm{P}$ (Figure 2c, S5). The correlation between the signals of interest was investigated by 2D NMR techniques with enantiopure 10CSA(R) (20 eq.) and their corresponding locations are illustrated in Figure 2b. The gradual addition of 10CSA(R) to $\alpha_{2} \beta_{2^{-}}$ $\mathbf{P}$ and the influence of water on $\Delta \sigma_{\max }$ as a competitive agent is detailed in the SI (Figure S3-S9). While the $\Delta \sigma_{\max }$ values of o-ArH and $\mathrm{CH}_{3}$ are comparable to known pro-CSAs ${ }^{[2-5,18]}$ being 0.190 ppm (o-ArH yellow), $0.159 \mathrm{ppm}$ (o-ArH red/green), $0.158 \mathrm{ppm}$ $\left(\mathrm{CH}_{3}\right.$ red/green $)$, and $0.075 \mathrm{ppm}\left(\mathrm{CH}_{3}\right.$ yellow $)$, the $\Delta \sigma_{\max }$ values of the inner system ( $\mathrm{N}-\mathrm{H}$ red/green) was found to be more than threefold greater than those of other regions $(0.653 \mathrm{ppm})$.

The origin of the chemical shift non-equivalence lies deep within the concept of prochirality. ${ }^{[19]}$ The desymmetrization of $\alpha_{2} \beta_{2}$-P atoms in a single step by weak interactions with a chiral guest proves to be particularly useful for the e.e. determination. To illustrate 10CSA interactions with $\alpha_{2} \beta_{2}-\mathbf{P}$, a conformational search was performed using the $\alpha_{2} \beta_{2}-\mathrm{P}\left[\mathrm{SO}_{4}{ }^{2-}\right]\left[\mathrm{HSO}_{4}{ }^{-}\right]_{4}$ structure for building starting geometries. Corresponding noncovalent interactions of the major conformer are illustrated in Figure S14. When racemic and non-racemic mixtures of 10CSA were applied in the system at constant concentrations it was found that $\Delta \sigma$ of the o-ArH, $\mathrm{CH}_{3}$, and $\mathrm{N}-\mathrm{H}$ peaks rely on respective $\%$ of the e.e. value (Figure $2 \mathrm{c}$ ). At the racemate point, the isochronous profile of $\alpha_{2} \beta_{2}-\mathbf{P}$ is restored since the chiral information is transmitted in equal proportions from both the chiral components. Since the $\Delta \sigma_{\max }$ value of the inner core system is substantially higher than that of other regions, the resolution, of which e.e. can be detected, is considerably enhanced. Astonishingly, at as low as $2 \%$ e.e., two distinct $\mathrm{N}-\mathrm{H}$ resonance singlets $(\Delta \sigma 0.022 \mathrm{ppm}$.) can clearly be identified, while the other regions show only a broadening of the signals. Plotting the differences in the chemical shifts of split 
peaks against the \% e.e. values revealed a linear dependency with the $\mathrm{R}^{2}$ values being above 0.997 and the inner $\mathrm{N}-\mathrm{H}$ fitting $\mathrm{R}^{2}$ $=0.9994$ (Figure 2d). The linear fit of the plots is a fundamental property in unlocking the easy calibration of the referenced systems for quick detection of the e.e. value (a detailed example shown in SI; Figure S10-S12). Moreover, spatially distant neighboring protons from $\mathrm{N}-\mathrm{H}$ offer another important feature. Sharp and well-isolated singlets do not suffer from any vicinal scalar J-couplings or roofing effects underlining the simplicity in tracking chiral compositions. Overall, monitoring changes of this inner core system in a model chiral environment demonstrates a powerful tool for easy and sensitive detection of enantiomeric compositions.

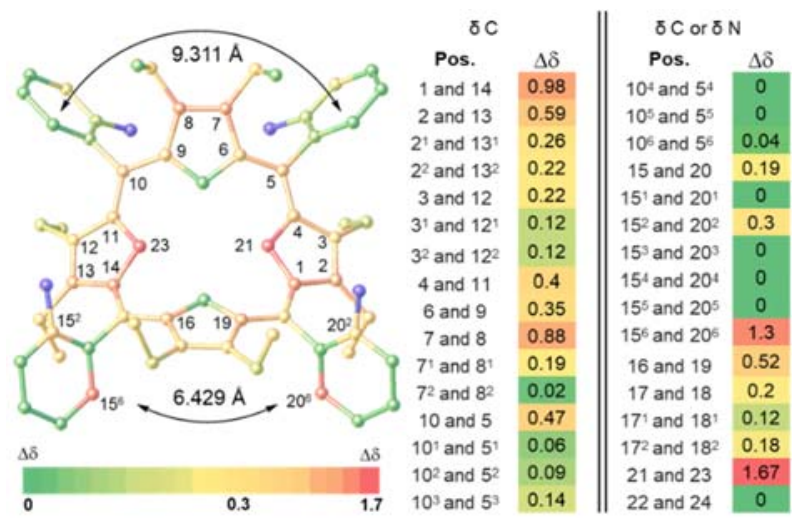

Figure 3. Illustration of $\Delta \sigma_{\max }(\mathrm{ppm})$ of ${ }^{13} \mathrm{C}$ and ${ }^{15} \mathrm{~N}$ NMR in 20 eq. $\alpha_{2} \beta_{2}-\mathrm{P}$ 10CSA(S) complex, determined in comparison to the corresponding racemate $\alpha_{2} \beta_{2}$-P-10CSA(SR) using 2D NMR techniques $\left(\mathrm{CD}_{3} \mathrm{CN}\right)$ (Figure S16-S31). The highlighted positions in the illustration on the left side shows $\Delta \sigma \geq 0.3 \mathrm{ppm}$. Atoms in blue are peripheral nitrogen atoms that did not resonate.

The magnitude of non-hydrogen $\Delta \sigma$ relies on the spatial positions, distances, and interactions with chiral guests. ${ }^{[3]}$ The further the stereogenic center of a chiral guest from the host molecule, the weaker the chirality transfer is. Interestingly, this principle was previously well-defined in the porphyrin-based hostguest chirogenic systems by using circular dichroism spectroscopy. ${ }^{[20]}$

The dependence of the non-equivalency to the chiral guest location can be illustrated by ${ }^{13} \mathrm{C}$ and ${ }^{15} \mathrm{~N}$ NMRs. (For detailed comparison of non-hydrogen resonances see Table S1). When $\alpha_{2} \beta_{2}-\mathbf{P} \cdot \mathbf{1 0 C S A}(\mathbf{S})$ was compared to racemic $\alpha_{2} \beta_{2}-\mathbf{P} \cdot \mathbf{1 0 C S A}(\mathbf{S R})$, most of the macrocyclic ring system exhibited $\Delta \sigma_{\max }>0.3 \mathrm{ppm}$ with the central two nitrogen atoms having $\Delta \sigma_{\max }=1.67 \mathrm{ppm}$ (Figure 3). Nevertheless, due to the greater distance from the active site, most of the phenyl ring resonance signals remained isochronous. Despite this, two particularly different scenarios were portrayed by the $0-A r-{ }^{13} \mathrm{C}$ NMR signals. The $\Delta \sigma_{\max }$ between $15^{6}$ and $20^{6}$ positions yielded excellent separation $(\sim 1.3 \mathrm{ppm})$, whereas the $5^{6}$ and $10^{6}$ imposed only marginal $\Delta \sigma_{\max }(0.04 \mathrm{ppm})$. A closer examination of the crystal structure of $\alpha_{2} \beta_{2^{-}}$ $\mathrm{P}\left[\mathrm{SO}_{4}{ }^{2}\right]\left[\mathrm{HSO}_{4}{ }^{-}\right]_{4}$ revealed a closer distance between $\mathrm{C} 15^{6}$ and $\mathrm{C} 20^{6}(\sim 6.429 \AA)$ than between $\mathrm{C}^{6}$ and $\mathrm{C} 10^{6}(\sim 9.311 \AA)$, subsequently forming a narrow channel for the chiral guest to occupy (Figure 3 ). Moreover, the calculated chemical shifts of non-hydrogen atoms in $\alpha_{2} \beta_{2}-\mathbf{P} \cdot \mathbf{1 0 C S A}(\mathbf{R})$ using the GIAOB3LYP/6-311++G**/BP86-D3BJ/def-SVP method and SMD solvent model correlated well with the splitting patterns observed experimentally (for more information check SI, Table S8). Of note, in ${ }^{1} \mathrm{H}$ NMR the $15^{6}$ and $20^{6}$ positions had similar $\Delta \sigma_{\max }$. Hence, while hydrogens of the $\mathrm{o}-\mathrm{Ar}$ group were more likely to form weak interactions with the chiral compounds, the corresponding $\Delta \sigma_{\max }$ value of carbon resonances hinges on the spatial arrangements and proximity to the guest. Comparison of the $\alpha_{2} \beta_{2}-\mathbf{P} \cdot 10 \mathrm{CSA}$ (S and SR) splitting resonance signals to other atropisomeric species is detailed in SI (Table S2-S3).

To conclude, the point groups were found to play a fundamental role in adjusting supramolecular receptor systems for e.e. determinations by the NMR method. Four atropisomers containing different point group notations were thoroughly investigated by NMR with ( $S$ and $R$ ) camphorsulphonic acid pinpointing the $\alpha_{2} \beta_{2}$ rotamer as the most sensitive receptor for chirality detection. It was found that the $\Delta \sigma_{\max }$ value of $\mathrm{N}-\mathrm{H}$ signals can reach $0.653 \mathrm{ppm}$, a three-fold greater splitting than any known pro-CSA. Such enhanced sensitivity towards the chiral components allows for readily available and exceptionally detailed enantiomeric excess detection at room temperature by NMR.

Deposition number 2143572 (for $\left.\alpha_{2} \beta_{2}-\mathrm{P}_{\mathrm{SO}_{4}}{ }^{2}\right]\left[\mathrm{HSO}_{4}{ }^{-}\right]_{4}$ ) contains the supplementary crystallographic data for this paper. These data are provided free of charge by the joint Cambridge Crystallographic Data Centre and Fachinformationszentrum Karlsruhe http://www.ccdc.cam.ac.uk/structures Structures service.

\section{Acknowledgements}

This work was prepared with the support of the Technical University of Munich - Institute for Advanced Study through a Hans Fischer Senior Fellowship and has received funding from the European Union's Horizon 2020 research and innovation program under the FET-OPEN grant agreement No.828779, the Irish Research Council (GOIPG 2017/1172), Science Foundation Ireland (IvP 13/IA/1894) and was supported by the Higher Education Authority and the Department of Further and Higher Education, Research, Innovation and Science (Ireland).

\section{Conflict of Interest}

The authors declare no conflict of interest.

Keywords: Enantiomeric Excess • Porphyrinoids • Atropisomers -NMR • Symmetry Elements

[1] a) Z. Szakács, Z. Sánta, A. Lomoschitz, C. Szántay, TrAC, Trends Anal. Chem. 2018, 109, 180-197; b) M. S. Silva, Molecules 2017, 22, 247268; c) J. S. Fossey, E. V. Anslyn, W. D. G. Brittain, S. D. Bull, B. M. Chapin, C. S. Le Duff, T. D. James, G. Lees, S. Lim, J. A. C. Lloyd, C. V. 
Manville, D. T. Payne, K. A. Roper, J. Chem. Educ. 2017, 94, 79-84; d) T. J. Wenzel, J. D. Wilcox, Chirality 2003, 15, 256-270.

[2] a) J. Labuta, J. P. Hill, S. Ishihara, L. Hanyková, K. Ariga, Acc. Chem Res. 2015, 48, 521-529; b) A. Shundo, J. Labuta, J. P. Hill, S. Ishihara, K. Ariga, J. Am. Chem. Soc. 2009, 131, 9494-9495.

[3] J. Labuta, S. Ishihara, T. Šikorský, Z. Futera, A. Shundo, L. Hanyková, J. V. Burda, K. Ariga, J. P. Hill, Nat. Commun. 2013, 4, 2188-2196.

[4] a) J. Labuta, S. Ishihara, K. Ariga, J. Hill, Symmetry 2014, 6, 345-367; b) J. Labuta, Z. Futera, S. Ishihara, H. Kourilova, Y. Tateyama, K. Ariga, J. P. Hill, J. Am. Chem. Soc. 2014, 136, 2112-2118.

[5] a) J. Labuta, S. Ishihara, J. P. Hill, J. Porphyr. Phthalocyanines 2020, 24, 320-329; b) J. Labuta, S. Ishihara, A. Shundo, S. Arai, S. Takeoka, K. Ariga, J. P. Hill, Chem. Eur. J. 2011, 17, 3558-3561.

[6] J. Labuta, S. Ishihara, D. T. Payne, K. Takimoto, H. Sato, L. Hanyková, K. Ariga, J. P. Hill, Chemosensors 2021, 9, 259-276.

[7] a) G. R. Fulmer, A. J. M. Miller, N. H. Sherden, H. E. Gottlieb, A. Nudelman, B. M. Stoltz, J. E. Bercaw, K. I. Goldberg, Organometallics 2010, 29, 2176-2179; b) M. Balci, in Basic 1H- and 13C-NMR Spectroscopy (Ed.: M. Balci), Elsevier Science, Amsterdam, 2005, pp. 25-85.

[8] J. E. Falk, in Porphyrins and Metalloporphyrins (Ed.: K. M. Smith), Elsevier Scientific Pub. Co, Amsterdam 1975, pp. 399-514.

[9] a) M. Roucan, M. Kielmann, S. J. Connon, S. S. R. Bernhard, M. O. Senge, Chem. Commun. 2018, 54, 26-29; b) E. Aoki, W. Suzuki, H. Kotani, T. Ishizuka, H. Sakai, T. Hasobe, T. Kojima, Chem. Commun. 2019, 55, 4925-4928; c) T. A. Dar, B. Uprety, M. Sankar, M. R. Maurya, Green Chem. 2019, 21, 1757-1768; d) M. Kielmann, N. Grover, W. W. Kalisch, M. O. Senge, Eur. J. Org. Chem. 2019, 2448-2452; e) W. Suzuki, H. Kotani, T. Ishizuka, T. Kojima, J. Am. Chem. Soc. 2019, 141, 59875994.

[10] a) N. Chaudhri, M. Sankar, RSC Adv. 2015, 5, 3269-3275; b) R. Kumar, N. Chaudhri, M. Sankar, Dalton Trans. 2015, 44, 9149-9157; c) M. Kielmann, M. O. Senge, Angew. Chem. Int. Ed. 2019, 58, 418-441; d) K Norvaiša, K. J. Flanagan, D. Gibbons, M. O. Senge, Angew. Chem. Int. Ed. 2019, 58, 16553-16557; e) K. Norvaiša, M. Kielmann, M. O. Senge, ChemBioChem 2020, 21, 1793-1807.

[11] a) C. J. Kingsbury, K. J. Flanagan, H.-G. Eckhardt, M. Kielmann, M. O. Senge, Molecules 2020, 25, 3195-3218; b) K. Norvaiša, K. Yeow, B. Twamley, M. Roucan, M. O. Senge, Eur. J. Org. Chem. 2021, 1871-1882.

[12] P. Bhyrappa, V. V. Borovkov, Y. Inoue, Org. Lett. 2007, 9, 433-435.

[13] a) M. O. Senge, in The Porphyrin Handbook, Vol. 1 (Eds.: K. M. Kadish, K. M. Smith, R. Guilard), Academic Press, 2000, pp. 239-347; b) M. O. Senge, Chem. Commun. 2006, 243-256.

[14] O. S. Finikova, A. V. Cheprakov, P. J. Carroll, S. Dalosto, S. A. Vinogradov, Inorg. Chem. 2002, 41, 6944-6946.

[15] C. J. Kingsbury, M. O. Senge, Coord. Chem. Rev. 2021, 431, 213760.

[16] K. Norvaisa, S. Maguire, C. Donohoe, J. E. O'Brien, B. Twamley, L. C. Gomes-da-Silva, M. O. Senge, Chem. Eur. J., Accepted Author Manuscript, DOI: 10.1002/chem.202103879.

[17] K. Norvaiša, J. E. O'Brien, D. J. Gibbons, M. O. Senge, Chem. Eur. J. 2020, 27, 331-339.

[18] a) S. Ishihara, J. Labuta, Z. Futera, S. Mori, H. Sato, K. Ariga, J. P. Hill, J. Phys. Chem. B 2018, 122, 5114-5120; b) K. Takimoto, S. Ishihara, J. Labuta, V. Březina, D. T. Payne, J. P. Hill, K. Ariga, M. Sumita, S. Mori, H. Sato, J. Phys. Chem. Lett. 2020, 11, 8164-8169.

[19] P. Lesot, C. Aroulanda, H. Zimmermann, Z. Luz, Chem. Soc. Rev. 2015, 44, 2330-2375.

[20] V. V. Borovkov, J. M. Lintuluoto, Y. Inoue, J. Am. Chem. Soc. 2001, 123, 2979-2989. 


\section{Table of Contents}

\section{COMMUNICATION}

Just like a screwdriver turning screws, symmetry elements can be adjusted by fine-tuning the orientation of rotationally restricted side-groups in supramolecular receptors. In this research we highlight the fundamental role of symmetry in chiral reporting by NMR. Newly designed porphyrins with exposed inner core $\mathrm{N}-\mathrm{H}$ system can respond to a chiral guest with exceptionally sensitive enantiomeric excess detection.

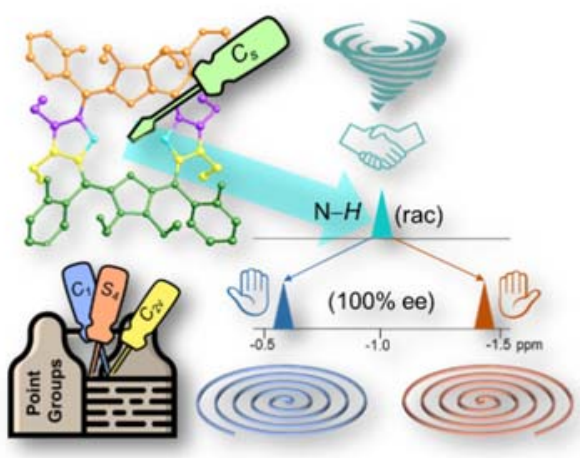

Karolis Norvaiša, John E.

O'Brien, Irina Osadchuk, Brendan

Twamley, Victor Borovkov and

Mathias O. Senge

Importance of Molecular

Symmetry for Enantiomeric

Excess Recognition by NMR 
Supporting Information 


\section{SUPPORTING INFORIMATION}

\section{Table of Contents}

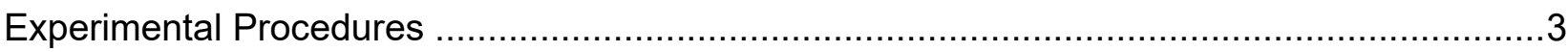

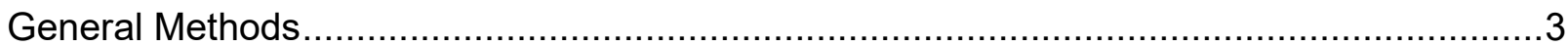

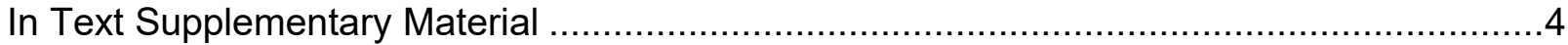

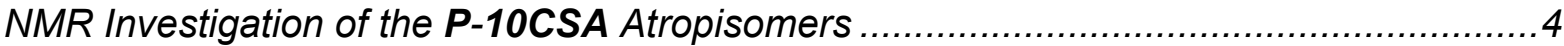

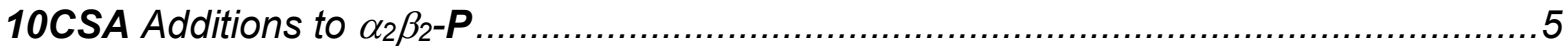

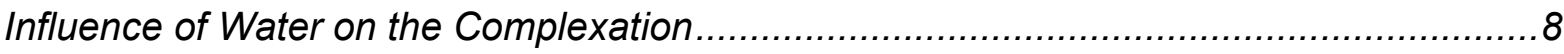

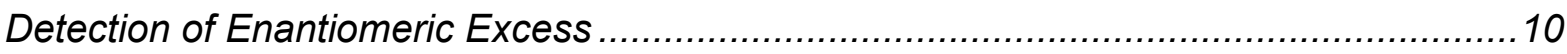

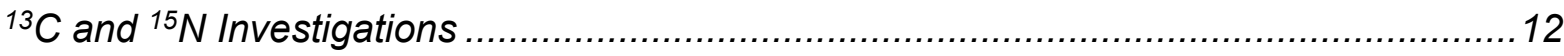

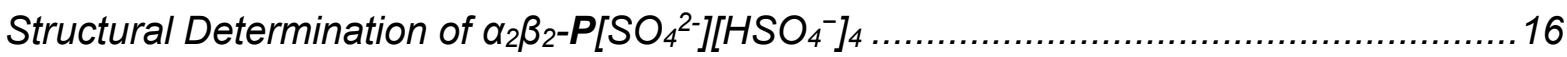

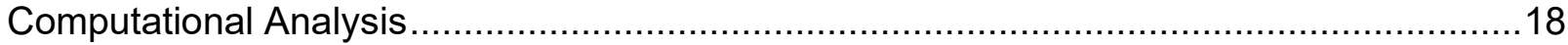

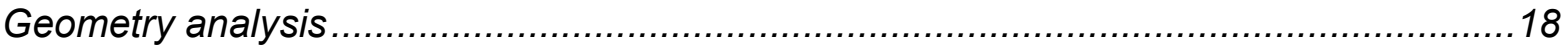

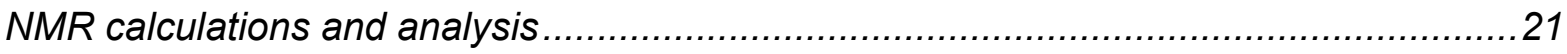

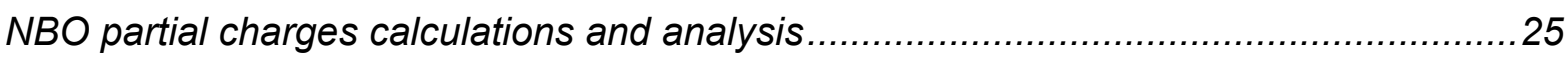

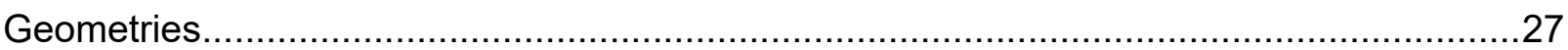

Conformer $\boldsymbol{A}$

Conformer $\boldsymbol{B}$

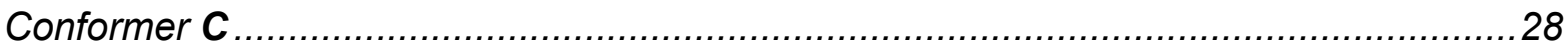

Conformer $\boldsymbol{D}$

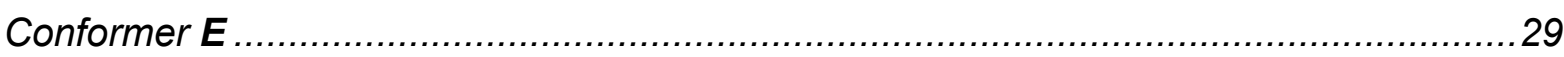

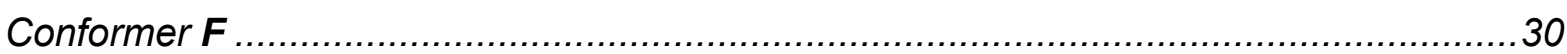

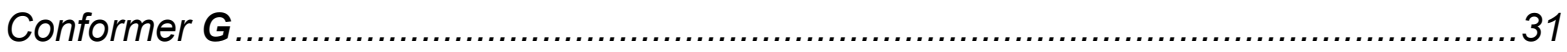

Conformer $\boldsymbol{H}$

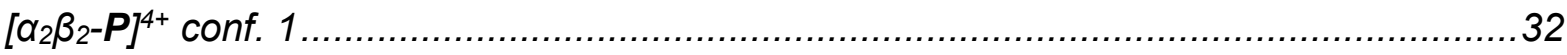

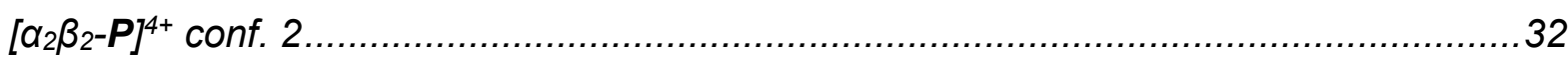

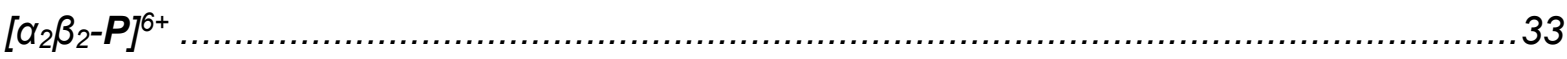

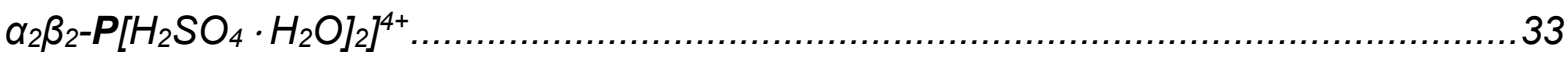

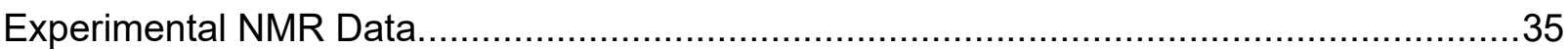

$\alpha_{2} \beta_{2}-P$-10CSA(S)

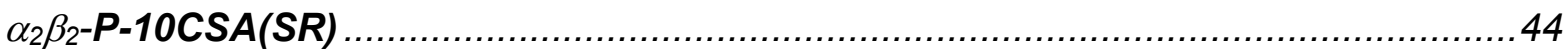

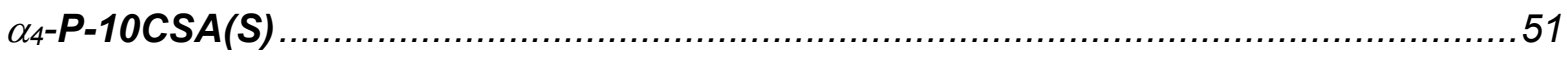

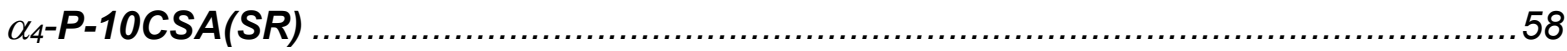

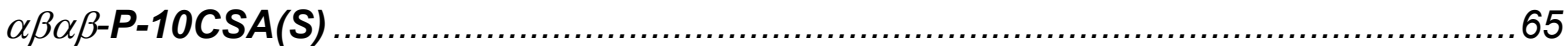

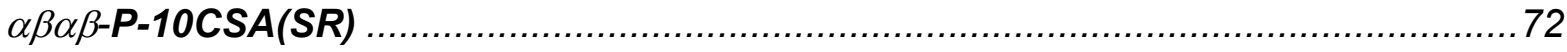

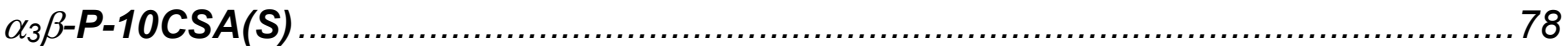

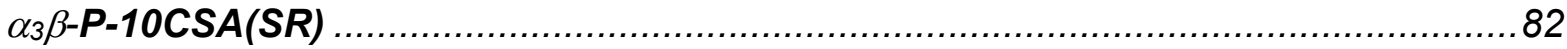

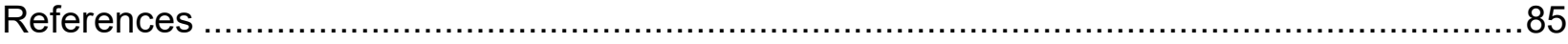




\section{Experimental Procedures}

\section{General Methods}

NMR spectra were recorded on a Bruker Advance III $400 \mathrm{MHz}$, a Bruker Advance HD 400 and an Agilent 400 spectrometer for ${ }^{1} \mathrm{H}(400.13 \mathrm{MHz})$ and ${ }^{13} \mathrm{C}(100.61 \mathrm{MHz}) \mathrm{NMR}$ spectra. A Bruker Ultrashield 600 spectrometer was employed for ${ }^{1} \mathrm{H}(600.13$ $\mathrm{MHz}),{ }^{13} \mathrm{C}(150.90 \mathrm{MHz})$ and ${ }^{15} \mathrm{~N} \mathrm{NMR}(61 \mathrm{MHz})$ spectra. All NMR experiments were performed at $25^{\circ} \mathrm{C}$. Resonances $\delta$ are given in ppm units and referenced to the deuterium signal in the NMR solvents, acetonitrile- $d_{3}\left(\delta_{\mathrm{H}}=1.94 \mathrm{ppm}, \delta_{\mathrm{C}}=1.32,118.26\right.$ ppm). Signal multiplicities are abbreviated as follows: singlet $=\mathrm{s}$, doublet $=\mathrm{d}$, triplet $=\mathrm{t}$, $d q=$ doublet of quartets, multiplet $=\mathrm{m}$.

Normal-structural decomposition (NSD): The NSD method, as developed by Shelnutt and coworkers, ${ }^{[1]}$ was used to delineate, quantify, and illustrate the various distortions modes present in the tetrapyrrole macrocycles. Analysis was performed with the NSD online interface, available at https://www.sengegroup.eu/nsd. ${ }^{[2]}$

Single crystal X-ray crystallography: Crystals were grown following the protocol developed by Hope, liquid-liquid diffusion of $\mathrm{CHCl}_{3}$ and $\mathrm{MeOH}$ with $\mathrm{H}_{2} \mathrm{SO}_{4}{ }^{[3]}$ Diffraction data were collected on a Bruker APEX 2 DUO CCD diffractometer using Incoatec I $\mu \mathrm{S} \mathrm{Cu}-K_{\alpha}(\lambda=1.54178 \AA)$ radiation. Crystal was mounted on a MiTeGen MicroMount and collected at $100(2) \mathrm{K}$ using an Oxford Cryosystems Cobra low-temperature device. Data were collected using omega and phi scans and were corrected for Lorentz and polarization effects using the APEX software suite. ${ }^{[4]}$ Data were corrected for absorption effects using the multi-scan method (SADABS). ${ }^{[5]}$ Using Olex2, the structure was solved with the XT structure solution program, using the intrinsic phasing solution method and refined against $\left|F^{2}\right|$ with $X L$ using least-squares minimization. ${ }^{[6]}$ If electron density was not sufficient, the $C$ and $N$ bound $\mathrm{H}$ atoms were placed in their expected calculated positions and refined using a riding model: $\mathrm{N}-\mathrm{H}=0.88 \AA, \mathrm{C}-\mathrm{H}=0.95-$ $0.98 \AA$, with $U_{\text {iso }}(H)=1.5 U_{\text {eq }}(C)$ for methyl $H$ atoms and $1.2 U_{\text {eq }}(C, N)$. Details of data refinements can be found in Table S4. All images were prepared using Olex2.[6a]

In the structure $\alpha_{2} \beta_{2}-\mathrm{P}\left[\mathrm{SO}_{4}{ }^{2-}\right]\left[\mathrm{HSO}_{4}{ }^{-}\right]_{4}$, two phenyl rings at $\mathrm{C} 5$ and $\mathrm{C} 10$ and one ethyl group are modelled over two locations using DFIX, SIMU, SADI restrains and EADP constraints. In terms of counter anion, only the $\mathrm{SO}_{4}{ }^{2-}$ group is not disordered, while all other $\mathrm{HSO}_{4}{ }^{-}$groups were modelled disordered using rigid groups. Some hydrogen atoms were placed geometrically to compensate for close contacts, the remaining hydrogens could not be located on the disordered $\mathrm{HSO}_{4}{ }^{-}$moieties but were added to the formula to make the formula weight correct. Multiple disordered and partially occupied $\mathrm{H}_{2} \mathrm{O}$ molecules are modelled in the structure using SIMU and ISOR restrains. The weighting scheme was manually adjusted to ensure the goodness of fit was reasonable.

Deposition number 2143572 contain the supplementary crystallographic data for this paper. These data are provided free of charge by the joint Cambridge Crystallographic Data Centre and Fachinformationszentrum Karlsruhe http://www.ccdc.cam.ac.uk/structures.

All calculations were done with Gaussian16 Rev. B.01. ${ }^{[7]}$ A conformational search for $\alpha_{2} \beta_{2}-\mathbf{P} \cdot 10 \mathrm{CSA}(\mathbf{R})$ was performed using a crystal structure of $\alpha_{2} \beta_{2}-\mathrm{P}\left[\mathrm{SO}_{4}{ }^{2-}\right]\left[\mathrm{HSO}_{4}{ }^{-}\right]_{4}$ supramolecules as a starting point for building porphyrin/camphorsulfonic acid supramolecules, followed by optimization in acetonitrile. The geometry optimization and frequencies calculations were performed using BP86 ${ }^{[8]}-\mathrm{D} 3 \mathrm{BJ}{ }^{[9]} /$ def2-SVP ${ }^{[10]}$ - the method which showed a good agreement with the experimental data reported in our previous works. ${ }^{[11]}$ To include acetonitrile effects the $\mathrm{SMD}^{[12]}$ continuum solvent model was used, where molecular surface was represented as Solvent Accessible Surface (SAS) and the Bondi atomic radii were used. A ground state was characterized by absence of imaginary frequencies and more accurate electronic energies were calculated using the BP86D3BJ/def2-TZVPP ${ }^{[10]}$ and SMD model. During conformational search twenty start geometries converged into eight conformers corresponding to the ground state (Table S5) with one major conformer A making up $90 \%$. The geometry were visualized using GaussView 6.1.[13] and is illustrated in figure $\mathrm{S} 14$.

The NMR shielding tensors were calculated at the GIAO[14]-B3LYP ${ }^{[15] / 6-311++G^{* *}[14 a, 16]}$ level of theory using the SMD continuum solvent model to include acetonitrile effects. To calculate the ${ }^{13} \mathrm{C}$ chemical shifts, a scaling factor of $1.0228^{[17]}$ and a reference point TMS (180.7 ppm) were used, for calculation of the ${ }^{15} \mathrm{~N}$ chemical shifts $\mathrm{NH}_{3}$ was used as a reference point (253.70 ppm) (Table S6 - S8). Population analysis was done using the BP86-D3BJ/def2-TZVPP//BP86-D3BJ/def2-SVP level of theory in acetonitrile and $\mathrm{NBO}^{\left[{ }^{18]}\right.}$ approach in acetonitrile. Non-covalent interactions were also analyzed using the SMD (acetonitrile), the BP86-D3BJ/def2-SVP level of theory and AIMAll program version 19.10.12 ${ }^{[19]}$ 


\section{SUPPORTING INFORUATION}

\section{In Text Supplementary Material}

\section{NMR Investigation of the P-10CSA Atropisomers}

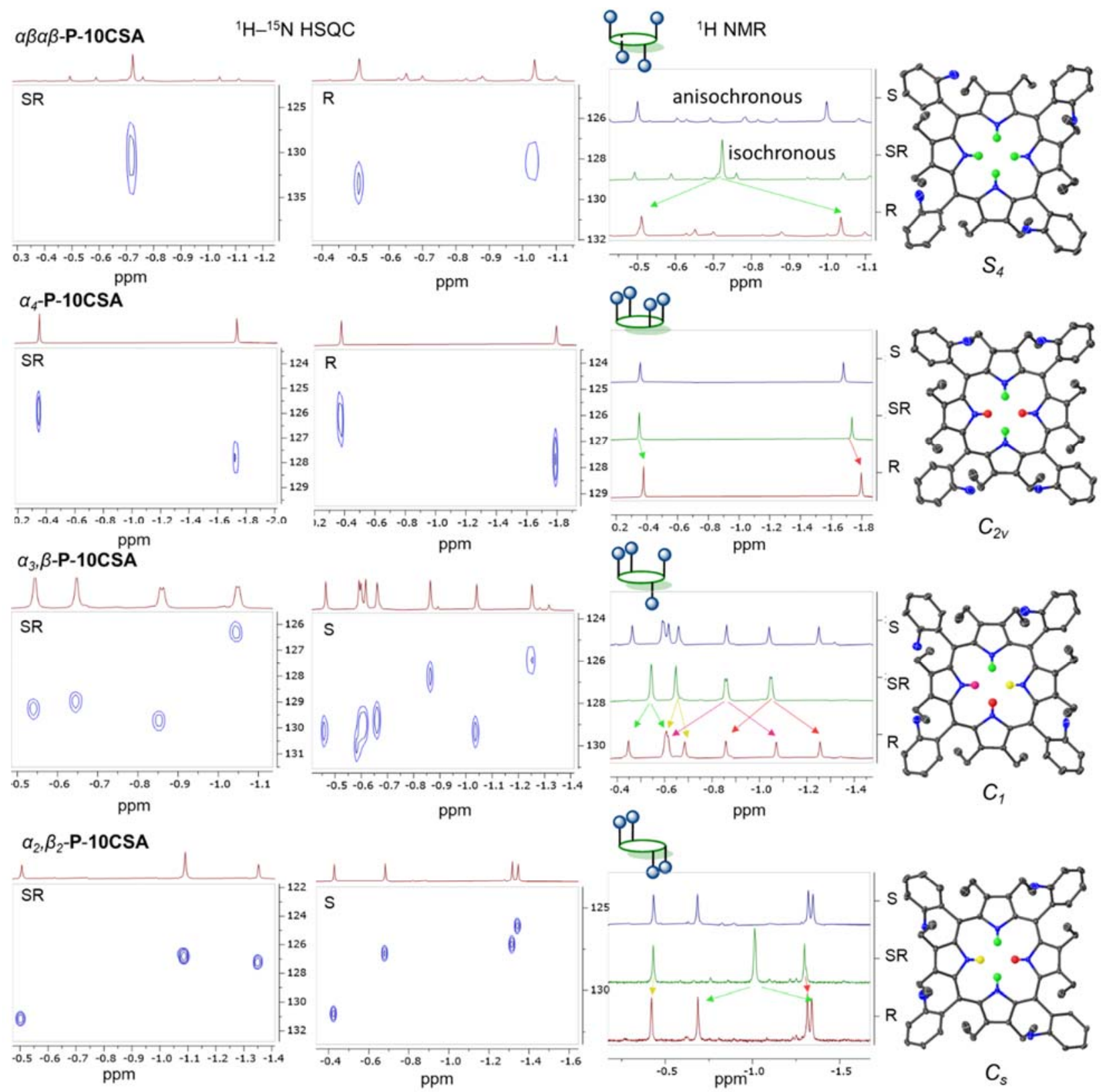

Figure S1. ${ }^{1} \mathrm{H}$ and ${ }^{1} \mathrm{H}-{ }^{15} \mathrm{~N} \mathrm{HSQC} \mathrm{NMR} \mathrm{spectra} \mathrm{obtained} \mathrm{for} 0 \%$ and $100 \%$ e.e. P.10CSA solutions (20 equivalents) in $d_{3}$-acetonitrile. On the right side, a graphical illustration of $\mathbf{P} \cdot 10 \mathrm{CSA}$ atropisomers with corresponding point group notations, inner core system protons are highlighted in different colors correlating to the arrows marked in ${ }^{1} \mathrm{H}$ NMR spectra. 


\section{SUPPORTING INFORVATION}

We performed ${ }^{1} \mathrm{H}$ NMR and ${ }^{1} \mathrm{H}-{ }^{15} \mathrm{~N}$ HSQC analyses of all $\mathbf{P}$ atropisomers with racemic mixtures and enantiopure $10 \mathrm{CSA}(\mathbf{S}$ or $\mathbf{R})$. For the racemic 10CSA solutions, as expected ${ }^{1} \mathrm{H}$ NMR spectra of $\mathbf{P}$ atropisomers remained isochronous (Figure S2). Due to the chiral information transmitted in equal proportions from both chiral components (10CSA R and S), the observed inner core signals resonate in an identical manner to achiral acids (BSA and MSA) previously reported by us. ${ }^{[20]}$ One singlet is observed for $\alpha \beta \alpha \beta-\mathbf{P} \cdot 10 \mathrm{CSA}$, two in $\alpha_{4}$-P.10CSA, three signals of relative intensity $1: 2: 1$ in $\alpha_{2} \beta_{2}$ $\mathbf{P} \cdot 10 \mathrm{CSA}$, and finally the spectrum for the unsymmetrical $\alpha_{3} \beta-\mathbf{P} \cdot 10 \mathrm{CSA}$ atropisomer has four differently shifted signals. The enantiopure solutions are described in the main text.
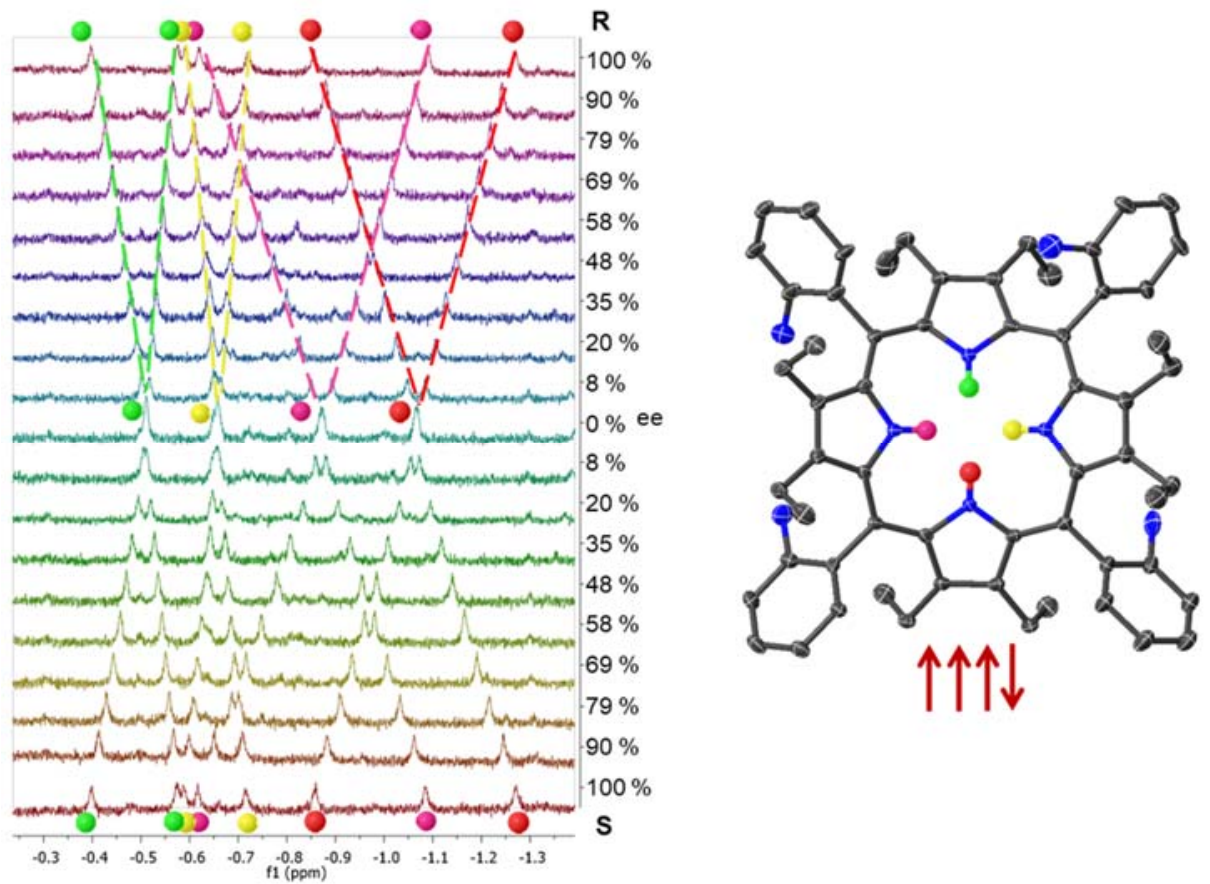

Figure S2. ${ }^{1} \mathrm{H}$ NMR titration studies of the $\alpha_{3} \beta$-P inner core system using different ratios of chiral components (10CSA R and S, 20 eq.). Spectra recorded in $\mathrm{CD}_{3} \mathrm{CN}$

\section{CSA Additions to $\alpha_{2} \beta_{2}-P$}

The gradual addition of $10 \mathrm{CSA}(\mathbf{R})$ into the $\mathrm{CD}_{3} \mathrm{CN}$ solution of $\alpha_{2} \beta_{2}-\mathrm{P}$ resulted in downfield shifts of the ${ }^{1} \mathrm{H}$ NMR aromatic region signals with the and observable splitting pattern of the o-ArH (Figure S3), while the split pyrrolic $\mathrm{N}-\mathrm{H}$ were upfield shifted upon emerging at $\sim 3$ eq. of $10 \mathrm{CSA}(\mathbf{R})$ in the ${ }^{1} \mathrm{H}$ NMR spectra (Figures S4 and S5). The magnitude of chemical shifts of split peaks $(\Delta \sigma)$ drastically increased over the first $\sim 7$ eq., while the later additions resulted in marginal changes to $\Delta \sigma$ (Figure S6). 


\section{SUPPORTING INFORMATION}

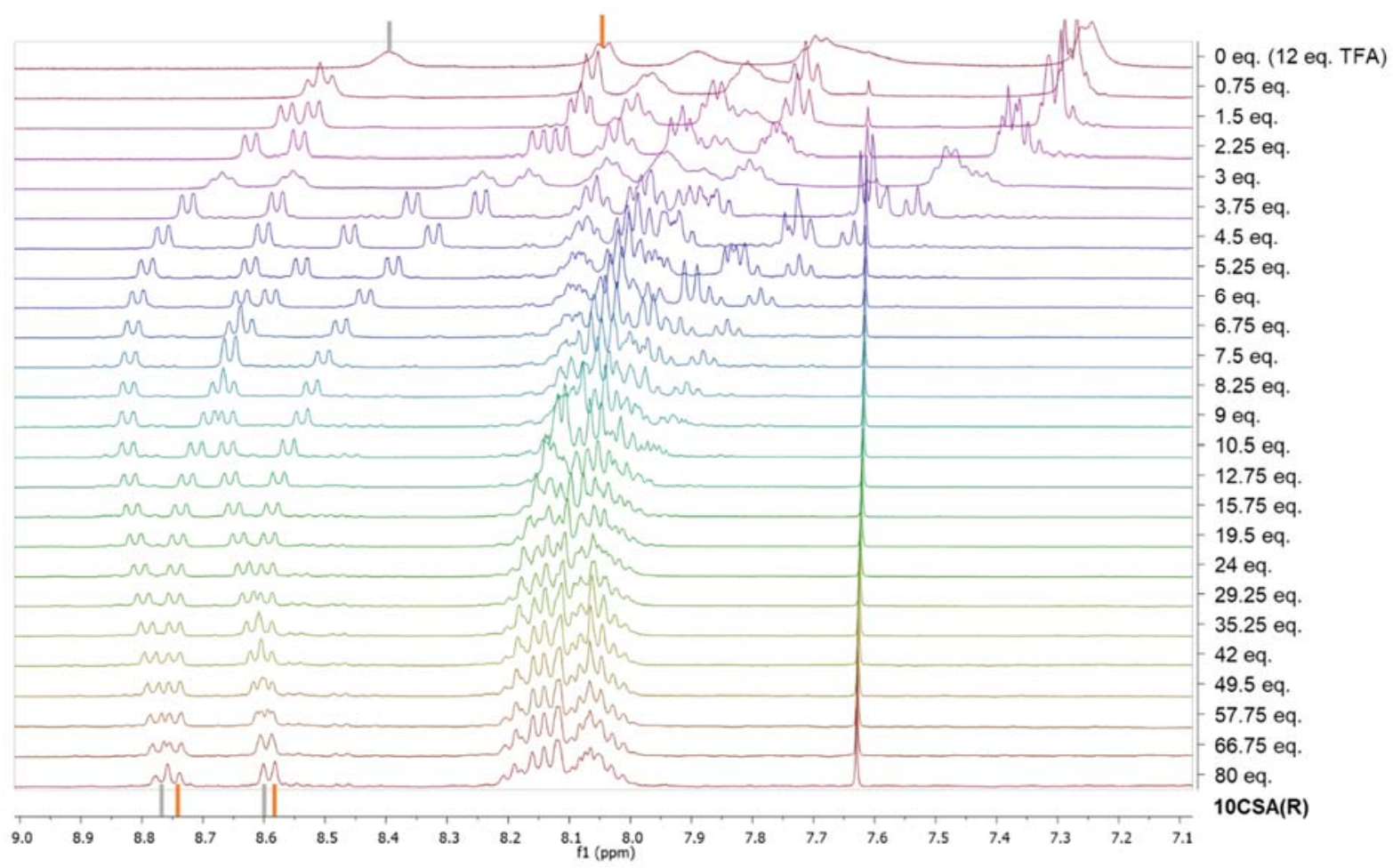

Figure S3. ${ }^{1} \mathrm{H}$ NMR of the $\alpha_{2} \beta_{2}-\mathrm{P}$ aromatic region dependence to the $10 \mathrm{CSA}(\mathrm{R})$ equivalents, recorded in $\mathrm{CD}_{3} \mathrm{CN}$. The addition of 12 eq. of TFA was to solubilize and protonate $\alpha_{2} \beta_{2}-\mathbf{P}$. Highlighted in grey and orange is two different splitting $\mathrm{O}-\mathrm{Ar}-\mathrm{H}$ regions.

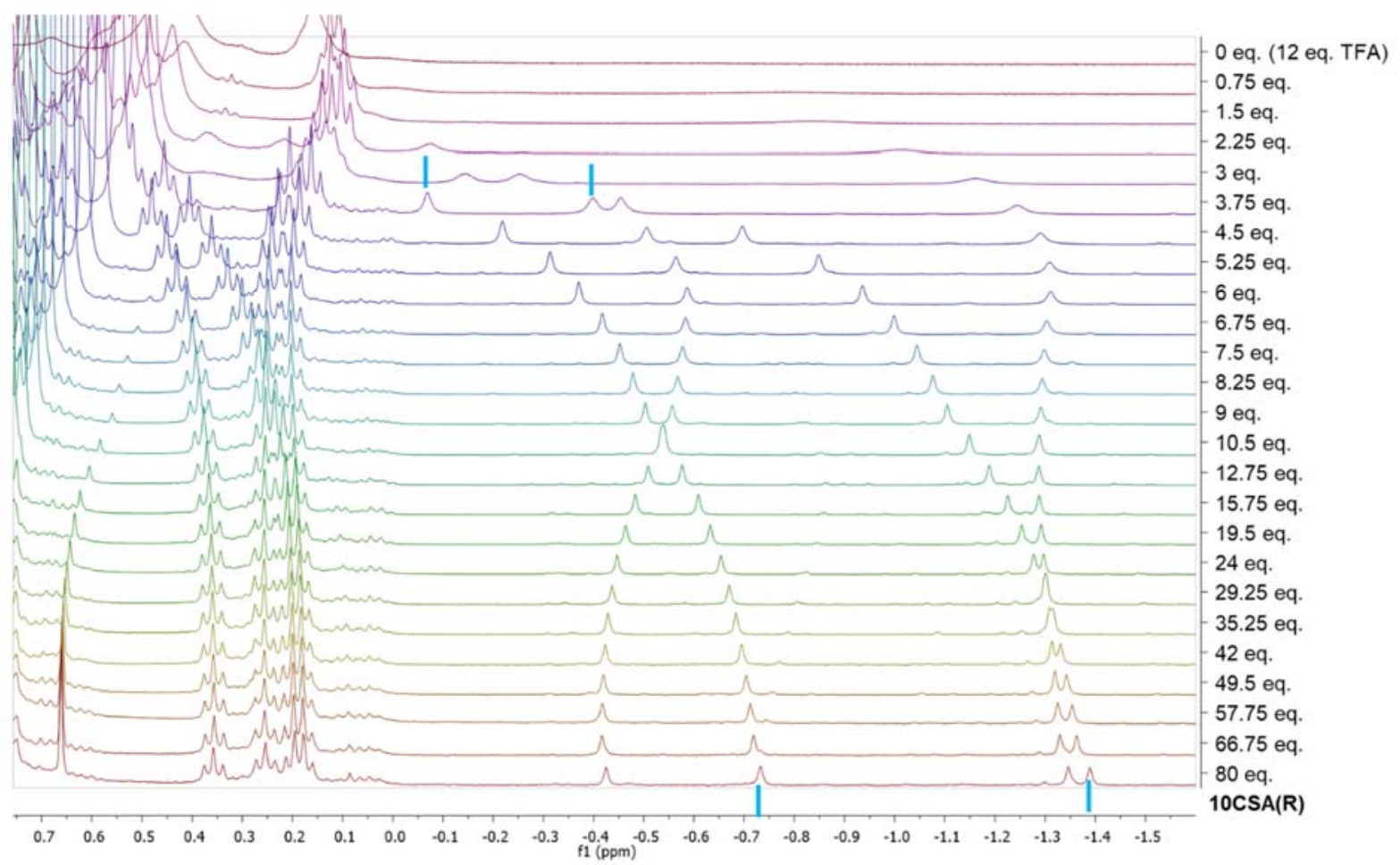

Figure S4. ${ }^{1} \mathrm{H}$ NMR of the $\alpha_{2} \beta_{2}-\mathrm{P} \mathrm{N}-\mathrm{H}$ and $\mathrm{CH}_{3}$ regions dependence to the $10 \mathrm{CSA}(\mathbf{R})$ equivalents, recorded in $\mathrm{CD}_{3} \mathrm{CN}$. The addition of 12 eq. of TFA was to solubilize and protonate $\alpha_{2} \beta_{2}-\mathbf{P}$. Highlighted in blue are two different splitting $\mathrm{N}-H$ resonance signals. 


\section{SUPPORTING INFORMATION}

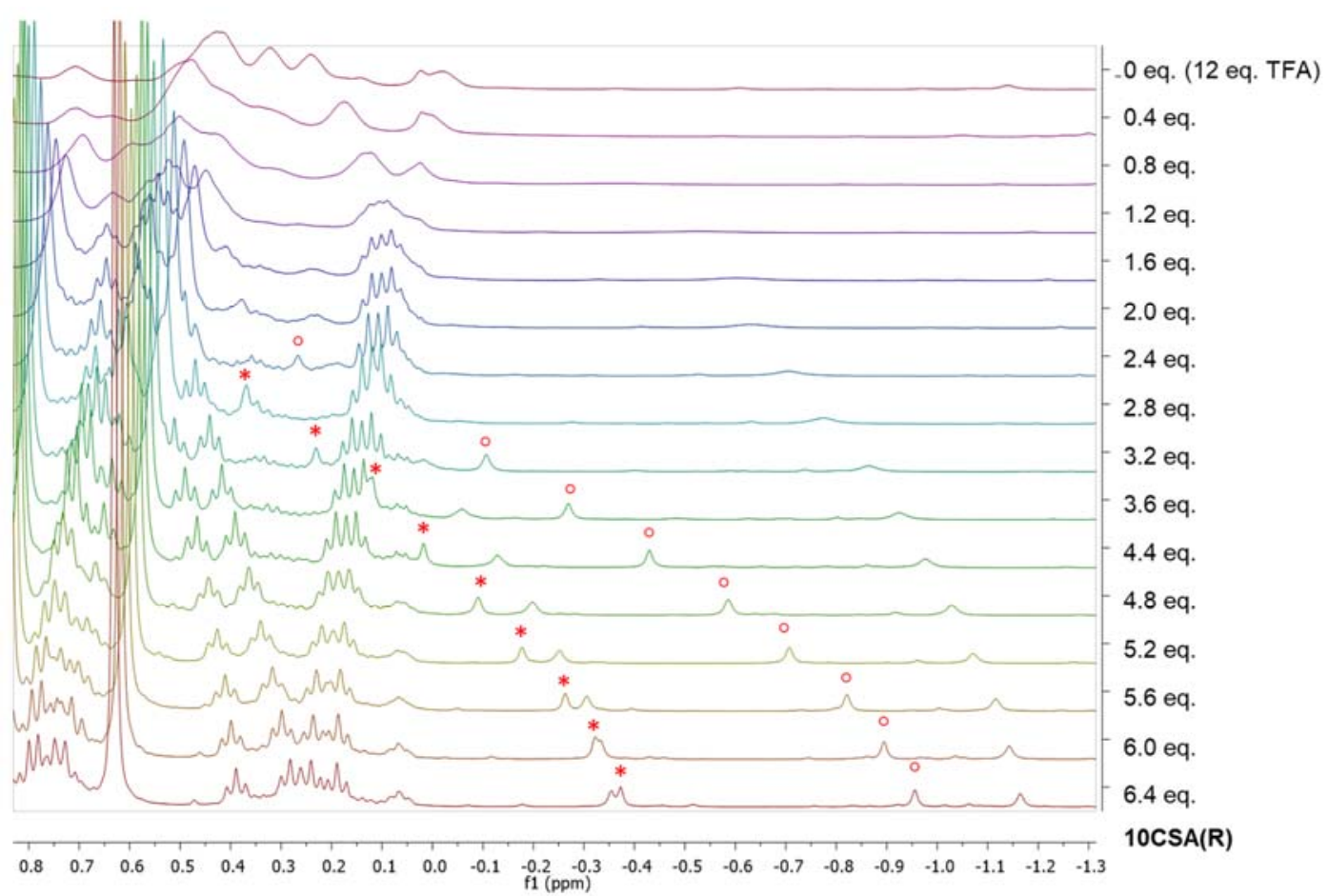

Figure S5. ${ }^{1} \mathrm{H}$ NMR of the $\alpha_{2} \beta_{2}-\mathrm{P} \mathrm{N}-H$ regions dependence to the $10 \mathrm{CSA}(\mathbf{R})$ equivalents, recorded in $\mathrm{CD}_{3} \mathrm{CN}$. The addition of 12 eq. of TFA was to solubilize and protonate $\alpha_{2} \beta_{2}$-P. Highlighted with red dot and star is two different splitting $\mathrm{N}-H$ resonance signals. Note, these spectra were recorded with higher concentrations of $\alpha_{2} \beta_{2}-\mathrm{P}$ expecting to get a better resolution of the inner $\mathrm{N}-\mathrm{H}$. From the obtained spectra it appears that the inner core system of interest originates downfield shifted and overlays with the aliphatic $\mathrm{CH}_{3}$ region.
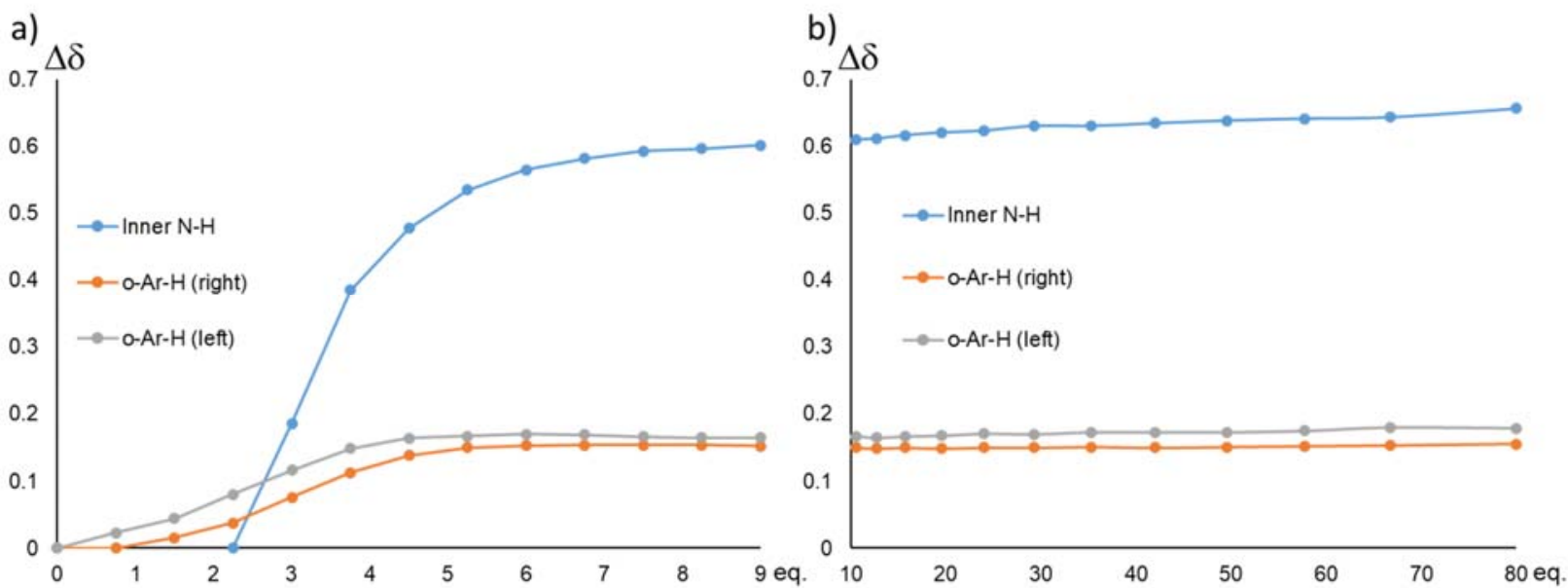

Figure S6. A graph showing the magnitude of the split chemical shifts $\Delta \delta$ in the inner $\mathrm{N}-H$ and o-Ar-H of the phenyl rings independence on the 10CSA(R) equivalents, a) 0 to 9 eq., b) 10 to 80 eq. Recorded in $\mathrm{CD}_{3} \mathrm{CN}$ with the addition of 12 eq. of TFA to solubilize and protonate $\alpha_{2} \beta_{2}$-P. The color coding corresponds to the highlighted signals in Figures S3 and S4. 


\section{SUPPORTING INFORVATION \\ Influence of Water on the Complexation}

We have previously mentioned that one of the best-known pro-CSA ( $\left.\mathbf{B z}_{2} \mathbf{O} \mathbf{x P}\right)$ suffers serious sensitivity issues due in part to $\mathrm{N}$-alkylation. ${ }^{[21]}$ Additionally, the competitive binding of water molecules significantly contributes to the magnitude of splitting $\Delta \sigma$. Trace amounts of water in solutions or titrants results in the necessary use of high guest concentrations to obtain well-resolved spectra. The hypersensitivity towards water is a major limitation for functional pro-CSA, since water is ubiquitous, avoiding it is at least tedious if not almost impossible for most of the solvents and reagents, especially for the analytes bearing high polarity. Hence, investigation of water influence as a competitive agent to the enantio-pure $\alpha_{2} \beta_{2}-\mathbf{P} \cdot 10 \mathrm{CSA}(\mathbf{S})$ complex in $\mathrm{CD} 3 \mathrm{CN}$ was carried out. From the first instance, the gradual addition of water has a considerable effect on $\Delta \sigma$. By the addition of 240 eq. of water, the $\Delta \sigma$ of $\mathrm{N}-\mathrm{H}$ has contracted by $0.132 \mathrm{ppm}$. (Figure S9). Despite the competitive nature of water, the overall binding strength of $10 \mathrm{CSA}(\mathrm{S})$ remains observable considering the $\sim 12.5$ fold higher water consistency in the solution. Even at substantially higher (>4000 eq.) quantity of water the signals remained anisochronous highlighting the strong relationship between the host and chiral guest (Figure S8-S9).
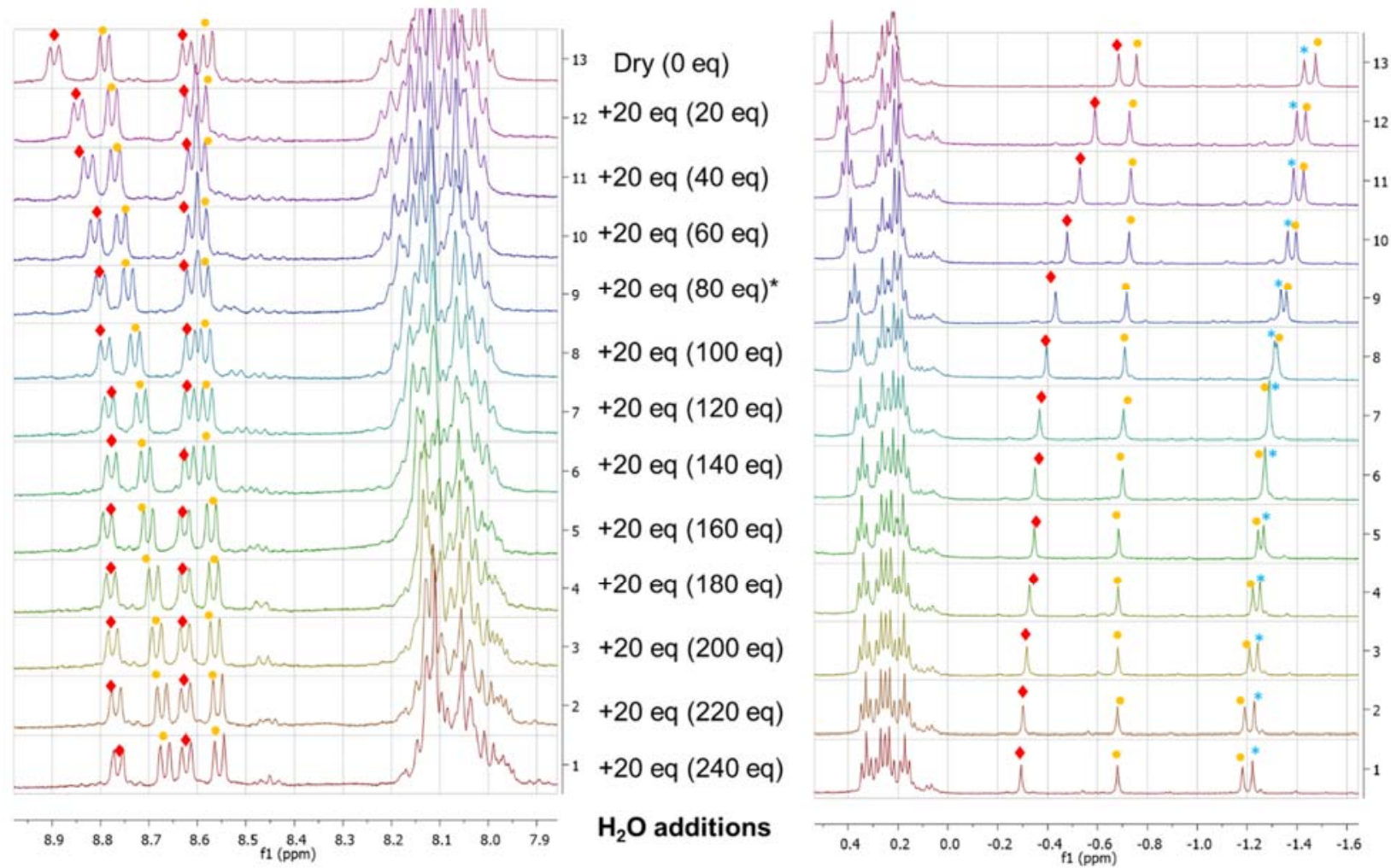

Figure S7. ${ }^{1} \mathrm{H}$ NMR titration with $\mathrm{H}_{2} \mathrm{O}\left(0-240\right.$ eq) of $\alpha_{2} \beta_{2}-\mathbf{P} \cdot 10 \mathrm{CSA}(\mathbf{S})(19 \mathrm{eq})$. The split inner $\mathrm{N}-\mathrm{H}$ is highlighted with orange dots. In the aromatic region, the split $\mathrm{o}-\mathrm{ArH}$ signals are highlighted with orange and red dots. Spectra recorded in $\mathrm{CD}_{3} \mathrm{CN}$ 


\section{SUPPORTING INFORIMATION}
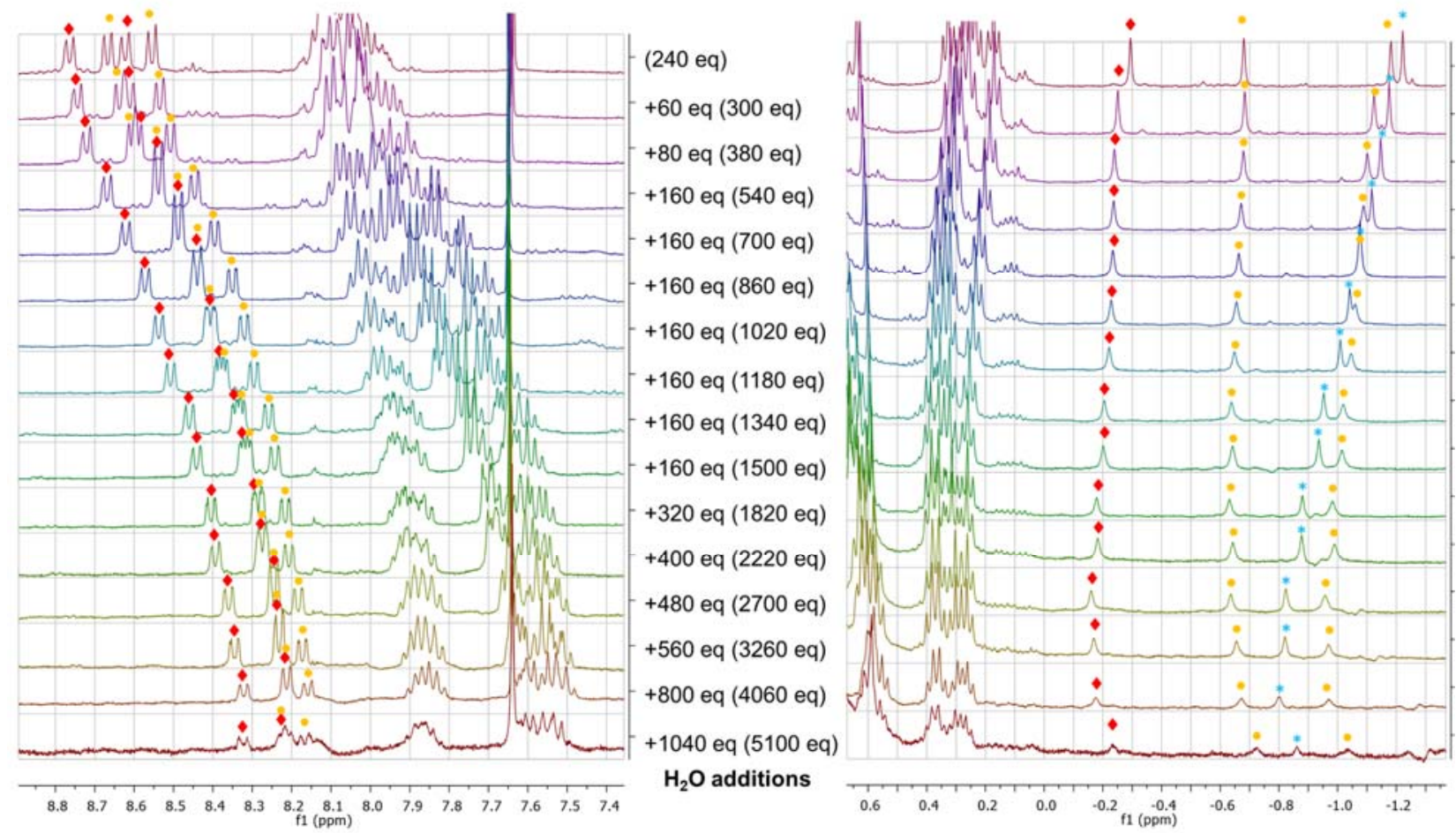

Figure S8. ${ }^{1} \mathrm{H}$ NMR titration with $\mathrm{H}_{2} \mathrm{O}\left(240-5100\right.$ eq) of $\alpha_{2} \beta_{2}$-P·10CSA(S) (19 eq). The split inner $\mathrm{N}-H$ is highlighted with orange dots. In the aromatic region, the split $\mathrm{o}-\mathrm{ArH}$ signals are highlighted with orange and red dots. Spectra recorded in $\mathrm{CD}_{3} \mathrm{CN}$.
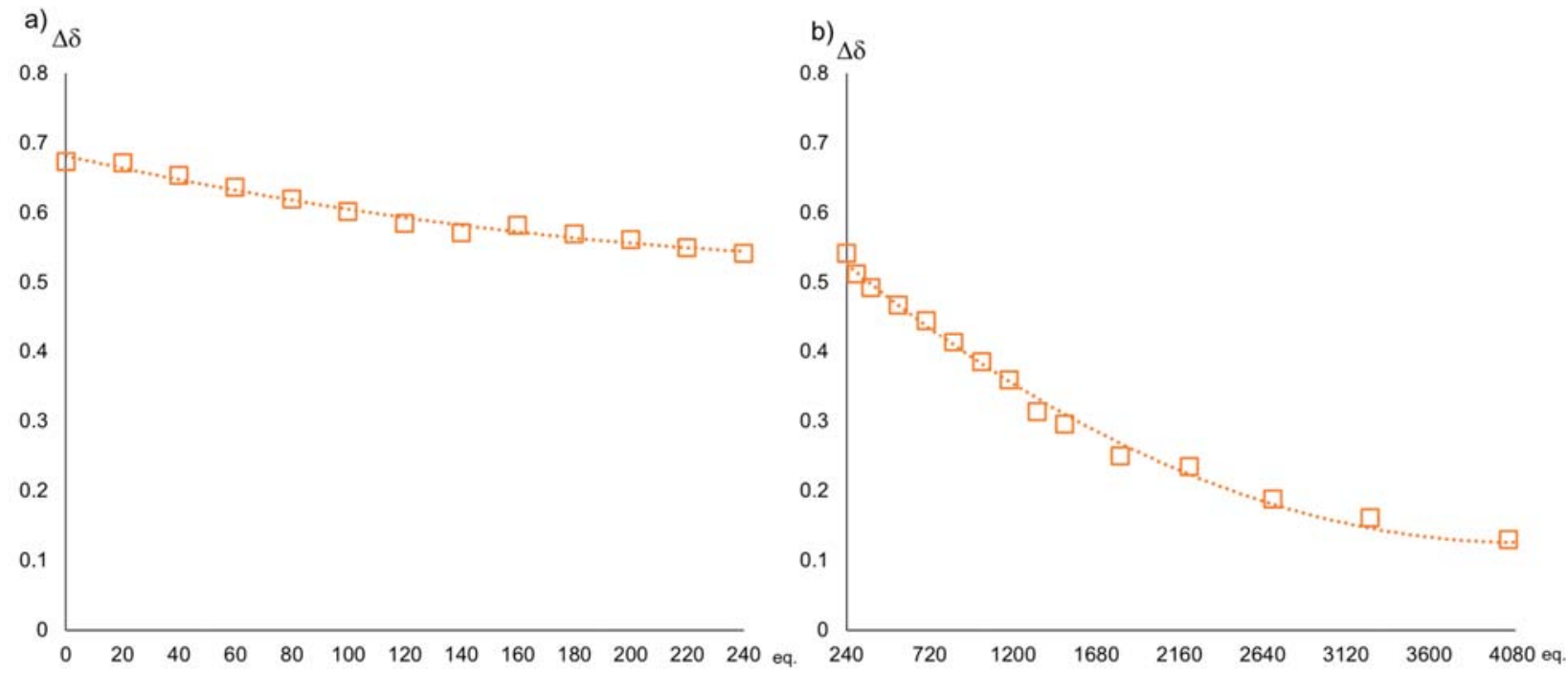

Figure S9. The graph of $\alpha_{2} \beta_{2}-\mathbf{P} \cdot 10 \mathrm{CSA}(\mathbf{S})$ (19 eq) representing $\mathrm{N}-H \Delta \sigma$ dependence to the various additions of water a) 0-240 eq. b) 240-5100 eq. 


\section{SUPPORTING INFORMATION \\ Detection of Enantiomeric Excess}

For example, 19 eq. of an unknown enantiomeric mixture of 10CSA was complexed with $\alpha_{2} \beta_{2}$-P (Figure S11). The inner core system shows clearly split resonance signals with $\Delta \sigma=0.13 \mathrm{ppm}$. A small addition of $10 \mathrm{CSA}(\mathbf{R})$ decreased $\Delta \sigma(0.051 \mathrm{ppm})$, while a similar amount of 10CSA(S) increased $\Delta \sigma(0.224 \mathrm{ppm})$ revealing the predominant enantiomeric identity $(S>R)$. Next, a linear calibration plot with 7 data points (e.e.10csa(s) $=0,48,58,69,79,90,100 \%$ ) was constructed. It is worth noting that it is possible to generate a calibration curve from a single measured enantiopure point due to the linear dependency with the second point being $\Delta \sigma=0 \mathrm{ppm}$. where e.e. $=0 \%$. Lastly, the $\Delta \sigma$ can be fitted to the calibration plot and reveal the unknown e.e. (21\%) based on the inner $\mathrm{N}-\mathrm{H}$ chemical shift difference between the split peaks (for more information on this example see figure S12). The same principle can be applied to quickly test the purity of enantiomers, i.e. by equally pre-mixing stereoisomers of interest with opposite chirality, in the event of matching purity, the $\alpha_{2} \beta_{2}-\mathbf{P}$ signals will remain isochronous. Overall, monitoring changes of this inner core system in a model chiral environment demonstrates a powerful tool for easy and sensitive detection of enantiomeric compositions.

a) $\mathrm{O}-\mathrm{ArH}$

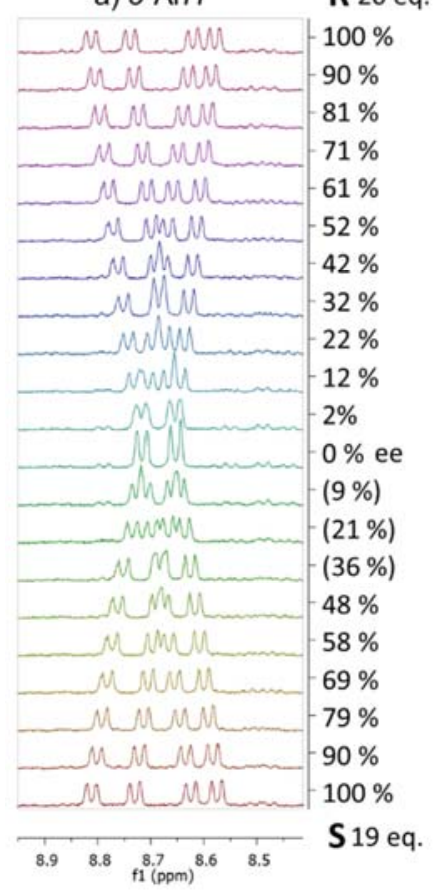

b) $\mathrm{CH}_{3}$

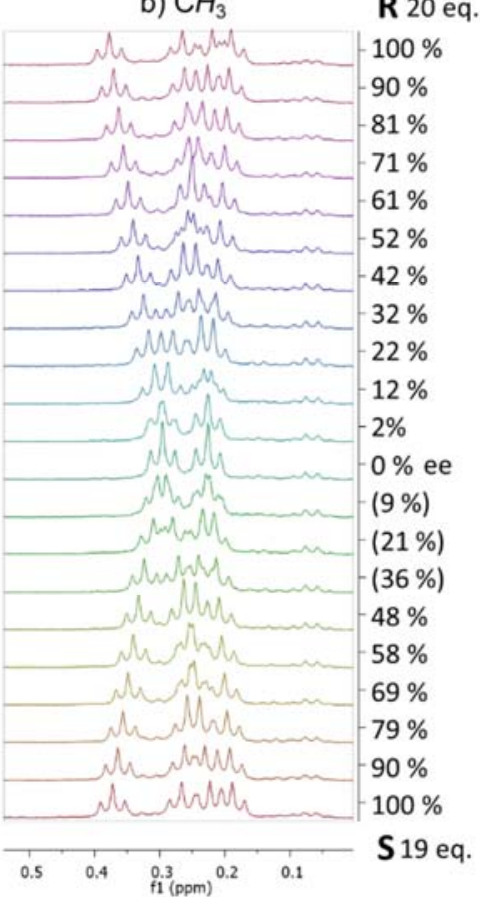

c) $\mathrm{NH}$

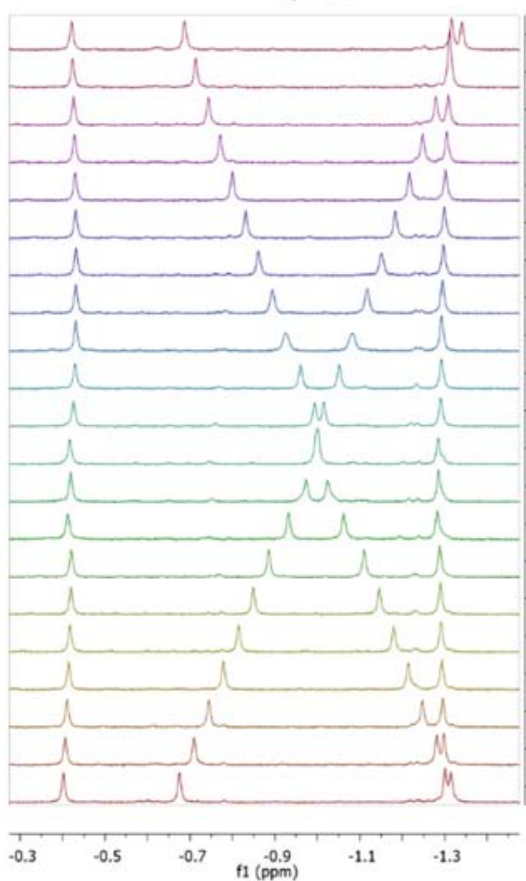

R 20 eq. $100 \%$ $90 \%$ $81 \%$ $71 \%$ $61 \%$ $52 \%$ $42 \%$ $32 \%$ $22 \%$ $12 \%$ $2 \%$ $0 \%$ ee (9\%) $(21 \%)$ $(36 \%)$ $48 \%$ $58 \%$ $69 \%$ $79 \%$ $90 \%$ $100 \%$ S 19 eq.

Figure S10. ${ }^{1} \mathrm{H}$ NMR representation of the three non-overlapping regions for the detection of e.e. (values in brackets calculated from the plot see Figure S8-S9: a) o-ArH, g) $\mathrm{CH}_{3}$, and $\mathrm{h}$ ) inner core system $\mathrm{N}-\mathrm{H}$. Two different concentrations of analytes ( 20 eq. of $10 \mathrm{CSA}(\mathbf{R})$ and 19 eq. of $10 \mathrm{CSA}(\mathbf{S})$ ) were used to highlight the diversity of the enantiomeric excess detection using $\alpha_{2} \beta_{2}-\mathrm{P}$. Spectra recorded in $\mathrm{CD}_{3} \mathrm{CN}$. 


\section{SUPPORTING INFORMATION}

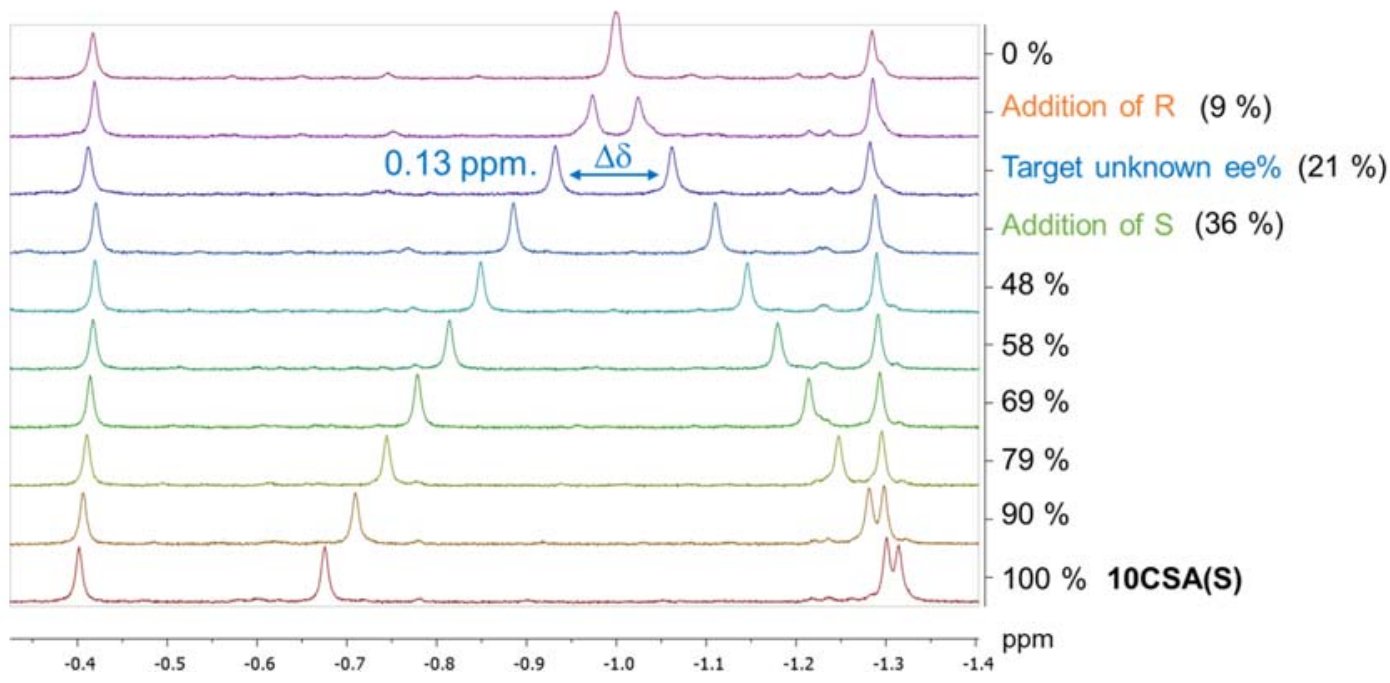

Figure S11. The ${ }^{1} \mathrm{H}$ NMR spectra of the inner core system $\mathrm{N}-\mathrm{H}$ unknown e.e.\% target compound (highlighted in blue), after small 10CSA(R) (red) and 10CSA(S) (green) additions, and the rest of the spectra for the construction of the calibration curve. Spectra recorded in $\mathrm{CD}_{3} \mathrm{CN}$.

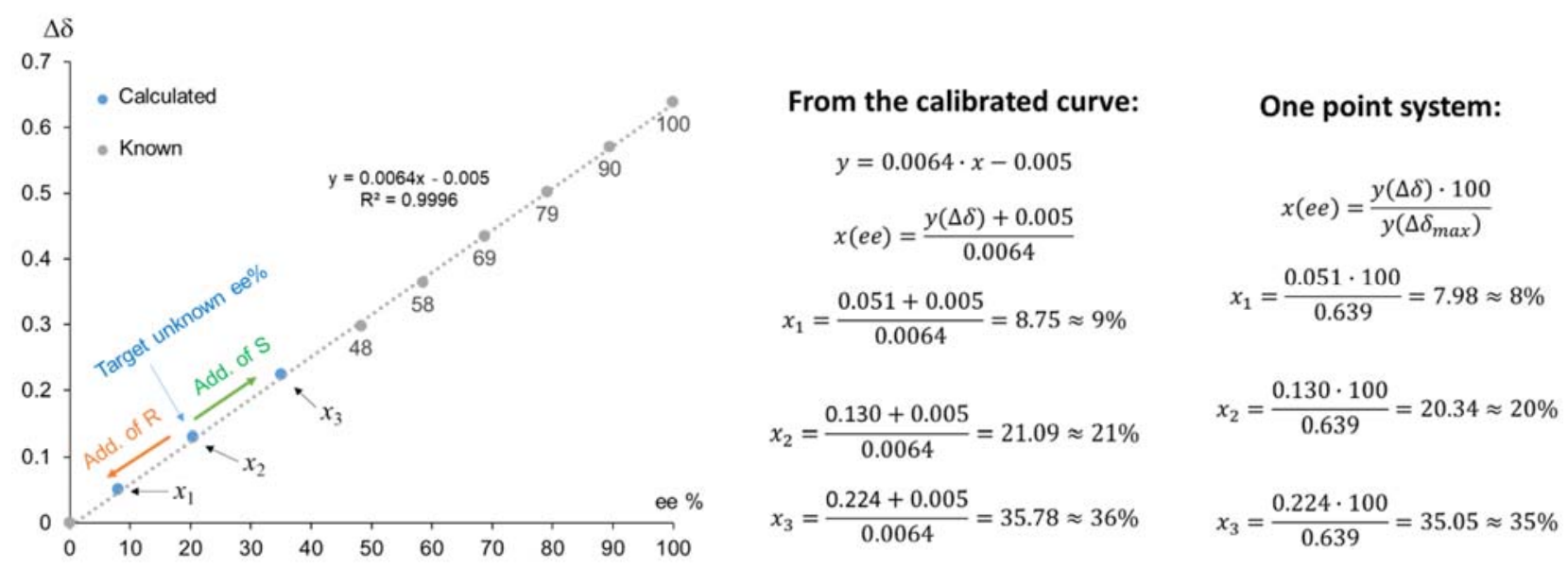

Figure S12. Graph of the $\Delta \sigma$ dependence on the ee\% with 19eq. of 10CSA. Measured from the ${ }^{1} \mathrm{H}$ NMR split $\mathrm{N}-$ $H$ signals recorded in $\mathrm{CD}_{3} \mathrm{CN}$ (at 100 ee\% -19 eq. $\alpha_{2} \beta_{2}$-P.10CSA(S)). On the right side: calculations of the unknown ee from the calibrated curve and using a one-point system. Note, calculations using a one-point system should only be used for quick, approximate determinations of the ee. 
To further understand the transfer of chirality to the atropisomeric receptor systems $\mathbf{P}$, we have performed 2D NMR analyses with enantiopure 10CSA. The ${ }^{15} \mathrm{~N}$ resonance signals obtained from ${ }^{1} \mathrm{H}-{ }^{15} \mathrm{~N}$ HSQC varied from 125 to $135 \mathrm{ppm}$ and correlated well with the corresponding inner core system protons. The $\Delta \sigma_{\max }$ of the ${ }^{15} \mathrm{~N}$ were found to be $\alpha_{2} \beta_{2}-\mathbf{P}(1.67 \mathrm{ppm})>\alpha \beta \alpha \beta-\mathbf{P}(1.03 \mathrm{ppm})>\alpha_{3} \beta-\mathbf{P}(0.77 \mathrm{ppm})>\alpha_{4^{-}}$ $\mathbf{P}(0 \mathrm{ppm})$ (Table S2). Full 2D NMR analyses to determine all of the resonance signals were done for $\alpha_{2} \beta_{2}-\mathbf{P}, \alpha \beta \alpha \beta-\mathbf{P}$ and $\alpha_{4}-\mathbf{P}$ with 10CSA(S). Unfortunately, due to formation of diastereomers in $\alpha_{3} \beta-$ $\mathbf{P} \cdot 10 \mathrm{CSA}(\mathrm{S})$ the large number of signals hampered detailed NMR analysis (Figure S59-S64). While some of the ${ }^{1} \mathrm{H}$ resonance signals in $\alpha_{4}-\mathbf{P} \cdot 10 \mathrm{CSA}(\mathbf{S})$ showed possible splitting patterns, the $\Delta \sigma_{\max }$ were found to not surpass $0.08 \mathrm{ppm}$. In $\alpha_{2} \beta_{2}-\mathbf{P} \cdot 10 \mathrm{CSA}(\mathbf{S})$ and $\alpha \beta \alpha \beta-\mathbf{P} \cdot 10 \mathrm{CSA}(\mathbf{S})$ other than inner core $\mathrm{N}-H$ the ${ }^{1} \mathrm{H} \Delta \sigma_{\max }$ showed to be highest in o-Ar- $\mathrm{H}(0.16-0.2 \mathrm{ppm})$ and certain $\mathrm{CH}_{3}$ groups $(0.22 \mathrm{ppm}$ in $\alpha \beta \alpha \beta-$ $\mathbf{P} 2^{2}$ and $12^{2}, 7^{2}$ and $17^{2} ; 0.16 \mathrm{ppm}$ in $\alpha_{2} \beta_{2}-\mathbf{P} 2^{2}$ and $13^{2}$ positions). Only particular ${ }^{13} \mathrm{C}$ in the $\alpha \beta \alpha \beta-$ $\mathbf{P} \cdot 10 \mathrm{CSA}(\mathbf{S})$ 24-atom macrocycle displayed $\Delta \sigma_{\max }$ with $>0.11 \mathrm{ppm}(0.47 \mathrm{ppm}$ between 6 and 16,1 and 11; 0.8 ppm between 8 and 18, 3 and 13 positions). Similarly, in $\alpha_{4}-\mathbf{P} \cdot 10 \mathrm{CSA}(\mathbf{S})$ only between 1 and 4 , 13 and 12 positions the ${ }^{13} \mathrm{C} \Delta \sigma_{\max }(0.23 \mathrm{ppm})$ was observed to be $>0.11 \mathrm{ppm}$. On the other hand, the majority of the $\alpha_{2} \beta_{2}-\mathbf{P} \cdot 10 \mathrm{CSA}(\mathbf{S})$ pyrrolic ${ }^{13} \mathrm{C}$ displayed $\Delta \sigma_{\max }>0.2 \mathrm{ppm}$, reaching $0.98 \mathrm{ppm}$ between 1 and 14 positions. In the phenyl rings, except for the previously discussed significant ${ }^{13} \mathrm{C} \Delta \sigma_{\max }(1.3 \mathrm{ppm})$ between $15^{6}$ and $20^{6}$ in $\alpha_{2} \beta_{2}$-P.10CSA(S), other phenyl positions and likewise in other atropisomeric species displayed $\Delta \sigma_{\max }<0.31 \mathrm{ppm}$. 


\section{SUPPORTING INEORMATION}

Table S1. Comparison of ${ }^{13} \mathrm{C}$ and ${ }^{15} \mathrm{~N}$ atom resonances $\alpha_{2} \beta_{2}$-P.BSA, ${ }^{[20]} \alpha_{2} \beta_{2}-\mathbf{P} \cdot 10 \mathrm{CSA}(\mathbf{S})$ and $\alpha_{2} \beta_{2}-\mathbf{P} \cdot \mathbf{1 0 C S A}(\mathbf{S R})$ complexes. The $\Delta \sigma_{\max }(\mathrm{ppm})$ in ${ }^{13} \mathrm{C}$ and ${ }^{15} \mathrm{~N}$ atoms of $\alpha_{2} \beta_{2}-\mathbf{P} \cdot \mathbf{1 0 C S A}(\mathbf{S})$ recorded with 20 eq. of analyte. The signal positions were determined using various NMR techniques $\left(\mathrm{CD}_{3} \mathrm{CN}\right.$ ). Top left side, illustration of the $\alpha_{2} \beta_{2}-\mathbf{P}$ (blue above and red - below the plane) with corresponding positions. The highlighted positions in the illustration on the top right side shows $\Delta \sigma \geq 0.3 \mathrm{ppm}$ (atoms in blue are peripheral nitrogen atoms that did not resonate).<smiles>CCC1=C(CC)C(=C(c2ccccn2)c2[nH]c(C(c3ccccc3)c3ccccc3)c(CC)c2CC)N=C1c1[nH]c(C(=C2CCCCC2)c2ccccc2)c(CC)c1CC</smiles>

\begin{tabular}{|c|c|c|c|}
\hline Pos. & BSA & $\begin{array}{l}\text { CSA-SR } \\
\overline{O C} \text { or } \bar{O} N\end{array}$ & CSA-S \\
\hline 1 & 144.1 & 144.1 & 143.7 \\
\hline 2 & 142.0 & 142.1 & 142.3 \\
\hline $2^{1}$ & 19.1 & 19.3 & 19.5 \\
\hline $2^{2}$ & 15.9 & 16.0 & 16.0 \\
\hline 3 & 143.9 & 143.4 & 143.2 \\
\hline $3^{1}$ & 20.5 & 20.6 & 20.5 \\
\hline $3^{2}$ & 15.2 & 15.6 & 15.5 \\
\hline 4 & 143.9 & 144.3 & 144.5 \\
\hline 5 & 114.9 & 114.1 & 113.9 \\
\hline $5^{1}$ & 132.1 & 132.0 & 132.0 \\
\hline $5^{2}$ & 133.1 & 133.1 & 133.1 \\
\hline $5^{3}$ & 130.6 & 130.5 & 130.6 \\
\hline $5^{4}$ & 134.2 & 133.8 & 133.8 \\
\hline $5^{5}$ & 126.4 & 125.9 & 125.9 \\
\hline $5^{6}$ & 139.8 & 141.0 & 141.1 \\
\hline 6 & 142.8 & 143.0 & 142.8 \\
\hline 7 & 142.9 & 141.9 & 141.4 \\
\hline $7^{1}$ & 19.1 & 19.4 & 19.2 \\
\hline $7^{2}$ & 15.9 & 16.4 & 16.3 \\
\hline 8 & 142.9 & 141.9 & 142.3 \\
\hline $8^{1}$ & 19.1 & 19.4 & 19.4 \\
\hline $8^{2}$ & 15.9 & 16.4 & 16.3 \\
\hline 9 & 142.8 & 143.0 & 143.1 \\
\hline 10 & 114.9 & 114.1 & 114.3 \\
\hline $10^{1}$ & 132.1 & 132.0 & 131.9 \\
\hline $10^{2}$ & 133.1 & 133.1 & 133.2 \\
\hline $10^{3}$ & 130.6 & 130.5 & 130.4 \\
\hline $10^{4}$ & 134.2 & 133.8 & 133.8 \\
\hline $10^{5}$ & 126.4 & 125.9 & 125.9 \\
\hline $10^{6}$ & 139.8 & 141.0 & 141.1 \\
\hline 11 & 143.9 & 144.3 & 144.1 \\
\hline 12 & 143.9 & 143.4 & 143.4 \\
\hline
\end{tabular}
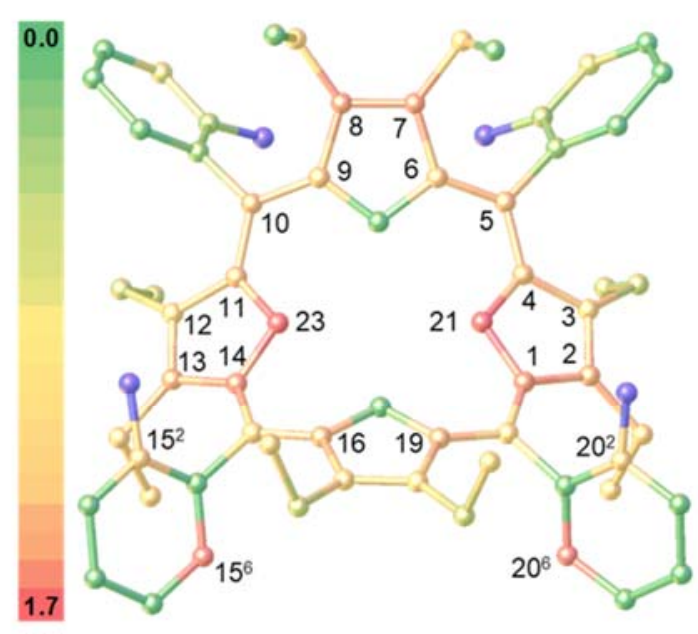

$\Delta \delta$

BSA

CSA-SR CSA-S Ō $\mathrm{C}$ or $\bar{O} \mathrm{~N}$

$\begin{array}{llll}12^{1} & 20.5 & 20.6 & 20.6\end{array}$

$\begin{array}{llll}12^{2} & 15.2 & 15.6 & 15.6\end{array}$

$\begin{array}{llll}13 & 142.0 & 142.1 & 141.7\end{array}$

$\begin{array}{llll}13^{1} & 19.1 & 19.3 & 19.3\end{array}$

$\begin{array}{llll}13^{2} & 15.9 & 16.0 & 15.8\end{array}$

$\begin{array}{llll}14 & 144.1 & 144.1 & 144.7\end{array}$

$\begin{array}{llll}15 & 112.0 & 112.6 & 112.5\end{array}$

$\begin{array}{llll}15^{1} & 132.0 & 132.3 & 132.3\end{array}$

$\begin{array}{llll}15^{2} & 133.0 & 134.0 & 134.3\end{array}$

$\begin{array}{llll}15^{3} & 130.5 & 129.9 & 129.7\end{array}$

$\begin{array}{llll}15^{4} & 134.2 & 133.8 & 133.8\end{array}$

$\begin{array}{llll}15^{5} & 125.9 & 126.3 & 126.1\end{array}$

$\begin{array}{llll}15^{6} & 140.4 & 139.3 & 139.9\end{array}$

$\begin{array}{llll}16 & 144.9 & 145.6 & 145.9\end{array}$

$\begin{array}{llll}17 & 145.6 & 144.4 & 144.4\end{array}$

$\begin{array}{llll}17^{1} & 20.8 & 20.6 & 20.6\end{array}$

$\begin{array}{llll}17^{2} & 15.4 & 15.4 & 15.3\end{array}$

$\begin{array}{llll}18 & 145.6 & 144.4 & 144.2\end{array}$

$\begin{array}{llll}18^{1} & 20.8 & 20.6 & 20.5\end{array}$

$\begin{array}{llll}18^{2} & 15.4 & 15.4 & 15.5\end{array}$

$\begin{array}{llll}19 & 144.9 & 145.6 & 145.3\end{array}$

$\begin{array}{llll}20 & 112.0 & 112.6 & 112.7\end{array}$

$\begin{array}{llll}20^{1} & 132.0 & 132.3 & 132.3\end{array}$

$\begin{array}{llll}20^{2} & 133.0 & 134.0 & 134.6\end{array}$

$\begin{array}{llll}20^{3} & 130.5 & 129.9 & 129.7\end{array}$

$\begin{array}{llll}20^{4} & 134.2 & 133.8 & 133.8\end{array}$

$\begin{array}{llll}20^{5} & 125.9 & 126.3 & 126.1\end{array}$

$\begin{array}{llll}20^{6} & 140.4 & 139.3 & 138.6\end{array}$

$\begin{array}{lll}21 & 127.0 & 127.6\end{array}$

128.5

128.0

$\begin{array}{lll}127.0 & 127.6 & 126.9\end{array}$

$125.9 \quad 131.9$

132.2

\begin{tabular}{|c|c|}
\hline \multicolumn{2}{|c|}{$\overline{\delta C}$ or $\overline{~ N}$} \\
\hline Pos. & $\Delta \delta$ \\
\hline 1 and 14 & 0.98 \\
\hline 2 and 13 & 0.59 \\
\hline $2^{1}$ and $13^{1}$ & 0.26 \\
\hline $2^{2}$ and $13^{2}$ & 0.22 \\
\hline 3 and 12 & 0.22 \\
\hline $3^{1}$ and $12^{1}$ & 0.12 \\
\hline $3^{2}$ and $12^{2}$ & 0.12 \\
\hline 4 and 11 & 0.4 \\
\hline 6 and 9 & 0.35 \\
\hline 7 and 8 & 0.88 \\
\hline $7^{1}$ and $8^{1}$ & 0.19 \\
\hline $7^{2}$ and $8^{2}$ & 0.02 \\
\hline 10 and 5 & 0.47 \\
\hline $10^{1}$ and $5^{1}$ & 0.06 \\
\hline $10^{2}$ and $5^{2}$ & 0.09 \\
\hline $10^{3}$ and $5^{3}$ & 0.14 \\
\hline $10^{4}$ and $5^{4}$ & 0 \\
\hline $10^{5}$ and $5^{5}$ & 0 \\
\hline $10^{6}$ and $5^{6}$ & 0.04 \\
\hline 15 and 20 & 0.19 \\
\hline $15^{1}$ and $20^{1}$ & 0 \\
\hline $15^{2}$ and $20^{2}$ & 0.3 \\
\hline $15^{3}$ and $20^{3}$ & 0 \\
\hline $15^{4}$ and $20^{4}$ & 0 \\
\hline $15^{5}$ and $20^{5}$ & 0 \\
\hline $15^{6}$ and $20^{6}$ & 1.3 \\
\hline 16 and 19 & 0.52 \\
\hline 17 and 18 & 0.2 \\
\hline $17^{1}$ and $18^{1}$ & 0.12 \\
\hline $17^{2}$ and $18^{2}$ & 0.18 \\
\hline 21 and 23 & 1.67 \\
\hline
\end{tabular}




\section{SUPPORTING INFORIMATION}

Table S2. Comparison of ${ }^{13} \mathrm{C}$ and ${ }^{15} \mathrm{~N}$ atom resonances a) $\alpha_{4}-\mathbf{P} \cdot \mathbf{B S A}{ }^{[20]} \alpha_{4}-\mathbf{P} \cdot \mathbf{1 0 C S A}(\mathbf{S}), \alpha_{4}-\mathbf{P} \cdot \mathbf{1 0 C S A}(\mathbf{S R})$ and, b) $\alpha \beta \alpha \beta-\mathbf{P} \cdot \mathbf{B S A}$, ${ }^{[20]} \alpha \beta \alpha \beta-\mathbf{P} \cdot 10 \mathrm{CSA}(\mathbf{S}), \alpha \beta \alpha \beta-$ P.10CSA(SR) complexes. The signal positions were determined using various NMR techniques $\left(\mathrm{CD}_{3} \mathrm{CN}\right)$ recorded with 20 eq. of analyte.

a)

\begin{tabular}{|c|c|c|c|}
\hline Pos. & BSA & $\begin{array}{l}\text { CSA-SR } \\
\delta C \text { or } \delta N\end{array}$ & CSA-S \\
\hline 1 & 143.2 & 143.9 & 141.9 \\
\hline 2 & 142.2 & 145.0 & 143.7 \\
\hline $2^{1}$ & 18.2 & 19.2 & 19.2 \\
\hline $2^{2}$ & 16.2 & 16.1 & 16.2 \\
\hline 3 & 142.2 & 145.0 & 143.7 \\
\hline $3^{1}$ & 18.2 & 19.2 & 19.2 \\
\hline $3^{2}$ & 16.2 & 16.1 & 16.1 \\
\hline 4 & 143.2 & 143.9 & 141.8 \\
\hline 5 & 114.1 & 113.8 & 113.8 \\
\hline $5^{1}$ & 131.7 & 131.6 & 131.6 \\
\hline $5^{2}$ & 133.0 & 133.5 & 133.4 \\
\hline $5^{3}$ & 126.2 & 126.3 & 126.2 \\
\hline $5^{4}$ & 134.3 & 134.1 & 134.0 \\
\hline $5^{5}$ & 130.7 & 130.5 & 130.5 \\
\hline $5^{6}$ & 140.6 & 141.0 & 140.9 \\
\hline 6 & 144.9 & 143.8 & 144.9 \\
\hline 7 & 144.8 & 141.9 & 143.9 \\
\hline $7^{1}$ & 20.4 & 20.4 & 19.2 \\
\hline $7^{2}$ & 15.0 & 15.7 & 15.7 \\
\hline 8 & 144.8 & 141.9 & 143.9 \\
\hline $8^{1}$ & 20.4 & 20.4 & 19.2 \\
\hline $8^{2}$ & 15.0 & 15.7 & 15.6 \\
\hline 9 & 144.9 & 143.8 & 145.2 \\
\hline 10 & 114.1 & 113.8 & 113.8 \\
\hline $10^{1}$ & 131.7 & 131.6 & 131.7 \\
\hline $10^{2}$ & 133.0 & 133.5 & 133.5 \\
\hline $10^{3}$ & 126.2 & 126.3 & 126.3 \\
\hline $10^{4}$ & 134.3 & 134.1 & 134.1 \\
\hline $10^{5}$ & 130.7 & 130.5 & 130.5 \\
\hline $10^{6}$ & 140.6 & 141.0 & 141.1 \\
\hline 11 & 143.2 & 143.9 & 141.9 \\
\hline 12 & 142.2 & 145.0 & 143.7 \\
\hline
\end{tabular}

$$
a_{4}-\mathrm{P}
$$

Pos.

\section{BSA}

BSA CSA-SR CSA-S CSA-SR
$\overline{O C}$ or $\bar{\delta} \mathrm{N}$ $\begin{array}{lll}18.2 & 19.2 \quad 19.2\end{array}$ $\begin{array}{lll}16.2 & 16.1 & 16.2\end{array}$ $\begin{array}{lll}142.2 & 145.0 & 143.7\end{array}$ $\begin{array}{lll}18.2 & 19.2 & 19.2\end{array}$ $\begin{array}{lll}16.2 & 16.1 & 16.1\end{array}$ $\begin{array}{lll}143.2 & 143.9 & 141.8\end{array}$ $\begin{array}{lll}114.1 & 113.8 & 113.8\end{array}$ $131.7 \quad 131.6 \quad 131.6$ $\begin{array}{lll}133.0 & 133.5 & 133.4\end{array}$ $\begin{array}{lll}126.2 & 126.3 & 126.2\end{array}$ $\begin{array}{lll}134.3 & 134.1 & 134.0\end{array}$ $\begin{array}{lll}130.7 & 130.5 & 130.5\end{array}$ $\begin{array}{lll}140.6 & 141.0 & 140.9\end{array}$ $\begin{array}{lll}144.9 & 143.8 & 144.9\end{array}$ $\begin{array}{lll}144.8 & 141.9 & 143.9\end{array}$ $\begin{array}{lll}20.4 & 20.4 & 19.2\end{array}$ $\begin{array}{lll}15.0 & 15.7 & 15.7\end{array}$ $\begin{array}{lll}144.8 & 141.9 & 143.9\end{array}$ $20.4 \quad 20.4 \quad 19.2$ $\begin{array}{lll}15.0 & 15.7 & 15.6\end{array}$ $\begin{array}{lll}144.9 & 143.8 & 145.2\end{array}$ $\begin{array}{lll}114.1 & 113.8 & 113.8\end{array}$ $\begin{array}{lll}131.7 & 131.6 & 131.7\end{array}$ $\begin{array}{lll}133.0 & 133.5 & 133.5\end{array}$ $\begin{array}{lll}126.2 & 126.3 & 126.3\end{array}$ $\begin{array}{lll}134.3 & 134.1 & 134.1\end{array}$ $\begin{array}{lll}130.7 & 130.5 & 130.5\end{array}$ $\begin{array}{lll}140.6 & 141.0 & 141.1\end{array}$ $\begin{array}{lll}125.8 & 127.9 & 127.7\end{array}$ $\begin{array}{lll}125.1 & 125.8 & 126.1\end{array}$ $\begin{array}{lll}125.8 & 127.9 & 127.7\end{array}$ $125.1 \quad 125.8 \quad 126.1$ b)

\begin{tabular}{|c|c|c|c|c|c|c|c|}
\hline Pos. & BSA & $\begin{array}{l}\text { CSA-SR } \\
\delta C \text { or } \delta N\end{array}$ & CSA-S & Pos. & BSA & $\begin{array}{l}\text { CSA-SR } \\
\delta C \text { or } \delta N\end{array}$ & CSA-S \\
\hline 1 & 143.6 & 145.0 & 145.3 & $12^{1}$ & 19.8 & 20.5 & 20.2 \\
\hline 2 & 143.8 & 142.7 & 142.7 & $12^{2}$ & 15.3 & 15.8 & 15.8 \\
\hline $2^{1}$ & 19.8 & 20.5 & 20.2 & 13 & 144.0 & 142.5 & 142.2 \\
\hline $2^{2}$ & 15.3 & 15.8 & 15.8 & $13^{1}$ & 20.6 & 19.7 & 19.7 \\
\hline 3 & 144.0 & 142.5 & 143.0 & $13^{2}$ & 15.6 & 16.1 & 16.1 \\
\hline $3^{1}$ & 20.6 & 19.7 & 19.7 & 14 & 145.0 & 143.9 & 143.9 \\
\hline $3^{2}$ & 15.6 & 16.1 & 16.1 & 15 & 113.1 & 113.2 & 113.2 \\
\hline 4 & 145.0 & 143.9 & 143.9 & $15^{1}$ & 132.1 & 132.2 & 132.5 \\
\hline 5 & 113.1 & 113.2 & 113.1 & $15^{2}$ & 132.9 & 134.6 & 133.9 \\
\hline $5^{1}$ & 132.1 & 132.2 & 132.2 & $15^{3}$ & 126.0 & 125.8 & 126.1 \\
\hline $5^{2}$ & 132.9 & 134.6 & 133.9 & $15^{4}$ & 134.4 & 133.8 & 133.9 \\
\hline $5^{3}$ & 126.0 & 125.8 & 125.9 & $15^{5}$ & 130.9 & 129.9 & 130.3 \\
\hline $5^{4}$ & 134.4 & 133.8 & 133.9 & $15^{6}$ & 140.1 & 140.3 & 133.9 \\
\hline $5^{5}$ & 130.9 & 129.9 & 130.1 & 16 & 143.6 & 145.0 & 145.3 \\
\hline $5^{6}$ & 140.1 & 140.3 & 133.9 & 17 & 143.8 & 142.7 & 142.7 \\
\hline 6 & 143.6 & 145.0 & 144.8 & $17^{1}$ & 19.8 & 20.5 & 20.2 \\
\hline 7 & 143.8 & 142.7 & 142.8 & $17^{2}$ & 15.3 & 15.8 & 15.8 \\
\hline $7^{1}$ & 19.8 & 20.5 & 20.2 & 18 & 144.0 & 142.5 & 143.0 \\
\hline $7^{2}$ & 15.3 & 15.8 & 15.8 & $18^{1}$ & 20.6 & 19.7 & 19.7 \\
\hline 8 & 144.0 & 142.5 & 142.2 & $18^{2}$ & 15.6 & 16.1 & 16.1 \\
\hline $8^{1}$ & 20.6 & 19.7 & 19.7 & 19 & 145.0 & 143.9 & 143.9 \\
\hline $8^{2}$ & 15.6 & 16.1 & 16.1 & 20 & 113.1 & 113.2 & 113.1 \\
\hline 9 & 145.0 & 143.9 & 143.9 & $20^{1}$ & 132.1 & 132.2 & 132.2 \\
\hline 10 & 113.1 & 113.2 & 113.2 & $20^{2}$ & 132.9 & 134.6 & 133.9 \\
\hline $10^{1}$ & 132.1 & 132.2 & 132.5 & $20^{3}$ & 126.0 & 125.8 & 125.9 \\
\hline $10^{2}$ & 132.9 & 134.6 & 133.9 & $20^{4}$ & 134.4 & 133.8 & 133.9 \\
\hline $10^{3}$ & 126.0 & 125.8 & 126.1 & $20^{5}$ & 130.9 & 129.9 & 130.1 \\
\hline $10^{4}$ & 134.4 & 133.8 & 133.9 & $20^{6}$ & 140.1 & 140.3 & 133.9 \\
\hline $10^{5}$ & 130.9 & 129.9 & 130.3 & 21 & 126.5 & 129.0 & 129.2 \\
\hline $10^{6}$ & 140.1 & 140.3 & 133.9 & 22 & 126.5 & 129.0 & 128.2 \\
\hline 11 & 143.6 & 145.0 & 144.8 & 23 & 126.5 & 129.0 & 128.2 \\
\hline 12 & 143.8 & 142.7 & 142.8 & 24 & 126.5 & 129.0 & 129.2 \\
\hline
\end{tabular}


Table S3. Comparison of the $\Delta \sigma_{\max }(\mathrm{ppm})$ in ${ }^{13} \mathrm{C},{ }^{15} \mathrm{~N}$ and ${ }^{1} \mathrm{H}$ atoms of $\alpha_{2} \beta_{2}-\mathbf{P} \cdot 10 \mathrm{CSA}(\mathbf{S}), \alpha_{4}-\mathbf{P} \cdot 10 \mathrm{CSA}(\mathbf{S})$, and $\alpha \beta \alpha \beta$-P.10CSA(S) recorded with 20 eq. of analyte. The signal positions were determined using 2D NMR techniques $\left(\mathrm{CD}_{3} \mathrm{CN}\right)$. On the right side, illustration of the $\alpha_{2} \beta_{2}-\mathbf{P}, \alpha_{4}-\mathbf{P}$, and $\alpha \beta \alpha \beta-\mathbf{P}$ with highlighted positions (blue and red represents the splitting signals between them), dashed green line - mirror planes, yellow dotted lines inversion points.

\begin{tabular}{|c|c|c|c|c|c|c|c|c|}
\hline \multicolumn{3}{|c|}{$\alpha_{2} \beta_{2}-\mathrm{P}-10 \mathrm{CSA}(\mathrm{S})$} & \multicolumn{3}{|c|}{$\alpha_{4}-\mathrm{P}-10 \operatorname{CSA}(\mathrm{S})$} & \multicolumn{3}{|c|}{$\alpha \beta \alpha \beta-\mathbf{P}-10 \mathrm{CSA}(\mathrm{S})$} \\
\hline Pos. & $\begin{array}{c}\Delta \delta C \text { or } \\
\Delta \delta \mathrm{N}\end{array}$ & $\Delta \delta \mathrm{H}$ & Pos. & \begin{tabular}{|l}
$\Delta \delta \mathrm{C}$ or \\
$\Delta \delta \mathrm{N}$
\end{tabular} & $\Delta \delta \mathrm{H}$ & Pos. & $\begin{array}{c}\Delta \delta C \text { or } \\
\Delta \delta N\end{array}$ & $\Delta \delta \mathrm{H}$ \\
\hline 1 and 14 & 0.98 & & 1 and 4 & 0.23 & & 1 and 11 & 0.47 & \\
\hline 2 and 13 & 0.59 & & 2 and 3 & 0.01 & & 2 and 12 & 0.09 & \\
\hline $2^{1}$ and $13^{1}$ & 0.26 & 0.03 & $2^{1}$ and $3^{1}$ & 0 & 0 & $2^{1}$ and $12^{1}$ & 0 & 0.02 \\
\hline $2^{2}$ and $13^{2}$ & 0.22 & 0.16 & $2^{2}$ and $3^{2}$ & 0.11 & 0.08 & $2^{2}$ and $12^{2}$ & 0 & 0.22 \\
\hline 3 and 12 & 0.22 & & 6 and 9 & 0.1 & & 4 and 14 & 0 & \\
\hline $3^{1}$ and $12^{1}$ & 0.12 & 0.09 & 7 and 8 & 0.08 & & 3 and 13 & 0.8 & \\
\hline $3^{2}$ and $12^{2}$ & 0.12 & 0.03 & $7^{1}$ and $8^{1}$ & 0 & 0 & $3^{1}$ and $13^{1}$ & 0 & 0.1 \\
\hline 4 and 11 & 0.4 & & $7^{2}$ and $8^{2}$ & 0.03 & 0 & $3^{2}$ and $13^{2}$ & 0.03 & 0.03 \\
\hline 6 and 9 & 0.35 & & 10 and 5 & 0.06 & & 6 and 16 & 0.47 & \\
\hline 7 and 8 & 0.88 & & $10^{1}$ and $5^{1}$ & 0.05 & & 7 and 17 & 0.09 & \\
\hline $7^{1}$ and $8^{1}$ & 0.19 & 0.03 & $10^{2}$ and $5^{2}$ & 0.18 & & $7^{1}$ and $17^{1}$ & 0 & 0.02 \\
\hline $7^{2}$ and $8^{2}$ & 0.02 & 0.07 & $10^{3}$ and $5^{3}$ & 0.07 & 0.07 & $7^{2}$ and $17^{2}$ & 0 & 0.22 \\
\hline 10 and 5 & 0.47 & & $10^{4}$ and $5^{4}$ & 0.06 & 0.01 & 9 and 19 & 0 & \\
\hline $10^{1}$ and $5^{1}$ & 0.06 & & $10^{5}$ and $5^{5}$ & 0 & 0 & 8 and 18 & 0.8 & \\
\hline $10^{2}$ and $5^{2}$ & 0.09 & & $10^{6}$ and $5^{6}$ & 0.19 & 0.01 & $8^{1}$ and $18^{1}$ & 0 & 0.1 \\
\hline $10^{3}$ and $5^{3}$ & 0.14 & & 13 and 12 & 0.23 & & $8^{2}$ and $18^{2}$ & 0.03 & 0.03 \\
\hline $10^{4}$ and $5^{4}$ & 0 & & $13^{1}$ and $12^{1}$ & 0.01 & & 15 and 5 & 0.1 & \\
\hline $10^{5}$ and $5^{5}$ & 0 & & $13^{2}$ and $12^{2}$ & 0 & 0 & $15^{1}$ and $5^{1}$ & 0.31 & \\
\hline $10^{6}$ and $5^{6}$ & 0.04 & 0.2 & 11 and 14 & 0.11 & 0.08 & $15^{2}$ and $5^{2}$ & 0 & \\
\hline 15 and 20 & 0.19 & & 16 and 19 & 0.1 & & $15^{3}$ and $5^{3}$ & 0.16 & 0.05 \\
\hline $15^{1}$ and $20^{1}$ & 0 & & 17 and 18 & 0.08 & & $15^{4}$ and $5^{4}$ & 0 & 0 \\
\hline $15^{2}$ and $20^{2}$ & 0.3 & & $17^{1}$ and $18^{1}$ & 0 & 0 & $15^{5}$ and $5^{5}$ & 0.22 & 0 \\
\hline $15^{3}$ and $20^{3}$ & 0 & & $17^{2}$ and $18^{2}$ & 0.03 & 0 & $15^{6}$ and $5^{6}$ & 0 & 0.16 \\
\hline $15^{4}$ and $20^{4}$ & 0 & & 15 and 20 & 0.06 & & 10 and 20 & 0.1 & \\
\hline $15^{5}$ and $20^{5}$ & 0 & & $15^{1}$ and $20^{1}$ & 0.05 & & $10^{1}$ and $20^{1}$ & 0.31 & \\
\hline $15^{6}$ and $20^{6}$ & 1.3 & 0.17 & $15^{2}$ and $20^{2}$ & 0.18 & & $10^{2}$ and $20^{2}$ & 0 & \\
\hline 16 and 19 & 0.52 & & $15^{3}$ and $20^{3}$ & 0.07 & 0.07 & $10^{3}$ and $20^{3}$ & 0.16 & 0.05 \\
\hline 17 and 18 & 0.2 & & $15^{4}$ and $20^{4}$ & 0.06 & 0.01 & $10^{4}$ and $20^{4}$ & 0 & 0 \\
\hline $17^{1}$ and $18^{1}$ & 0.12 & 0.09 & $15^{5}$ and $20^{5}$ & 0 & 0 & $10^{5}$ and $20^{5}$ & 0.22 & 0 \\
\hline $17^{2}$ and $18^{2}$ & 0.18 & 0.03 & $15^{6}$ and $20^{6}$ & 0.19 & 0.01 & $10^{6}$ and $20^{6}$ & 0 & 0.16 \\
\hline 21 and 23 & 1.67 & 0.66 & 21 and 23 & 0 & 0 & 21 and 23 & 1.03 & 0.52 \\
\hline 22 and 24 & 0 & 0 & 22 and 24 & 0 & 0 & 22 and 24 & 1.03 & 0.52 \\
\hline
\end{tabular}

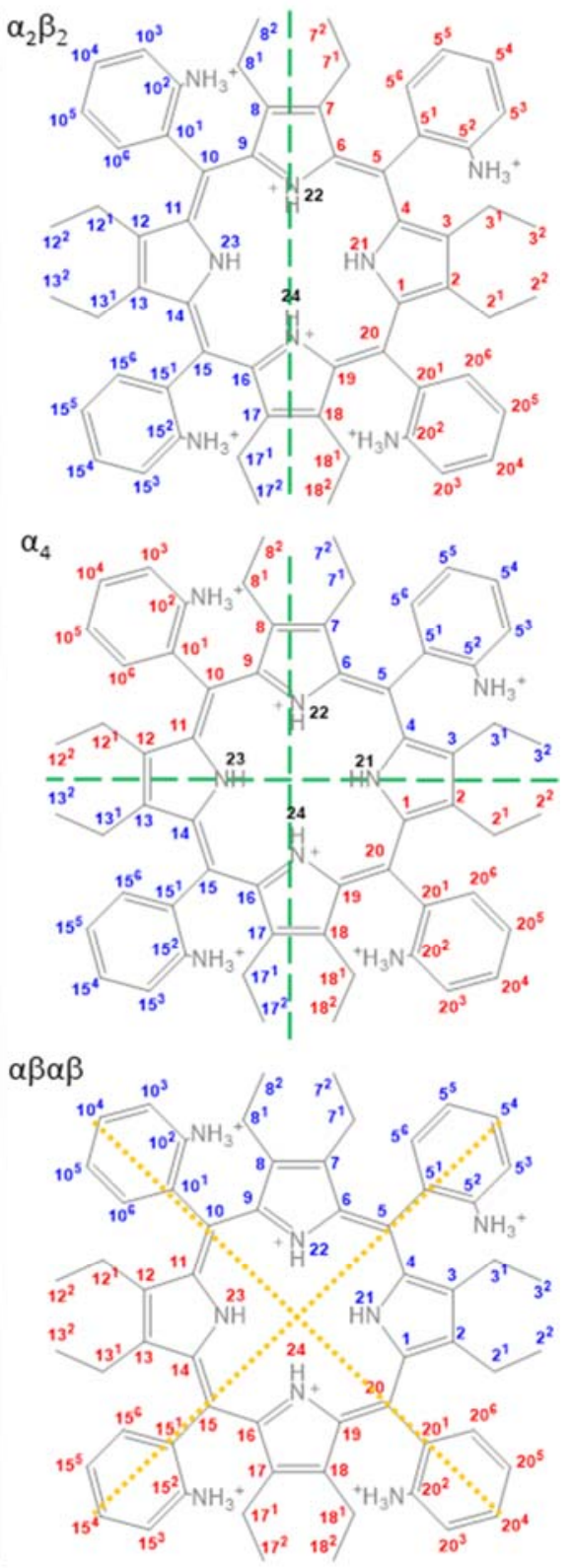




\section{SUPPORTING INFORVATION}

Structural Determination of $\alpha_{2} \beta_{2}-\mathrm{P}\left[\mathrm{SO}_{4}{ }^{2-}\right]\left[\mathrm{HSO}_{4}{ }^{-}\right]_{4}$

Table S4: Details of XRD data refinement of $\alpha_{2} \beta_{2}-\mathrm{P}\left[\mathrm{SO}_{4}{ }^{2-}\right]\left[\mathrm{HSO}_{4}{ }^{-}\right]_{4}$

\begin{tabular}{|c|c|}
\hline Compound & $\alpha_{2} \beta_{2}-\mathrm{P}\left[\mathrm{SO}_{4}^{2-}\right]\left[\mathrm{HSO}_{4}^{-}\right]_{4}$ \\
\hline Internal code & KN007 \\
\hline$C C D C \#$ & 2143572 \\
\hline Empirical formula & $\mathrm{C}_{60} \mathrm{H}_{84.38} \mathrm{~N}_{8} \mathrm{O}_{23.95} \mathrm{~S}_{5}$ \\
\hline Formula weight & 1461.23 \\
\hline Temperature/K & $100(2)$ \\
\hline Crystal system & Monoclinic \\
\hline Space group & $\mathrm{P} 2{ }_{1} / \mathrm{n}$ \\
\hline$a / \AA$ & $18.7594(9)$ \\
\hline$b / \AA$ & $16.0888(8)$ \\
\hline$c / \AA$ & 24.9988(12) \\
\hline$\alpha /^{\circ}$ & 90 \\
\hline$\beta /^{\circ}$ & $90.962(2)$ \\
\hline$y /{ }^{\circ}$ & 90 \\
\hline Volume $/ \AA^{3}$ & $7544.0(6)$ \\
\hline$Z$ & 4 \\
\hline$D_{\text {calc }} \mathrm{g} / \mathrm{cm}^{3}$ & 1.287 \\
\hline$\mu / m m^{-1}$ & 2.066 \\
\hline$F(000)$ & 3088.0 \\
\hline Crystal size $/ \mathrm{mm}^{3}$ & $0.15 \times 0.1 \times 0.07$ \\
\hline Radiation & CuKa \\
\hline Wavelength/Å & 1.54178 \\
\hline $2 \theta /^{\circ}$ & 5.844 to 140.326 \\
\hline Reflections collected & 94031 \\
\hline Independent reflections & 14243 \\
\hline$R_{\text {int }}$ & 0.0578 \\
\hline$R_{\text {sigma }}$ & 0.0345 \\
\hline Restraints & 3307 \\
\hline Parameters & 1354 \\
\hline GooF & 1.104 \\
\hline$R_{1}[I>2 \sigma(I)]$ & 0.1354 \\
\hline$w R_{2}[I>2 \sigma(I)]$ & 0.3912 \\
\hline$R_{1}$ [all data] & 0.1537 \\
\hline$w R_{2}$ [all data] & 0.4253 \\
\hline Largest peak/e $\AA^{-3}$ & 1.73 \\
\hline Deepest hole/e $\AA^{-3}$ & -0.99 \\
\hline
\end{tabular}




\section{SUPPORTING INFORIMATION}

\begin{tabular}{|c|c|c|c|c|c|c|c|c|}
\hline basis & $\Delta_{\text {ip }}$ & $\delta_{\text {ip }}$ & $\mathbf{B}_{2 \mathrm{~g}}$ & $\mathbf{B}_{1 \mathrm{~g}}$ & $E_{u}(x)$ & $E_{u}(y)$ & $\mathbf{A}_{1 \mathrm{~g}}$ & $\mathbf{A}_{2 \mathrm{~g}}$ \\
\hline $\min$. & 0.75 & 0.18 & -0.02 & 0.11 & -0.02 & 0.00 & -0.74 & 0.01 \\
\hline \multirow[t]{2}{*}{ ext. } & 0.88 & 0.13 & -0.02 & 0.11 & -0.02 & 0.00 & -0.74 & 0.01 \\
\hline & & & 0.01 & 0.01 & -0.03 & 0.01 & -0.46 & 0.01 \\
\hline \multirow[t]{11}{*}{ total } & 1.11 & 0.00 & -0.02 & 0.11 & -0.02 & 0.00 & -0.71 & 0.01 \\
\hline & & & 0.01 & 0.01 & -0.03 & 0.01 & -0.45 & 0.01 \\
\hline & & & 0.01 & -0.01 & -0.01 & -0.01 & 0.71 & 0.00 \\
\hline & & & 0.00 & 0.00 & -0.02 & 0.02 & 0.00 & 0.00 \\
\hline & & & 0.01 & 0.01 & 0.01 & -0.01 & -0.07 & 0.00 \\
\hline & & & 0.00 & 0.00 & 0.01 & -0.01 & -0.07 & \\
\hline & & & & & -0.01 & 0.02 & & \\
\hline & & & & & 0.00 & 0.00 & & \\
\hline & & & & & 0.00 & -0.02 & & \\
\hline & & & & & 0.00 & 0.00 & & \\
\hline & & & & & 0.00 & 0.01 & & \\
\hline comp. & 1.11 & 0.00 & 0.02 & 0.11 & 0.04 & 0.04 & 1.10 & 0.02 \\
\hline basis & $\Delta_{\text {oop }}$ & $\delta_{\text {oop }}$ & $\mathrm{B}_{2 \mathrm{u}}$ & $\mathbf{B}_{1 \mathrm{u}}$ & $A_{2 u}$ & $E_{g}(x)$ & $E_{g}(y)$ & $\mathbf{A}_{1 \mathrm{u}}$ \\
\hline $\min$. & 3.88 & 0.11 & -3.88 & -0.02 & 0.02 & 0.02 & -0.01 & 0.02 \\
\hline \multirow[t]{2}{*}{ ext. } & 3.92 & 0.00 & -3.87 & -0.02 & 0.02 & 0.02 & -0.01 & 0.02 \\
\hline & & & 0.65 & -0.01 & -0.02 & -0.04 & 0.00 & 0.00 \\
\hline \multirow[t]{5}{*}{ total } & 3.92 & 0.00 & -3.87 & -0.02 & 0.02 & 0.02 & -0.01 & 0.02 \\
\hline & & & 0.65 & -0.01 & -0.02 & -0.04 & 0.00 & 0.00 \\
\hline & & & 0.07 & -0.01 & 0.00 & 0.01 & -0.03 & \\
\hline & & & & & & -0.01 & 0.00 & \\
\hline & & & & & & -0.01 & 0.00 & \\
\hline & & & 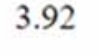 & - & 3 & 0.04 & 0.03 & 0.02 \\
\hline
\end{tabular}
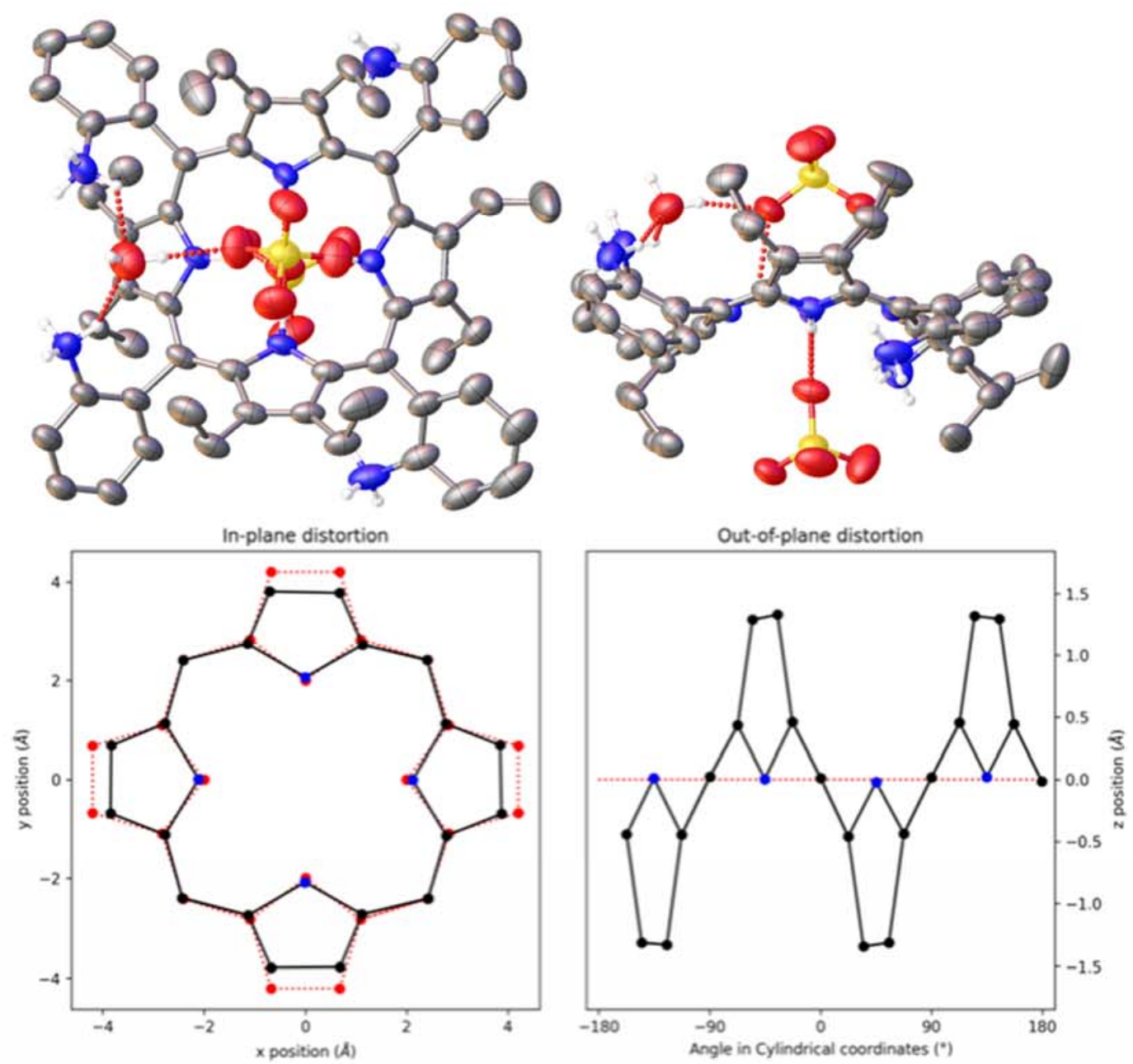

Figure S13. Left: in-plane (ip) and out-of-plane (oop) NSD results of $\left.\alpha_{2} \beta_{2}-\mathrm{P}_{\left.\mathrm{SO}_{4}{ }^{2}\right]}\right]\left[\mathrm{HSO}_{4}{ }^{-}\right] 4$; Right bottom: out-of-plane and in-plane skeletal plots of the porphyrin $\alpha_{2} \beta_{2}{ }^{-}$ $\mathrm{P}\left[\mathrm{SO}_{4}{ }^{2}\right]\left[\mathrm{HSO}_{4}{ }^{-}\right]_{4}$ core. Porphyrin $\alpha_{2} \beta_{2}-\mathrm{P}$ is represented in black $(\mathrm{C})$ and blue $(\mathrm{N})$, with the reference structure (CuTPP) in red dotted lines. ${ }^{[2]}$ Right top: top view and sideview of $\alpha_{2} \beta_{2}-\mathrm{P}-\mathrm{SO}_{4}$ structure. Non-essential hydrogens, majority of counter anions and solvent molecules omitted for clarity, thermal ellipsoids shown at $50 \%$ probability. 


\section{SUPPORTING INFORMATION}

\section{Computational Analysis}

\section{Geometry analysis}

Based on the crystallographic data we concluded that one porphyrin cation binds two camphorsulfonic acid molecules. Using this ratio and the $\alpha_{2} \beta_{2}-\mathrm{P}\left[\mathrm{SO}_{4}{ }^{2}\right]\left[\mathrm{HSO}_{4}{ }^{-}\right]_{4}$ crystal data the corresponding host-guest complexes were built with subsequent conformation search. According to the Boltzmann distribution, in acetonitrile one major conformer A $(90 \%)$ and one minor conformer $\mathbf{B}$ (up to $10 \%$ ) being higher in energy by $1.30 \mathrm{kcal} / \mathrm{mol}$ are presented (Table S5).

In all host-guest complexes, the porphyrin plane is significantly distorted (the $\mathrm{C}_{\beta}-\mathrm{C}_{\beta}-\mathrm{C}_{\beta o p p}-\mathrm{C}_{\beta \text { opp }}$ angles varying in the range of $0^{\circ}-25^{\circ}$ and $\mathrm{N}-\mathrm{C}_{\alpha}-\mathrm{C}_{\alpha}-\mathrm{N}$ angles varying in the range of $37^{\circ}-48^{\circ}$ ) because of a steric hindrance between the peripheral substituents (for NSD profile check Figure S13). This deformation results in appearance of two cavities on both sides of the porphyrin macrocycle, which differ by the position of $\mathrm{NH}_{3}{ }^{+}$groups. In one cavity the ammonia groups are placed on the same side of the cavity and in another - on the opposite sides (Figure S14a), and the distance between two nitrogen atoms increases from $4.899 \AA$ to $8.7174 \AA$, respectively. The difference in the position of $\mathrm{NH}_{3}{ }^{+}$groups results in a non-identical mode of the binding of two camphorsulfonic acids ("standing" and "lying"). Both the NBO and AIMALL analysis showed that the guest molecules interact with the porphyrin cation through the formation of four $\mathrm{H}$-bonds, which cause significant elongation of the $\mathrm{N}-\mathrm{H}$ bonds by $0.08-0.03$ $\AA$ in the case of $\mathrm{NH}_{3}{ }^{+}$groups and by $0.03 \AA$ in the case of inner core protons for the major conformer $\mathbf{A}$ (Figure S14b, Tables S6 and S7).
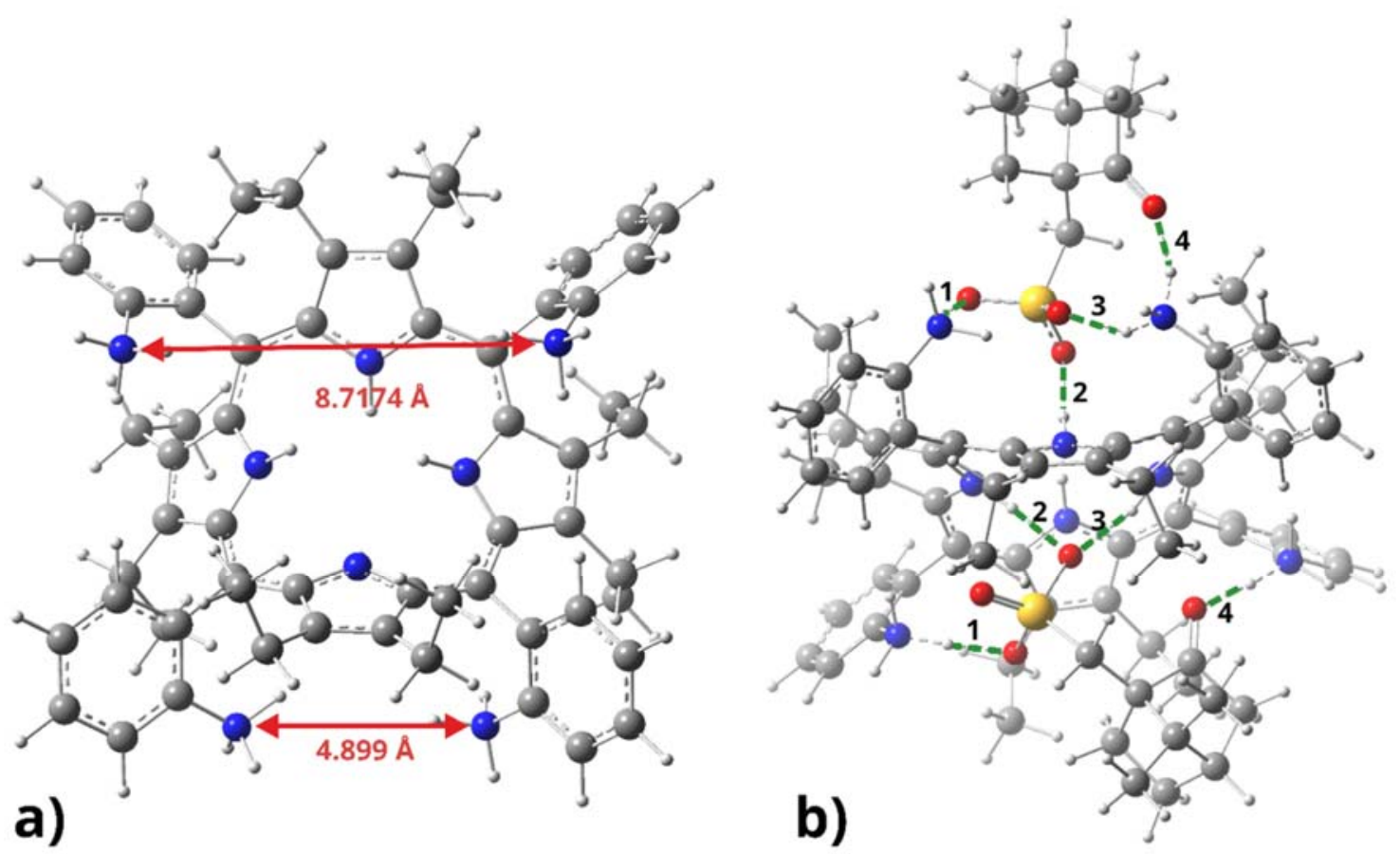

Figure S14. a) Position of $\mathrm{NH}_{3}{ }^{+}$groups in porphyrin cation; b) major conformer $\mathbf{A}$ of the $\alpha_{2} \beta_{2}-\mathbf{P} \cdot 10 \mathrm{CSA}(\mathbf{R})$ complex, where hydrogen bonds formed between porphyrin and two guest molecules are shown by the green dashed lines and numbered. 


\section{SUPPORTING INFORVATION}

Table S5. Relative energies and Boltzmann distribution of $\alpha_{2} \beta_{2}-\mathbf{P} \cdot 10 \mathrm{CSA}(\mathbf{R})$ conformers.

\begin{tabular}{|c|c|c|c|c|c|}
\hline Conf. & $\begin{array}{c}\text { El. energy, Hartree } \\
\text { (def2-SVP) }\end{array}$ & $\begin{array}{c}\text { El. energy, Hartree } \\
\text { (def2-SVP) }\end{array}$ & $\begin{array}{c}\text { Gibbs Free } \\
\text { energy } \\
\text { correction, } \\
\text { Hartree }\end{array}$ & $\begin{array}{c}\text { Relative Gibbs } \\
\text { Free energy, } \\
\text { kcal/mol }\end{array}$ & $\begin{array}{c}\text { Boltzmann } \\
\text { distribution, \% }\end{array}$ \\
\hline A & $-4942,80946$ & $-4947,6688$ & 1,534213 & 0 & 90 \\
\hline B & $-4942,80783$ & $-4947,66664$ & 1,534117 & 1,3 & 10 \\
\hline C & $-4942,79772$ & $-4947,65773$ & 1,533141 & 6,28 & 0 \\
\hline D & $-4942,79733$ & $-4947,6544$ & 1,534908 & 9,48 & 0 \\
\hline E & $-4942,79347$ & $-4947,65221$ & 1,532854 & 9,56 & 0 \\
\hline F & $-4942,79077$ & $-4947,64779$ & 1,536709 & 14,75 & 0 \\
\hline G & $-4942,77487$ & $-4947,63394$ & 1,532741 & 20,96 & 0 \\
\hline H & $-4942,7644$ & $-4947,6243$ & 1,532105 & 26,6 & 0 \\
\hline
\end{tabular}

Table S6. Perturbation theory energy analysis of A conformer of $\alpha_{2} \beta_{2}-\mathbf{P} \cdot \mathbf{1 0 C S A}(\mathbf{R})$ complex.

\begin{tabular}{|c|c|c|c|c|c|c|}
\hline \multirow{2}{*}{\begin{tabular}{|c} 
Num. \\
of \\
$\mathrm{H}-$ \\
bond
\end{tabular}} & \multicolumn{3}{|c|}{ "standing" guest } & \multicolumn{3}{|c|}{ "lying" guest } \\
\hline & Donor NBO & Acceptor NBO & $\begin{array}{l}\mathrm{E}(2), \\
\text { kcal/ } \\
\text { mol }\end{array}$ & Donor NBO (I) & Acceptor NBO (j) & $\begin{array}{l}\mathrm{E}(2), \\
\text { kcal/ } \\
\text { mol }\end{array}$ \\
\hline 1 & BD (1) O 138 - S 165 & $R Y^{*}(2) \mathrm{H} 103$ & 0.12 & $\mathrm{BD}(1) \mathrm{N} 127-\mathrm{H} 130$ & $R Y^{*}(3) 081$ & 0.06 \\
\hline & BD (1) O 138 - S 165 & $R Y^{*}(3) \mathrm{H} 103$ & 0.1 & $\mathrm{BD}(1) \mathrm{N} 127-\mathrm{H} 130$ & $R Y^{*}(9) 081$ & 0.06 \\
\hline & BD (1) O 138 - S 165 & $\mathrm{RY}^{*}(4) \mathrm{H} 103$ & 0.1 & $\mathrm{BD}(1) \mathrm{N} 127-\mathrm{H} 130$ & $\mathrm{BD}^{*}(1) \mathrm{O} 81-\mathrm{S} 169$ & 0.52 \\
\hline & $\mathrm{BD}(1) \mathrm{O} 138$ - S 165 & $R Y^{*}(5) \mathrm{H} 103$ & 0.07 & $\mathrm{BD}(1) \mathrm{N} 127-\mathrm{H} 130$ & $R Y^{*}(3) 081$ & 1.42 \\
\hline & BD (1) O 138 - S 165 & $\mathrm{BD}^{*}(1) \mathrm{N} 100-\mathrm{H} 103$ & 0.31 & BD (1) O81 - S 169 & $R Y^{*}(2) \mathrm{H} 130$ & 0.09 \\
\hline & CR (1) O 138 & $\mathrm{BD}^{*}(1) \mathrm{N} 100-\mathrm{H} 103$ & 0.84 & BD (1) 081 - S 169 & $\mathrm{RY}^{*}(3) \mathrm{H} 130$ & 0.09 \\
\hline & LP (1) O 138 & $R Y^{*}(1) H 103$ & 0.29 & BD (1) O81 - S 169 & $R Y^{*}(4) H 130$ & 0.18 \\
\hline & LP (1) O 138 & $\mathrm{RY}^{*}(3) \mathrm{H} 103$ & 0.14 & BD (1) 081 - S 169 & $\mathrm{BD}^{*}(1) \mathrm{N} 127-\mathrm{H} 130$ & 0.48 \\
\hline & LP (1) O 138 & $\mathrm{BD}^{*}(1) \mathrm{N} 100-\mathrm{H} 103$ & 10.53 & CR (1) 081 & $R Y^{*}(1) H 130$ & 0.06 \\
\hline & LP (2) O 138 & $\mathrm{RY}^{*}(6) \mathrm{H} 103$ & 0.15 & CR (1) 081 & $R Y^{*}(2) \mathrm{H} 130$ & 0.06 \\
\hline & LP (3) O 138 & $\mathrm{RY}^{*}(1) \mathrm{H} 103$ & 1.08 & CR (1) 081 & $\mathrm{BD}^{*}(1) \mathrm{N} 127-\mathrm{H} 130$ & 1.24 \\
\hline & LP (3) O 138 & $R Y^{*}(2) \mathrm{H} 103$ & 1.28 & CR (1) 081 & $R Y^{*}(1) H 130$ & 0.34 \\
\hline & LP (3) O 138 & $\mathrm{RY}^{*}(3) \mathrm{H} 103$ & 0.31 & CR (1) 081 & $\mathrm{RY}^{*}(2) \mathrm{H} 130$ & 0.06 \\
\hline & LP (3) O 138 & $R Y^{*}(4) \mathrm{H} 103$ & 0.25 & CR (1) 081 & $R Y^{*}(3) H 130$ & 0.06 \\
\hline & LP (3) O 138 & $\mathrm{RY}^{*}(5) \mathrm{H} 103$ & 0.08 & CR (1) 081 & $\mathrm{BD}^{*}(1) \mathrm{N} 127-\mathrm{H} 130$ & 11.71 \\
\hline & LP (3) O 138 & $\mathrm{RY}^{*}(8) \mathrm{H} 103$ & 0.07 & LP (2) O81 & $\mathrm{RY}^{*}(3) \mathrm{H} 130$ & 0.1 \\
\hline & LP (3) O 138 & $\mathrm{BD}^{*}(1) \mathrm{N} 100-\mathrm{H} 103$ & 33.97 & LP (2) O81 & $R Y^{*}(6) \mathrm{H} 130$ & 0.07 \\
\hline & $\mathrm{BD}^{*}(1) \mathrm{O} 138-\mathrm{S} 165$ & $\mathrm{BD}^{*}(1) \mathrm{N} 100-\mathrm{H} 103$ & 4.38 & LP (2) 081 & $\mathrm{RY}^{*}(7) \mathrm{H} 130$ & 0.05 \\
\hline & BD (1) N $100-\mathrm{H} 103$ & $\mathrm{RY}^{*}(2) \mathrm{O} 138$ & 0.07 & LP (2) O81 & $\mathrm{BD}^{*}(1) \mathrm{N} 127-\mathrm{H} 130$ & 0.32 \\
\hline & BD (1) N $100-\mathrm{H} 103$ & $\mathrm{BD}^{*}(1) \mathrm{O} 138-\mathrm{S} 165$ & 0.4 & LP (3) O81 & $R Y^{*}(1) \mathrm{H} 130$ & 0.65 \\
\hline & & & & LP (3) 081 & $\mathrm{RY}^{*}(2) \mathrm{H} 130$ & 2.32 \\
\hline & & & & LP (3) O81 & $R Y^{*}(3) \mathrm{H} 130$ & 0.13 \\
\hline & & & & LP (3) 081 & $\mathrm{RY}^{*}(4) \mathrm{H} 130$ & 0.23 \\
\hline & & & & LP (3) O81 & $R Y^{*}(5) \mathrm{H} 130$ & 0.16 \\
\hline & & & & LP (3) O81 & $\mathrm{RY}^{*}(8) \mathrm{H} 130$ & 0.06 \\
\hline & & & & LP (3) O81 & $\mathrm{BD}^{*}(1) \mathrm{N} 127-\mathrm{H} 130$ & 43.13 \\
\hline & & & & $\mathrm{BD}^{*}(1) \mathrm{O} 81$ - S 169 & $\begin{array}{l}\mathrm{BD}^{*}(1) \mathrm{N} 127-\mathrm{H} \\
130\end{array}$ & 5.51 \\
\hline & Total & & 54.54 & Total & & 69.16 \\
\hline & BD (1) S 165 - O 167 & $\mathrm{RY}^{*}(2) \mathrm{H} 53$ & 0.07 & BD (1) N50 - H51 & $R Y^{*}(2) 087$ & 0.11 \\
\hline
\end{tabular}




\begin{tabular}{|c|c|c|c|c|c|}
\hline BD (1) S 165 - 0167 & $\mathrm{RY}^{*}(4) \mathrm{H} 53$ & 0.05 & BD (1) N50 - H51 & $R Y^{*}(3) 087$ & 0.09 \\
\hline BD (1) S 165 - O 167 & $\mathrm{BD}^{*}(1) \mathrm{N} 52-\mathrm{H} 53$ & 0.35 & BD (1) N50 - H51 & $\mathrm{BD}^{*}(1) 087-\mathrm{S} 169$ & 0.18 \\
\hline CR (1) O 167 & $\mathrm{BD}^{*}(1) \mathrm{N} 52-\mathrm{H} 53$ & 0.56 & $\mathrm{BD}(1) \mathrm{O} 87$ - S 169 & $R Y^{*}(3) \mathrm{H} 51$ & 0.16 \\
\hline LP (1) O 167 & $R Y^{*}(1) \mathrm{H} 53$ & 0.12 & BD (1) 087 - S 169 & $R Y^{*}(6) \mathrm{H} 51$ & 0.11 \\
\hline LP (1) O 167 & $R Y^{\star}(2) H 53$ & 0.33 & BD (1) 087 - S 169 & $\mathrm{BD}^{\star}(1) \mathrm{N} 50-\mathrm{H} 51$ & 0.69 \\
\hline $\operatorname{LP}(1) O 167$ & $R Y^{\star}(3) \mathrm{H} 53$ & 0.09 & CR (1) 087 & $\mathrm{BD}^{*}(1) \mathrm{N} 50-\mathrm{H} 51$ & 0.71 \\
\hline $\operatorname{LP}(1) 0167$ & $\mathrm{BD}^{*}(1) \mathrm{N} 52-\mathrm{H} 53$ & 8.72 & LP (1) O87 & $R Y^{*}(1) H 51$ & 0.12 \\
\hline $\operatorname{LP}(2) \circ 167$ & $\mathrm{RY}^{*}(2) \mathrm{H} 53$ & 0.13 & LP (1) O87 & $R Y^{*}(2) H 51$ & 0.26 \\
\hline $\operatorname{LP}(2) \circ 167$ & $\mathrm{BD}^{*}(1) \mathrm{N} 52-\mathrm{H} 53$ & 0.88 & 3 LP (1) 087 & $\mathrm{BD}^{*}(1) \mathrm{N} 50-\mathrm{H} 51$ & 8.05 \\
\hline LP (3) O 167 & $R Y^{*}(1) H 53$ & 0.05 & LP (2) O87 & $R Y^{*}(7) \mathrm{H} 51$ & 0.07 \\
\hline $\operatorname{LP}(3) O 167$ & $R Y^{*}(2) \mathrm{H} 53$ & 2.3 & $3 \operatorname{LP}(3) 087$ & $R Y^{*}(1) H 51$ & 2.29 \\
\hline $\operatorname{LP}(3) O 167$ & $\mathrm{RY}^{*}(3) \mathrm{H} 53$ & 0.61 & LP (3) O87 & $R Y^{*}(2) H 51$ & 0.45 \\
\hline $\operatorname{LP}(3) O 167$ & $R Y^{*}(4) H 53$ & 0.09 & LP (3) O87 & $R Y^{*}(3) \mathrm{H} 51$ & 0.08 \\
\hline $\operatorname{LP}(3) \circ 167$ & $\mathrm{RY}^{*}(5) \mathrm{H} 53$ & 0.46 & LP (3) O87 & $R Y^{*}(4) H 51$ & 0.14 \\
\hline $\operatorname{LP}(3) \circ 167$ & $\mathrm{BD}^{*}(1) \mathrm{N} 52-\mathrm{H} 53$ & 16.92 & LP (3) 087 & $R Y^{*}(5) H 51$ & 0.59 \\
\hline$B D^{*}(1) S 165-0167$ & $\mathrm{BD}^{*}(1) \mathrm{N} 52-\mathrm{H} 53$ & 1.36 & LP (3) O87 & $\mathrm{BD}^{*}(1) \mathrm{N} 50-\mathrm{H} 51$ & 18.95 \\
\hline BD (1) N52 - H53 & $R Y^{*}(1) \bigcirc 167$ & 0.15 & $\mathrm{BD}^{*}(1) \mathrm{O} 87$ - S 169 & $\mathrm{BD}^{*}(1) \mathrm{N} 50-\mathrm{H} 51$ & 0.8 \\
\hline $\mathrm{BD}(1) \mathrm{N} 52-\mathrm{H} 53$ & $R Y^{\star}(2) \bigcirc 167$ & 0.06 & & & \\
\hline $\mathrm{BD}(1) \mathrm{N} 52-\mathrm{H} 53$ & $\mathrm{BD}^{*}(1) \mathrm{S} 165-\mathrm{O} 167$ & 0.18 & & & \\
\hline Total & & 33.48 & Total & & 33.85 \\
\hline 3 BD (1) S 165 - O 166 & $R Y^{*}(1) H 91$ & 0.36 & BD (1) N54 - H55 & $R Y^{*}(1) O 87$ & 0.16 \\
\hline $\mathrm{BD}(1) \mathrm{S} 165-\mathrm{O} 166$ & $\mathrm{RY}^{*}(3) \mathrm{H} 91$ & 0.24 & BD (1) N54 - H55 & $R Y^{*}(4) 087$ & 0.06 \\
\hline $\mathrm{BD}(1) \mathrm{S} 165-0166$ & $R Y^{*}(4) \mathrm{H} 91$ & 0.06 & BD (1) N54 - H55 & $\mathrm{BD}^{*}(1) \mathrm{O} 87$ - S 169 & 1.3 \\
\hline $\mathrm{BD}(1) \mathrm{S} 165-\mathrm{O} 166$ & $\mathrm{BD}^{*}(1) \mathrm{N} 90-\mathrm{H} 91$ & 0.08 & BD (1) O87 - S 169 & $R Y^{*}(1) H 55$ & 1.57 \\
\hline CR (1) O 166 & $\mathrm{BD}^{*}(1) \mathrm{N} 90-\mathrm{H} 91$ & 0.48 & BD (1) O87 - S 169 & $R Y^{\star}(2) \mathrm{H} 55$ & 0.22 \\
\hline LP (1) O 166 & $R Y^{*}(1) H 91$ & 0.52 & BD (1) 087 - S 169 & $R Y^{*}(3) \mathrm{H} 55$ & 0.63 \\
\hline LP (1) O 166 & $\mathrm{RY}^{*}(2) \mathrm{H} 91$ & 0.21 & BD (1) 087 - S 169 & $\mathrm{BD}^{*}(1) \mathrm{N} 54-\mathrm{H} 55$ & 0.06 \\
\hline LP (1) O 166 & $\mathrm{RY}^{*}(5) \mathrm{H} 91$ & 0.06 & CR (1) 087 & $\mathrm{BD}^{*}(1) \mathrm{N} 54-\mathrm{H} 55$ & 0.48 \\
\hline LP (1) O 166 & $\mathrm{BD}^{*}(1) \mathrm{N} 90-\mathrm{H} 91$ & 9.85 & LP (1) O87 & $R Y^{*}(1) \mathrm{H} 55$ & 1.93 \\
\hline $\operatorname{LP}(2) 0166$ & $\mathrm{BD}^{*}(1) \mathrm{N} 90-\mathrm{H} 91$ & 0.46 & LP (1) O87 & $R Y^{*}(2) H 55$ & 0.14 \\
\hline $\operatorname{LP}(3) \bigcirc 166$ & $R Y^{*}(1) H 91$ & 2.14 & LP (1) 087 & $R Y^{*}(3) \mathrm{H} 55$ & 0.06 \\
\hline LP (3) O 166 & $\mathrm{RY}^{*}(3) \mathrm{H} 91$ & 0.21 & LP (1) O87 & $B D^{\star}(1) N 54-H 55$ & 15.73 \\
\hline LP (3) O 166 & $R Y^{*}(5) \mathrm{H} 91$ & 0.12 & LP (2) O87 & $R Y^{*}(1) \mathrm{H} 55$ & 0.08 \\
\hline LP (3) O 166 & $\mathrm{BD}^{*}(1) \mathrm{N} 90-\mathrm{H} 91$ & 13.33 & LP (2) O87 & $R Y^{*}(4) \mathrm{H} 55$ & 0.08 \\
\hline $\mathrm{BD}^{*}(1) \mathrm{S} 165-\mathrm{O} 166$ & $\mathrm{BD}^{*}(1) \mathrm{N} 90-\mathrm{H} 91$ & 3.44 & LP (2) O87 & $\mathrm{BD}^{*}(1) \mathrm{N} 54-\mathrm{H} 55$ & 0.4 \\
\hline $\mathrm{BD}(1) \mathrm{N} 90-\mathrm{H} 91$ & $R Y^{*}(1) O 166$ & 0.27 & LP (3) O87 & $R Y^{*}(1) \mathrm{H} 55$ & 0.55 \\
\hline $\mathrm{BD}(1) \mathrm{N} 90$ - H91 & $\mathrm{BD}^{*}(1) \mathrm{S} 165-\mathrm{O} 166$ & 0.84 & LP (3) O87 & $R Y^{*}(6) \mathrm{H} 55$ & 0.08 \\
\hline & & & LP (3) O87 & $\mathrm{BD}^{*}(1) \mathrm{N} 54-\mathrm{H} 55$ & 1.08 \\
\hline & & & $\mathrm{BD}^{*}(1) \mathrm{O} 87$ - S 169 & $\mathrm{RY}^{*}(3) \mathrm{H} 55$ & 2.82 \\
\hline Total & & 32.07 & $\begin{array}{l}\mathrm{BD}^{*}(1) \mathrm{O} 087 \text { - S } 169 \\
\text { Total }\end{array}$ & $\mathrm{BD}^{*}(1) \mathrm{N} 54$ - H55 & $\begin{array}{r}5.06 \\
32.49 \\
\end{array}$ \\
\hline $4 \mathrm{BD}(1) \mathrm{C} 1$ - O25 & $R Y^{*}(1) \mathrm{H} 147$ & 0.49 & $\mathrm{BD}$ (1) N66 - H67 & $\mathrm{BD}^{*}(1) \mathrm{C} 170-\mathrm{O} 195$ & 0.63 \\
\hline $\mathrm{BD}(1) \mathrm{C} 1-\mathrm{O} 25$ & $\mathrm{RY}^{*}(2) \mathrm{H} 147$ & 0.14 & $\mathrm{BD}^{*}(1) \mathrm{N} 66-\mathrm{H} 67$ & $\mathrm{BD}^{*}(1) \mathrm{C} 170-\mathrm{O} 195$ & 1.11 \\
\hline $\mathrm{BD}(1) \mathrm{C} 1$ - O25 & $R Y^{*}(3) \mathrm{H} 147$ & 0.21 & BD (1) C 170 - O 195 & $R Y^{*}(5) \mathrm{H} 67$ & 0.07 \\
\hline $\mathrm{BD}(1) \mathrm{C} 1-\mathrm{O} 25$ & $\mathrm{BD}^{*}(1) \mathrm{N} 90-\mathrm{H} 147$ & 0.53 & BD (1) C 170 - O 195 & $\mathrm{BD}^{*}(1) \mathrm{N} 66-\mathrm{H} 67$ & 0.59 \\
\hline $\mathrm{BD}(2) \mathrm{C} 1-\mathrm{O} 25$ & $R Y^{*}(1) H 147$ & 0.1 & BD (1) C 170 - O 195 & $R Y^{*}(6) \mathrm{H} 67$ & 0.08 \\
\hline $\mathrm{BD}(2) \mathrm{C} 1-\mathrm{O} 25$ & $R Y^{*}(2) \mathrm{H} 147$ & 0.16 & CR (1) O 195 & $\mathrm{BD}^{*}(1) \mathrm{N} 66-\mathrm{H} 67$ & 0.48 \\
\hline
\end{tabular}




\begin{tabular}{|c|c|c|c|c|c|}
\hline $\mathrm{BD}(2) \mathrm{C} 1$ - O25 & $\mathrm{RY}^{*}(3) \mathrm{H} 147$ & 0.37 & LP (1) O 195 & $R Y^{*}(1) \mathrm{H} 67$ & 0.21 \\
\hline $\mathrm{BD}(2) \mathrm{C} 1-\mathrm{O} 25$ & $\mathrm{RY}^{*}(4) \mathrm{H} 147$ & 0.07 & LP (1) O 195 & $\mathrm{RY}^{*}(4) \mathrm{H} 67$ & 0.12 \\
\hline $\mathrm{BD}(2) \mathrm{C} 1-\mathrm{O} 25$ & $\mathrm{BD}^{*}(1) \mathrm{N} 90-\mathrm{H} 147$ & 0.77 & $\operatorname{LP}(1) O 195$ & $\mathrm{BD}^{*}(1) \mathrm{N} 66-\mathrm{H} 67$ & 6.81 \\
\hline CR (1) O25 & $\mathrm{BD}^{*}(1) \mathrm{N} 90-\mathrm{H} 147$ & 0.3 & LP (2) O 195 & $R Y^{\star}(2) \mathrm{H} 67$ & 1.46 \\
\hline LP (1) O25 & $R Y^{*}(2) \mathrm{H} 147$ & 0.96 & LP (2) O 195 & $\mathrm{RY}^{*}(4) \mathrm{H} 67$ & 0.35 \\
\hline LP (1) O25 & $R Y^{*}(3) \mathrm{H} 147$ & 0.08 & LP (2) O 195 & $\mathrm{RY}^{*}(7) \mathrm{H} 67$ & 0.05 \\
\hline LP (1) O25 & $\mathrm{BD}^{*}(1) \mathrm{N} 90-\mathrm{H} 147$ & 17.28 & LP (2) O 195 & $\mathrm{RY}^{\star}(8) \mathrm{H} 67$ & 0.06 \\
\hline LP (2) O25 & $R Y^{*}(2) H 147$ & 0.44 & LP (2) O 195 & $\mathrm{BD}^{*}(1) \mathrm{N} 66-\mathrm{H} 67$ & 31.56 \\
\hline LP (2) O25 & $\mathrm{BD}^{*}(1) \mathrm{N} 90-\mathrm{H} 147$ & 5.58 & $\mathrm{BD}^{*}(2) \mathrm{C} 170-\mathrm{O} 195$ & $\mathrm{BD}^{*}(1) \mathrm{N} 66-\mathrm{H} 67$ & 0.09 \\
\hline $\mathrm{BD}^{*}(2) \mathrm{C} 1-\mathrm{O} 25$ & $\mathrm{BD}^{*}$ (1) N90 - H 147 & 0.73 & & & \\
\hline BD (1) N90 - H 147 & $\mathrm{RY}^{*}(2) \mathrm{O} 25$ & 0.23 & & & \\
\hline $\mathrm{BD}(1) \mathrm{N} 90-\mathrm{H} 147$ & $R Y^{*}(4) \mathrm{O} 25$ & 0.1 & & & \\
\hline BD (1) N90 - H 147 & $\mathrm{BD}^{*}(1) \mathrm{C} 1-\mathrm{O} 25$ & 1.01 & & & \\
\hline $\begin{array}{l}\text { BD (1) N90 - H } 147 \\
\text { Total }\end{array}$ & $\mathrm{BD}^{*}(2) \mathrm{C} 1$ - O25 & $\begin{array}{r}0.72 \\
30.27\end{array}$ & Total & & 43.67 \\
\hline
\end{tabular}

Table S7. Topological analysis of the electron density of A conformer of $\alpha_{2} \beta_{2}-\mathbf{P} \cdot 10 \mathrm{CSA}(\mathbf{R})$ complex.

\begin{tabular}{|c|l|r|r|r|}
\hline Guest & \multicolumn{1}{|c|}{ Atoms } & $\begin{array}{c}\text { Electron } \\
\text { delocalization index }\end{array}$ & $\begin{array}{c}\text { Electron Dnsity } \\
(\boldsymbol{\rho}(\mathbf{r})) \text {, a.u. }\end{array}$ & $\begin{array}{c}\text { Laplacian } \\
\text { of Rho }\end{array}$ \\
\hline “Standing" & $\mathrm{O}_{138} \& \mathrm{H}_{103}$ & 3.934 & 0.06974 & 0.15993 \\
\hline & $\mathrm{O}_{167} \& \mathrm{H}_{53}$ & 4.277 & 0.04978 & 0.16467 \\
\hline & $\mathrm{O}_{166} \& \mathrm{H}_{91}$ & 3.915 & 0.04906 & 0.15993 \\
\hline & $\mathrm{O}_{25} \& \mathrm{H}_{147}$ & 4.013 & 0.05257 & 0.17475 \\
\hline “Lying” & $\mathrm{O}_{81} \& \mathrm{H}_{130}$ & 3.888 & 0.07823 & 0.14315 \\
\hline & $\mathrm{O}_{87} \& \mathrm{H}_{51}$ & 4.225 & 0.04127 & 0.15588 \\
\hline & $\mathrm{O}_{87} \& \mathrm{H}_{55}$ & 4.062 & 0.04127 & 0.15757 \\
\hline & $\mathrm{O}_{195} \& \mathrm{H}_{67}$ & 3.938 & 0.04127 & 0.14724 \\
\hline
\end{tabular}

\section{NMR calculations and analysis}

In addition, the ${ }^{13} \mathrm{C}$ and ${ }^{15} \mathrm{~N}$ NMR shielding tensors and chemical shifts, as well as $\Delta \sigma_{\max }$ between the corresponding chemical shifts of the main dominant conformer $\mathbf{A}$ of $\alpha_{2} \beta_{2}-\mathbf{P} \cdot 10 \mathrm{CSA}(\mathbf{R})$ were calculated (Tables S8-S10). The calculated ${ }^{13} \mathrm{C}$ and ${ }^{15} \mathrm{~N}$ NMR chemical shifts were found to deviate from the experimentally measured data, while the calculated and experimental $\Delta \sigma_{\max }$ values are in good agreement (Table S8), except the $\Delta \sigma_{\max }$ between carbons 2 and 13, 3 and 12, and nitrogens 22 and 24, which showed strong deviation (Table S8). The disagreement between these three $\Delta \sigma_{\max }$ values can be explained by the presence and influence of solvent molecules situated in a larger cavity. In contrast to the smaller cavity, where only one "lying" guest molecule can be placed, in the larger cavity in addition to the "standing" guest molecule several solvent molecules can be placed as well. However, the continuum solvent model (SND) used represents solvent as a continuous medium and does not count for individual effects of "explicit" molecules, such as H-bonds formation or charge transfer. We suppose that the deviation from the experiment is due to not accounting for the individual solvent molecules' effects in the larger porphyrin cavity. Between other $\Delta \sigma_{\max }$ calculated using theoretical and experimental data, a good correlation is observed, proving that the main calculated conformation corresponds to the 


\section{SUPPORTING INEORMATION}

dominant conformation presented in solution, which shows non-equivalency of the carbon and nitrogen atoms. To improve agreement with experimental measurements the conformer $\mathbf{A}$ of $\alpha_{2} \beta_{2}-\mathbf{P} \cdot 10 \mathrm{CSA}(\mathbf{R})$ with one and two additional acetonitrile molecules was modelled, and its ${ }^{13} \mathrm{C}$ and ${ }^{15} \mathrm{~N}$ NMR shielding tensors and chemical shifts, as well as $\Delta \sigma_{\max }$ between the corresponding chemical shifts were calculated. Inclusion of explicit solvent molecules in the model system improved agreement with the experimental data, especially in the case of the $\Delta \sigma_{\max }$ between carbons 2 and 13,3 and 12 , and nitrogens 22 and 24 (Table S8). However, the model system is sensitive to the presence of solvent molecules, especially in the case of peripheral atoms. Thus, to perform accurate NMR calculations, the whole solvent shell should be modelled which is beyond the scope of this study.

Table S8. Calculated shielding tensors and chemical shifts of ${ }^{13} \mathrm{C}$ of conformer $\mathbf{A}$ of $\alpha_{2} \beta_{2}-\mathbf{P} \cdot 10 \mathrm{CSA}(\mathbf{R})$ with and without solvent molecules.

\begin{tabular}{|c|c|c|c|c|c|c|}
\hline \multirow{2}{*}{$\begin{array}{l}\text { Number of } \\
\text { atom (in xyz) }\end{array}$} & \multicolumn{2}{|c|}{$\alpha_{2} \beta_{2}-\mathrm{P} \cdot 10 \mathrm{CSA}(\mathrm{R})$} & \multicolumn{2}{|c|}{$\begin{array}{c}\alpha_{2} \beta_{2}-P \cdot 10 \operatorname{CSA}(R)+ \\
1 \text { acetonitrile }\end{array}$} & \multicolumn{2}{|c|}{$\begin{array}{c}\alpha_{2} \beta_{2}-P \cdot 10 C S A(R)+ \\
2 \text { acetonitrile }\end{array}$} \\
\hline & $\begin{array}{c}\text { Shielding } \\
\text { tensor, ppm }\end{array}$ & $\begin{array}{c}\text { Scaled } \\
\text { chemical } \\
\text { shift, ppm }\end{array}$ & $\begin{array}{c}\text { Shielding } \\
\text { tensor, ppm }\end{array}$ & $\begin{array}{c}\text { Scaled } \\
\text { chemical } \\
\text { shift, ppm }\end{array}$ & $\begin{array}{l}\text { Shielding } \\
\text { tensor, ppm }\end{array}$ & $\begin{array}{c}\text { Scaled } \\
\text { chemical } \\
\text { shift, ppm }\end{array}$ \\
\hline 1 & $-60,94$ & 236,3 & $-62,83$ & 238,1 & $-62,84$ & 238,1 \\
\hline 2 & 116,32 & 63,0 & 115,74 & 63,5 & 115,62 & 63,7 \\
\hline 3 & 134,16 & 45,5 & 133,94 & 45,7 & 133,87 & 45,8 \\
\hline 4 & 132,32 & 47,3 & 132,12 & 47,5 & 132,25 & 47,4 \\
\hline 7 & 123,17 & 56,3 & 122,69 & 56,7 & 123,21 & 56,2 \\
\hline 8 & 151,34 & 28,7 & 151,37 & 28,7 & 151,04 & 29,0 \\
\hline 12 & 161,76 & 18,5 & 162,02 & 18,3 & 160,60 & 19,7 \\
\hline 16 & 160,08 & 20,2 & 159,39 & 20,9 & 160,69 & 19,6 \\
\hline 20 & 120,29 & 59,1 & 119,34 & 60,0 & 119,56 & 59,8 \\
\hline 22 & 151,16 & 28,9 & 151,74 & 28,3 & 151,49 & 28,6 \\
\hline 26 & 29,40 & 147,9 & 26,32 & 151,0 & 26,31 & 151,0 \\
\hline 27 & 25,99 & 151,3 & 27,38 & 149,9 & 28,09 & 149,2 \\
\hline 28 & 32,82 & 144,6 & 34,39 & 143,1 & 34,25 & 143,2 \\
\hline 29 & 31,22 & 146,2 & 29,68 & 147,7 & 27,94 & 149,4 \\
\hline 30 & 32,35 & 145,1 & 31,80 & 145,6 & 31,94 & 145,5 \\
\hline 31 & 58,80 & 119,2 & 58,84 & 119,2 & 59,68 & 118,3 \\
\hline 32 & 21,06 & 156,1 & 24,13 & 153,1 & 25,61 & 151,6 \\
\hline 33 & 19,13 & 158,0 & 24,03 & 153,2 & 24,98 & 152,3 \\
\hline 34 & 31,12 & 146,3 & 32,41 & 145,0 & 33,27 & 144,2 \\
\hline 35 & 56,16 & 121,8 & 57,43 & 120,5 & 57,68 & 120,3 \\
\hline 36 & 32,74 & 144,7 & 31,95 & 145,5 & 32,89 & 144,5 \\
\hline 37 & 25,41 & 151,9 & 27,73 & 149,6 & 28,54 & 148,8 \\
\hline 38 & 28,52 & 148,8 & 32,52 & 144,9 & 33,09 & 144,3 \\
\hline 39 & 34,09 & 143,4 & 32,57 & 144,8 & 32,93 & 144,5 \\
\hline 40 & 27,04 & 150,3 & 26,72 & 150,6 & 25,79 & 151,5 \\
\hline 41 & 32,40 & 145,0 & 30,66 & 146,7 & 27,61 & 149,7 \\
\hline 42 & 65,21 & 112,9 & 63,93 & 114,2 & 63,58 & 114,5 \\
\hline 43 & 63,20 & 114,9 & 62,58 & 115,5 & 63,69 & 114,4 \\
\hline 46 & 23,15 & 154,1 & 22,41 & 154,8 & 22,83 & 154,4 \\
\hline 47 & 156,80 & 23,4 & 156,72 & 23,5 & 157,14 & 23,1 \\
\hline 56 & 159,95 & 20,3 & 159,97 & 20,3 & 160,12 & 20,1 \\
\hline 60 & 44,40 & 133,3 & 43,94 & 133,7 & 43,38 & 134,3 \\
\hline
\end{tabular}




\begin{tabular}{|c|c|c|c|c|c|c|}
\hline 61 & 41,49 & 136,1 & 41,21 & 136,4 & 41,72 & 135,9 \\
\hline 62 & 49,57 & 128,2 & 50,24 & 127,6 & 50,81 & 127,0 \\
\hline 63 & 39,15 & 138,4 & 39,29 & 138,3 & 39,90 & 137,7 \\
\hline 64 & 34,31 & 143,1 & 34,90 & 142,6 & 33,32 & 144,1 \\
\hline 70 & 158,23 & 22,0 & 158,15 & 22,1 & 157,80 & 22,4 \\
\hline 74 & 160,38 & 19,9 & 160,20 & 20,1 & 159,79 & 20,5 \\
\hline 78 & 157,83 & 22,4 & 157,57 & 22,6 & 157,61 & 22,6 \\
\hline 82 & 156,63 & 23,6 & 156,30 & 23,9 & 156,46 & 23,7 \\
\hline 86 & 42,39 & 135,2 & 43,85 & 133,8 & 43,94 & 133,7 \\
\hline 88 & 35,22 & 142,3 & 32,81 & 144,6 & 32,98 & 144,4 \\
\hline 93 & 158,68 & 21,6 & 158,88 & 21,4 & 158,80 & 21,4 \\
\hline 95 & 156,68 & 23,5 & 156,86 & 23,3 & 156,81 & 23,4 \\
\hline 99 & 157,59 & 22,6 & 157,99 & 22,2 & 158,11 & 22,1 \\
\hline 104 & 154,57 & 25,6 & 156,19 & 24,0 & 156,17 & 24,0 \\
\hline 106 & 163,44 & 16,9 & 160,73 & 19,5 & 160,98 & 19,3 \\
\hline 110 & 157,01 & 23,2 & 157,18 & 23,0 & 157,48 & 22,7 \\
\hline 113 & 157,94 & 22,3 & 157,97 & 22,2 & 157,83 & 22,4 \\
\hline 117 & 42,89 & 134,8 & 42,18 & 135,5 & 42,12 & 135,5 \\
\hline 118 & 41,72 & 135,9 & 41,60 & 136,0 & 41,09 & 136,5 \\
\hline 119 & 49,74 & 128,1 & 50,10 & 127,7 & 50,04 & 127,8 \\
\hline 121 & 36,67 & 140,8 & 37,11 & 140,4 & 37,42 & 140,1 \\
\hline 123 & 41,87 & 135,8 & 42,22 & 135,4 & 42,65 & 135,0 \\
\hline 125 & 38,12 & 139,4 & 39,35 & 138,2 & 40,00 & 137,6 \\
\hline 131 & 162,15 & 18,2 & 161,69 & 18,6 & 161,30 & 19,0 \\
\hline 135 & 156,29 & 23,9 & 156,36 & 23,8 & 156,56 & 23,6 \\
\hline 139 & 32,97 & 144,5 & 31,01 & 146,4 & 31,17 & 146,2 \\
\hline 140 & 42,73 & 134,9 & 42,79 & 134,9 & 43,29 & 134,4 \\
\hline 141 & 48,71 & 129,1 & 48,55 & 129,2 & 48,55 & 129,2 \\
\hline 143 & 36,11 & 141,4 & 36,42 & 141,1 & 36,65 & 140,9 \\
\hline 145 & 40,72 & 136,9 & 41,06 & 136,5 & 41,14 & 136,5 \\
\hline 148 & 41,20 & 136,4 & 41,82 & 135,8 & 41,65 & 136,0 \\
\hline 149 & 43,16 & 134,5 & 43,54 & 134,1 & 43,62 & 134,0 \\
\hline 150 & 35,43 & 142,0 & 32,88 & 144,5 & 32,89 & 144,5 \\
\hline 151 & 51,34 & 126,5 & 50,74 & 127,1 & 51,00 & 126,8 \\
\hline 152 & 41,03 & 136,6 & 40,46 & 137,1 & 40,50 & 137,1 \\
\hline 154 & 37,23 & 140,3 & 37,08 & 140,4 & 37,42 & 140,1 \\
\hline 160 & 160,91 & 19,4 & 160,84 & 19,4 & 160,85 & 19,4 \\
\hline 170 & $-64,04$ & 239,3 & $-63,16$ & 238,4 & $-62,54$ & 237,8 \\
\hline 171 & 114,95 & 64,3 & 114,51 & 64,7 & 114,34 & 64,9 \\
\hline 172 & 134,30 & 45,4 & 132,65 & 47,0 & 133,12 & 46,5 \\
\hline 173 & 133,26 & 46,4 & 134,01 & 45,7 & 133,87 & 45,8 \\
\hline 176 & 121,90 & 57,5 & 122,19 & 57,2 & 122,56 & 56,9 \\
\hline 177 & 152,41 & 27,7 & 152,34 & 27,7 & 152,55 & 27,5 \\
\hline 181 & 162,30 & 18,0 & 161,69 & 18,6 & 161,27 & 19,0 \\
\hline 185 & 159,21 & 21,0 & 158,81 & 21,4 & 158,71 & 21,5 \\
\hline 189 & 121,29 & 58,1 & 120,40 & 59,0 & 120,18 & 59,2 \\
\hline 192 & 146,85 & 33,1 & 146,10 & 33,8 & 146,05 & 33,9 \\
\hline 196 & 36,05 & 141,4 & 36,61 & 140,9 & 36,86 & 140,7 \\
\hline 202 & & & 59,37 & 118,6 & 60,19 & 117,8 \\
\hline 203 & & & 180,29 & 0,4 & 179,90 & 0,8 \\
\hline 208 & & & & & 54,36 & 123,5 \\
\hline 209 & & & & & 180,40 & 0,3 \\
\hline
\end{tabular}


SUPPORTING INFORIMATION

Table S9. Calculated shielding tensors of ${ }^{15} \mathrm{~N}$ of conformer $\mathbf{A}$ of $\alpha_{2} \beta_{2}-\mathbf{P} \cdot 10 \mathrm{CSA}(\mathbf{R})$ with and without solvent molecules.

\begin{tabular}{|c|c|c|r|r|r|r|}
\hline \multirow{2}{*}{$\begin{array}{c}\text { Number of } \\
\text { atom } \\
\text { (in xyz) }\end{array}$} & \multicolumn{2}{|c|}{$\boldsymbol{\alpha}_{2} \boldsymbol{\beta}_{2}$-P·10CSA(R) } & \multicolumn{2}{c|}{$\begin{array}{c}\boldsymbol{\alpha}_{2} \boldsymbol{\beta}_{2} \text {-P·10CSA(R) + } \\
\mathbf{1} \text { acetonitrile }\end{array}$} & \multicolumn{2}{c|}{$\begin{array}{c}\boldsymbol{\alpha}_{2} \boldsymbol{\beta}_{2} \text {-P·10CSA(R) + } \\
\text { 2 acetonitrile }\end{array}$} \\
\cline { 2 - 7 } & $\begin{array}{c}\text { Shielding } \\
\text { tensor, ppm }\end{array}$ & $\begin{array}{c}\text { Scaled } \\
\text { chemical } \\
\text { shift, ppm }\end{array}$ & $\begin{array}{c}\text { Shielding } \\
\text { tensor, ppm }\end{array}$ & $\begin{array}{c}\text { Scaled } \\
\text { chemical } \\
\text { shift, ppm }\end{array}$ & $\begin{array}{c}\text { Shielding } \\
\text { tensor, ppm }\end{array}$ & $\begin{array}{c}\text { Scaled } \\
\text { chemical } \\
\text { shift, ppm }\end{array}$ \\
\hline 44 & 99,30 & 154,4 & 87,41 & 166,3 & 86,84 & 166,9 \\
\hline 50 & 99,33 & 154,4 & 90,15 & 163,5 & 91,46 & 162,2 \\
\hline 52 & 91,95 & 161,7 & 99,64 & 154,1 & 100,58 & 153,1 \\
\hline 54 & 106,38 & 147,3 & 101,99 & 151,7 & 101,91 & 151,8 \\
\hline 66 & 180,72 & 73 & 180,58 & 73,1 & 180,73 & 73 \\
\hline 90 & 185,44 & 68,3 & 184,27 & 69,4 & 184,17 & 69,5 \\
\hline 100 & 173,69 & 80 & 173,26 & 80,4 & 173,23 & 80,5 \\
\hline 127 & 178,41 & 75,3 & 177,34 & 76,4 & 176,61 & 77,1 \\
\hline & & & 6,94 & 246,8 & 2,86 & 250,8 \\
\hline & & & & & $-20,51$ & 274,2 \\
\hline
\end{tabular}

Table S10. Calculated and experimentally measured $\Delta \sigma_{\max }(\mathrm{ppm})$ between chemical shifts in ${ }^{13} \mathrm{C}$ of of $\alpha_{2} \beta_{2-}$ $\mathbf{P} \cdot 10 \mathrm{CSA}(\mathbf{R})$ with and without solvent molecules. Right side bottom, illustration of the $\alpha_{2} \beta_{2}-\mathbf{P}$ (blue - above and red - below the plane) with corresponding positions.

\begin{tabular}{|c|c|c|c|c|}
\hline \multirow[b]{2}{*}{ Positions } & \multicolumn{3}{|c|}{ Calculated } & \multirow[b]{2}{*}{ Exp. } \\
\hline & Conf. A & $\begin{array}{l}\text { Conf. A + } \\
1 \mathrm{CH}_{3} \mathrm{CN}\end{array}$ & $\begin{array}{l}\text { Conf. A + } \\
2 \mathrm{CH}_{3} \mathrm{CN}\end{array}$ & \\
\hline 1 and 14 & 1,7 & 0,0 & 0,0 & 0,98 \\
\hline 2 and 13 & 11,5 & 0,0 & 0,0 & 0,59 \\
\hline $2^{1}$ and $13^{1}$ & 1,2 & 0,0 & 0,0 & 0,26 \\
\hline $2^{2}$ and $13^{2}$ & 2,4 & 0,0 & 0,0 & 0,22 \\
\hline 3 and 12 & 6,7 & 0,0 & 0,0 & 0,22 \\
\hline $3^{1}$ and $12^{1}$ & 3,2 & 0,0 & 0,0 & 0,12 \\
\hline $3^{2}$ and $12^{2}$ & 6,7 & 0,0 & 0,0 & 0,12 \\
\hline 4 and 11 & 1,2 & 0,0 & 0,0 & 0,40 \\
\hline 6 and 9 & 1,3 & 0,0 & 0,0 & 0,35 \\
\hline 7 and 8 & 3,0 & 0,0 & 0,0 & 0,88 \\
\hline $7^{1}$ and $8^{1}$ & 1,1 & 0,0 & 0,0 & 0,19 \\
\hline $7^{2}$ and $8^{2}$ & 4,1 & 0,0 & 0,0 & 0,02 \\
\hline 10 and 5 & 2,6 & 0,0 & 0,0 & 0,47 \\
\hline $10^{1}$ and $5^{1}$ & 1,2 & 0,0 & 0,0 & 0,06 \\
\hline $10^{2}$ and $5^{2}$ & 0,4 & 0,0 & 0,0 & 0,09 \\
\hline $10^{3}$ and $5^{3}$ & 2,6 & 0,0 & 0,0 & 0,14 \\
\hline $10^{4}$ and $5^{4}$ & 1,1 & 0,0 & 0,0 & 0,00 \\
\hline $10^{5}$ and $5^{5}$ & 0,3 & 0,0 & 0,0 & 0,00 \\
\hline $10^{6}$ and $5^{6}$ & 0,2 & 0,0 & 0,0 & 0,04 \\
\hline 15 and 20 & 2,0 & 0,0 & 0,0 & 0,19 \\
\hline $15^{1}$ and $20^{1}$ & 1,5 & 0,0 & 0,0 & 0,00 \\
\hline $15^{2}$ and $20^{2}$ & 0,2 & 0,0 & 0,0 & 0,30 \\
\hline $15^{3}$ and $20^{3}$ & 0,2 & 0,0 & 0,0 & 0,00 \\
\hline $15^{4}$ and $20^{4}$ & 0,6 & 0,0 & 0,0 & 0,00 \\
\hline $15^{5}$ and $20^{5}$ & 2,7 & 0,0 & 0,0 & 0,00 \\
\hline $15^{6}$ and $20^{6}$ & 3,7 & 0,0 & 0,0 & 1,30 \\
\hline 16 and 19 & 2,9 & 0,0 & 0,0 & 0,52 \\
\hline 17 and 18 & 3,8 & 0,0 & 0,0 & 0,20 \\
\hline
\end{tabular}

\begin{tabular}{ll}
\hline $0-1.40 \mathrm{ppm}$ & $0.0-0.14 \mathrm{ppm}$ \\
\hline $1.50-2.9 \mathrm{ppm}$ & $0.15-0.30 \mathrm{ppm}$ \\
\hline $3.0-6.0 \mathrm{ppm}$ & $0.31-0.4 \mathrm{ppm}$ \\
\hline $6.0 \mathrm{ppm}$ & $>0.4 \mathrm{ppm}$ \\
\hline
\end{tabular}

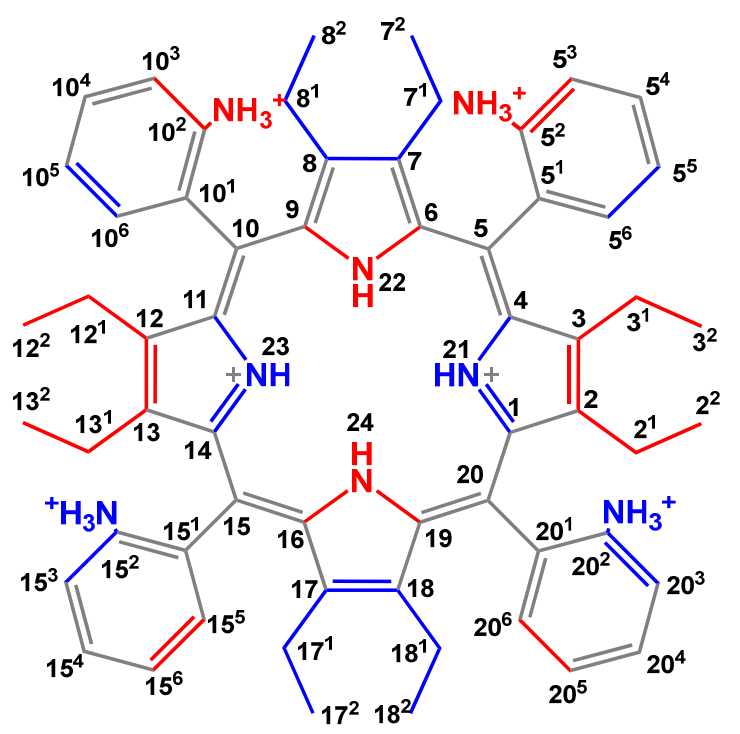




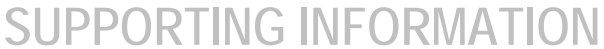

\begin{tabular}{|l|r|r|r|r|}
$17^{1}$ and $18^{1}$ & 0,5 & 0,0 & 0,0 & 0,12 \\
\hline $17^{2}$ and $18^{2}$ & 2,2 & 0,0 & 0,0 & 1,18 \\
\hline 21 and 23 & 7,0 & $-12,2$ & $-13,7$ & 1,67 \\
\hline 22 and 24 & 7,4 & $-11,8$ & $-10,4$ & 0,00 \\
\hline
\end{tabular}

\section{NBO partial charges calculations and analysis}

In order to clarify the source of $\Delta \sigma$ in ${ }^{13} \mathrm{C}$ and ${ }^{15} \mathrm{~N}$ NMR, the NBO partial charges were calculated for the following complexes: two porphyrin cations $4+$ (differing by the position of protons), porphyrin cation $6+$, optimized $\left[\alpha_{2} \beta_{2}-\mathrm{P}\left[\mathrm{H}_{2} \mathrm{SO}_{4}\right]_{2}\right]^{4+}$ and $\left[\alpha_{2} \beta_{2}-\mathrm{P}\left[\mathrm{H}_{2} \mathrm{SO}_{4} \cdot \mathrm{H}_{2} \mathrm{O}_{2}\right]^{4+}\right.$ complexes (corresponding to the crystal structure and achiral environment), and conformer $\mathbf{A}$ of $\alpha_{2} \beta_{2}-\mathbf{P} \cdot 10 \mathrm{CSA}(\mathbf{R})$ without inclusion of solvent molecules and with addition of one and two acetonitrile molecules (Figure S15 and Table S9). The presence or absence of protons change the $\mathrm{N}(21)-\mathrm{N}(24) \mathrm{NBO}$ partial charges in the porphyrin cations $4+$ by $0.019-0.043$, wherein in the porphyrin cation $6+$ where all $N(21)-N(24)$ are protonated the partial charges are very similar and differ only by 0.001 . In the achiral $\left[\alpha_{2} \beta_{2}-\mathrm{P}\left[\mathrm{H}_{2} \mathrm{SO}_{4}\right]_{2}\right]^{4+}$ complex one of oxygen atoms of each $\mathrm{H}_{2} \mathrm{SO}_{4}$ is situated symmetrically in respect to the core protons that result in the similar partial charges of $\mathrm{N}(21)-\mathrm{N}(24)$. However, addition of two water molecules leads to the difference by $0.008-0.015$ in the $\mathrm{N}(21)-\mathrm{N}(24) \mathrm{NBO}$ partial charges. This increased difference in partial charges can be explained by the non-symmetric position of oxygens of $\mathrm{H}_{2} \mathrm{SO}_{4}$ with respect to the porphyrin's core protons and as a result of the $\mathrm{H}$-bonds' formation of different strength. That is in good agreement with the observation that protonation or deprotonation has a stronger influence on the $N(21)-N(24) N B O$ partial charges, than the $\mathrm{H}$-bonds formation. In the more asymmetric $\alpha_{2} \beta_{2}-\mathbf{P} \cdot 10 \mathrm{CSA}(\mathbf{R})$ complex, the NBO partial charges split up to 0.056 for $\mathrm{N}(21)-\mathrm{N}(24)$ following the above noticed trend. Addition of acetonitrile molecules results in decrease of the difference between the NBO partial charges of N(21)$\mathrm{N}(24)$, herein the difference between the NBO partial charges of $\mathrm{N}(22)$ and $\mathrm{N}(24)$ is much more sensitive to the presence of solvent molecules in the cavity. This agrees well with our NMR calculations.

The ortho-carbons in the porphyrin cations $4+$ and $6+$ have similar partial charges (differing up to 0.006) (Figure S15 and Table S9). However, in the $\left[\alpha_{2} \beta_{2}-\mathrm{P}\left[\mathrm{H}_{2} \mathrm{SO}_{4}\right]_{2}\right]^{4+}$ complex, due to the small size of the guest molecule only one $\mathrm{H}$-bond (instead of two) can be formed between the $\mathrm{NH}_{3}{ }^{+}$groups of porphyrin and oxygens of each $\mathrm{H}_{2} \mathrm{SO}_{4}$ guest molecule. This leads to differences between the NBO partial charges of ortho-carbons of up to 0.021 for phenyls with the $\mathrm{H}$-bonded and non-bonded $\mathrm{NH}_{3}{ }^{+}$groups. Addition of two water molecules allows the $\mathrm{H}$-bond formation with all four $\mathrm{NH}_{3}{ }^{+}$groups of porphyrin, although these bonds are not equal; this is proved by difference in the NBO partial charges of orthocarbons of up to 0.12 between phenyls with the $\mathrm{H}$-bonded $\mathrm{NH}_{3}{ }^{+}$groups. In $\alpha_{2} \beta_{2}-\mathbf{P} \cdot 10 \mathrm{CSA}(\mathbf{R})$, the larger size of camphorsulfonic acid allows the $\mathrm{H}$-bond formation with all four $\mathrm{NH}_{3}{ }^{+}$groups of porphyrin; however non-equality of the formed bonds results in differences between the NBO partial charges of orthocarbons of up to 0.13 . Wherein, the tilt of phenyl rings varied less than $5.0^{\circ}$ in the porphyrin cation $6+$, 


\section{SUPPORTING INFORMATION}

$\left[\alpha_{2} \beta_{2}-\mathbf{P}\left[\mathrm{H}_{2} \mathrm{SO}_{4}\right]_{2}\right]^{4+},\left[\alpha_{2} \beta_{2}-\mathbf{P}\left[\mathrm{H}_{2} \mathrm{SO}_{4} \cdot \mathrm{H}_{2} \mathrm{O}_{2}\right]^{4+}\right.$ and $\alpha_{2} \beta_{2}-\mathbf{P} \cdot 10 \mathrm{CSA}(\mathbf{R})$ complexes, which allowed us to exclude this factor from consideration. Inclusion of acetonitrile molecules into the model also had a minor effect on ortho-carbons.

Summarizing, based on the six model complexes and their NBO partial charges it was shown that $\Delta \sigma$ values rely on the interactions with the chiral guests of certain size and chirality transfer effect.

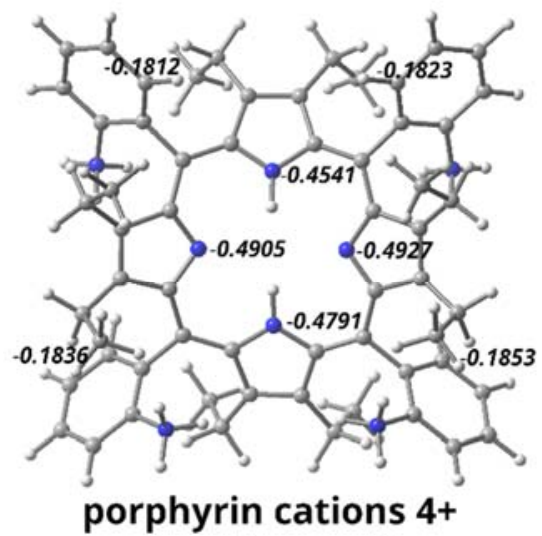

(conformer 1)

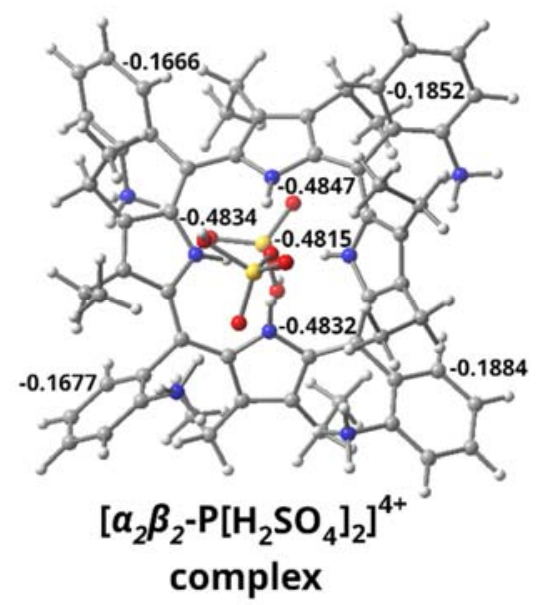

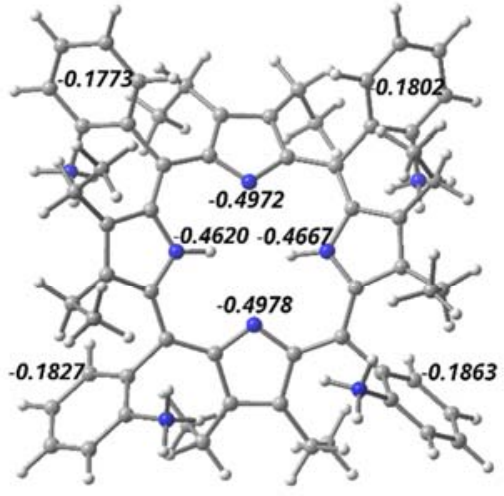

porphyrin cations 4+ (conformer 2)

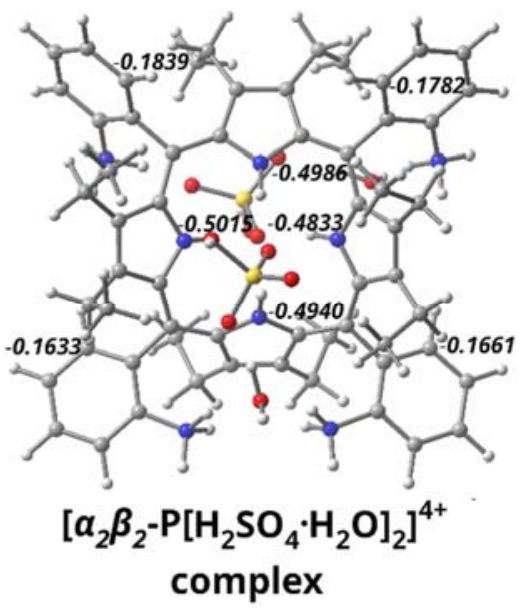

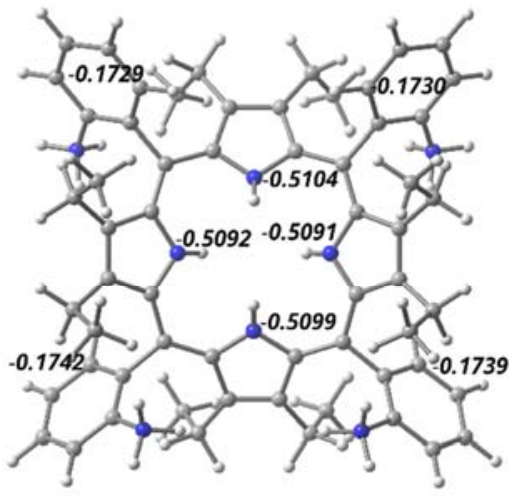

porphyrin cations $6+$

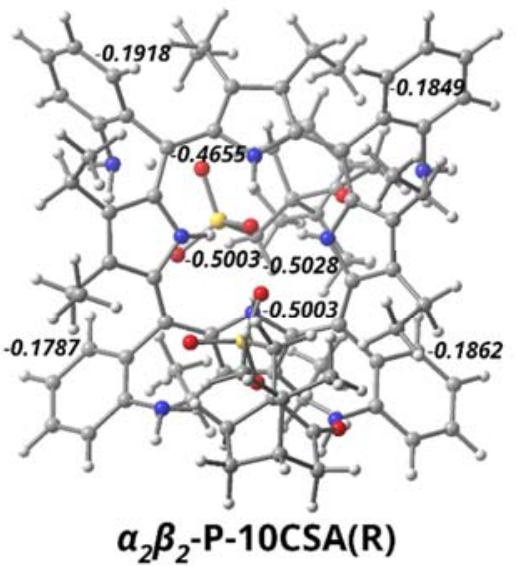

complex

Figure S15. NBO partial charges in variety of calculated complexes.

Table S9: Partial charges for core $\mathrm{N}$ atoms and ortho-carbons in different porphyrin complexes.

\begin{tabular}{|c|c|c|c|c|c|c|c|c|}
\hline $\begin{array}{l}\text { Atom } \\
\text { positions }\end{array}$ & $\begin{array}{l}{\left[\alpha_{2} \beta_{2}-\mathrm{P}\right]^{4+}} \\
\text { conf. } 1\end{array}$ & $\begin{array}{l}{\left[\alpha_{2} \beta_{2}-\mathrm{P}\right]^{4+}} \\
\text { conf. } 2\end{array}$ & {$\left[\alpha_{2} \beta_{2}-P\right]^{6+}$} & $\begin{array}{l}\boldsymbol{\alpha}_{2} \beta_{2^{-}} \\
\left.\mathrm{P}\left[\mathrm{H}_{2} \mathrm{SO}_{4}\right]_{2}\right]^{4+}\end{array}$ & $\begin{array}{l}\alpha_{2} \beta_{2^{-}} \\
\mathrm{P}\left[\mathrm{H}_{2} \mathrm{SO}_{4}\right. \\
\left.\left.\mathrm{H}_{2} \mathrm{O}\right]_{2}\right]^{4+}\end{array}$ & $\begin{array}{l}\alpha_{2} \beta_{2-} \\
P \cdot 10 \operatorname{CsA}(R)\end{array}$ & $\begin{array}{l}\alpha_{2} \beta_{2^{-}} \\
\mathrm{P} \cdot 10 \mathrm{CSA}(\mathrm{R}) \\
+ \\
1 \mathrm{CH}_{3} \mathrm{CN}\end{array}$ & $\begin{array}{l}\alpha_{2} \beta_{2^{-}} \\
\mathrm{P} \cdot 10 \mathrm{CSA}(\mathrm{R}) \\
+ \\
2 \mathrm{CH}_{3} \mathrm{CN} \\
\end{array}$ \\
\hline$N(22)$ & -0.47909 & -0.49783 & -0.50992 & -0.4832 & -0.49395 & -0.50025 & -0.49708 & -0.49635 \\
\hline$N(23)$ & -0.49272 & -0.46670 & -0.50905 & -0.4815 & -0.48329 & -0.50283 & -0.49102 & -0.49120 \\
\hline$N(24)$ & -0.45405 & -0.49720 & -0.51043 & -0.4847 & -0.49861 & -0.46549 & -0.49102 & -0.50509 \\
\hline$N(21)$ & -0.49051 & -0.46203 & -0.50923 & -0.4934 & -0.50147 & -0.52167 & -0.50010 & -0.49921 \\
\hline$C\left(5^{6}\right)$ & -0.18363 & -0.18273 & -0.17424 & -0.1677 & -0.16333 & -0.17866 & -0.17550 & -0.17476 \\
\hline$C\left(10^{6}\right)$ & -0.18532 & -0.18629 & -0.17385 & -0.1884 & -0.16609 & -0.18624 & -0.18431 & -0.18450 \\
\hline$C\left(15^{6}\right)$ & -0.18225 & -0.18021 & -0.17303 & -0.1852 & -0.17821 & -0.18491 & -0.18454 & -0.18664 \\
\hline$C\left(20^{6}\right)$ & -0.18124 & -0.17726 & -0.17285 & -0.1666 & -0.18393 & -0.19176 & -0.19280 & -0.19389 \\
\hline
\end{tabular}




\section{Geometries}

200

\section{Conformer A}

$\begin{array}{llll}\text { C } & 2.75945500 & 1.52518200 & 2.09538300\end{array}$ $\begin{array}{lll}-0.82229900 & -3.66595300 & 0.98781700\end{array}$ $0.13152300-3.55406300 \quad 2.01257500$ $0.97671200-2.41616700 \quad 1.68910400$ $\begin{array}{lll}-0.57190400 & -2.58876600 & 0.04321200\end{array}$ $-1.20924400-2.33173900-1.19933100$ $\begin{array}{lll}0.21127600 & 4.35461400 & 1.36816700\end{array}$ $\begin{array}{lll}-0.87750600 & 4.24667800 & 0.48976300\end{array}$ $\begin{array}{lll}-0.77574100 & 2.94962000 & -0.16584600\end{array}$ $\begin{array}{llll}-1.40952700 & 2.941428000 & -1.135647300\end{array}$ $\begin{array}{lll}-1.4092700 & 2.51428000 & -1.35643300\end{array}$ $\begin{array}{lll}-1.32782500 & 1.19718100 & -1.88417800\end{array}$ $\begin{array}{lll}-1.54335400 & 0.71584600 & -3.24495200\end{array}$ $\begin{array}{llll}-1.46252800 & -0.68573200 & -3.19904700\end{array}$

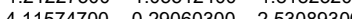
$\begin{array}{lll}4.1157400 & -0.29030300 & 2.53089300\end{array}$ $\begin{array}{lll}2.74912600 & -0.74379000 & 2.30880700 \\ 2.20733600 & -2.04960700 & 2.31363100\end{array}$ $\begin{array}{lll}2.23652300 & 2.81515500 & 1.85409900\end{array}$ $\begin{array}{lll}2.23652300 & 2.81515500 & 1.85409900 \\ 1.98710900 & 0.38066100 & 2.06528700\end{array}$ $\begin{array}{lll}1.98710900 & 0.38066170 & 2.06528700 \\ 0.96827900 & 0.37495700 & 1.97543800\end{array}$ $\begin{array}{lll}0.96827900 & 0.37495700 & 1.97543800 \\ 4.12689800 & 1.10208500 & 2.40249300\end{array}$ $\begin{array}{lll}4.12689800 & 1.10208500 & 2.40249300 \\ 53445900 & -1.13481900 & 2.73792800\end{array}$ $\begin{array}{lll}5.34459000 & -1.13481900 & 2.73792800 \\ 5.19344000 & -1.84769600 & 3.56898000\end{array}$ $\begin{array}{llll}5.19344000 & -1.84769600 & 3.56898000 \\ 6.17011300 & -0.47335500 & 3.06457700\end{array}$ $\begin{array}{lll}6.17011300 & -0.47335500 & 3.06457700 \\ 0.26145900 & 2.25515000 & 0.44418700\end{array}$ $\begin{array}{llll}0.73851700 & 1.43526300 & -0.01499700\end{array}$ $\begin{array}{llll}-1.06563400 & 0.10491600 & -1.09061200\end{array}$ $\begin{array}{lll}-1.28930700 & 0.11148900 & -0.06139700\end{array}$ $0.48121300-1.83235100 \quad 0.53880100$ $\begin{array}{lll}1.03912500 & -1.16023800 & -0.04261900\end{array}$ $\begin{array}{lll}5.79442700 & -1.90359700 & 1.48641900\end{array}$ $\begin{array}{llll}6.04199000 & -1.21050100 & 0.65920600 \\ 4.9998280 & -258368900 & 1.12494400\end{array}$ $\begin{array}{lll}6.69325400 & -2.51275100 & 1.70493400\end{array}$ $\begin{array}{llll}2.96219300 & -3.15598000 & 2.95382900\end{array}$ $\begin{array}{lll}3.9621559000 & -3.31232400 & 2.21791500\end{array}$ $\begin{array}{llll}4.01217900 & -5.37623300 & 2.79951100\end{array}$ $\begin{array}{lll}4.01217900 & -5.37623300 & 2.7951100 \\ 4.01283000 & -4.17791000 & 4.91714800\end{array}$ $\begin{array}{llll}4.01282000 & -3.1723500 & 2.79551100 \\ 3.04734900 & -2.22943600 & 431686200\end{array}$ $\begin{array}{llll}4.32720100 & -3.11256500 & 4.31680200\end{array}$ $\begin{array}{lll}3.04734900-2.22943600 & 4.91037400\end{array}$ $\begin{array}{llll}2.90773500 & -4.42533400 & 0.80135500 \\ 3.10552200 & -3.54296100 & 0.17013800\end{array}$ $\begin{array}{llll}1.87615200 & -4.57451300 & 0.74168000\end{array}$ $\begin{array}{llll}1.87615200 & -4.57451300 & 0.74138000 \\ 3.36715500 & -524156400 & 0.36268500\end{array}$ $\begin{array}{lll}1.311025900 & -4.30094300 & 3.32133400 \\ 0.11300\end{array}$ $\begin{array}{lll}0.11025900 & -4.30094300 & 3.32133400 \\ 1.09049200 & -4.76993700 & 3.53749000\end{array}$ $\begin{array}{llll}-0.60691600 & -5.14109000 & 3.23905900\end{array}$ $\begin{array}{lll}1.77718700 & 2.19396300 & -2.28218500\end{array}$ $\begin{array}{llll}-0.29138900 & -3.39458800 & 4.49838100\end{array}$ $0.43596400-2.57146500 \quad 4.64882500$ $\begin{array}{lll}-0.34015900 & -3.97796900 & 5.43867700\end{array}$ $\begin{array}{lll}-1.28558000 & -2.93346400 & 4.33007100\end{array}$ $\begin{array}{llll}-2.02693900 & -4.56377900 & 1.01384400\end{array}$ $\begin{array}{llll}-2.19059200 & -5.03693100 & 0.02829400\end{array}$ $\begin{array}{lll}-1.84564500 & -5.39840100 & 1.71891500\end{array}$ $\begin{array}{llll}3.78244700 & 1.66819700 & -0.89176100\end{array}$ $\begin{array}{lll}-3.28992600 & -3.79638000 & 1.44076100\end{array}$ $\begin{array}{lll}-3.19527500 & -3.42316300 & 2.48084900\end{array}$ $-4.18592200 \quad-4.44508700 \quad 1.39036400$ $-3.46412000 \quad-2.91662700 \quad 0.79186500$ $-1.87893300 \quad-3.47008600 \quad-1.88444900$ $\begin{array}{lll}-1.8789380 & -3.4708600 & -1.88444900\end{array}$ $-1.11391500-4.58715500-2.26400000$ $\begin{array}{llll}1.1 .11391500 & -4.59715500 & -2.26409600\end{array}$ $-0.03450200-4.3613500-2.05288700$ $\begin{array}{lll}-4.13202400 & -2.36927800 & -1.00709300\end{array}$ $-4.08108000-1512281000-1.35073800$ $-3.68108800-1.51228100-1.35973800$ $\begin{array}{lll}-1.38850700 & -1.60800600 & -1.358391200 \\ -1.77735400 & -1.0851000 & -5.27998700\end{array}$ $\begin{array}{rrr}-1.77736400 & -1.08651060 & -5.27996700 \\ 0.05357600 & -2.07879600 & -4.65188600\end{array}$

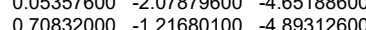
$\begin{array}{llll}0.70832000 & -1.21680100 & -4.659312600 \\ 0.08784100 & -2.77927100 & -5.50930500\end{array}$ $\begin{array}{llll}0.08784100 & -2.77927100 & -5.50930500 \\ 0.47921600 & -259941400 & -3.76947700\end{array}$ $\begin{array}{rrrr}0.47921600 & -2.59941400 & -3.76947700 \\ -161144400 & 152996200 & -45066800\end{array}$ $\begin{array}{llll}-1.61144400 & 1.52996200 & -4.50669800\end{array}$ $-4.31064900 \quad 2.52149700-1.61484800$ $\begin{array}{llll}-4.08412700 & 1.52228400 & -1.83700400\end{array}$ $\begin{array}{llll}-5.32182400 & 2.65592300 & -1.77610000\end{array}$ $\begin{array}{llll}-4.14087300 & 2.48952400 & -0.52504100\end{array}$ $\begin{array}{llll}-2.00176900 & 5.24261300 & 0.41033500\end{array}$ $\begin{array}{lll}-1.57506900 & 6.26735900 & 0.38997800\end{array}$ $\begin{array}{lll}-2.98469500 & 5.11098600 & 1.59182200\end{array}$ $\begin{array}{lll}-2.49737900 & 5.33812000 & 2.55995400\end{array}$ $\begin{array}{lll}-3.39768200 & 4.08522500 & 1.65562000\end{array}$ $\begin{array}{llll}-3.82300200 & 5.82510300 & 1.46784100\end{array}$ $\begin{array}{lll}0.43502300 & 5.49124400 & 2.32766300\end{array}$ $\begin{array}{lll}-0.22790700 & 6.33019900 & 2.03817900\end{array}$ $\begin{array}{lll}1.46633200 & 5.88404600 & 2.23424700\end{array}$ $\begin{array}{lll}1.46633200 & 5.88404600 & 2.23424700 \\ 0.16130400 & 5.10488200 & 3.79234100\end{array}$ $0.79215400 \quad 4.25549500 \quad 4.12081000$ $\begin{array}{rrr}0.79215400 & 4.25549500 & 4.12081000 \\ -0.89422000 & 4.80211400 & 3.94065100\end{array}$ $\begin{array}{lll}0.36752500 & 5.96173100 & 4.46355600\end{array}$ $\begin{array}{llll}3.11688700 & 4.00250300 & 2.06725000\end{array}$ $\begin{array}{llll}3.63432500 & 4.69054800 & 0.94661600 \\ 4.46100200 & 5.81152200 & 1.09159800\end{array}$ $\begin{array}{lll}4.86285200 & 6.32902100 & 0.20642000\end{array}$ $\begin{array}{llll}4.77410700 & 6.27394400 & 2.37973800\end{array}$ $\begin{array}{llll}5.41656800 & 7.15881200 & 2.49643300\end{array}$ $\begin{array}{llll}4.27131000 & 5.60567000 & 3.50858100\end{array}$ $\begin{array}{llll}4.51923800 & 5.96436700 & 4.51824500\end{array}$ $\begin{array}{llll}3.45850800 & 4.47421000 & 3.35118700\end{array}$ $\begin{array}{llll}3.07674500 & 3.94040400 & 4.23327600\end{array}$ $\begin{array}{llll}3.32405600 & 4.19849900 & -0.40489100\end{array}$ $\begin{array}{llll}3.32405600 & 4.19840900 & -0.40489100\end{array}$ $\begin{array}{lll}3.8267000 & 4.74688600 & -1.12262000\end{array}$ $\begin{array}{llll}2.31270000 & 4.26355300 & -0.63331600\end{array}$ $\begin{array}{llll}6.20690800 & 2.02517100 & -1.21774500\end{array}$

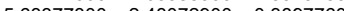
$\begin{array}{llll}5.63377800 & 2.48072900 & 0.38917600\end{array}$ $\begin{array}{llll}6.53726500 & 1.0259600 & 0.87029800\end{array}$ $\begin{array}{llll}5.37865100 & 1.93238700 & 2.51081100\end{array}$ $\begin{array}{lll}6.00840200 & 1.48493800 & 3.30736500 \\ 5.14541000 & 2.94750400 & 2.86774400\end{array}$ $\begin{array}{lll}-3.96100900 & -0.05509600 & -0.58739200\end{array}$ $\begin{array}{llll}0.94982200 & 3.10350000 & -1.29767600\end{array}$ $\begin{array}{lll}-3.26387600 & -3.49297500 & -2.16954500\end{array}$ $\begin{array}{llll}-3.86277900 & -4.59679300 & -2.79182100\end{array}$ $\begin{array}{lll}-4.94667100 & -4.60037000 & -2.98461900\end{array}$ $\begin{array}{llll}-3.07886100 & -5.69822400 & -3.16620600\end{array}$ $\begin{array}{lll}-3.55137500 & -6.55934600 & -3.66069300\end{array}$ $\begin{array}{llll}-1.69799600 & -5.69528400 & -2.90676000\end{array}$ $\begin{array}{lll}-1.07929900 & -6.55405500 & -3.20519700\end{array}$ $\begin{array}{lll}-4.64446000 & -2.01809800 & -2.63380500\end{array}$ $\begin{array}{lll}-2.11686900 & 3.53285600 & -2.18378600\end{array}$ $\begin{array}{llll}-3.51937300 & 3.51394100 & -2.34630000\end{array}$ $\begin{array}{llll}-1.38181400 & 4.53381200 & -2.85085200\end{array}$ $\begin{array}{llll}-4.17396900 & 4.45850500 & -3.14528700\end{array}$ $-202565300-5.4775$ $\begin{array}{llll}-2.02565300 & 5.47755900 & -3.66355200 \\ -0.2888500 & 4.54667400 & -2.73460500\end{array}$ $-3.28145100 \quad 5.44018900-3.73460500$ $\begin{array}{llll}-5.42146100 & 5.54018900 & -3.73455500\end{array}$ $\begin{array}{llll}-5.27005900 & 4.43883400 & -3.25053600\end{array}$ $\begin{array}{lll}-1.43618500 & 0.24723400 & -4.48433800\end{array}$

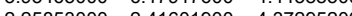

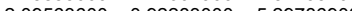
$\begin{array}{llll}-2.09563600 & 0.92283000 & -5.29786900\end{array}$ $\begin{array}{llll}-0.21714000 & 1.97868700 & -4.298425200\end{array}$ $\begin{array}{llll}0.40480400 & 1.10490400 & -5.26697900\end{array}$ $\begin{array}{llll}0.30409400 & 2.63088900 & -5.87531300\end{array}$ $\begin{array}{llll}0.33146400 & 2.52296900 & -4.19194600\end{array}$ $\begin{array}{llll}-2.55556700 & 5.13559500 & -4.53693000\end{array}$ $\begin{array}{lll}-3.64090700 & 0.43175500 & 0.83469300\end{array}$ $\begin{array}{lll}-2.26399200 & 0.02661900 & 1.29510200\end{array}$ $\begin{array}{lll}-5.96296800 & -2.33404700 & 0.10513600\end{array}$ $\begin{array}{lll}-2.03642200 & -2.49591600 & -4.24257000\end{array}$ $\begin{array}{llll}2.56756200 & 1.10444900 & -1.62910600\end{array}$ $\begin{array}{llll}4.06135300 & -2.17071500 & -1.68019100\end{array}$ $\begin{array}{llll}4.29981000 & -0.98709500 & -2.60476500\end{array}$ $\begin{array}{llll}5.97805600 & -2.49281800 & -3.07035500\end{array}$ $\begin{array}{lll}5.16018800 & -3.18276200 & -1.96215700\end{array}$ $5.74048100 \quad-3.41322800-1.04369600$ $\begin{array}{llll}4.69633700 & -4.13386700 & -2.29878200\end{array}$ $\begin{array}{lll}4.86628600 & -1.75124700 & -3.88689400\end{array}$ $\begin{array}{llll}6.74650000 & -1.33835900 & -2.39195300\end{array}$ $7.48618000-0.88262700-3.07637300$ $7.31046700-1.68897500-1.50513900$ $6.60337400-3.18541100-3.66390900$ $\begin{array}{llll}5.41879400 & -0.81564400 & -4.96942100\end{array}$ $\begin{array}{llll}5.46262600 & -0.288812200 & -5.59401600\end{array}$ $\begin{array}{llll}4.602365900 & -0.05163200 & -4.59215100\end{array}$ $\begin{array}{lll}6.12365900 & -0.05163200 & -4.59215100 \\ 5.96012800 & -1.41278900 & -5.73145400\end{array}$ $\begin{array}{llll}5.82380200 & -2.67857100 & -4.53435700\end{array}$ $\begin{array}{lll}3.82380200 & -2.67857100 & -4.53435700 \\ 3.07792800 & -2.09246200 & -5.10796000\end{array}$ $\begin{array}{llll}4.31031600 & -3.36703100 & -5.25433300\end{array}$ $\begin{array}{lll}4.31031600 & -3.36703100 & -5.25433300 \\ 3.25899500 & -3.30289100 & -3.81208300\end{array}$ $3.16586200-0.02522800-292076000$ $2.24504000-0.54593900-3.25221000$ $3.47993600 \quad 0.66655100 \quad-3.72812300$ $\begin{array}{llll}5.61547000 & -0.33992000 & -2.02416700\end{array}$ $\begin{array}{llll}5.74539800 & 0.65014300 & -2.50212300\end{array}$ $\begin{array}{llll}5.51638900 & -0.13398500 & -0.94302700\end{array}$ $3.15593400-2.31216700-0.84249000$ $\begin{array}{llll}4.36272400 & -5.30691900 & 4.15835100\end{array}$ $\begin{array}{llll}4.27594900 & -4.12763100 & 5.98383900\end{array}$ $\begin{array}{lll}4.28012400 & -6.26512400 & 2.20704500\end{array}$ $\begin{array}{lll}4.90535100 & -6.14398800 & 4.62111800\end{array}$ $\begin{array}{lll}-6.68197400 & -1.60361600 & 0.78769300\end{array}$ $\begin{array}{lll}-6.24649800 & -0.40864200 & 1.63803300\end{array}$ $\begin{array}{llll}-8.18273800 & -1.74917600 & 0.96805100\end{array}$ $\begin{array}{lll}-7.23695100 & -0.57683600 & 2.86966700\end{array}$ $\begin{array}{lll}-6.86416900 & 0.83014200 & 0.89590400\end{array}$ $\begin{array}{llll}-8.50872900 & -0.60819300 & 1.95313300\end{array}$ $\begin{array}{lll}-8.50872900 & -0.60819300 & 1.95313300 \\ -8.70719900 & -1.68551600 & -0.00853300\end{array}$ $\begin{array}{lll}-8.39945700 & -2.76317000 & 1.36424500\end{array}$ $\begin{array}{rrr}-7.20488800 & 0.60134600 & 3.85089600\end{array}$ $\begin{array}{lll}-6.98318200 & -1.87266400 & 3.65933900\end{array}$ $\begin{array}{lll}-6.42730400 & 1.75353400 & 1.32284300\end{array}$ $\begin{array}{lll}-6.59905400 & 0.81237300 & -0.18058400\end{array}$ $\begin{array}{lll}-8.39438900 & 0.71210200 & 1.15755000\end{array}$ $\begin{array}{lll}-9.46408200 & -0.73864400 & 2.49535000\end{array}$ $\begin{array}{lll}-8.77145400 & 1.57487500 & 1.73826400\end{array}$ $\begin{array}{llll}-8.98260900 & 0.67586700 & 0.22026900\end{array}$ $\begin{array}{lll}-4.77560400 & -0.35660000 & 2.00824400\end{array}$ $\begin{array}{lll}-4.34729100 & -1.36627300 & 2.15733100\end{array}$ $\begin{array}{lll}-4.60679600 & 0.23223500 & 2.93301500\end{array}$ $\begin{array}{lll}-3.88528000 & 1.93339000 & 0.89463900\end{array}$ $\begin{array}{llll}-7.31011300 & 1.59367200 \quad 3.37395400\end{array}$ $\begin{array}{lll}-7.31011300 & 1.59367200 & 3.37395400 \\ -6.26488600 & 0.61054200 & 4.44084900\end{array}$ $\begin{array}{llll}-6.26488600 & 0.61054200 & 4.44084900\end{array}$ $\begin{array}{lll}-8.03347800 & 0.49846500 & 4.58122900 \\ -6.05529000 & -1.79182400 & 4.26234600\end{array}$ $\begin{array}{ll}-6.058281000 & -1.77907200 \quad 3.26234600\end{array}$ $\begin{array}{lll}-7.81191100 & -2.05882700 & 4.37208900\end{array}$

200

\section{Conformer B}

\begin{tabular}{|c|c|c|c|}
\hline 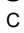 & 658600 & -1.79258000 & -1.34 \\
\hline C & -5.84619100 & -0.66271400 & -2.28257800 \\
\hline C & -8.12674400 & -0.54648400 & -2.14369900 \\
\hline C & -7.76448100 & -1.70914800 & -1.19233200 \\
\hline $\mathrm{H}$ & -8.23724700 & -2.68268700 & -1.43794900 \\
\hline & -8.01808900 & -1.50286100 & -0.13099600 \\
\hline C & -6.92248400 & 0.43956200 & -1.94125100 \\
\hline & -7.92090700 & -1.06813500 & -3.58165400 \\
\hline & -8.30706700 & -0.35442500 & -4.33352800 \\
\hline & -8.44536800 & -2.02845200 & -3.75077200 \\
\hline & -9.11985300 & -0.09965200 & -1.94877000 \\
\hline & -6.90532100 & 1.63299200 & -2.90372500 \\
\hline & -5.98244900 & 2.23257900 & -2.76355800 \\
\hline & -6.96500900 & 1.35113400 & -3.97147400 \\
\hline & -7.76515900 & 2.30191200 & -2.69401800 \\
\hline C & -6.85033600 & 0.98421800 & -0.50003000 \\
\hline & -6.10678900 & 1.80081300 & -0.45555400 \\
\hline & -7.83321500 & 1.40439300 & -0.20511100 \\
\hline & -6.56855800 & 0.21627200 & 0.24851300 \\
\hline & -4.36033000 & -0.37393600 & -2.39646500 \\
\hline & -4.14726900 & 0.37230800 & -3.18824100 \\
\hline C & -6.37672200 & -1.21178600 & -3.67341200 \\
\hline & -5.94172500 & -0.59406800 & -4.48451200 \\
\hline & -6.03392500 & -2.25135000 & -3.84802800 \\
\hline & -5.50643700 & -2.64742600 & -0.87302800 \\
\hline & 2.94090500 & -0.27851200 & -2.23029900 \\
\hline & -1.18758700 & 4.13720600 & -0.03326100 \\
\hline & -0.18369000 & 4.39839400 & -0.97862700 \\
\hline & 0.69527800 & 3.24116100 & -1.00957500 \\
\hline & -0.91554200 & 2.81617800 & 0.52836800 \\
\hline & -1.50140900 & 2.19077000 & 1.66067000 \\
\hline & 0.60076700 & -3.31986500 & -2.35451000 \\
\hline & -0.38760900 & -3.61960800 & -1.40329800 \\
\hline & -0.27403800 & -2.63777400 & -0.33227600 \\
\hline & -1.00957000 & -2.57737000 & 0.88556800 \\
\hline & -1.15209400 & -1.39955100 & 1.66325200 \\
\hline C & -1.57255500 & -1.22785000 & 3.04535000 \\
\hline & -1.68008800 & 0.15319900 & 3.27510500 \\
\hline C & -1.35544600 & 0.82344000 & 2.01747700 \\
\hline & 4.10305100 & 1.70449900 & -2.07672900 \\
\hline C & 2.67866400 & 1.95323600 & -1.87543800 \\
\hline & 1.98791900 & 3.15683800 & -1.60579800 \\
\hline & 2.54052000 & -1.62522200 & -2.39595800 \\
\hline & 2.03975900 & 0.73345900 & -1.96401300 \\
\hline & 1.02313100 & 0.61608700 & -1.98680100 \\
\hline & 4.25997000 & 0.33051800 & -2.30119100 \\
\hline & 5.25807500 & 2.66269100 & -1.94649900 \\
\hline & 4.92971900 & 3.70046300 & -2.11120200 \\
\hline & 5.97260200 & 2.44982700 & -2.76975900 \\
\hline & 0.73988200 & -1.75559900 & -0.68310900 \\
\hline & 1.20203500 & -1.08579500 & -0.02225700 \\
\hline & -0.97718900 & -0.14597400 & 1.11702500 \\
\hline & -1.11010900 & -0.00955600 & 0.08261900 \\
\hline & 0.16612300 & 2.28598000 & -0.15898200 \\
\hline & 0.74041800 & 1.47603500 & 0.20792900 \\
\hline & 5.99405500 & 2.57667100 & -0.59616800 \\
\hline & 6.43538500 & 1.57356500 & -0.42977700 \\
\hline & 5.30717100 & 2.78623900 & 0.24525900 \\
\hline & 6.82526800 & 3.30866100 & -0.57001100 \\
\hline & 2.69051800 & 4.45119400 & -1.81165100 \\
\hline & 2.99149400 & 5.27964500 & -0.70422800 \\
\hline & 3.67364900 & 6.49111000 & -0.86231400 \\
\hline & 3.74747200 & 6.11010200 & -3.26560300 \\
\hline & 3.08358400 & 4.88748500 & -3.09430300 \\
\hline & 2.86012100 & 4.25128800 & -3.96317500 \\
\hline & 2.56769700 & 4.86560300 & 0.64499600 \\
\hline & 2.78307800 & 3.78976500 & 0.87187700 \\
\hline & 1.54160600 & 4.99230700 & 0.75919600 \\
\hline & 3.03319100 & 5.43944400 & 1.36811600 \\
\hline & -0.15707500 & 5.57801400 & -1.91573600 \\
\hline & 0.81179000 & 6.11220500 & -1.86315500 \\
\hline & -0.91594 & 6.31137900 & -1.580388 \\
\hline
\end{tabular}


SUPPORTING INFORMATION

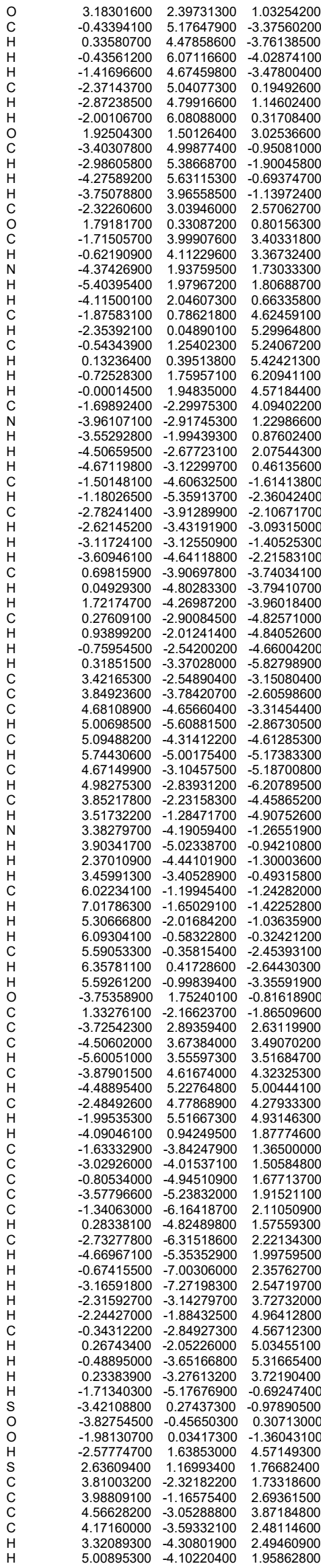

$\begin{array}{lll}5.24836300 & -1.68850100 & 3.51571100\end{array}$ $3.25455100-2.61681500 \quad 4.56004400$ $\begin{array}{lll}3.42026300 & -2.35829000 & 5.62257300 \\ 2.50020300 & -3.42743400 & 4.54660500\end{array}$ $\begin{array}{lll}2.50020300 & -3.42743400 & 4.54660500 \\ 5.19291000 & -3.74767100 & 4.46121900\end{array}$ $\begin{array}{lll}5.19291000 & -3.74767100 & 4.46121900 \\ 5.57971800 & -0.82222400 & 4.73674200\end{array}$ $\begin{array}{llll}5.57971800 & -0.82222400 & 4.73674200\end{array}$ $\begin{array}{lll}6.04137600 & 0.13973400 & 4.43362500\end{array}$ $\begin{array}{lll}4.70905100 & -0.58715700 & 5.37665800\end{array}$ $\begin{array}{lll}6.32537800 & -1.34442000 & 5.37044400\end{array}$ $\begin{array}{lll}6.51323400 & -1.80917300 & 2.64872500\end{array}$ $\begin{array}{lll}6.90584200 & -0.80607900 & 2.38416300 \\ 7.31679800 & -2.33002700 & 3.20737600\end{array}$ $\begin{array}{lll}7.31679800 & -2.33002700 & 3.20737600 \\ 6.36603000 & -2.36018200 & 1.69772200\end{array}$ $\begin{array}{lll}4.14688000 & 0.21591500 & 2.08512100\end{array}$ $\begin{array}{lll}4.65741900 & 0.18655700 & 1.10309200\end{array}$ $4.71923000 \quad 0.88201100 \quad 2.76136000$ $2.82062300-1.37518900 \quad 3.73082900$ $2.72922600-0.45423200-4.33760800$ $\begin{array}{lll}2.72922600 & -0.45423200 & 4.33760800 \\ 1.84799300 & -1.49255700 & 3.21369300\end{array}$ $3.45013300-2.24554100 \quad 0.54772200$ $\begin{array}{lll}3.45013300 & -2.24554100 & 0.54772200 \\ 4.05034700 & 6.90819900 & -2.1500000\end{array}$ $4.03781100 \quad 6.43870900-2.15000800$ $\begin{array}{lll}4.03781100 & 6.43870900 & -4.27419900 \\ 3.11770000 & 7.11800600 & 0.01119500\end{array}$ $4.91170000-7.86280100-2.27585100$ $\begin{array}{rrr}-3.80639000 & -1.30076700 & -2.64658000\end{array}$

200

\section{Conformer C}

C $\quad-2.74727900 \quad 1.60768600-2.03318300$ $\begin{array}{lll}0.69464400 & -3.69427300 & -0.99021800\end{array}$ $\begin{array}{llll}-0.25179800 & -3.53994400 & -2.01574800\end{array}$ $-1.06739500-2.38432000-1.67703500$ $\begin{array}{lll}0.47050200 & -2.62270600 & -0.03248200\end{array}$ $\begin{array}{lll}1.12973900 & -2.37636500 & 1.20021500\end{array}$ $\begin{array}{llll}-0.12351600 & 4.37822400 & -1.34704600\end{array}$ $\begin{array}{llll}0.97734900 & 4.25178900 & -0.48607300\end{array}$ $\begin{array}{lll}0.86125000 & 2.95686300 & 0.17274800\end{array}$ $\begin{array}{lll}1.53724400 & 2.48814800 & 1.32383600\end{array}$ $\begin{array}{lll}1.45106000 & 1.16206800 & 1.82941100\end{array}$ $\begin{array}{lll}1.67143300 & 0.67809500 & 3.18732800\end{array}$ $\begin{array}{lll}1.50080400 & -0.71747200 & 3.16076000\end{array}$ $\begin{array}{lll}1.50080400 & -0.71747200 & 3.16076000 \\ 1.21433900 & -1.08862800 & 1.78945800\end{array}$ $\begin{array}{lll}-4.15257800 & -0.16862600 & -2.47877300\end{array}$ $\begin{array}{lll}-2.79726900 & -0.65889200 & -2.26889200\end{array}$ $\begin{array}{lll}-2.28859400 & -1.97788800 & -2.29426000\end{array}$ $\begin{array}{rrr}-2.28859400 & -1.97788800 & -2.29426000 \\ -2.19003700 & 288354900 & -1.79487600\end{array}$ $\begin{array}{lll}-2.00526700 & 0.44257700 & -2.01491300\end{array}$ $\begin{array}{lll}-2.00526700 & 0.44257700 & -2.01491300 \\ -0.98603600 & 0.40799400 & -1.93955300\end{array}$ $\begin{array}{lll}-0.9863500 & 0.40799400 & -1.93955300 \\ -4.12739200 & 1.22231800 & -2.33497300\end{array}$ $\begin{array}{lll}-4.12739200 & 1.22231800 & -2.33497300 \\ -5.40317800 & -0.97973000 & -2.68730900\end{array}$ $\begin{array}{lll}-5.40317800 & -0.97973000 & -2.68730900 \\ -5.27368100 & -1.68900700 & -3.52505700\end{array}$ $\begin{array}{lll}-5.27368100 & -1.68900700 & -3.52505700 \\ -6.21331100 & -0.29499500 & -3.00427200\end{array}$ $\begin{array}{rrr}-6.21331100 & -0.29499500 & -3.00427200 \\ -0.20581000 & 2.28452200 & -0.41119400\end{array}$ $\begin{array}{ccc}-0.20581000 & 2.28452200 & -0.41119400 \\ -0.68852100 & 1.47300700 & 0.05826300\end{array}$ $\begin{array}{rrr}-0.68852100 & 1.47300700 & 0.05826300 \\ 1.13683800 & 0.07146700 & 1.04900900\end{array}$ $\begin{array}{lll}1.13683800 & 0.07146700 & 1.04900900 \\ 1.25656300 & 0.03062500 & 0.01357300\end{array}$ $\begin{array}{rrr}1.25656300 & 0.03062500 & 0.01357300 \\ -0.55944000 & -1.83220900 & -0.51767300\end{array}$ $\begin{array}{lll}-0.55944000 & -1.83220900 & -0.51767300\end{array}$ $\begin{array}{lll}-1.09423400 & -1.14704700 & 0.07120500\end{array}$ $-5.86607800-1.74739000-1.43998100$ $-6.08854700-1.05571400-0.60443500$ $\begin{array}{lll}-5.08837000 & -2.45283600 & -1.09069700\end{array}$ $\begin{array}{lll}-6.78281700 & -2.32937700 & -1.65795200\end{array}$ $\begin{array}{llll}-3.07344300 & -3.05650100 & -2.94551400\end{array}$ $\begin{array}{lll}-3.45468000 & -4.21045100 & -2.21994900\end{array}$ $\begin{array}{lll}-4.17905900 & -5.25088000 & -2.80985200\end{array}$ $\begin{array}{lll}-4.15496400 & -4.03126500 & -4.91546200\end{array}$ $\begin{array}{lll}-3.44115200 & -2.98945200 & -4.30678000\end{array}$ $-3.44115200-2.98945200-4.30678000$ $-3.04777000-4.34524800-0.80478900$ $\begin{array}{lll}-3.04777000 & -4.34524800 & -0.80478900\end{array}$ $-2.02185400-4.53085800-0.74936500$ $\begin{array}{llll}-2.02185400 & -4.53085800 & -0.74936500 \\ -3.53490700 & -5.14631500 & -0.36813500\end{array}$ $-0.24391100-4.25813300-3.34030700$ $-0.24391100-4.25813300-3.34030700$ $\begin{array}{rrr}-1.23242800 & -4.70482000 & -3.56688900 \\ 0.45834100 & -5.11236900 & -3.27726900\end{array}$ $\begin{array}{rrr}-1.45834100 & -5.11236900 & -3.27726900 \\ -1.70506200 & 2.22198400 & 2.35210600\end{array}$ $\begin{array}{rrr}-1.70506200 & 2.22198400 & 2.35210600 \\ 0.17469200 & -3.33088900 & -4.49518200\end{array}$ $\begin{array}{lll}0.17469200 & -3.33088900 & -4.49518200 \\ -0.54465500 & -2.49862000 & -4.63355100\end{array}$ $\begin{array}{rrr}-0.54465500 & -2.49862000 & -4.63355100 \\ 0.22542100 & -3.89461900 & -5.44726900\end{array}$ $\begin{array}{lll}0.22542100 & -3.89461900 & -5.44726900 \\ 1.17023800 & -2.88057300 & -4.30810300\end{array}$ $\begin{array}{lll}1.17023800 & -2.88057300 & -4.30810300\end{array}$ $\begin{array}{llll}1.87328600 & -4.62655400 & -1.02881500\end{array}$ $2.02011700-5.12179500 \quad-0.05193300$ $\begin{array}{lll}1.66480300 & -5.44382100 & -1.74672000\end{array}$ $\begin{array}{lll}1.3 .70328400 & 1.73730000 & 0.93400500\end{array}$ $\begin{array}{lll}3.16769600 & -3.90495700 & -1.44259100\end{array}$ $3.08610200-3.50169800-2.47150200$ $\begin{array}{llll}4.02609200 & -4.60390300 & -1.40965100\end{array}$ $3.37634300-3.04487900-0.77670600$ $\begin{array}{lll}1.76572600 & -3.52828800 & 1.88612600\end{array}$ $\begin{array}{llll}-1.64790800 & 0.29728100 & 0.75689300\end{array}$ $1.00175200-4.68084000 \quad 2.18050000$ $\begin{array}{lll}-0.06858300 & -4.687205400 & 1.92765400\end{array}$ $3.97529400-2.35925200-2.05288000$ $\begin{array}{lll}3.97529400 & -2.35925200 & 2.05288000\end{array}$ $3.83910400-1.73191100 \quad 1.15057000$ $1.836751900-1.61482400 \quad 4.36449800$ $1.70111700-1.08833100-5.25600000$ $-0.09654100-2.02056200 \quad 4.61588700$

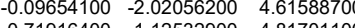
$\begin{array}{lll}-0.71916400 & -1.12532900 & 4.81701100 \\ -0.17453700 & -2.69085700 & 5.49413900\end{array}$ $\begin{array}{lll}-0.17453700 & -2.69085700 & 5.49413900 \\ -0.52391100 & -2.55262000 & 3.74125300\end{array}$ $\begin{array}{rrr}1.81386600 & 1.49986300 & 4.43713600\end{array}$ $\begin{array}{lll}4.40838600 & 2.29584000 & 1.30961800\end{array}$ $\begin{array}{lll}4.36543800 & 1.35869200 & 1.75335200\end{array}$ $\begin{array}{lll}5.40854600 & 2.45202800 & 1.06368200\end{array}$ $\begin{array}{llll}3.93700000 & 2.09539500 & 0.33614500\end{array}$ $\begin{array}{lll}2.12865300 & 5.21788200 & -0.43756000\end{array}$ $\begin{array}{llll}1.74771100 & 6.23548900 & -0.65702600\end{array}$ $3.23628200 \quad 4.85888900-1.44879400$ $\begin{array}{llll}2.85774300 & 4.88564000 & -2.49002700\end{array}$ $\begin{array}{llll}3.63905900 & 3.84162800 & -1.27882800\end{array}$ $\begin{array}{llll}4.06828800 & 5.58682600 & -1.37415000\end{array}$ $\begin{array}{llll}-0.33285000 & 5.50545000 & -2.32023600\end{array}$ $\begin{array}{llll}0.34050400 & 6.33962500 & -2.04261000\end{array}$ $\begin{array}{lll}-1.35860200 & 5.91285700 & -2.23465400\end{array}$ $\begin{array}{llll}-0.05688700 & 5.09434900 & -3.77767100\end{array}$ $\begin{array}{llll}-0.70021000 & 4.25099600 & -4.09860400\end{array}$ $\begin{array}{llll}0.99513200 & 4.77404900 & -3.91484000\end{array}$ $-0.24464900 \quad 5.94513100-4.46194500$ $\begin{array}{llll}-0.24464900 & 5.94513100 & -4.46194500 \\ -3.04540500 & 4.09198500 & -1.99059500\end{array}$ $\begin{array}{lll}-3.04540500 & 4.09198500 & -1.99059500 \\ -3.53177200 & 4.78320200 & -0.85811500\end{array}$ $\begin{array}{llll}-3.53177200 & 4.78320200 & -0.85811500 \\ -4.33415400 & 5.92399400 & -0.98394600\end{array}$ $-4.71265100 \quad 0.44386000-0.08986400$ $-4.71265100 \quad 6.44386600-0.08986400$ $-4.65289000 .40305100-2.26460000$ $-5.27627500 \quad 7.30326300-2.36650800$ $\begin{array}{llll}-4.17962200 & 5.73242100 & -3.40494600\end{array}$ $\begin{array}{llll}-4.43120400 & 6.10541500 & -4.40849200 \\ -3.39102300 & 4.58153000 & -3.26674600\end{array}$ $\begin{array}{lll}-3.39102300 & 4.58153000 & -3.26674600\end{array}$ $\begin{array}{llll}-3.03044000 & 4.04714800 & -4.15746600\end{array}$ $\begin{array}{lll}-3.21527800 & 4.27261100 & 0.48556300\end{array}$ $\begin{array}{lll}-3.70326400 & 4.81953200 & 1.21449100\end{array}$ $\begin{array}{llll}-2.20114600 & 4.32190800 & 0.70568200\end{array}$ $\begin{array}{llll}-3.47585900 & 3.19807500 & 0.64689800\end{array}$ $\begin{array}{lll}-6.16555800 & 2.19305900 & -1.11765500\end{array}$ $\begin{array}{lll}-7.05645300 & 2.83204200 & -1.27518300\end{array}$ $\begin{array}{llll}-5.56728500 & 2.62560500 & -0.29507800\end{array}$ $\begin{array}{llll}-6.52109100 & 1.20182000 & -0.77226700\end{array}$ $\begin{array}{lll}-5.35881400 & 2.08440000 & -2.42302500\end{array}$ $\begin{array}{llll}-6.01140100 & 1.65644500 & -3.21168700\end{array}$ $\begin{array}{llll}-5.10516500 & 3.09461100 & -2.77961400\end{array}$ $\begin{array}{lll}4.07344400 & -0.50727000 & 0.30423100\end{array}$ $\begin{array}{llll}-0.88949000 & 3.14395600 & -1.25676200\end{array}$ $\begin{array}{rrr}-0.88949000 & 3.14395600 & -1.25676200 \\ 3.13631900 & -3.55212200 & 2.23790200\end{array}$ $\begin{array}{lll}3.13631900 & -3.55212200 & 2.23790200 \\ 3.72616100 & -4.68180200 & 2.81763400\end{array}$ $\begin{array}{lll}3.72616100 & -4.68180200 & 2.81763400 \\ 4.79808400 & -4.68179300 & 3.07068300\end{array}$ $\begin{array}{lll}4.79808400 & -4.68179300 & 3.07068300\end{array}$ $\begin{array}{lll}2.94525400 & -5.81723200 & 3.08607400 \\ 3.41128600 & -6.70230300 & 3.54282800\end{array}$ $1.41128600-6.70230300 \quad 3.54282800$

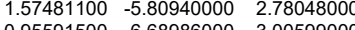
$0.95591500-6.68986000 \quad 3.00599000$ $\begin{array}{lll}3.82048800 & -1.68676200 & 2.83062800\end{array}$ $\begin{array}{lll}2.36946400 & 3.45618600 & 2.09452200\end{array}$ $\begin{array}{llll}3.77885100 & 3.35215300 & 2.09629700\end{array}$

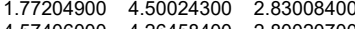
$\begin{array}{llll}4.57406000 & 4.26458400 & 2.80020700\end{array}$ $\begin{array}{llll}2.55964600 & 5.40653700 & 3.55356900\end{array}$ $\begin{array}{llll}0.67626500 & 4.58618800 & 2.83306600\end{array}$ $\begin{array}{lll}3.95998200 & 5.29158500 & 3.53584200\end{array}$ $\begin{array}{llll}5.67114300 & 4.17991300 & 2.77340900\end{array}$ $2.07954400 \quad 6.21132800 \quad 4.12930700$ $\begin{array}{lll}4.58305600 & 6.00644600 & 4.09285700\end{array}$ $\begin{array}{lll}2.50111200 & 2.34992500 & 4.27353200\end{array}$ $\begin{array}{llll}2.28751900 & 0.87802200 & 5.22345000\end{array}$ $\begin{array}{llll}0.45534500 & 2.02202900 & 4.94277000\end{array}$ $\begin{array}{rrr}-0.20011600 & 1.18415800 & 5.25637600\end{array}$ $\begin{array}{lll}0.59756600 & 2.68441000 & 5.81905600\end{array}$ $\begin{array}{lll}-0.08879400 & 2.57751400 & 4.15519500\end{array}$ $\begin{array}{llll}2.55940800 & 5.27678100 & 0.57561700\end{array}$ $3.50872400-0.02269200-1.02707200$ $\begin{array}{lll}3.50872400 & 0.02269200 & -1.02707200 \\ 2.22229000 & -0.65450100 & -1.41656500\end{array}$ $\begin{array}{lll}2.22229000 & -0.65450100 & -1.41656500 \\ 6.21255500 & 1.54077400 & -0.44929300\end{array}$ $\begin{array}{rrr}6.21255500 & 1.54077400 & -0.44929300 \\ 1.97522600 & -253659400 & 4.26280800\end{array}$ $\begin{array}{rrrr}1.97522600 & -2.53659400 & 4.26280800\end{array}$ $-2.50671000-1.151502001 .68417000$ $-4.30447500-0.89655500-2.64538200$ $\begin{array}{lll}-4.30447500 & -0.89655500 & 2.64538200 \\ -6.02285800 & -2.35634400 & 3.10963500\end{array}$ $\begin{array}{lll}-5.23583600 & -3.05798300 & 1.98640800\end{array}$ $\begin{array}{lll}-5.23583600 & -3.05798300 & 1.98640800 \\ -5.83137400 & -3.26100200 & 1.07132200\end{array}$ $\begin{array}{lll}-5.83137400 & -3.26100200 & 1.07132200 \\ -4.79721200 & -4.02632800 & 2.30803400\end{array}$ $\begin{array}{lll}-4.88208200 & -1.65793600 & 3.92395400\end{array}$ $\begin{array}{lll}-6.76213500 & -1.17138900 & 2.45147200\end{array}$ $\begin{array}{lll}-7.48090000 & -0.70129900 & 3.14832900\end{array}$ $\begin{array}{lll}-7.34515500 & -1.49458200 & 1.56657900\end{array}$ $\begin{array}{lll}-6.66358800 & -3.03653500 & 3.70108900\end{array}$ $\begin{array}{lll}-5.39586900 & -0.71869400 & 5.02234100\end{array}$ $\begin{array}{lll}-4.55886600 & -0.22535400 & 5.55729900\end{array}$ $\begin{array}{lll}-6.07759500 & 0.07316300 & 4.66021500\end{array}$ $\begin{array}{lll}-5.95120100 & -1.30733100 & 5.78091000\end{array}$ $\begin{array}{lll}-3.86215200 & -2.62372900 & 4.55113800\end{array}$ $\begin{array}{lll}-3.09417500 & -2.06720000 & 5.12510700\end{array}$ $\begin{array}{lll}-4.36281900 & -3.30580300 & 5.26746900\end{array}$ $\begin{array}{lll}-3.32293500 & -3.25612200 & 3.81648400\end{array}$ $\begin{array}{lll}-3.13935800 & 0.02613600 & 2.96335900\end{array}$ $\begin{array}{lll}-2.23453300 & -0.52585600 & 3.28737200\end{array}$ $\begin{array}{lll}-3.42844100 & 0.72054100 & 3.77776300\end{array}$ $\begin{array}{rrr}-5.428476100 & -0.20356200 & 2.08340400\end{array}$ $\begin{array}{lll}-5.70075100 & 0.78489700 & 2.57266400\end{array}$ $\begin{array}{lll}-5.70075100 & 0.78489700 & 2.57266400 \\ -5.50837200 & 0.01022200 & 1.00351300\end{array}$ $\begin{array}{lll}-3.22015300 & -2.23053500 & 0.85367300\end{array}$ $\begin{array}{lll}-4.53085700 & -5.15890900 & -4.16704000\end{array}$ $\begin{array}{lll}-4.41982600 & -3.96368500 & -5.98073300\end{array}$ $\begin{array}{lll}-4.41982600 & -3.96368500 & -5.98073300 \\ -4.46792600 & -6.13872300 & -2.22572000\end{array}$ $\begin{array}{lll}-4.46792600 & -6.13872300 & -2.22572000 \\ -5.09552600 & -5.97730300 & -4.63679400\end{array}$ $\begin{array}{rrr}-5.09552600 & -5.97730300 & -4.63679400 \\ 6.77788700 & 0.60256800 & -0.99704300\end{array}$ $\begin{array}{rrr}6.77788700 & 0.60256800 & -0.99704300 \\ 6.20426500 & -0.30830600 & -2.08806700\end{array}$ $\begin{array}{rrr}8.20895000 & 0.13695100 & -0.75769500\end{array}$ 
SUPPORTING INFORIMATION

$\begin{array}{lrrr}\text { C } & 6.94321900 & -1.66992800 & -1.76557200 \\ \text { C } & 6.99001900 & 0.17947100 & -3.37140500 \\ \text { C } & 8.36625600 & -1.01079600 & -1.77553700 \\ \text { H } & 8.91877100 & 0.97877200 & -0.89532300 \\ \text { H } & 8.31734500 & -0.18594100 & 0.29890300 \\ \text { C } & 6.74792700 & -2.74601800 & -2.84130600 \\ \text { C } & 6.56964800 & -2.29032300 & -0.40708300 \\ \text { H } & 6.50460200 & -0.24961100 & -4.27094500 \\ \text { H } & 6.92792800 & 1.28151600 & -3.47394300 \\ \text { C } & 8.43563400 & -0.35406600 & -3.17250900 \\ \text { H } & 9.19647100 & -1.70647000 & -1.54916400 \\ \text { H } & 8.71134600 & -1.08522200 & -3.95590300 \\ \text { H } & 9.19312200 & 0.45284500 & -3.20676400 \\ \text { C } & 4.70152400 & -0.32524800 & -2.36035800 \\ \text { H } & 4.38858200 & -1.31626300 & -2.74478800 \\ \text { H } & 4.40640700 & 0.43138900 & -3.11487000 \\ \text { O } & 3.32448300 & 1.52630500 & -0.93741700 \\ \text { H } & 6.94651600 & -2.39658300 & -3.87131800 \\ \text { H } & 5.71393900 & -3.14935300 & -2.82218700 \\ \text { H } & 7.42654100 & -3.60220700 & -2.64915900 \\ \text { H } & 5.55246200 & -2.72766700 & -0.45699200 \\ \text { H } & 6.58977100 & -1.56123100 & 0.42678000 \\ \text { H } & 7.27304200 & -3.11257000 & -0.16310400\end{array}$

200

\section{Conformer D}

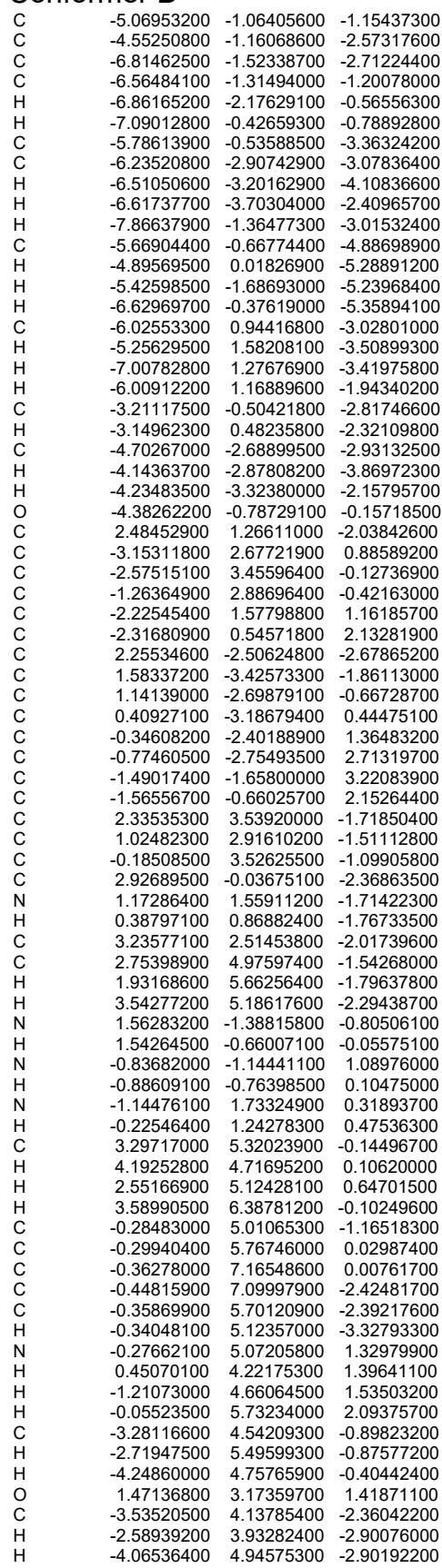

$\begin{array}{rrr}-4.16013400 & 3.22372500 & -2.41824700\end{array}$ $\begin{array}{lll}-4.52638000 & 2.94083300 & 1.45007000\end{array}$ $\begin{array}{lll}-4.56798800 & 2.69238500 & 2.52340300 \\ -4.70936200 & 4.03310600 & 1.39979000\end{array}$ $\begin{array}{rrr}-4.70936200 & 4.03310600 & 1.39979000\end{array}$ $\begin{array}{rrr}0.72028300 & 1.63347900 & 3.26669100 \\ -5.66519700 & 2.22236000 & 0.71077100\end{array}$ $\begin{array}{rrr}-5.66519700 & 2.22236000 & 0.71077100 \\ -5.70494600 & 2.52276300 & -0.35535500\end{array}$ $\begin{array}{llr}-5.70494600 & 2.52276300 & -0.35535500 \\ -6.64183600 & 2.47433700 & 1.16844000\end{array}$ $\begin{array}{lll}-6.64183600 & 2.47433700 & 1.16844000 \\ -5.53952600 & 1.12318800 & 0.74008800\end{array}$ $\begin{array}{lll}-5.53952600 & 1.12318800 & 0.74008800 \\ -3.28538200 & 0.78338500 & 3.24050500\end{array}$ $\begin{array}{rrr}-3.28538200 & 0.78338500 & 3.24050500 \\ 1.28479600 & 0.71055000 & 0.99428800\end{array}$ $\begin{array}{rrr}1.28479600 & 0.71055000 & 0.99428800 \\ -3.02008800 & 1.78021000 & 4.20144900\end{array}$ $\begin{array}{lll}-3.07260900 & 2.33445500 & 4.12893400\end{array}$ $\begin{array}{lll}-4.72823600 & -1.06981300 & 2.43295400\end{array}$ $\begin{array}{lll}-4.67957200 & -0.84280700 & 1.34904200\end{array}$ $\begin{array}{lll}-4.00430700 & -1.80168300 & 2.58300200\end{array}$ $\begin{array}{lll}-4.00430700 & -1.80168300 & 2.58300200 \\ -1.83468900 & -1.47608500 & 4.67720200\end{array}$ $\begin{array}{lll}-1.88329900 & -2.47110900 & 5.16231500\end{array}$ $\begin{array}{lll}-1.88329900 & -2.47110900 & 5.16231500 \\ -0.78829900 & -0.60358200 & 5.39901400\end{array}$ $\begin{array}{rrr}-0.18829900 & -0.60358200 & 5.39901400 \\ 0.19837400 & -1.10815000 & 5.41727900\end{array}$ $\begin{array}{rrr}-1.09522000 & -0.42069000 & 6.44750800\end{array}$ $\begin{array}{rrr}-1.09522000 & -0.42069000 & 6.44750800 \\ -0.64457000 & 0.37031600 & 4.89281500\end{array}$ $\begin{array}{lll}-0.36597400 & -3.96085200 & 3.51702900\end{array}$ $\begin{array}{lll}-0.36597400 & -3.96085200 & 3.51702900 \\ -2.02486000 & -4.67807800 & 0.02205700\end{array}$ $\begin{array}{rrr}-2.02486000 & -4.67807800 & 0.02205700 \\ -1.90684700 & -3.74726400 & -0.58228000\end{array}$ $\begin{array}{lll}-2.53414900 & -4.37231500 & 0.8708950\end{array}$ $\begin{array}{rrr}-2.66476700 & -5.29871600 & -0.50292500 \\ 1.25173100 & -4.83144400 & -2.28638400\end{array}$ $\begin{array}{lll}1.25173100 & -4.83144400 & -2.28638400 \\ 2.03526600 & -5.16969300 & -2.99386800\end{array}$ $\begin{array}{rrr}2.03526600 & -5.16969300 & -2.99386800 \\ -0.11921400 & -4.96432700 & -2.97307600\end{array}$ $\begin{array}{rrr}-0.11921400 & -4.96432700 & -2.97307600 \\ -0.14953600 & -4.41521100 & -3.93269100\end{array}$ $\begin{array}{lll}-0.14953600 & -4.41521100 & -3.93269100 \\ -0.92957200 & -4.52776400 & -2.35971300\end{array}$ $\begin{array}{lll}-0.92957200 & -4.52776400 & -2.35971300\end{array}$ $\begin{array}{lll}-0.34640200 & -6.03069700 & -3.16941300\end{array}$ $\begin{array}{lll}2.65645700 & -2.73340400 & -4.11477600\end{array}$ $\begin{array}{lll}2.56127400 & -3.81384900 & -4.33875500\end{array}$ $\begin{array}{lll}3.72504400 & -2.49032600 & -4.28429400\end{array}$ $\begin{array}{lll}1.78754800 & -1.93150000 & -5.10108700 \\ 1.90962200 & -0.83920200 & -4.95679900\end{array}$ $\begin{array}{lll}0.71379900 & -2.15926100 & -4.96267800\end{array}$ $2.07637500-2.16755000-6.14416300$ $\begin{array}{lll}2.16301800 & -0.20191400 & -3.17135300\end{array}$ $\begin{array}{lll}5.21196100 & -1.05830200 & -2.75639000\end{array}$ $\begin{array}{lll}5.37916700 & -1.21787400 & -3.51003400\end{array}$ $\begin{array}{lll}7.18520300 & -1.88293000 & -3.16195100\end{array}$ $\begin{array}{lll}7.18520300 & -1.88293000 & -3.16195100 \\ 6.51481400 & -0.52939700 & -4.72710500\end{array}$ $\begin{array}{lll}7.42929500 & -0.65856600 & -5.32404500\end{array}$ $\begin{array}{lll}7.42929500 & -0.65856600 & -5.32404500 \\ 5.48099700 & 0.30782500 & -5.17711200\end{array}$ $\begin{array}{llll}5.57641400 & 0.83634000 & -6.13684300\end{array}$ $\begin{array}{lll}4.572582400 & 0.47359200 & -4.40206600\end{array}$ $\begin{array}{lll}4.32582400 & 0.47359200 & -4.40206600 \\ 3.51504700 & 1.12914900 & -4.75288400\end{array}$ $\begin{array}{rrr}3.51504700 & 1.12914900 & -4.75288400 \\ 5.06459800 & -1.84934000 & -1.51828700\end{array}$ $\begin{array}{lll}5.06459800 & -1.84934000 & -1.51828700 \\ 5.95898700 & -2.30873200 & -1.27724600\end{array}$ $\begin{array}{lll}5.95898700 & -2.30873200 & -1.27724600 \\ 4.34832700 & -2.59667100 & -1.65871100\end{array}$ $\begin{array}{lll}4.34832700 & -2.59667100 & -1.65871100 \\ 4.71652100 & -1.28122900 & -0.64232200\end{array}$ $\begin{array}{rrr}4.71652100 & -1.28122900 & -0.64232200 \\ 5.49822400 & 2.15873300 & -0.89753200\end{array}$ $\begin{array}{lll}5.49822400 & 2.15873300 & -0.89753200 \\ 6.58726800 & 2.31256800 & -1.02920400\end{array}$ $\begin{array}{lll}6.58726800 & 2.31256800 & -1.02920400 \\ 5.31439600 & 1.07879400 & -0.74296800\end{array}$ $\begin{array}{rrr}5.31439600 & 1.07879400 & -0.74296800 \\ 5.19673700 & 2.68091300 & 0.03354100\end{array}$ $\begin{array}{lll}4.72805500 & 2.69600600 & -2.11472800\end{array}$ $\begin{array}{llll}4.94089400 & 3.77683100 & -2.22660500\end{array}$ $\begin{array}{llll}5.12649800 & 2.22354800 & -3.03066900\end{array}$ $\begin{array}{lll}-1.04408500 & -2.00607800 & -3.39106900\end{array}$ $\begin{array}{lll}2.24942200 & -1.22989000 & -1.98547700\end{array}$ $\begin{array}{lll}-4.48615800 & 0.05408400 & 3.35640000\end{array}$ $\begin{array}{lll}-5.42016100 & 0.32462300 & 4.36239900\end{array}$ $\begin{array}{lll}-6.35610900 & -0.25154700 & 4.43296100\end{array}$ $\begin{array}{lll}-5.14392900 & 1.33492100 & 5.29937500\end{array}$ $\begin{array}{lll}-5.87383300 & 1.55074700 & 6.09304300\end{array}$ $\begin{array}{lll}-5.87383300 & 1.55074700 & 6.09304300 \\ -3.93400 & 2.04915500 & 5.22887600\end{array}$ $\begin{array}{lll}-3.93643400 & 2.04915500 & 5.22887600 \\ -3.71128400 & 2.82473800 & 5.97554400\end{array}$

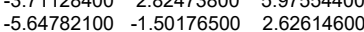
$\begin{array}{llll}0.38495800 & -4.66530200 & 0.62984000\end{array}$ $\begin{array}{rrr}0.38495800 & -4.66530200 & 0.62984000 \\ -0.79476700 & -5.39950700 & 0.39166800\end{array}$ $\begin{array}{ccc}1.54314100 & -5.38097100 & 0.99780600\end{array}$ $\begin{array}{rrr}1.54314100 & -5.38097100 & 0.99780600 \\ -0.83287700 & -6.79407100 & 0.50626200\end{array}$ $\begin{array}{rrr}-0.83287700 & -6.79407100 & 0.50626200 \\ 1.51687800 & -6.77522800 & 1.13845100\end{array}$ $\begin{array}{lll}1.51687800 & -6.77522800 & 1.13845100 \\ 2.47569400 & -4.82423600 & 1.16877800\end{array}$ $\begin{array}{lll}2.47569400 & -4.82423600 & 1.16877800 \\ 0.32998900 & -7.48372400 & 0.88583400\end{array}$ $\begin{array}{rrr}0.32998900 & -7.48372400 & 0.88583400 \\ -1.76097100 & -7.35022200 & 0.30157800\end{array}$ $\begin{array}{rrr}-1.76097100 & -7.35022200 & 0.30157800 \\ 2.42815700 & -7.31571900 & 1.43340400\end{array}$ $\begin{array}{lll}0.30473300 & -8.57908400 & 0.97962100\end{array}$ $\begin{array}{lll}-0.47312100 & -4.88859000 & 2.92401800\end{array}$ $\begin{array}{lll}-1.06386600 & -4.07238800 & 4.37026600\end{array}$ $\begin{array}{lll}1.07065900 & -3.86706100 & 4.05597600\end{array}$ $\begin{array}{lll}1.17989600 & -3.01131400 & 4.75044300\end{array}$ $\begin{array}{lll}1.34377100 & -4.78888000 & 4.60604400\end{array}$ $\begin{array}{lll}1.80061000 & -3.73200900 & 3.23299600\end{array}$ $1.31544900-5.52828600-1.43409000$ $\begin{array}{llll}-1.72795700 & -1.41124000 & -2.22188800\end{array}$ $-2.21618000-2.43010900-1.18484500$ $-0.91036300-0.33424600-1.49509700$ $\begin{array}{lll}-2.83506200 & -1.02823000 & 4.81887300\end{array}$ $\begin{array}{rrr}-1.56336000 & 1.78287200 & 2.05077500\end{array}$ $\begin{array}{rrr}1.543443300 & -0.60390000 & 1.76448600\end{array}$ $\begin{array}{lrr}4.85139700 & 0.30030800 & 2.87340600\end{array}$ $\begin{array}{llll}5.24562000 & -1.21986900 & 3.88491000\end{array}$ $\begin{array}{lll}5.24562000 & -1.21986900 & 3.88491000 \\ 5.25532100 & -1.64412500 & 2.40059200\end{array}$ $\begin{array}{lll}4.255349500 & -2.67235500 & 2.21819100\end{array}$ $\begin{array}{lll}4.87549500 & -2.67235500 & 2.21819100 \\ 6.26244500 & -1.58968200 & 1.93641000\end{array}$ $\begin{array}{rrr}5.15645600 & 0.34053800 & 3.78471800\end{array}$ $\begin{array}{rrr}5.15645600 & 0.34053800 & 3.78471800 \\ 3.86017200 & -1.61032300 & 4.44356900\end{array}$ $\begin{array}{lll}3.86017200 & -1.61032300 & 4.44356900 \\ 3.81466400 & -1.48374700 & 5.54141900\end{array}$ $\begin{array}{lll}3.62794500 & -2.67282700 & 4.23844300\end{array}$ $\begin{array}{lll}6.10541800 & -1.60204900 & 4.46593700\end{array}$ $\begin{array}{llll}4.93788500 & 1.03770900 & 5.13257500\end{array}$ $\begin{array}{llll}4.85130400 & 2.13648200 & 5.00776500\end{array}$ $\begin{array}{llll}4.04205400 & 0.69311600 & 5.68182500\end{array}$ $\begin{array}{lll}5.81575700 & 0.86220200 & 5.78752400\end{array}$ $\begin{array}{lll}6.34671400 & 1.01078600 & 3.07688800\end{array}$ $\begin{array}{lll}6.19538700 & 2.10730300 & 3.00716900\end{array}$ $\begin{array}{lll}7.28089600 & 0.85175300 & 3.65235800\end{array}$ $\begin{array}{lll}6.52994300 & 0.64704300 & 2.04506300\end{array}$ $\begin{array}{llll}3.32683100 & 1.65988600 & 2.45027500\end{array}$ $\begin{array}{lll}3.83083500 & 2.03323300 & 1.53800600\end{array}$ $\begin{array}{lll}3.45279000 & 2.40785200 & 3.25834200\end{array}$ $\begin{array}{lll}2.89771900 & -0.62950500 & 3.71418000\end{array}$ $\begin{array}{llll}2.31661700 & 0.00809800 & 4.40754300\end{array}$ $\begin{array}{lll}2.15166900 & -1.12813100 & 3.406327300\end{array}$ $\begin{array}{lll}4.09008200 & -0.49253400 & 0.06327300\end{array}$ $\begin{array}{rrr}4.09008200 & -0.49253400 & 0.55689100 \\ -0.43909300 & 7.83294400 & -1.22612800\end{array}$ $\begin{array}{ccc}-0.43909300 & 7.83294400 & -1.22612800 \\ -0.51379900 & 7.62244700 & -3.39036800\end{array}$ $\begin{array}{lll}-0.51379900 & 7.62244700 & -3.39036800 \\ -0.35939700 & 7.74148500 & 0.94622800\end{array}$ $\begin{array}{llll}-0.35939700 & 7.74148500 & 0.94622800 \\ -0.49409900 & 8.93114400 & -1.24483600\end{array}$

$\begin{array}{rrr}-0.49409900 & 8.93114400 & -1.24483600 \\ -3.01346300 & -0.37997100 & -3.89987100\end{array}$

200

\section{Conformer E}

\begin{tabular}{|c|c|c|c|}
\hline C & 3.10275700 & 1.45297900 & -3.08939600 \\
\hline C & 3.65925200 & 0.11472500 & -3.62537900 \\
\hline & 5.01021900 & 1.73059400 & -4.52831100 \\
\hline & 4.02141700 & 2.53214500 & -3.66136000 \\
\hline & 3.42928900 & 3.28999400 & -4.21411900 \\
\hline & 4.51273900 & 3.06815000 & -2.82471500 \\
\hline & 5.20981000 & 0.42353800 & -3.68826200 \\
\hline & 4.22359700 & 1.24031500 & -5.76316200 \\
\hline & 4.89013300 & 0.78891700 & -6.52213700 \\
\hline & 3.68423100 & 2.06893900 & -6.26178200 \\
\hline & 5.94696500 & 2.27133000 & -4.76198400 \\
\hline & 6.02306700 & -0.65778100 & -4.41240000 \\
\hline & 6.11166600 & -1.57714700 & -3.79619600 \\
\hline & 5.61365400 & -0.95478800 & -5.39624600 \\
\hline & 7.05782800 & -0.29690800 & -4.58415900 \\
\hline & 5.86113400 & 0.67361300 & -2.31876500 \\
\hline & 5.98824000 & -0.27741000 & -1.76577700 \\
\hline & 6.86919600 & 1.11673200 & -2.45209100 \\
\hline & 5.27262300 & 1.34164500 & -1.66224000 \\
\hline & 3.21016000 & -1.21925500 & -3.02976400 \\
\hline & 4.00127200 & -1.98748400 & -3.14572200 \\
\hline & 3.25865600 & 0.18647500 & -5.15301300 \\
\hline & 3.39148500 & -0.81548400 & -5.60920500 \\
\hline & 2.18819500 & 0.44926000 & -5.26632100 \\
\hline & 2.10808400 & 1.64732900 & -2.40419000 \\
\hline & -0.56994600 & 3.01514300 & -0.49052300 \\
\hline & -1.17697900 & -2.98203900 & -2.56631200 \\
\hline & -1.56649500 & -1.84327000 & -3.28616300 \\
\hline & -1.67888600 & -0.75113800 & -2.33461600 \\
\hline & -1.05419200 & -2.58186100 & -1.16612200 \\
\hline & -0.67327000 & -3.36458600 & -0.04797800 \\
\hline & 2.82412900 & 2.90116600 & 1.36983700 \\
\hline & 3.37633600 & 1.77176300 & 1.98555000 \\
\hline & 2.38855400 & 0.69310500 & 1.87504700 \\
\hline & 2.38631100 & -0.57739900 & 2.49671300 \\
\hline & 1.41620400 & -1.60185900 & 2.29208800 \\
\hline & 1.09575100 & -2.76658000 & 3.11694700 \\
\hline & 0.15114200 & -3.52806600 & 2.40565100 \\
\hline & -0.07127500 & -2.85521100 & 1.13589600 \\
\hline & -2.57294200 & 2.93564500 & -1.62709300 \\
\hline & -1.82044600 & 1.70802000 & -1.85627300 \\
\hline & -2.19970700 & 0.55526400 & -2.58036400 \\
\hline & 0.50605900 & 3.39108700 & 0.34109300 \\
\hline & -0.62474300 & 1.81452800 & -1.17292700 \\
\hline & 0.20432300 & 1.21964800 & -1.35453900 \\
\hline & -1.81176900 & 3.73993700 & -0.77711300 \\
\hline & -3.96672100 & 3.26881600 & -2.08876600 \\
\hline & -4.05868700 & 3.12725000 & -3.18115700 \\
\hline & -4.13699900 & 4.34958800 & -1.91822000 \\
\hline & 1.33335600 & 1.18490100 & 1.12535500 \\
\hline & 0.38036500 & 0.74647000 & 1.06380500 \\
\hline & 0.63843700 & -1.67837800 & 1.16243100 \\
\hline $\mathrm{H}$ & 0.85467800 & -1.16579800 & 0.26395700 \\
\hline & -1.34723800 & -1.23444800 & -1.09283200 \\
\hline $\mathrm{H}$ & -1.48893100 & -0.69280100 & -0.20464000 \\
\hline & -5.077744300 & 2.47931200 & -1.38152800 \\
\hline $\mathrm{H}$ & -5.06440700 & 2.66021000 & -0.28943700 \\
\hline & -4.96097500 & 1.39081000 & -1.53909100 \\
\hline $\mathrm{H}$ & -6.07256700 & 2.77638000 & -1.76734000 \\
\hline & -3.25155400 & 0.64375600 & -3.62111800 \\
\hline C & -4.39539400 & -0.19094700 & -3.57220900 \\
\hline & -5.39255200 & -0.13577500 & -4.55096200 \\
\hline C & -4.12738500 & 1.59376100 & -5.70408500 \\
\hline & -3.14230400 & 1.53797000 & -4.70865800 \\
\hline $\mathrm{H}$ & -2.25473900 & 2.18497400 & -4.77005000 \\
\hline & -4.53926700 & -1.17265700 & -2.47529700 \\
\hline $\mathrm{H}$ & -4.34754400 & -0.79644100 & -1.457111600 \\
\hline 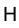 & -3.85882400 & -1.95328400 & -2.60514500 \\
\hline $\mathrm{H}$ & -5.48880700 & -1.58274300 & -2.48706900 \\
\hline C & -1.59479700 & -1.70970900 & -4.78678500 \\
\hline $\mathrm{H}$ & -2.54875500 & -1.26676200 & -5.13437600 \\
\hline H & -1.55416500 & -2.71949200 & -5.24021700 \\
\hline $\mathrm{O}$ & -0.53017600 & 0.44862700 & 3.47778100 \\
\hline C & -0.42367200 & -0.86195200 & -5.31265700 \\
\hline $\mathrm{H}$ & -0.42755600 & 0.15660300 & -4.87386200 \\
\hline $\mathrm{H}$ & -0.47294500 & -0.76286500 & -6.41469200 \\
\hline $\mathrm{H}$ & 0.54826400 & -1.33125700 & -5.05948200 \\
\hline & -0.72291700 & -4.26751300 & -3.20258600 \\
\hline
\end{tabular}


SUPPORTING INFORIMATION

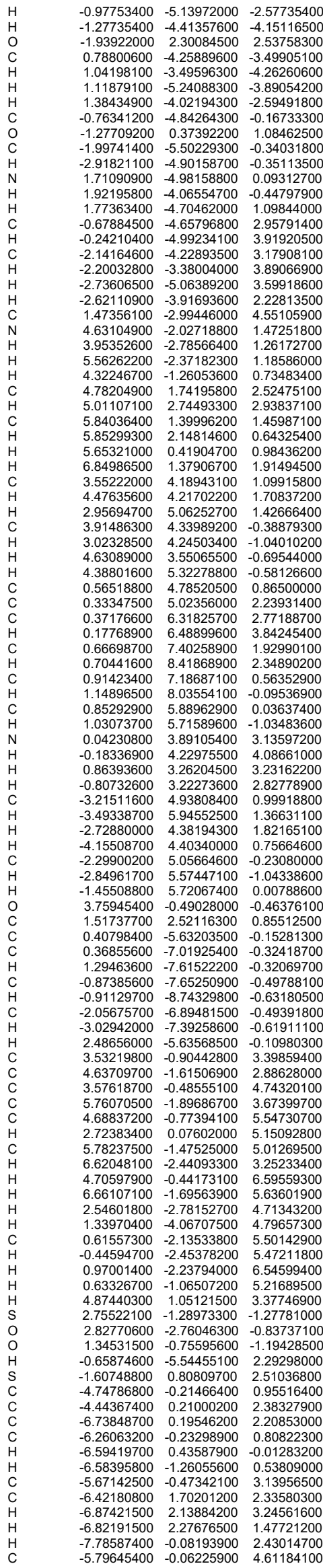

$\begin{array}{lll}-5.01044500 & -0.53766100 & 5.23346900\end{array}$ $\begin{array}{lll}-5.74676600 & 1.02902800 & 4.78395100\end{array}$ $\begin{array}{lll}-6.76959900 & -0.41360900 & 5.0116480\end{array}$ $\begin{array}{lll}-5.62770000 & -2.00959700 & 3.0672400\end{array}$ $\begin{array}{lll}-4.84676800 & -2.41408500 & 3.74317700\end{array}$ $\begin{array}{lll}-6.59261900 & -2.44266600 & 3.39963700\end{array}$ $\begin{array}{lll}-5.41855100 & -2.41221200 & 2.05495600\end{array}$ $\begin{array}{lll}-3.09490300 & -0.11390800 & 3.00138500\end{array}$ $\begin{array}{lll}-2.79590100 & -1.16956300 & 2.8410000\end{array}$ $\begin{array}{lll}-3.13479600 & 0.06428200 & 4.09500300\end{array}$ $\begin{array}{lll}-4.86990000 & 1.72566000 & 2.40131900\end{array}$ $\begin{array}{lll}-4.48516600 & 2.17709900 & 3.33572200\end{array}$ $\begin{array}{lll}-4.38341200 & 2.28598700 & 1.58203000\end{array}$

$\begin{array}{lll}-3.93645200 & -0.49476800 & 0.05887500\end{array}$

$\begin{array}{lll}-5.25628800 & 0.76182000 & -5.62349300\end{array}$

$\begin{array}{lll}-4.01461200 & 2.28990900 & -6.54800200\end{array}$

$\begin{array}{lll}-6.27674400 & -0.78978000 & -4.49136300\end{array}$

$\begin{array}{llll}-6.03615800 & 0.80287700 & -6.39770800\end{array}$

\section{Conformer $\mathbf{F}$}

$\begin{array}{lll}-5.03020800 & -2.08569500 & -3.78559700\end{array}$ $\begin{array}{lll}-4.85881500 & -0.91341000 & -2.78052400\end{array}$ $-7.10866200-1.16660400-3.06162300$ $\begin{array}{lll}-6.53119700 & -2.23279400 & -4.01464900 \\ -6.78276700 & -2.07029800 & -5.08323100\end{array}$ $\begin{array}{lll}-6.78276700 & -2.07029800 & -5.08323100 \\ -6.83823800 & -3.27240200 & -3.77811800\end{array}$ $\begin{array}{lll}-6.83823800 & -3.27240200 & -3.77811800 \\ -6.10185700 & -1.18555200 & -1.85132600\end{array}$ $\begin{array}{lll}-6.10185700 & -1.18555200 & -1.85132600 \\ -6.83589000 & 0.21493900 & -3.70373400\end{array}$ $\begin{array}{llll}-6.83589000 & 0.21493900 & -3.70373400\end{array}$ $\begin{array}{llll}-7.36900900 & 1.02574000 & -3.17114300\end{array}$ $\begin{array}{llll}-7.17920900 & 0.24848400 & -4.75580300\end{array}$ $\begin{array}{lll}-8.16301600 & -1.34009700 & -2.77335800\end{array}$ $\begin{array}{lll}-6.41525800 & -0.08784800 & -0.82089800\end{array}$ $\begin{array}{lll}-5.75994000 & -0.21256300 & 0.06362900\end{array}$ $\begin{array}{llll}-6.28398100 & 0.93740000 & -1.21323400\end{array}$ $\begin{array}{lll}-7.46566900 & -0.18928600 & -0.47850800\end{array}$ $\begin{array}{lll}-6.03319000 & -2.53026800 & -1.11068200\end{array}$ $\begin{array}{llll}-5.26191700 & -2.47840300 & -0.31420400\end{array}$ $\begin{array}{lll}-7.00369300 & -2.75930800 & -0.62543400\end{array}$

$\begin{array}{lll}-5.77585200 & -3.39120500 & -1.75795500\end{array}$

$\begin{array}{lll}-3.41784700 & -0.93196200 & -2.31294200\end{array}$

$\begin{array}{lll}-2.45004700 & -0.56055700 & -3.11602700 \\ -5.29095100 & 0.36114800 & -3.57588400\end{array}$ $-4.99181400 \quad 1.26516000-3.00919200$ $\begin{array}{lll}-4.99181400 & 1.26516600 & -3.00919200\end{array}$ $-4.77335200-0.41117100-4.55540000$ $-4.12605400-2.76533500-4.23804500$ $2.40928600-0.20055900-2.36151300$ $\begin{array}{lll}-1.58541800 & 4.12039300 & 0.22766700\end{array}$ $\begin{array}{ccc}-0.70181000 & 4.37542900 & -0.83047700 \\ 0.25658800 & 3.28400000 & -0.86859200\end{array}$ $\begin{array}{rrr}-0.25658800 & 3.28400000 & -0.86859200 \\ -1.16434900 & 285796700 & 0.84450300\end{array}$ $\begin{array}{rrr}-1.16434900 & 2.85796700 & 0.84450300 \\ -1.64983400 & 2.22413200 & 2.01549600 \\ 0.29579800 & -3.37551000 & -1.89113500\end{array}$

$\begin{array}{rrr}-1.64983400 & 2.22413200 & 2.01549600 \\ 0.29579800 & 3.37551000 & -1.89113500\end{array}$

$\begin{array}{rrr}0.29579800 & -3.37551000 & -1.89113500 \\ -0.41888700 & -3.69752600 & -0.72713200\end{array}$ $\begin{array}{rrr}-0.41888700 & -3.69752600 & -0.72713200 \\ -0.13334800 & -2.65359300 & 0.25751000\end{array}$ $\begin{array}{lll}-0.13334800 & -2.65359300 & 0.25751000 \\ -0.65261000 & -250957200 & 1.57334500\end{array}$

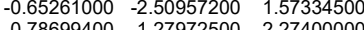
$\begin{array}{lll}-0.78699400 & -1.27972500 & 2.27400000\end{array}$ $\begin{array}{lll}-1.17036400 & -1.05157200 & 3.66276100\end{array}$ $\begin{array}{lll}-1.49473300 & 0.31014300 & 3.78150100\end{array}$ $\begin{array}{lll}-1.29638500 & 0.91854200 & 2.46671600\end{array}$ $\begin{array}{llll}3.50630400 & 1.82040400 & -2.42425300\end{array}$ $\begin{array}{lll}2.12717700 & 2.01883300 & -1.98336300\end{array}$ $\begin{array}{llll}1.45391100 & 3.21223000 & -1.64148500\end{array}$ $\begin{array}{lll}2.06343200 & -1.57159900 & -2.41838700\end{array}$ $\begin{array}{lll}1.52444800 & 0.77603500 & -1.94936400\end{array}$ $\begin{array}{llll}0.52245400 & 0.60947800 & -1.76753500\end{array}$ $\begin{array}{llll}3.67119200 & 0.45389700 & -2.67575200\end{array}$ $\begin{array}{llll}4.63233000 & 2.81928900 & -2.48036300\end{array}$ $4.632330002 .81928900-2.48036300$ $\begin{array}{llll}5.21463400 & 2.62982800 & -3.40685000\end{array}$ $\begin{array}{lll}5.21463400 & 2.62982800 & -3.40685000 \\ 0.73433700 & -1.75428800 & -0.33429700\end{array}$ $\begin{array}{rrrr}0.73433700 & -1.75428800 & -0.33429700 \\ 1.24893700 & -0.99051100 & 0.16929800\end{array}$ $\begin{array}{rrr}1.24893700 & -0.99051100 & 0.16929800 \\ -0.79866400 & -0.05921200 & 1.63797500\end{array}$ $\begin{array}{rrr}-0.79866400 & -0.05921200 & 1.63797500 \\ -0.86483700 & 0.00640700 & 0.59237700\end{array}$ \begin{tabular}{rrr}
-0.86483700 & 0.00640700 & 0.59237700 \\
-0.08815700 & 2.38466500 & 0.11372800 \\
\hline
\end{tabular} $\begin{array}{ccc}-0.08815700 & 2.38466500 & 0.11372800 \\ 0.56233200 & 1.60942100 & 0.41730900\end{array}$

$\begin{array}{lll}0.56233200 & 1.60942100 & 0.41730900 \\ 5.57288500 & 2.76238600 & -1.26181200\end{array}$ $\begin{array}{lll}5.57288500 & 2.76238600 & -1.26181200 \\ 6.07283500 & 1.77733100 & -1.17203900\end{array}$ $\begin{array}{llll}6.07283500 & 1.77733100 & -1.17203900 \\ 5 & .01607300 & 2.95115400 & -0.32424300\end{array}$ $\begin{array}{lll}5.01607300 & 2.95115400 & -0.32424300\end{array}$ $\begin{array}{llll}6.36994500 & 3.52543500 & -1.36180400\end{array}$ $\begin{array}{llll}2.05213200 & 4.52338400 & -2.00296700\end{array}$ $\begin{array}{llll}2.44096100 & 5.42941000 & -0.98711200\end{array}$ $3.01026100 \quad 6.67023500-1.29465100$ $2.79263500 \quad 6.15865900-3.66515300$ $2.24359600 \quad 4.90935800 \quad-3.34690400$ $\begin{array}{lll}1.95126600 & 4.21188900 & -4.14532700\end{array}$ $\begin{array}{llll}2.24416800 & 5.06391800 & 0.42775900\end{array}$ $\begin{array}{llll}2.54746700 & 4.01607400 & 0.67007000\end{array}$ $\begin{array}{lll}1.24436200 & 5.15543700 & 0.69681800\end{array}$ $\begin{array}{llll}2.78545700 & 5.69278000 & 1.04509100\end{array}$ $\begin{array}{rrr}-0.87270800 & 5.45290400 & -1.86872400\end{array}$ $\begin{array}{llll}0.05200400 & 6.04966800 & -1.99202200\end{array}$ $-1.04174800 \quad 6.16770200-1.51708800$ $3.061300 \quad 2.65901800 \quad 0.80690500$ $\begin{array}{lll}3.06621300 & 2.65901800 & 0.86690500\end{array}$ $-0.51589200-4.20719400-3.35064400$ $-1.46800700 \quad 5.69011500-3.05214800$ $\begin{array}{lll}-1.46800700 & 5.69011500 & -3.96214800\end{array}$ $\begin{array}{lll}-2.22826300 & 4.28321700 & -3.14745400\end{array}$ $\begin{array}{rrr}-2.77325300 & 5.00061400 & 0.52185300 \\ -3.04371000 & 4.97116700 & 1.58917800\end{array}$ $\begin{array}{lll}-2.46652900 & 6.04952400 & 0.33135900\end{array}$ $\begin{array}{lll}2.15207600 & 1.65712900 & 3.00016400\end{array}$ $\begin{array}{lll}-4.01255900 & 4.69868600 & -0.34057000\end{array}$ $\begin{array}{lll}-3.79584400 & 4.79673800 & -1.42238700 \\ -4.82883400 & 5.40482700 & -0.09210000\end{array}$ $\begin{array}{lll}-4.82883400 & 5.40482700 & -0.09210000 \\ -4.39340800 & 3.67106100 & -0.19096400\end{array}$ $\begin{array}{lll}-2.68941800 & 2.95623400 & 2.80191400\end{array}$ $\begin{array}{lll}1.77969300 & 0.51894000 & 0.78904400\end{array}$ $\begin{array}{lll}-2.36070700 & 3.96904400 & 3.72413700\end{array}$ $\begin{array}{lll}-1.30147600 & 4.20519500 & 3.89835000\end{array}$ $\begin{array}{lll}-4.42302900 & 1.66329900 & 1.58030200\end{array}$ $\begin{array}{llll}-5.44980100 & 1.56043100 & 1.50739600\end{array}$ $\begin{array}{llll}-4.05464100 & 1.82555600 & 0.56846500\end{array}$ $\begin{array}{lll}-1.73594000 & 1.03072900 & 5.07522600\end{array}$ $\begin{array}{llll}-2.08545700 & 0.30773700 & 5.83855600\end{array}$ $\begin{array}{lll}-0.45096900 & 1.72373200 & 5.537165500\end{array}$ $0.33431300 \quad 0.97977600 \quad 5.81200100$ $\begin{array}{ccc}0.33431300 & 0.97977600 & 5.81200100 \\ -0.65600700 & 2.31298400 & 6.48708100\end{array}$ $\begin{array}{llll}-0.65192500 & 2.39727300 & 4.48708100 \\ -0.02192500 & 2.357100\end{array}$ $-0.02192500 \quad 2.39727300 \quad 4.80357100$ $\begin{array}{lll}-1.05614600 & -2.02433000 & 4.80927300\end{array}$ $\begin{array}{lll}-3.47801900 & -2.63593000 & 2.19023700\end{array}$ $\begin{array}{lll}-3.25055100 & -2.05772700 & 1.30408900\end{array}$ $\begin{array}{lll}-3.38545600 & -1.94434600 & 2.96457400\end{array}$ $\begin{array}{lll}-4.47344900 & -2.91365500 & 2.12958000 \\ -1.43261400 & -4.80800400 & -0.64649800\end{array}$ $\begin{array}{lll}-1.43261400 & -4.80800400 & -0.64649800 \\ -1.13832400 & -5.60495300 & -1.35800300\end{array}$ $\begin{array}{lll}-1.13832400 & -5.60495300 & -1.35800300 \\ -2.85942300 & -4.34484300 & -0.98582600\end{array}$ $\begin{array}{lll}-2.85942300 & -4.34484300 & -0.98582600 \\ -2.95321100 & -4.04938500 & -2.05063100\end{array}$ $\begin{array}{lll}-2.95321100 & -4.04938500 & -2.05063100 \\ -3.15543100 & -3.47026300 & -0.37283200\end{array}$ $\begin{array}{lll}-3.15543100 & -3.47026300 & -0.37283200 \\ -3.59028600 & -5.15650600 & -0.80151100\end{array}$ $\begin{array}{rrr}-3.59028600 & -5.15650600 & -0.80151100 \\ 0.12795900 & -4.02293100 & -3.24278800\end{array}$

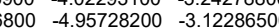
$\begin{array}{lll}-1.10575900 & -4.33669500 & -3.65974000\end{array}$ $\begin{array}{llll}1.5 .57942400 & -3.10683100 & -4.25892100\end{array}$ $\begin{array}{lll}-0.04004300 & -2.14618600 & -4.38568000\end{array}$ $\begin{array}{lll}-1.62356800 & -2.88248000 & -3.96235900\end{array}$ $\begin{array}{lll}-0.62333100 & -3.59970400 & -5.24994200\end{array}$ $\begin{array}{lll}2.82057000 & -2.46893100 & -3.32287200\end{array}$ $\begin{array}{lll}3.43813900 & -3.65136500 & -2.84667100\end{array}$ $\begin{array}{lll}4.15416400 & -4.50333100 & -3.69276000\end{array}$ $\begin{array}{lll}4.63348000 & -5.41355400 & -3.29947000\end{array}$ $\begin{array}{llll}4.25450900 & -4.19272900 & -5.05967300\end{array}$ $\begin{array}{lll}4.81237900 & -4.86455600 & -5.72806600\end{array}$ $\begin{array}{llll}3.63986000 & -3.03482700 & -5.56331000\end{array}$ $\begin{array}{lll}3.70799400 & -2.79531100 & -6.63450300\end{array}$ $2.93913500-2.18082700-4.70086000$

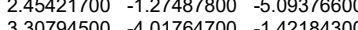
$3.30794500-4.01764700-1.42184300$ $\begin{array}{llll}3.95957300 & -4.78548600 & -1.18676200\end{array}$ $2.33956200-4.35070600-1.22270800$ $3.47678400-3.18508800-0.71576900$ $\begin{array}{lll}5.68673900 & -0.97555400 & -1.96664100\end{array}$ $\begin{array}{llll}6.66682900 & -1.34342300 & -2.32833200\end{array}$ $\begin{array}{llll}5.09384700 & -1.85046600 & -1.64257100\end{array}$ $5.86973000-0.35106300-1.06951800$ $\begin{array}{lll}4.97630800 & -0.18292800 & -3.07546000\end{array}$ $\begin{array}{llll}5.65173900 & 0.62232300 & -3.42485500\end{array}$ $\begin{array}{llll}4.83065700 & -0.84188900 & -3.95201300\end{array}$ $\begin{array}{llll}-3.49470300 & 1.41282000 & -0.90486000\end{array}$ $\begin{array}{lll}1.04827700 & -2.16435300 & -1.61303100\end{array}$ $\begin{array}{lll}-4.05371000 & 2.67257800 & 2.58383700\end{array}$ $\begin{array}{lll}-5.06671200 & 3.36931100 & 3.25387100\end{array}$ $\begin{array}{lll}-6.12692100 & 3.14284400 & 3.06114400\end{array}$ $\begin{array}{rrr}-4.71733600 & 4.36832100 & 4.17635800 \\ -5.50913900 & 4.91586000 & 4.70780100\end{array}$ $\begin{array}{lll}-5.50913900 & 4.91586000 & 4.70780100\end{array}$ $\begin{array}{llll}-3.36470500 & 4.66603900 & 4.41218900\end{array}$ $\begin{array}{lll}-3.09029200 & 5.45109300 & 5.13186500 \\ -4.02519200 & 0.71072300 & 1.74696400\end{array}$ $\begin{array}{lll}-4.02519200 & 0.71072300 & 1.74696400\end{array}$ $\begin{array}{lll}-1.23575100 & -3.71923900 & 2.21462700 \\ -2.62265700 & -3.80805000 & 2.46181300\end{array}$ $\begin{array}{lll}-1.62265700 & -3.80805000 & 2.46181300\end{array}$ $\begin{array}{lll}-0.43853800 & -4.82937600 & 2.56192700 \\ -3.20946800 & -4.95472200 & 3.0065900\end{array}$ \begin{tabular}{rrr}
-1.00614400 & -5.97946800 & 3.12899800 \\
\hline 0.64444000 & -4.77978300 & 2.37686100
\end{tabular} $\begin{array}{rrr}0.64444000 & -4.77978300 & 2.37686100 \\ -2.39327400 & -6.04847000 & 3.33964900\end{array}$ $\begin{array}{lll}-2.39327400 & -6.04847000 & 3.33964900 \\ -4.29562000 & -5.00466600 & 3.18077900\end{array}$ $\begin{array}{lll}-4.29562000 & -5.00466600 & 3.18077900 \\ -0.36678700 & -6.83221300 & 3.39943800 \\ -2.84754400 & -6.95300200 & 3.76940100\end{array}$ $\begin{array}{llll}-0.36678700 & -6.83221300 & 3.39943800 \\ -2.84754400 & -6.95300200 & 3.76940100\end{array}$ $\begin{array}{lll}-2.84754400 & -6.95300200 & 3.76940100 \\ -1.65555100 & -2.93972800 & 4.62946700\end{array}$ $\begin{array}{llll}-1.65555100 & -2.93972800 & 4.62946700 \\ -1.49441600 & -1.55709000 & 5.71243600\end{array}$ $\begin{array}{rrr}-1.49441600 & -1.55709000 & 5.71243600 \\ 0.39483100 & -2.43482600 & 5.10628000\end{array}$ $\begin{array}{lll}0.39483100 & -2.43482600 & 5.10628000 \\ 1.01436300 & -1.55248100 & 5.36057500\end{array}$ $\begin{array}{lll}1.01436300 & -1.55248100 & 5.36057500 \\ 0.43161100 & -3.13740400 & 5.96164600\end{array}$ $\begin{array}{lll}0.43161100 & -3.13740400 & 5.96164600\end{array}$ $\begin{array}{lll}0.85637100 & -2.93953000 & 4.23434600\end{array}$ $\begin{array}{lll}-1.42088600 & -5.28670000 & 0.34716400\end{array}$ $\begin{array}{lll}-2.89886800 & 0.02197500 & -0.88414100\end{array}$ $\begin{array}{lll}-3.36705600 & -0.71081500 & 0.39387900\end{array}$ $\begin{array}{lll}-1.38444700 & 0.02148600 & -0.98792300 \\ -2.55033200 & 1.77089600 & 4.97082800\end{array}$ $\begin{array}{lll}-2.55033200 & 1.77089600 & 4.97082800\end{array}$ $\begin{array}{llll}2.70149900 & 1.39558000 & 1.64875100\end{array}$ $\begin{array}{lll}4.09598000 & -2.02921100 & 1.40539400\end{array}$ $\begin{array}{lll}4.30455500 & -0.86304200 & 2.34681700\end{array}$ $\begin{array}{lll}5.14971900 & -2.70527500 & 3.43223000\end{array}$ $\begin{array}{lll}4.63960300 & -3.27077100 & 2.09013300\end{array}$ $\begin{array}{lll}3.85525900 & -4.05110800 & 2.19198400\end{array}$ $\begin{array}{lll}3.85525900 & -4.05110800 & 2.19198400 \\ 5.44853500 & -3.70800200 & 1.46758500\end{array}$ $\begin{array}{lll}5.68386400 & -1.29298400 & 3.01478000\end{array}$ $\begin{array}{lll}5.68386400 & -1.29298400 & 3.01478000 \\ 3.89496900 & -2.37023700 & 4.26798200\end{array}$ $\begin{array}{lll}3.89496900 & -2.37023700 & 4.26798200 \\ 4.15843200 & -2.10383600 & 5.30855700\end{array}$ $\begin{array}{lll}4.15843200 & -2.10383600 & 5.30855700 \\ 3.20589600 & -3.23535700 & 4.32959600\end{array}$ $\begin{array}{lll}3.20589600 & -3.23535700 & 4.32959600 \\ 5.88879700 & -3.35240200 & 3.93976500\end{array}$ $\begin{array}{lll}5.88879700 & -3.35240200 & 3.93976500 \\ 6.08868400 & -0.40769100 & 4.19936700\end{array}$ $\begin{array}{rrr}6.08868400 & -0.40769100 & 4.19936700 \\ 6.43769400 & 0.58783800 & 3.85640300\end{array}$ $\begin{array}{lll}5.28302100 & -0.24132200 & 4.93843800\end{array}$ 
SUPPORTING INFORIMATION

$\begin{array}{lrrr}\mathrm{H} & 6.94039300 & -0.87301000 & 4.73626200 \\ \mathrm{C} & 6.84460000 & -1.31683600 & 2.00568100 \\ \mathrm{H} & 7.13043900 & -0.28639600 & 1.71109800 \\ \mathrm{H} & 7.74341200 & -1.78134300 & 2.45896500 \\ \mathrm{H} & 6.62612800 & -1.87104400 & 1.07008000 \\ \mathrm{C} & 4.29363600 & 0.52696400 & 1.73389300 \\ \mathrm{H} & 4.65940900 & 0.52914400 & 0.68878600 \\ \mathrm{H} & 4.91865500 & 1.22403700 & 2.32726600 \\ \mathrm{C} & 3.28007700 & -1.16187000 & 3.50712000 \\ \mathrm{H} & 3.18926400 & -0.25187600 & 4.13002300 \\ \mathrm{H} & 2.26597000 & -1.34987500 & 3.10206200 \\ \mathrm{O} & 3.58004200 & -1.98156500 & 0.27770800 \\ \mathrm{C} & 3.18185100 & 7.03704400 & -2.63998500 \\ \mathrm{H} & 2.92421000 & 6.44672400 & -4.71834400 \\ \mathrm{H} & 3.32057500 & 7.35830200 & -0.49270100 \\ \mathrm{H} & 3.62309900 & 8.01483200 & -2.88221200 \\ \mathrm{H} & -3.07157200 & -1.95312500 & -2.06147300\end{array}$

200

\section{Conformer G}

$4.97627900-3.55935300-2.25116000$ $\begin{array}{llll}4.76542200 & -2.02772900 & -2.14822000\end{array}$ $6.67413200-2.31037600-3.36805400$ $\begin{array}{lll}6.24721000 & -3.76326800 & -3.06790800 \\ 6.99214100 & -4.35396200 & -2.49536200\end{array}$ $\begin{array}{lll}6.99214100 & -4.35396200 & -2.49536200 \\ 6.00696300 & -4.35891100 & -3.97287900\end{array}$ $\begin{array}{lll}6.00696300 & -4.35891100 & -3.97287900 \\ 5.30061900 & -1.57262600 & -3.55598100\end{array}$ $\begin{array}{llll}5.30061900 & -1.57262600 & -3.55598100 \\ 7.20002700 & -1.72081200 & -2.03726500\end{array}$ $\begin{array}{lll}7.20002700 & -1.72081200 & -2.03726500 \\ 7.70113300 & -0.74636700 & -2.19286800\end{array}$ $\begin{array}{lll}7.70113300 & -0.74636700 & -2.19286800 \\ 7.94234200 & -2.39021800 & -1.56040500\end{array}$ $\begin{array}{lll}7.94234200 & -2.39021800 & -1.56040500 \\ 7.37156200 & -2.21002900 & -4.22121700\end{array}$ $\begin{array}{lll}7.37156200 & -2.21002900 & -4.22121700 \\ 5.42111100 & -0.05188500 & -3.71894800\end{array}$ $\begin{array}{rrr}5.42111100 & -0.05188500 & -3.71894800 \\ 4.42078100 & 0.42561700 & -3.70013700\end{array}$ $\begin{array}{llll}4.42078100 & 0.42561700 & -3.70013700\end{array}$ $\begin{array}{lll}6.02648600 & 0.43590100 & -2.93261600 \\ 5.88980300 & 0.18777200 & -4.69575200\end{array}$ $\begin{array}{lll}5.88980300 & 0.18777200 & -4.69575200\end{array}$ $\begin{array}{lll}4.44627000 & -2.11385100 & -4.71522400 \\ 3.49308100 & -1.55069000 & -4.80003900\end{array}$ $\begin{array}{llll}3.49308100 & -1.55069000 & -4.80003900 \\ 4.97422500 & -1.98309300 & -5.68164900\end{array}$ $\begin{array}{llll}4.97422500 & -1.98309300 & -5.68164900 \\ 4.18317000 & -3.18712900 & -4.62461600\end{array}$ $\begin{array}{lll}4.32602100 & -1.74066700 & -1.77989600\end{array}$ $2.89161900-2.60790900-1.23955600$ $\begin{array}{lll}2.89161900 & -2.60790900 & -1.23955600 \\ 5.90678000 & -1.56309400 & -1.18155700\end{array}$ $5.90678000-1.56309400-1.18155700$ $\begin{array}{lll}5.73053600 & -0.50660200 & -0.89954000 \\ 5.9163600 & -2.15764000 & -0.24706600\end{array}$ $\begin{array}{llll}5.91636000 & -2.15764000 & -0.24706600\end{array}$ $4.24510600-4.41191800-1.77274700$ $-1.01428300-2.04436900-2.25927100$ $-0.22090400-3.90601400-0.07982800$ $-0.53741900-3.60358400-1.41352900$ $\begin{array}{lll}-1.00464400 & -2.22206100 & -1.43266900\end{array}$ $\begin{array}{lll}-0.49481700 & -2.71495800 & 0.70830900\end{array}$ $\begin{array}{lll}-0.32359300 & -2.53010600 & 2.11159300\end{array}$ $\begin{array}{lll}1.98730300 & 3.87385800 & -0.67236200\end{array}$ $\begin{array}{lll}2.67218200 & 3.46349700 & 0.47576900\end{array}$ $\begin{array}{lll}1.89665400 & 2.37261300 & 1.07635700\end{array}$ $\begin{array}{lll}2.06765400 & 1.73145800 & 2.32372700\end{array}$ $\begin{array}{lll}1.31585400 & 0.60807100 & 2.78927400\end{array}$ $\begin{array}{llll}0.97423700 & 0.23852000 & 4.16065800\end{array}$ $\begin{array}{lll}0.19080100 & -0.92748500 & 4.09137600\end{array}$ $\begin{array}{lll}0.12014500 & -1.31245500 & 2.68680500\end{array}$ $\begin{array}{lll}-2.60512000 & 0.75203500 & -3.31617700\end{array}$ $\begin{array}{lll}-1.64047300 & -0.10246100 & -2.62830000\end{array}$ $\begin{array}{lll}-1.62602100 & -1.51473500 & -2.50399300\end{array}$ $\begin{array}{lll}-0.23212500 & 3.11789800 & -1.77654400\end{array}$ $\begin{array}{lll}-0.70586500 & 0.71691700 & -2.03395500\end{array}$ $\begin{array}{lll}0.16342200 & 0.36150300 & -1.56303900\end{array}$ $\begin{array}{lll}-2.23304800 & 2.07612900 & -3.07725400 \\ -3.86020800 & 0.33195200 & -4.03222700\end{array}$ $\begin{array}{lll}-3.86020800 & 0.33195200 & -4.03222700\end{array}$ $\begin{array}{lll}-3.65228600 & -0.48924300 & -4.74092900\end{array}$ $\begin{array}{lll}-4.20670100 & 1.17820000 & -4.65676400 \\ 0.82824600 & 2.12548700 & 0.23429900\end{array}$ $\begin{array}{lll}0.00919600 & 1.50484700 & 0.44795800\end{array}$ $\begin{array}{rrr}0.00919600 & 1.50484700 & 0.44795800 \\ 0.76291500 & -0.33442800 & 1.95882700\end{array}$ $\begin{array}{lll}1.00399100 & -0.42052200 & 0.92915300\end{array}$ $\begin{array}{lll}1.00399100 & -0.42052200 & 0.92915300\end{array}$ $\begin{array}{rrr}-0.92102000 & -1.73266500 & -0.14938900 \\ -1.33293900 & -0.81689300 & 0.16208400\end{array}$ $\begin{array}{lll}-1.33293900 & -0.81689300 & 0.16208400 \\ -4.99995000 & -0.08297300 & -3.08980500 \\ -5.29223500 & 0.75166600 & -2.42287500\end{array}$ $\begin{array}{lll}-4.99995000 & -0.08297300 & -3.08980500 \\ -5.29223500 & 0.75166600 & -2.42287500\end{array}$ $\begin{array}{lll}-5.29223500 & 0.75166600 & -2.42287500 \\ -4.69716100 & -0.93076900 & -2.44589500\end{array}$ $\begin{array}{lll}-4.69716100 & -0.93076900 & -2.44589500 \\ -5.89389700 & -0.39130400 & -3.66664900\end{array}$ $\begin{array}{lll}-5.89389700 & -0.39130400 & -3.66664900 \\ -2.37936800 & -2.34782700 & -3.47316100\end{array}$ $\begin{array}{lll}-2.37936800 & -2.34782700 & -3.47316100\end{array}$ $\begin{array}{lll}-3.39295400 & -3.23059100 & -3.02928000\end{array}$ $\begin{array}{lll}-4.11858100 & -4.02999300 & -3.91803800 \\ -2.81177900 & -3.11860600 & -5.75786700\end{array}$ $-2.81177900-3.11860600 \quad-5.75786700$ $\begin{array}{llll}-2.10461600 & -2.30989100 & -4.85734400 \\ -1.31144700 & -1.63904400 & -5.21912400\end{array}$ $\begin{array}{lll}-1.31144700 & -1.63904400 & -5.21912400\end{array}$ $\begin{array}{lll}-3.67300400 & -3.35187900 & -1.58140300\end{array}$ $\begin{array}{llll}-3.78016700 & -2.41280100 & -1.58140300 \\ -2.88949600 & -3.85864300 & -1.11289300\end{array}$ $-4.53571400-3.90230600-1.43147900$ $\begin{array}{lll}-0.24159000 & -4.46522700 & -2.61438900\end{array}$ $\begin{array}{lll}-1.13898900 & -4.56291500 & -3.25732800\end{array}$ $\begin{array}{lll}-0.01443700 & -5.49272800 & -2.26678700\end{array}$ $\begin{array}{lll}-1.37585700 & 2.38362400 & 2.49829000\end{array}$ $\begin{array}{rrr}-1.37585700 & 2.38362400 & 2.49829000 \\ 0.93221100 & -3.94401700 & -3.46290000\end{array}$ $\begin{array}{lll}0.76146400 & -2.89951500 & -3.79606200\end{array}$ $\begin{array}{lll}0.76146400 & -2.89951500 & -3.79606200 \\ 1.05810700 & -4.56973100 & -4.36828800\end{array}$ $\begin{array}{lll}1.88223500 & -3.98177600 & -2.89491900\end{array}$ $\begin{array}{lll}1.54141000 & -5.09934200 & 0.42383600\end{array}$ $\begin{array}{llll}0.54141000 & -5.09934200 & 0.42383600 \\ 0.05557600 & -5.51024000 & 1.33006200\end{array}$ $\begin{array}{llll}0.05557600 & -5.51024000 & 1.33006200 \\ 0.50472600 & -5.91279700 & -0.32718000\end{array}$ $\begin{array}{rrr}0.50472600 & -5.91279700 & -0.32718000 \\ -2.76018400 & 2.93108300 & 0.46989900\end{array}$ $\begin{array}{rrr}-2.76018400 & 2.93108300 & 0.46989900 \\ 2.01912400 & -4.75546500 & 0.71506800\end{array}$ $\begin{array}{lll}2.62506800 & -4.66888600 & -0.21040200\end{array}$ $2.49852900-5.53884600 \quad 1.33670400$ $\begin{array}{rrr}2.11794300 & -3.75767700 & 1.20797900 \\ -0.58982700 & -3.70494600 & 2.98461000\end{array}$ $\begin{array}{rrr}-0.58982700 & -3.70494600 & 2.98461000 \\ -1.51000300 & 0.76433800 & 0.59907500\end{array}$ $\begin{array}{lll}-1.51000300 & 0.76433800 & 0.59907500 \\ -1.85120200 & -4.33849200 & 2.95503500\end{array}$ $\begin{array}{lll}-1.85120200 & -4.33849200 & 2.95503500\end{array}$ $\begin{array}{lll}-2.62307800 & -3.93177400 & 2.28478000\end{array}$ $\begin{array}{lll}1.71775900 & -3.63031300 & 3.89150600\end{array}$ $\begin{array}{lll}2.16643900 & -3.72188900 & 2.94954300\end{array}$ $\begin{array}{lll}1.64416400 & -2.60310400 & 4.10836500\end{array}$ $-0.67104700 \quad-1.46032300 \quad 5.21208300$ \begin{tabular}{lll}
-0.36203000 & -0.97472100 & 6.15820000 \\
\hline
\end{tabular} $\begin{array}{lll}-2.16496800 & -1.18469700 & 4.96178000\end{array}$ $\begin{array}{lll}-2.34703900 & -0.09730500 & 4.84641400\end{array}$ $\begin{array}{lll}-2.77609900 & -1.54609100 & 5.81203900\end{array}$ $\begin{array}{rrr}-2.52291500 & -1.70039600 & 4.04673600 \\ 1.17186700 & 1.06469100 & 5.39752000\end{array}$ $\begin{array}{lll}1.17186700 & 1.06469100 & 5.39752000\end{array}$ \begin{tabular}{lll}
4.70175400 & 0.47416200 & 2.62019400 \\
\hline 4.05915900 & 0.16261700 & 1.75716600
\end{tabular} $\begin{array}{rrr}4.05915900 & 0.16261700 & 1.75716600 \\ 4.73487000 & -0.35056600 & 3.24437200\end{array}$ $\begin{array}{rrr}5.65429900 & 0.55794300 & 2.22136200\end{array}$ $\begin{array}{lll}5.65429900 & 0.55794300 & 2.22136200 \\ 4.03663600 & 3.96625600 & 0.85957700\end{array}$ $\begin{array}{llll}4.03663600 & 3.96625600 & 0.85957700 \\ 4.15218000 & 4.99646600 & 0.46814300\end{array}$ $\begin{array}{lll}5.17845900 & 3.09440200 & 0.30451500\end{array}$ $\begin{array}{lll}5.21500500 & 3.12830900 & -0.80102300\end{array}$ $\begin{array}{llll}5.03842300 & 2.02726800 & 0.56154400\end{array}$ $\begin{array}{rrr}6.15463600 & 3.44225900 & 0.69622600 \\ 2.51985400 & 4.81721700 & -1.71411500\end{array}$ $\begin{array}{lll}2.51985400 & 4.81721700 & -1.71411500 \\ 3.35259600 & 5.40135800 & -1.27600300\end{array}$ $\begin{array}{lll}3.35259600 & 5.40135800 & -1.27600300 \\ 1.75645900 & 5.56330900 & -2.00692700 \\ 3.02441500 & 4.06425700 & -2.95945300\end{array}$ $\begin{array}{lll}1.75645900 & 5.56330900 & -2.00692700 \\ 3.02441500 & 4.06425700 & -2.95945300\end{array}$ $\begin{array}{lll}3.02441500 & 4.06425700 & -2.95945300 \\ 2.21596500 & 3.47334500 & -3.43506900\end{array}$ $\begin{array}{llll}3.82921800 & 3.35039600 & -2.69638700\end{array}$ $\begin{array}{llll}3.41730300 & 4.77590600 & -3.71222600\end{array}$ $\begin{array}{llll}-0.51864700 & 4.51185900 & -2.22039900\end{array}$ $\begin{array}{llll}-1.01205900 & 5.44978900 & -1.28397300\end{array}$ $\begin{array}{lll}-1.28419100 & 6.77478500 & -1.64690500\end{array}$ $\begin{array}{llll}-1.67960800 & 7.48749700 & -0.90620300\end{array}$ $\begin{array}{llll}-1.04316800 & 7.19186300 & -2.96539400\end{array}$ $\begin{array}{llll}-1.24813200 & 8.23413800 & -3.25016000\end{array}$ $\begin{array}{llll}-0.53936300 & 6.28179600 & -3.91013800\end{array}$ $\begin{array}{llll}-0.34277700 & 6.60894400 & -4.94156800\end{array}$ $\begin{array}{lll}-0.34277700 & 6.60894400 & -4.94156800 \\ -0.28967900 & 4.95328000 & -3.54078200\end{array}$ $\begin{array}{llll}0.10254200 & 4.23815400 & -4.27852100\end{array}$ $\begin{array}{rrr}0.10254200 & 4.23815400 & -4.27852100 \\ -1.25600400 & 5.02850700 & 0.10711600\end{array}$ $\begin{array}{lll}-1.71939100 & 5.78428800 & 0.63914400\end{array}$ $\begin{array}{lll}-0.36776000 & 4.81809800 & 0.60182600\end{array}$ $\begin{array}{llll}-1.36776000 & 4.81809800 & 0.60182600 \\ -1.89320200 & 4.11058100 & 0.23177900\end{array}$ $\begin{array}{lll}-1.893206100 & 4.79537400 & -2.49224300\end{array}$ $\begin{array}{llll}-4.03582300 & 4.70808300 & -2.87188900\end{array}$ $\begin{array}{lll}-4.53582300 & 4.70808300 & -2.87188900 \\ -3.54728800 & 4.03521900 & -1.53001100\end{array}$ $\begin{array}{lll}-3.54728800 & 4.03521900 & -1.53001100 \\ -4.82512900 & 3.04813300 & -2.27606300\end{array}$ $\begin{array}{llll}-4.82512900 & 3.04813300 & -2.27606300 \\ -3.03426900 & 3.26623700 & -3.53195500\end{array}$ $\begin{array}{lll}-3.03426900 & 3.26623700 & -3.53195500 \\ -3.58671700 & 2.97866700 & -4.44882000 \\ -2.36817300 & 4.08618100 & -3.84455500\end{array}$ $\begin{array}{lll}-3.58671700 & 2.97866700 & -4.44882000 \\ -2.36817300 & 4.08618100 & -3.84455500\end{array}$ $\begin{array}{lll}-2.36817300 & 4.08618100 & -3.84455500 \\ 3.45428100 & 0.93243600 & -1.26055100\end{array}$ $\begin{array}{llll}3.45428100 & 0.93243600 & -1.26055100\end{array}$ $\begin{array}{llll}0.81704600 & 3.01659200 & -0.80688300\end{array}$ $\begin{array}{llll}0.37227900 & -4.24779500 & 3.86532800\end{array}$ $\begin{array}{llll}0.12116700 & -5.35406800 & 4.67897000\end{array}$ $\begin{array}{llll}0.89973700 & -5.75785600 & 5.34494900\end{array}$ $\begin{array}{lll}-1.14926500 & -5.95430800 & 4.63352600\end{array}$ $\begin{array}{lll}-1.36224400 & -6.82302300 & 5.27297500\end{array}$ $\begin{array}{lll}-2.13271100 & -5.44406300 & 3.77190800\end{array}$ $\begin{array}{lll}-3.12688600 & -5.91250600 & 3.73919100\end{array}$ $\begin{array}{llll}2.33145500 & -4.08539400 & 4.59093700\end{array}$ $\begin{array}{lll}3.09899900 & 2.30011000 & 3.24191400\end{array}$ $\begin{array}{lll}3.36455800 & 1.69656900 & 3.37976000\end{array}$ $\begin{array}{llll}2.85480600 & 3.49531000 & 3.95362200\end{array}$ $\begin{array}{lll}5.35677100 & 2.25198000 & 4.20184700\end{array}$ $\begin{array}{lll}5.35677100 & 2.25198000 & 4.20184700 \\ 3.83078700 & 4.05028900 & 4.79011600\end{array}$ $\begin{array}{lll}3.83078700 & 4.05028900 & 4.79011600 \\ 1.87720500 & 3.98458200 & 3.84071000\end{array}$ $5.08554300 \quad 3.42839900 \quad 4.91464900$ $\begin{array}{lll}5.08554300 & 3.42839900 & 4.91464900 \\ 6.34515400 & 1.77367200 & 4.28588000\end{array}$ $\begin{array}{lll}3.61691100 & 4.97790800 & 5.34092800\end{array}$ $5.86168200 \quad 3.86281500 \quad 5.56132200$ $\begin{array}{lll}5.86168200 & 3.86281500 & 5.56132200 \\ 2.17979900 & 1.51828300 & 5.40410100\end{array}$ $\begin{array}{lll}2.17979900 & 1.51828300 & 5.40410100 \\ 1.13638900 & 0.40356800 & 6.28681000\end{array}$ $\begin{array}{lll}1.13638900 & 0.40356800 & 6.28681000 \\ 0.09848200 & 2.16336500 & 5.53245400\end{array}$ $\begin{array}{rrrr}0.09848200 & 2.16336500 & 5.53245400 \\ -0.90337700 & 1.72282300 & 5.70608500\end{array}$ $\begin{array}{rrr}-0.90337700 & 1.72282300 & 5.70608500 \\ 0.32970300 & 2.82669300 & 6.38902600 \\ 0.01664200 & 2.77401900 & 4.61285200\end{array}$ $\begin{array}{lll}0.32970300 & 2.82669300 & 6.38902600 \\ 0.01664200 & 2.77401900 & 4.61285200\end{array}$ $\begin{array}{lll}0.01664200 & 2.77401900 & 4.61285200 \\ 4.13453100 & 4.06113300 & 1.95382700\end{array}$ $\begin{array}{llll}4.13453100 & 4.06113300 & 1.95382700\end{array}$ $\begin{array}{lll}2.94261100 & -0.34694500 & -0.70911200\end{array}$ $\begin{array}{lll}3.47909400 & -0.70104000 & 0.68810800\end{array}$ $\begin{array}{lll}1.40320400 & -0.42075500 & -0.64704700\end{array}$ $\begin{array}{lll}-0.53468800 & -2.54752200 & 5.37849400\end{array}$ $\begin{array}{lll}-2.23172300 & 1.84206200 & 1.40516800\end{array}$ $\begin{array}{lll}-4.77486000 & -0.64537300 & 0.48911100\end{array}$ $\begin{array}{lll}-4.86008600 & 0.59873200 & 1.35926800\end{array}$ $\begin{array}{lll}-7.03414300 & -0.06831100 & 1.00304600\end{array}$ $\begin{array}{lll}-6.19913000 & -1.11526200 & 0.24260700\end{array}$ $\begin{array}{lll}-6.42204800 & -1.17762300 & -0.84317000\end{array}$ $\begin{array}{lll}-6.42552900 & -2.13996800 & 0.65446700\end{array}$ $\begin{array}{lll}-6.13264600 & 0.23116000 & 2.24762600\end{array}$ $\begin{array}{lll}-6.13264600 & 0.23116000 & 2.24762600 \\ -6.94575500 & 1.23809800 & 0.18340800\end{array}$ $\begin{array}{lll}-7.64251300 & 2.00737000 & 0.56543600\end{array}$ $\begin{array}{lll}-7.64251300 & 2.00737000 & 0.565436100 \\ -7.21194600 & 1.07131700 & -0.87910100\end{array}$ $\begin{array}{lll}-8.26124300 & -0.40050100 & 1.24326400\end{array}$ $\begin{array}{lll}-8.64363600 & 1.39351400 & 3.10805000\end{array}$ $\begin{array}{lll}-6.96998600 & 1.58953200 & 3.96708900\end{array}$ $\begin{array}{lll}-6.77045400 & 2.34379100 & 2.55679400\end{array}$ $\begin{array}{lll}-7.63158400 & 1.12850300 \quad 3.53702600\end{array}$ $\begin{array}{lll}-5.87871500 & -0.97625400 & 3.16704400\end{array}$ $\begin{array}{lll}-5.23238300 & -0.69015400 & 4.02201500\end{array}$ $\begin{array}{lll}-6.83113500 & -1.34635700 & 3.59722200\end{array}$ $\begin{array}{lll}-5.38898700 & -1.83627100 & 2.66514100\end{array}$ $\begin{array}{lll}-3.66199100 & 1.03706700 & 2.18325400\end{array}$ $\begin{array}{lll}-3.20054300 & 0.19545800 & 2.73613500\end{array}$ $\begin{array}{lll}-3.98192300 & 1.79472900 & 2.92687500\end{array}$ $\begin{array}{lll}-5.46373700 & 1.66559900 & 0.37058000\end{array}$ $\begin{array}{lll}-5.35647900 & 2.66360200 & 0.83669500\end{array}$ $\begin{array}{llll}-4.87821400 & 1.71323200 & -0.56608500\end{array}$ $\begin{array}{lll}-3.75302600 & -1.18781900 & 0.03884600\end{array}$ $\begin{array}{lll}-3.82495900 & -3.97223800 & -5.29093900\end{array}$ $\begin{array}{lll}-2.57346200 & -3.08134000 & -6.83089800\end{array}$ $\begin{array}{lll}-4.90773800 & -4.70590700 & -3.55286800\end{array}$ $\begin{array}{llll}-4.38971700 & -4.60351000 & -5.99225500\end{array}$

$\begin{array}{llll}2.69917200 & -1.55318500 & -2.67433700\end{array}$

\section{Conformer $\mathbf{H}$}

$\begin{array}{lll}2.83701100 & -3.38087900 & 1.95664100\end{array}$ \begin{tabular}{lll}
3.68969000 & -2.49698200 & 2.86533800 \\
\hline 4.85429700 & -4.45887400 & 2.67207100
\end{tabular} $\begin{array}{lll}4.85429700 & -4.45887400 & 2.67207100\end{array}$ $\begin{array}{lll}3.63035900 & -4.66774100 & 1.75254600 \\ 3.01423400 & -5.55763000 & 1.99647500\end{array}$ $3.01423400-5.55763000-1.99647500$ $\begin{array}{llll}3.90394500 & -4.75739400 & 0.68056200 \\ 5.16419000 & -2.93238000 & 2.48573900\end{array}$ $\begin{array}{llll}5.16419000 & -2.93238000 & 2.48573900\end{array}$ $\begin{array}{lll}4.33943600 & -4.53772600 & 4.12492500 \\ 5.17251200 & -4.56706700 & 4.85253400\end{array}$ $\begin{array}{llll}5.17251200 & -4.56706700 & 4.85253400\end{array}$ $\begin{array}{lll}3.72985000 & -5.44623100 & 4.29894400\end{array}$ $\begin{array}{llll}5.70603400 & -5.12778400 & 2.44450900\end{array}$ $\begin{array}{lll}6.22539600 & -2.38561700 & 3.45164000\end{array}$ $\begin{array}{lll}6.37614900 & -1.29687300 & 3.29626800\end{array}$ $\begin{array}{llll}5.99711100 & -2.54017000 & 4.52289300\end{array}$ $\begin{array}{llll}7.20284600 & -2.87078900 & 3.25194300\end{array}$ $\begin{array}{lll}5.61822600 & -2.59740000 & 1.05755300\end{array}$ $\begin{array}{lll}5.82999100 & -1.51684500 & 0.95295100\end{array}$ $\begin{array}{lll}6.55257700 & -3.15020500 & 0.82778500\end{array}$ $\begin{array}{lll}4.86989800 & -2.84500200 & 0.28396100\end{array}$ $\begin{array}{lll}3.30514500 & -1.03190200 & 3.00404800\end{array}$ $\begin{array}{llll}4.13041900 & -0.45630900 & 3.46692500\end{array}$ $\begin{array}{lll}3.50895400 & -3.23216000 & 4.25808700\end{array}$ $3.88838800-256946900 \quad 5.06171200$ $\begin{array}{lll}3.88838800 & -2.56946900 & 5.06171200 \\ 2.43881800 & -3.41123700 & 4.47474500\end{array}$ $\begin{array}{rrr}2.43881800 & -3.41123700 & 4.47474500 \\ 1.70278400 & -3.13875800 & 1.55523000\end{array}$ $\begin{array}{lll}1.70278400 & -3.13875800 & 1.55523000 \\ 0.36241600 & -2.02496300 & -1.88454800\end{array}$ $\begin{array}{lll}0.36241600 & -2.02496300 & -1.88454800\end{array}$ $\begin{array}{lll}-1.83781200 & 0.26571800 & 3.71030000 \\ -1.70303600 & -1.10638700 & 3.45409900\end{array}$ $-1.43111000-1.25398000 \quad 2.02385600$ $\begin{array}{lll}-1.60640600 & 0.95965200 & 2.45663100\end{array}$ $\begin{array}{lll}-1.58048800 & 2.36598500 & 2.22345800\end{array}$ $\begin{array}{lll}3.47031100 & 0.10440800 & -2.69033900 \\ 3.79498300 & 1.30561200 & -2.05291500\end{array}$ $\begin{array}{llll}3.79498300 & 1.30561200 & -2.05291500\end{array}$ $\begin{array}{llll}2.59844100 & 1.74983400 & -1.33470400\end{array}$ $\begin{array}{llll}2.33595600 & 2.98887100 & -0.71404700\end{array}$ $\begin{array}{llll}1.14480400 & 3.30137400 & 0.02628300\end{array}$ $\begin{array}{llll}0.36556500 & 4.52955700 & -0.03843800\end{array}$ $\begin{array}{llll}-0.81098600 & 4.31366100 & 0.70313800\end{array}$ $\begin{array}{lll}-0.71131800 & 2.98392500 & 1.28491000\end{array}$ $\begin{array}{lll}-1.20884700 & -3.51875500 & -1.09328200\end{array}$ $\begin{array}{lll}-0.85297100 & -2.56140200 & -0.05189000\end{array}$ $\begin{array}{lll}-1.38660400 & -2.44999000 & 1.26079100\end{array}$ $\begin{array}{lll}1.35528100 & -1.39597400 & -2.66746700\end{array}$ $\begin{array}{llll}0.08684900 & -1.69560900 & -0.56721300\end{array}$ $\begin{array}{llll}0.69051700 & -1.10766200 & 0.06531300\end{array}$ $\begin{array}{llll}-0.50198400 & -3.15648100 & -2.24246500\end{array}$ $\begin{array}{lll}-2.51312600 & -4.64067100 & -1.03528800\end{array}$ $\begin{array}{lll}-2.21312600 & -4.64067100 & -1.03528800 \\ -2.21455400 & -5.10993600 & -0.03848900\end{array}$ $\begin{array}{lll}-1.87083000 & -5.43921900 & -1.03848900 \\ -1.72417400\end{array}$ $\begin{array}{ccc}-1.87083000 & -5.43921900 & -1.72417400 \\ 1.64090600 & 0.75896400 & -1.47589600\end{array}$ $\begin{array}{lll}1.64090600 & 0.75896400 & -1.47589600\end{array}$ $\begin{array}{lll}0.61238200 & 0.88505800 & -1.25997600\end{array}$ $\begin{array}{lll}0.46714000 & 2.41850300 \quad 0.83401600\end{array}$ $\begin{array}{lll}0.83714600 & 1.46108200 & 1.11199800\end{array}$ $\begin{array}{lll}-1.31864400 & 0.27139800 \quad 0.47681600\end{array}$ $-1.218644000 .271398000 .47681600$ $\begin{array}{lll}-3.64772300 & -4.24324600 & -1.41225400\end{array}$ $\begin{array}{lll}-3.70934000 & -3.89471200 & -2.46234400\end{array}$ $-4.032543000-3.42691900-0.77076400$ $-4.32936200-5.11059400-1.30968800$ $\begin{array}{lll}-1.93747700 & -3.68861700 & 1.87137600\end{array}$ $\begin{array}{llll}-3.28617500 & -3.81651700 & 2.27037400\end{array}$ $\begin{array}{lll}-3.78698200 & -5.00667400 & 2.80759000\end{array}$ $\begin{array}{lll}-1.56962100 & -5.99085200 & 2.61529300\end{array}$ $\begin{array}{lll}-1.08453200 & -4.79820700 & 2.06025100\end{array}$ $\begin{array}{lll}-0.03091800 & -4.68609900 & 1.75840900\end{array}$ $\begin{array}{lll}-4.19007700 & -2.65061400 & 2.17975300\end{array}$ $\begin{array}{lll}-4.39957700 & -2.27615400 & 1.19981800\end{array}$ $\begin{array}{lll}-3.79057400 & -1.84753600 & 2.71467900\end{array}$ $\begin{array}{lll}-5.11034400 & -2.87587700 & 2.59633100\end{array}$ $\begin{array}{lll}-1.54790200 & -2.16628600 & 4.51557400\end{array}$ $-2.15382600-3.06735500 \quad 4.30432200$ $\begin{array}{lll}-1.92887300 & -1.77087100 & 5.47756900\end{array}$ $\begin{array}{lll}-2.17214200 & 2.29235900 & -2.81043200 \\ -0.07390500 & -2.58136700 & 4.66951100\end{array}$ $\begin{array}{lll}-0.07390500 & -2.58136700 & 4.66951100\end{array}$ $0.34983300-2.91058400 \quad 3.70017900$ $0.02519500-3.40613600-5.40240900$ $\begin{array}{lll}0.53704800 & -1.73001700 & 5.03289800 \\ -1.90283600 & 0.92211200 & 5.06186800\end{array}$ $\begin{array}{lll}-1.90283600 & 0.92211200 & 5.06186800\end{array}$ $\begin{array}{ll}-2.6846800 & 1.70533100 \quad 5.07905000\end{array}$ $\begin{array}{lll}-2.21268600 & 0.17802100 & 5.82158800\end{array}$ $\begin{array}{lll}-1.51988000 & -0.12222400 & -3.16239900\end{array}$ $\begin{array}{lll}-0.54789600 & 1.52405000 & 5.47593900 \\ 0.22330700 & 0.74019600 & 5.61168800\end{array}$ $\begin{array}{rrr}0.22330700 & 0.74019600 & 5.61168800 \\ -0.62889300 & 2.08530100 & 6.42724800\end{array}$ 
SUPPORTING INFORMATION

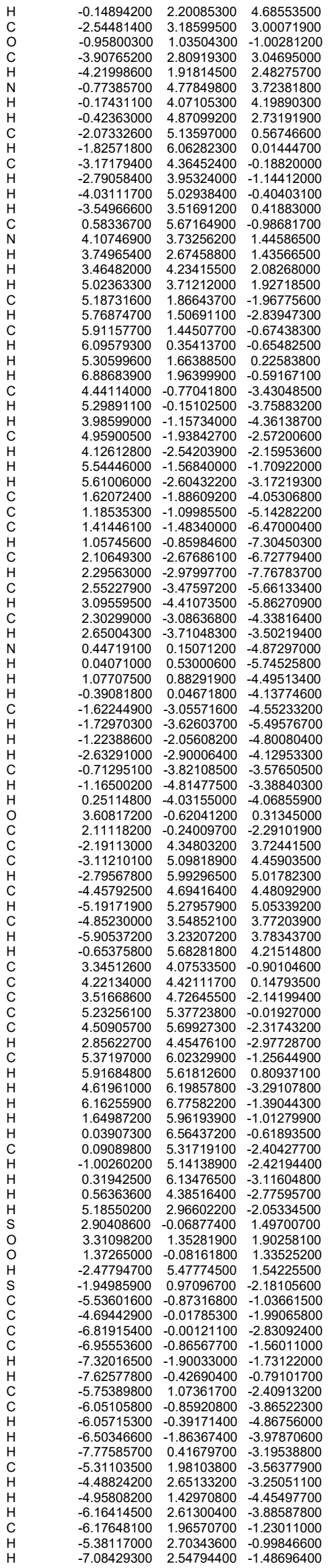

$\begin{array}{lll}-6.39638400 & 1.41245400 & -0.29354000\end{array}$ $\begin{array}{lll}-3.40720800 & 0.35613800 & -1.29326600\end{array}$ $\begin{array}{lll}-3.01668300 & -0.53329000 & -0.75625600\end{array}$ $\begin{array}{lll}-3.58159100 & 1.15232200 & -0.54026100\end{array}$ $\begin{array}{lll}-4.61259200 & -0.91773300 & -3.27497400\end{array}$ $\begin{array}{lll}-3.84867300 & -0.49281200 & -3.95264700\end{array}$ $\begin{array}{lll}-4.27405100 & -1.94161700 & -3.02556000\end{array}$ $\begin{array}{lll}-5.11194400 & -1.46028700 & -0.03609600\end{array}$ $\begin{array}{lll}-2.92236100 & -6.10204800 & 2.97603400\end{array}$ $\begin{array}{lll}-0.89086500 & -6.84416100 & 2.75953000\end{array}$ $\begin{array}{lll}-4.84405000 & -5.08842400 & 3.10579500\end{array}$ $\begin{array}{llll}-3.31295400 & -7.03869000 & 3.39970500\end{array}$ $\begin{array}{lll}2.40018300 & -0.89907300 & 3.62910600\end{array}$

138

\section{$\left[\alpha_{2} \beta_{2}-\boldsymbol{P}\right]^{4+}$ conf. 1}

$\begin{array}{llll}C & -1.30026300 & 2.86766700 & -0.27331600\end{array}$ $\begin{array}{lll}4.11011300 & -0.35962700 & 1.05008400\end{array}$ $\begin{array}{rrr}3.99713900 & 1.02501600 & 1.11362300\end{array}$ $\begin{array}{lll}3.99713900 & 1.02501600 & 1.11362300 \\ 2.68778900 & 1.34235100 & 0.48946800\end{array}$ $\begin{array}{lll}2.84836800 & -0.84370600 & 0.42863900\end{array}$ $\begin{array}{lll}2.84836800 & -0.84370600 & 0.42863900 \\ 2.57361100 & -2.19210600 & 0.03443100\end{array}$ $\begin{array}{rrr}2.57361100 & -2.19210600 & 0.03443100 \\ -4.07629000 & 0.56432300 & 1.17840700\end{array}$ $\begin{array}{lll}-4.07317500 & -0.81996000 & 1.04669100\end{array}$ $\begin{array}{lll}-4.04317500 & -0.81996000 & 1.04669100 \\ -2.75160100 & -1.13394900 & 0.37874300\end{array}$ $\begin{array}{llll}-2.75160100 & -1.13394900 & 0.37874300 \\ -2.33639500 & -2.42773000 & -0.07045300\end{array}$ $\begin{array}{lll}-2.33639500 & -2.42773000 & -0.07045300 \\ -0.99596600 & -2.79311000 & -0.37142100\end{array}$ $\begin{array}{lll}-0.99596600 & -2.79311000 & -0.37142100 \\ -0.48645800 & -4.04287500 & -0.93399000\end{array}$ $\begin{array}{rrr}-0.48645800 & -4.04287500 & -0.93399000 \\ 0.91774000 & -3.99393100 & -0.86983900\end{array}$ $\begin{array}{llll}0.91774000 & -3.99393100 & -0.86983900 \\ 1.28657800 & -2.70043100 & -0.29572000\end{array}$ $\begin{array}{lll}1.28657800 & -2.70043100 & -0.29572000 \\ 0.45414800 & 4.14314200 & -1.04216900\end{array}$ $\begin{array}{llll}0.45414800 & 4.14314200 & -1.04216900\end{array}$ $\begin{array}{lll}0.96102700 & 2.97588000 & -0.32868300\end{array}$ $\begin{array}{lll}2.28118900 & 2.64261900 & 0.06373900\end{array}$ $\begin{array}{rrrr}-2.56590100 & 2.39771400 & 0.16059400\end{array}$ $\begin{array}{lll}-0.12816400 & 2.21605600 & 0.02822500\end{array}$ $\begin{array}{lll}-0.07288300 & 1.29264900 & 0.47130700\end{array}$ $\begin{array}{rrr}-0.94350200 & 4.07903700 & -1.00211400\end{array}$ $\begin{array}{lll}1.25920400 & 5.11245400 & -1.86546000\end{array}$ $\begin{array}{llll}2.06055500 & 5.58086400 & -1.26486100\end{array}$ $\begin{array}{llll}0.60305600 & 5.94809500 & -2.17862900\end{array}$ $\begin{array}{lll}-2.02122000 & 0.00074700 & 0.16576400\end{array}$ $\begin{array}{lll}-2.10633100 & -2.02054800 & -0.05511200\end{array}$ $\begin{array}{llll}0.05900500 & -1.02505800 & 0.20600900\end{array}$ $\begin{array}{rrr}1.99703300 & 0.19800200 & 0.17713000\end{array}$ $\begin{array}{llll}1.86467300 & 4.45925600 & -3.11900800\end{array}$ $\begin{array}{lll}1.86467300 & 4.45925600 & -3.11900800 \\ 1.08214200 & 4.05357800 & -3.79064600\end{array}$ $\begin{array}{llll}2.52664800 & 3.60661800 & -2.85346300\end{array}$ $\begin{array}{llll}2.52664800 & 3.60661800 & -2.85346300 \\ 2.46957000 & 5.18689500 & -3.69491800\end{array}$ $\begin{array}{rrr}2.469510200 & 3.72162100 & 0.01823600\end{array}$ $\begin{array}{llr}3.30810200 & 3.72162100 & 0.01823600 \\ 4.46528000 & 3.60537700 & -0.78111600\end{array}$ $\begin{array}{lll}4.46528000 & 3.60537700 & -0.78111600 \\ 5.44854600 & 4.59483300 & -0.85048000\end{array}$ $\begin{array}{llr}5.44854600 & 4.59483300 & -0.85048000 \\ 4.16139800 & 5.89932400 & 0.74861600\end{array}$ $\begin{array}{lll}4.16139800 & 5.89932400 & 0.74861600 \\ 3.17892500 & 4.89832000 & 0.78575600\end{array}$ $\begin{array}{lll}3.17892500 & 4.89832000 & 0.78575600 \\ 2.29157000 & 5.01642200 & 1.42499000\end{array}$ $\begin{array}{rrr}2.29157000 & 5.01642200 & 1.42499000 \\ 4.66071400 & 2.34354600 & -1.53427800\end{array}$ $\begin{array}{llll}3.85756900 & 2.15122800 & -2.16761000\end{array}$ $\begin{array}{llll}4.70737400 & 1.53387900 & -0.85535400\end{array}$ $\begin{array}{llll}5.52463100 & 2.36736200 & -2.10468100\end{array}$ $\begin{array}{llll}4.86517800 & 1.93198500 & 1.95757600\end{array}$ $\begin{array}{lll}5.25989700 & 2.79829400 & 1.39203400 \\ 5.76189100 & 1.36452700 & 2.27824700\end{array}$ $\begin{array}{lll}5.76189100 & 1.36452700 & 2.27824700\end{array}$ $\begin{array}{lll}4.11961300 & 2.44872700 & 3.20116200\end{array}$ $\begin{array}{lll}3.23245900 & 3.05351400 & 2.92637300 \\ 4.78538800 & 3.08654500 & 3.81581300\end{array}$ $\begin{array}{llll}3.76495200 & 1.61227800 & 3.83649700\end{array}$ $\begin{array}{llll}5.17251200 & -1.16465800 & 1.74996400\end{array}$ $\begin{array}{lll}5.50273700 & -2.02262100 & 1.13864300\end{array}$ $\begin{array}{lll}5.07976700 & -0.54198300 & 1.88266600\end{array}$ $\begin{array}{lll}4.70527400 & -1.64232700 & 3.14023400\end{array}$ $\begin{array}{lll}4.76527400 & -1.64232700 & 3.14023400 \\ 4.66123900 & -0.80658900 & 3.86648300\end{array}$ $\begin{array}{lll}5.38300900 & -2.41084400 & 3.56335700\end{array}$ $\begin{array}{lll}5.38300900 & -2.41084400 & 3.56335700 \\ 3.65825400 & -2.02917700 & 3.11814700\end{array}$ $\begin{array}{lll}3.76057500 & -3.09008000 & -0.14489800\end{array}$ $\begin{array}{lll}3.765214200 & -2.88178000 & -1.21648600\end{array}$ $\begin{array}{lll}4.65214200 & -2.88178000 & -1.21648600 \\ 4.44247900 & -2.06483900 & -1.92157400\end{array}$ $\begin{array}{rrr}4.44247900 & -2.06483900 & -1.92157400 \\ 3.16703600 & -4.36050700 & 1.89053900\end{array}$ $\begin{array}{lll}3.16703600 & -4.36050700 & 1.89053900 \\ 2.17346400 & -4.28770000 & 1.58937500\end{array}$ $\begin{array}{lll}2.17346400 & -4.28770000 & 1.58937500 \\ 3.31558100 & -5.28347700 & 2.33820800\end{array}$ $\begin{array}{rrr}3.31558100 & -5.28347700 & 2.33820800 \\ 1.81972200 & -5.01144800 & -1.52420400\end{array}$ $\begin{array}{lll}1.81972200 & -5.01144800 & -1.52420400 \\ 1.29107800 & -5.98462800 & -1.56117900\end{array}$

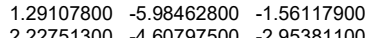
$\begin{array}{lll}2.22751300 & -4.60797500 & -2.95381100 \\ 1.34374400 & -4.52573400 & -3.61626600\end{array}$ $\begin{array}{lll}1.34374400 & -4.52573400 & -3.61626600\end{array}$ $\begin{array}{llll}2.90981000 & -5.36450100 & -3.38919400\end{array}$ $\begin{array}{lll}2.74528000 & -3.62917300 & -2.97138400\end{array}$ $\begin{array}{lll}-1.25264800 & -5.07707900 & -1.72545000\end{array}$ $\begin{array}{lll}-2.58592300 & -4.82996900 & 1.56614900\end{array}$ $\begin{array}{lll}-1.61603900 & -4.67062200 & 1.21217300\end{array}$ $\begin{array}{lll}-2.65037300 & -5.79110800 & 1.94789200\end{array}$ $\begin{array}{lll}-2.75056100 & -4.15818200 & 2.35086700\end{array}$ $\begin{array}{lll}-4.98541300 & -1.77628900 & 1.72710800\end{array}$ $\begin{array}{lll}-5.93663400 & -1.25659600 & 1.95808400\end{array}$ $\begin{array}{lll}-4.39615300 & -2.31802000 & 3.04446900\end{array}$ $\begin{array}{lll}-4.35689800 & -1.53539200 & 3.82787900\end{array}$ $\begin{array}{lll}-3.33298000 & -2.63357800 & 2.92115300\end{array}$ $\begin{array}{rrr}-5.33298000 & -2.63357800 & 2.92115300 \\ -5.01103300 & 1.33493000 & 2.08404600\end{array}$ $\begin{array}{lll}-5.01103300 & 1.33493000 & 2.08404600 \\ -5.83851600 & 0.66434300 & 2.39175800\end{array}$ $\begin{array}{lll}-5.83851600 & 0.66434300 & 2.39175800 \\ -5.50252000 & 2.18467800 & 1.57126000\end{array}$ $\begin{array}{llll}-5.50252000 & 2.18467800 & 1.57126000 \\ -4.29115100 & 1.86327000 & 3.33787400\end{array}$ $\begin{array}{lll}-4.29115100 & 1.86327000 & 3.33787400 \\ -3.47905000 & 2.57156600 & 3.07835900\end{array}$ $\begin{array}{lll}-3.4753593100 & 1.03670900 & 3.91966400 \\ -3.8359\end{array}$ $\begin{array}{lll}-3.83593100 & 1.03670900 & 3.91966400 \\ -5.00357500 & 2.39519500 & 3.99913700\end{array}$ $\begin{array}{lll}-3.69000600 & 3.37516700 & 0.19335900\end{array}$ $\begin{array}{lll}-4.86345500 & 3.18102600 & -0.56626000\end{array}$ $\begin{array}{llll}-5.93185400 & 4.08063300 & -0.56361100\end{array}$ $\begin{array}{llll}-6.82937100 & 3.90004300 & -1.17550600\end{array}$ $\begin{array}{llll}-5.84623200 & 5.22515400 & 0.24722800\end{array}$ $\begin{array}{lll}-6.68080300 & 5.94089100 & 0.26430000\end{array}$ $\begin{array}{lll}-4.70182000 & 5.44388700 & 1.03120000\end{array}$ $\begin{array}{lll}-4.63775300 & 6.33479000 & 1.67304100\end{array}$ $\begin{array}{lll}-3.63434800 & 4.53396300 & 0.99625200\end{array}$ $\begin{array}{llll}-2.73456700 & 4.71176600 & 1.60330400\end{array}$ $\begin{array}{lll}-4.97520500 & 1.93492600 & -1.36095900\end{array}$ $\begin{array}{llll}-5.86708700 & 1.89450300 & -1.88558400\end{array}$ $\begin{array}{llll}-4.90549500 & 1.09870900 & -0.71585400\end{array}$ $\begin{array}{llll}-4.19300200 & 1.84890600 & -2.04239100\end{array}$ $\begin{array}{llll}-2.46551800 & 4.28761000 & -3.01348900\end{array}$ $\begin{array}{llll}-2.46551800 & 4.28761000 & -3.01348900 \\ -3.17778300 & 4.95343900 & -3.53928500\end{array}$ $\begin{array}{lll}-3.100806200 & 3.35418300 & -2.54711900\end{array}$ $\begin{array}{lll}-3.00806200 & 3.35418300 & -2.74711900 \\ -1.67369400 & 3.99497200 & -3.73130500\end{array}$ $\begin{array}{lll}-1.67369400 & 3.99497200 & -3.73130500 \\ -1.88137300 & 4.97366700 & -1.76742800\end{array}$ $\begin{array}{lll}-1.88137300 & 4.97366700 & -1.76742800 \\ -1.33324000 & 5.88295600 & -2.08333100\end{array}$ $\begin{array}{llll}-1.33324000 & 5.88295600 & -2.08333100 \\ -2.70141600 & 5.33849600 & -1.12200800\end{array}$ $\begin{array}{lll}-2.70141600 & 5.33849600 & -1.12200800 \\ -2.82331800 & 1.04912600 & 0.54464800\end{array}$ $\begin{array}{lll}-2.82331800 & 1.04912600 & 0.54464800 \\ 4.06827100 & -4.14745600 & 0.73426800\end{array}$ $\begin{array}{lll}4.06827100 & -4.14745600 & 0.73426800\end{array}$ $\begin{array}{llll}5.18967700 & -4.96776400 & 0.59075500 \\ 5.39794500 & -5.77956100 & 1.30477600\end{array}$ $\begin{array}{lll}5.39794500 & -5.77956100 & -1.30477600\end{array}$ $\begin{array}{lll}6.05459500 & -4.73698500 & -0.49226700 \\ 6.94007300 & -5.37507800 & -0.62530100\end{array}$ $\begin{array}{llll}6.94007300 & -5.37507800 & -0.62530100\end{array}$ $\begin{array}{lll}5.78259800 & -3.69535000 & -1.39300900\end{array}$ $\begin{array}{lll}6.45813200 & -3.51496800 & -2.24192800\end{array}$ $\begin{array}{lll}3.32731700 & -3.61957500 & 2.61390500\end{array}$ $\begin{array}{lll}-3.42078300 & -3.43443200 & -0.29734100\end{array}$ $\begin{array}{lll}-3.56939200 & -4.59867900 & 0.48283200\end{array}$ $-4.37820400-3.23210700-1.31288300$ $\begin{array}{lll}-4.60282700 & -5.52100900 & 0.30251200\end{array}$ $\begin{array}{lll}-5.41994700 & -4.14802500 & -1.52788000\end{array}$ $\begin{array}{lll}-4.29022400 & -2.33611600 & -1.94385200\end{array}$ $\begin{array}{lll}-5.53811400 & -5.29000600 & -0.72016300\end{array}$ $\begin{array}{lll}-4.68757800 & -6.41376800 & 0.94162000\end{array}$ $\begin{array}{lll}-6.14713100 & -3.96945000 & -2.33345400\end{array}$ $\begin{array}{lll}-6.35540000 & -6.00763700 & -0.88131600\end{array}$ $\begin{array}{llll}-2.14333300 & -5.45347800 & -1.18919200\end{array}$ $\begin{array}{lll}-2.1433300 & -5.45347800 & -1.18919200 \\ -1.60474600 & -5.96338200 & -1.87067300\end{array}$ $\begin{array}{lll}-1.69286900 & -4.54603600 & -3.870309600\end{array}$ $\begin{array}{lll}-1.69286900 & -4.54603600 & -3.10309600 \\ -0.82039200 & -4.24232300 & -3.71429200\end{array}$ $\begin{array}{lll}-0.82039200 & -4.24232300 & -3.71429200\end{array}$ $\begin{array}{lll}-2.24503900 & -5.32879300 & -3.65982300\end{array}$ $-2.35403100-3.66246900-3.01158200$ $\begin{array}{lll}-5.26779900 & -2.61185800 & 1.06211700 \\ 2.73184100 & -5.19933100 & -0.03065200\end{array}$ $2.73184100-5.19933100-0.93065200$

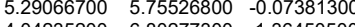
$\begin{array}{lll}4.04235200 & 6.80277300 & 1.36450500\end{array}$ $6.33593700-4.47191300-1.49081500$ $\begin{array}{rrr}6.05796700 & 6.54193800 & -0.11260000 \\ -4.99197600 & -3.16218400 & 3.44553700\end{array}$

138

$\left[\alpha_{2} \beta_{2}-P\right]^{4+}$ conf. 2

\begin{tabular}{lrrr}
$\mathrm{C}$ & -2.62587300 & 1.62295600 & -0.29829800 \\
$\mathrm{C}$ & 3.63861700 & 1.97986200 & 0.95672700 \\
$\mathrm{C}$ & 2.77335100 & 3.08530600 & 0.91992400 \\
$\mathrm{C}$ & 1.51277800 & 2.62889800 & 0.34095600 \\
$\mathrm{C}$ & 2.90813700 & 0.83046300 & 0.43043700 \\
$\mathrm{C}$ & 3.37855500 & -0.47883200 & 0.13234700 \\
$\mathrm{C}$ & -3.68755400 & -1.83922000 & 1.13556300 \\
$\mathrm{C}$ & -2.88249000 & -2.97574900 & 0.94638700 \\
$\mathrm{C}$ & -1.66547900 & -2.54360900 & 0.26442300 \\
$\mathrm{C}$ & -0.57069700 & -3.32426300 & -0.19930100 \\
$\mathrm{C}$ & 0.74201800 & -2.82480700 & -0.46285200 \\
$\mathrm{C}$ & 1.85421600 & -3.62509100 & -1.04007000 \\
$\mathrm{C}$ & 3.00548900 & -2.86180900 & -0.85124300 \\
$\mathrm{C}$ & 2.54780300 & -1.58724800 & -0.23377600 \\
$\mathrm{C}$ & -1.95075900 & 3.62892400 & -1.15717000 \\
$\mathrm{C}$ & -0.85718200 & 2.88274400 & -0.48067300 \\
$\mathrm{C}$ & 0.41107800 & 3.40825500 & -0.10686900 \\
$\mathrm{C}$ & -3.46243200 & 0.58533800 & 0.20967400 \\
$\mathrm{~N}$ & -1.27369100 & 1.63710900 & -0.08729800 \\
$\mathrm{C}$ & -3.07653100 & 2.83902800 & -1.02031400 \\
$\mathrm{C}$ & -1.82896800 & 4.85103000 & -2.02778700 \\
$\mathrm{H}$ & -1.39921900 & 5.70726800 & -1.47521200 \\
$\mathrm{H}$ & -2.84325800 & 5.18160800 & -2.32911000 \\
$\mathrm{~N}$ & -1.76315400 & -1.18216700 & 0.10047000 \\
$\mathrm{~N}$ & 1.18679100 & -1.59537000 & -0.04919100 \\
$\mathrm{~N}$ & 1.63160600 & 1.26904300 & 0.14419500 \\
$\mathrm{C}$ & -1.00291300 & 4.58524900 & -3.29755100 \\
$\mathrm{H}$ & -1.48347300 & 3.83016000 & -3.95082500 \\
$\mathrm{H}$ & 0.00216300 & 4.17245800 & -3.05287400 \\
$\mathrm{H}$ & -0.85947700 & 5.51070100 & -3.88945000 \\
$\mathrm{C}$ & 0.57578200 & 4.89226000 & -0.12653500 \\
$\mathrm{C}$ & 1.50449200 & 5.53928000 & -0.96706100 \\
$\mathrm{C}$ & 1.65762200 & 6.92712100 & -1.01463200 \\
$\mathrm{C}$ & -0.06288400 & 7.11170900 & 0.69365600 \\
$\mathrm{C}$ & -0.20773100 & 5.71613100 & 0.70853000 \\
$\mathrm{H}$ & -0.94264800 & 5.24108200 & 1.37460300 \\
$\mathrm{~N}$ & 2.37272200 & 4.69214400 & -1.81994000 \\
$\mathrm{H}$ & 1.80513200 & 4.15463100 & -2.51007600 \\
$\mathrm{H}$ & 2.88750000 & 3.99236100 & -1.23174900 \\
$\mathrm{H}$ & 3.06217800 & 5.25790300 & -2.34738500 \\
$\mathrm{C}$ & 3.02794400 & 4.38597900 & 1.64731400 \\
$\mathrm{H}$ & 2.81359000 & 5.27534000 & 1.02529800 \\
$\mathrm{H}$ & 4.10800800 & 4.45154600 & 1.88487700 \\
$\mathrm{C}$ & 2.21229800 & 4.48413800 & 2.94990100 \\
$\mathrm{H}$ & 1.12257900 & 4.44795500 & 2.75390100 \\
$\mathrm{H}$ & 2.43255000 & 5.43504000 & 3.47417400 \\
$\mathrm{H}$ & 2.44950300 & 3.64940700 & 3.63930400 \\
& & & \\
\hline
\end{tabular}




\section{SUPPORTING INFORVATION}

$\begin{array}{llll}\text { C } & 4.97782400 & 1.97988700 & 1.64628800\end{array}$

$\begin{array}{llll}\mathrm{H} & 5.70415700 & 1.34479800 & 1.11218300\end{array}$

H $\quad 5.40854500 \quad 3.00002900 \quad 1.60900500$

$\begin{array}{llll}\mathrm{C} & 4.87531200 & 1.54814400 & 3.12323800 \\ \mathrm{H} & 4.40524300 & 2.33093900 & 3.75060000\end{array}$

$\begin{array}{lll}4.40524300 & 2.33093900 & 3.75060000 \\ 5.87241400 & 1.33261600 & 3.55612100\end{array}$

$\begin{array}{lll}4.21023700 & 0.66213500 & 3.25305800\end{array}$

$\begin{array}{lll}4.86831700 & -0.64115200 & 0.08660100\end{array}$

$\begin{array}{lll}5.62050800 & -0.12443000 & -0.98807400\end{array}$

$\begin{array}{lll}5.09313300 & 0.40388900 & -1.79452000 \\ 4.82059200 & -1.76274400 & 2.28610100\end{array}$

$\begin{array}{lll}3.86909200 & -2.06582700 & 1.99127100\end{array}$

$\begin{array}{lll}5.29385400 & -2.54195700 & 2.77872200\end{array}$

$4.35181100-3.22528700-1.42402200$

$4.44158900-4.33060200-1.44268900$

$\begin{array}{lll}4.55019600 & -2.68852700 & -2.85468600 \\ 3.83104900 & -3.14901300 & -3.56059000\end{array}$

$3.83104900-3.14901300-3.56059000$

$5.57276900-2.91427000-3.21687700$

$\begin{array}{lll}4.40177700 & -1.59230800 & -2.90800200\end{array}$

\begin{tabular}{rrr}
1.77540300 & -5.80368300 & -1.98970500 \\
\hline .57505400 & -4.8068300 & 1.21930600
\end{tabular}

$1.57905400-4.80688700 \quad 0.70544200$

$\begin{array}{lll}1.35199500 & -6.23816200 & 1.61688400\end{array}$

$\begin{array}{lll}0.49779400 & -4.84199400 & 2.00697300\end{array}$

$\begin{array}{lll}-3.12966800 & -4.31704400 & 1.58415300\end{array}$

$\begin{array}{lll}-4.20221000 & -4.40224700 & 1.84771300\end{array}$

$\begin{array}{lll}-2.29082100 & -4.51765700 & 2.85797900\end{array}$

$\begin{array}{lll}-2.56081900 & -3.79009800 & 3.64853400\end{array}$

$\begin{array}{lll}-1.20778200 & -4.35795000 & 2.65848600\end{array}$

$\begin{array}{lll}-4.87158400 & -1.76648600 & 2.07266000\end{array}$

$\begin{array}{lll}-5.19850600 & -2.79775500 & 2.31046700\end{array}$

$\begin{array}{lll}-5.75321500 & -1.27958900 & 1.61195100\end{array}$

$\begin{array}{lll}-4.52685600 & -1.02431300 & 3.37693700\end{array}$

$\begin{array}{lll}-4.22906900 & 0.02541500 & 3.18359900\end{array}$

$\begin{array}{lll}-3.68785800 & -1.51531700 & 3.90940500\end{array}$

$\begin{array}{lll}-5.40177400 & -1.00978300 & 4.05650200\end{array}$

$\begin{array}{lll}-4.90288400 & 0.89592500 & 0.43770900\end{array}$

$\begin{array}{llll}-5.94510900 & 0.22471000 & -0.23552900\end{array}$

$\begin{array}{lll}-7.29572500 & 0.53102300 & -0.05129600\end{array}$

$\begin{array}{llll}-8.08163000 & -0.00801700 & -0.60317300\end{array}$

$\begin{array}{llll}-7.63865000 & 1.54371800 & 0.86048500\end{array}$

$\begin{array}{llll}-8.69743100 & 1.79428100 & 1.0198500\end{array}$

$\begin{array}{llll}-6.697411700 & 2.72350100 & 1.01929800\end{array}$

$-6.63091700-3.01240800 \quad 2.28054200$

$\begin{array}{lll}-6.89691700 & 3.01240800 & 2.28054200\end{array}$

$-4.49008800-2.44522300 \quad 1.89095400$

$-4.49008800 \quad 2.44522300-1.89095400$

$-5.57848800-0.85550700-1.18232700$

$-6.41504500-1.34442700-1.54881200$

$-5.05176100-0.46761500-1.99557800$

$\begin{array}{lll}-4.59910100 & 2.14316100 & -2.91496600\end{array}$

$\begin{array}{lll}-5.62569200 & 2.21864200 & -3.32484600\end{array}$

$\begin{array}{lll}-4.39404100 & 1.07716700 & -2.66131300\end{array}$

$\begin{array}{lll}-3.88725100 & 2.38692200 & -3.72845500\end{array}$

$\begin{array}{lll}-4.40472500 & 3.05788600 & -1.69305400\end{array}$

$\begin{array}{llll}-4.47738700 & 4.11312600 & -2.02502200\end{array}$

$\begin{array}{lll}-5.24225400 & 2.92108400 & -0.98397300\end{array}$

$\begin{array}{lll}-2.98783800 & -0.71227100 & 0.52561700\end{array}$

$\begin{array}{lll}5.58646000 & -1.29084300 & 1.10868800\end{array}$

$\begin{array}{lll}6.97448100 & -1.45354000 & 1.09163000\end{array}$

$\begin{array}{lll}7.50062200 & -1.96403900 & 1.91306500\end{array}$

$\begin{array}{lll}7.69229400 & -0.94742500 & -0.00443400\end{array}$

$\begin{array}{lll}8.78412300 & -1.07187000 & -0.04198300 \\ 7.01408500 & -0.27962200 & -1.03769700\end{array}$

$\begin{array}{llll}7.57780400 & 0.12557300 & -1.89083700\end{array}$

$\begin{array}{lll}7.68661400 & -0.98235200 & 2.97087500\end{array}$

$\begin{array}{lll}-0.86056700 & -4.77272500 & -0.42258800\end{array}$

$\begin{array}{lll}-0.820366700 & -5.80022600 & 0.28524400\end{array}$

$-0.20366700-5.80022600-0.28524400$

$\begin{array}{lll}-1.83570200 & -5.17656300 & -1.35916500\end{array}$

$-0.49794000-7.15567100-0.11954300$

$-2.35955100-4.40217700-1.93726300$

$-2.35955100-4.40217700-1.93726300$

$\begin{array}{lll}0.03167900 & -7.92736100 & 0.69982600\end{array}$

$\begin{array}{lll}-2.89794000 & -6.81656800 & -2.30388100\end{array}$

$-1.72131800-8.58557600-0.96123100$

$1.27357200-5.69133400-1.55426100$

$2.80174900-5.13715100-2.22468100$

$\begin{array}{lll}1.05027700 & -4.43186300 & -3.29805100\end{array}$

$1.55612200-3.58950300-3.81065300$

$1.03425300-5.29654500-3.99086600$

$\begin{array}{llll}0.00122500 & -4.12613500 & -3.11585100\end{array}$

$\begin{array}{llll}-2.93861100 & -5.14131200 & 0.87432100\end{array}$

$\begin{array}{lll}5.18031000 & -2.87857300 & -0.78183400\end{array}$

$\begin{array}{llll}0.86182300 & 7.72002800 & -0.17058000\end{array}$

$\begin{array}{lll}-0.68197400 & 7.73091300 & 1.35929700\end{array}$

$\begin{array}{llll}2.38864000 & 7.39676400 & -1.69120400\end{array}$

$\begin{array}{llll}0.97224800 & 8.81383800 & -0.19162200\end{array}$

$\begin{array}{rrr}-1.03428500 & -0.56753700 & -0.28347300 \\ 0.86882500 & 0.64574100 & -0.15298400\end{array}$

$\begin{array}{lll}-2.42339700 & -5.53723600 & 3.26970400\end{array}$

\section{$\left[\alpha_{2} \beta_{2}-P\right]^{6+}$}

$\begin{array}{lrrr}C & -0.89135700 & 2.93026300 & -0.43953800 \\ \mathrm{C} & 3.94642500 & -1.00485800 & 1.12461100 \\ \mathrm{C} & 4.07307000 & 0.39874100 & 1.11185000 \\ \mathrm{C} & 2.93622800 & 0.93119500 & 0.37892400 \\ \mathrm{C} & 2.73063200 & -1.34966700 & 0.41537400 \\ \mathrm{C} & 2.24876700 & -2.64661100 & 0.05104400 \\ \mathrm{C} & -3.94365600 & 1.09281800 & 1.11061900 \\ \mathrm{C} & -4.06908600 & -0.31077500 & 1.11353500\end{array}$ $\begin{array}{lll}-2.93122600 & -0.86252300 & 0.40552200\end{array}$

$\begin{array}{lll}-2.67844600 & -2.22445700 & 0.04753600\end{array}$

$\begin{array}{lll}-1.39622300 & -2.75316100 & -0.27486200 \\ -1.05451700 & -3.98267600 & -0.97520200\end{array}$

$\begin{array}{llll}-1.05451700 & -3.98267600 & -0.97520200\end{array}$

$\begin{array}{lll}0.35151800 & -4.10072600 & -0.97731200\end{array}$

$\begin{array}{llll}0.89586900 & -2.94764500 & -0.27499400\end{array}$

$\begin{array}{lll}1.04696900 & 3.93071500 & -1.18188200\end{array}$

$\begin{array}{lll}1.38264700 & 2.73449000 & -0.43845600\end{array}$

$\begin{array}{llll}2.66419900 & 2.26772800 & -0.02126400\end{array}$

$\begin{array}{lll}-2.23382700 & 2.68967700 & -0.02228400\end{array}$

$\begin{array}{llll}0.18262200 & 2.10383600 & -0.09439300\end{array}$

$\begin{array}{llll}0.12910200 & 1.49608600 & 0.72968500\end{array}$

$\begin{array}{llll}-0.35568100 & 4.05167800 & -1.18227300\end{array}$

$\begin{array}{llll}1.99559400 & 4.79692500 & -1.96014800\end{array}$

$\begin{array}{llll}2.84276300 & 5.10866000 & -1.32196500\end{array}$

$\begin{array}{llll}1.48197900 & 5.73685200 & -2.23957900\end{array}$

$\begin{array}{lll}-2.10855800 & 0.20742800 & 0.05033900\end{array}$

$\begin{array}{lll}-1.44214600 & 0.13767600 & -0.72418700\end{array}$

$\begin{array}{lll}-0.18945000 & -2.13429000 & 0.07009800\end{array}$

$\begin{array}{lll}-0.13002600 & -1.41430100 & 0.79444900\end{array}$

$\begin{array}{lll}2.10816500 & -0.15377100 & 0.05786000\end{array}$

$1.43817700-0.10987500-0.71525700$

$\begin{array}{llll}2.51086000 & 4.11373600 & -3.24035100\end{array}$

$\begin{array}{llll}1.70720700 & 3.96555400 & -3.98767900\end{array}$

$2.90081000 \quad 3.09044300-3.03275900$

$\begin{array}{llll}3.30208000 & 4.71961400 & -3.72268200\end{array}$

$\begin{array}{llll}3.72889600 & 3.30928000 & 0.12329600\end{array}$

$\begin{array}{llll}4.92253600 & 3.36255100 & -0.63352900\end{array}$

$\begin{array}{llll}5.89118600 & 4.35032100 & -0.43868100\end{array}$

$\begin{array}{llll}4.51291700 & 5.30609500 & 1.31983300\end{array}$

$\begin{array}{lll}3.54455500 & 4.31509600 & 1.09863800\end{array}$

$\begin{array}{llll}2.62570000 & 4.30659500 & 1.70225000\end{array}$

$\begin{array}{llll}5.15291400 & 2.37955000 & -1.72129300\end{array}$

$\begin{array}{llll}4.52327100 & 2.57003200 & -2.53427300\end{array}$

$\begin{array}{llll}4.98515800 & 1.40063000 & -1.41679800\end{array}$

$\begin{array}{llll}6.12916900 & 2.43075100 & -2.07290900\end{array}$

$\begin{array}{llll}5.09097200 & 1.16738400 & 1.90847000\end{array}$

$\begin{array}{llll}5.49284500 & 2.02291200 & 1.33623400\end{array}$

$\begin{array}{lll}5.95811000 & 0.50727200 & 2.10399800\end{array}$

$\begin{array}{lll}4.52694100 & 1.67643600 & 3.25040500\end{array}$

$\begin{array}{lll}3.66453100 & 2.35605700 & 3.10504800\end{array}$

$\begin{array}{lll}5.30994700 & 2.23420800 & 3.79940600\end{array}$

$\begin{array}{llll}4.19477300 & 0.83996100 & 3.89543100\end{array}$

$\begin{array}{lll}4.81545700 & -1.94515300 & 1.91090900\end{array}$

$\begin{array}{lll}5.12003700 & -2.80373700 & 1.28433300\end{array}$

$\begin{array}{lll}5.75857800 & -1.43127400 & 2.17761500\end{array}$

$4.13992900-2.43438300 \quad 3.20591400$

$4.01627000-1.61916200 \quad 3.94513300$

$4.73985500-3.22796800 \quad 3.69250500$

$\begin{array}{lll}4.10463000 & -2.80348900 & 3.01926800\end{array}$

$3.31298100-3.68509800-0.14531600$

$\begin{array}{llll}4.25336500 & -3.47372800 & -1.17846700\end{array}$

$4.16978800-2.56293900-1.78727200$

$\begin{array}{lll}2.56497800 & -5.12393400 & 1.76498700\end{array}$

$\begin{array}{lll}1.56487600 & -5.04963600 & 1.49968100\end{array}$

$\begin{array}{lll}2.71328700 & -6.07531000 & 2.15570900\end{array}$

$\begin{array}{lll}1.11241600 & -5.12135200 & -1.77905500\end{array}$

$0.45591900-5.99538500-1.95320600$

$\begin{array}{llll} & 1.59123400 & -4.57292600 & -3.13902800\end{array}$

$\begin{array}{llll}0.74023900 & -4.25803900 & -3.77304000\end{array}$

$\begin{array}{llll}2.14552700 & -5.35989400 & -3.68584600\end{array}$

$2.26488600-3.70218000-3.02115700$

$-1.97801000-4.86548200-1.76906200$

$\begin{array}{lll}-3.44509600 & -4.57464400 & 1.80462300\end{array}$

$\begin{array}{lll}-2.44497900 & -4.70113000 & 1.56106700\end{array}$

$\begin{array}{lll}-3.77718500 & -5.46642500 & 2.22157500\end{array}$

$\begin{array}{lll}-3.50409500 & -3.84317400 & 2.55183100\end{array}$

$\begin{array}{lll}-5.09520900 & -1.08559600 & 1.89043300\end{array}$

$\begin{array}{lll}-5.94436100 & -0.42011000 & 2.13718100\end{array}$

$\begin{array}{lll}-4.53045800 & -1.66953800 & 3.19979000\end{array}$

$-4.30699100-0.88193700 \quad 3.94542400$

$\begin{array}{lll}-3.55457300 & -2.18447700 & 3.03805700\end{array}$

$-4.80050300 \quad 2.026889001 .91990800$

$\begin{array}{lll}-5.76270400 & 1.52518500 & 2.13922800\end{array}$

$\begin{array}{lll}-5.06323200 & 2.93492600 & 1.34730700\end{array}$

$\begin{array}{llll}-4.12935300 & 2.44169100 & 3.24510500\end{array}$

$\begin{array}{lll}-3.16092700 & 2.95151900 & 3.07477300\end{array}$

$-3.94176000 \quad 1.5678$

$\begin{array}{lll}-4.78838800 & 3.13913500 & 3.79740400\end{array}$

$\begin{array}{lll}-3.10205400 & 3.89919700 & 0.12557600\end{array}$

$\begin{array}{lll}-4.26944500 & 4.16021100 & -0.62905100\end{array}$

$\begin{array}{llll}-5.05107400 & 5.30097500 & -0.43081300\end{array}$

$\begin{array}{lll}-5.94875800 & 5.48496600 & -1.04179900\end{array}$

$\begin{array}{lll}-4.67886500 & 6.22442400 & 0.56237900\end{array}$

$\begin{array}{lll}-5.29496000 & 7.12042600 & 0.72672700\end{array}$

$\begin{array}{lll}-3.52387800 & 6.00015900 & 1.32601900\end{array}$

$\begin{array}{lll}-3.22398500 & 6.72107800 & 2.10053400\end{array}$

$\begin{array}{lll}-2.74345100 & 4.85594600 & 1.10163300\end{array}$

$\begin{array}{lll}-1.83924300 & 4.68452600 & 1.70313700\end{array}$

$\begin{array}{llll}-4.66750800 & 3.23337600 & -1.71752400\end{array}$

$-5.61612000 \quad 3.45940200-2.07651400$

$\begin{array}{lll}-4.68182300 & 2.44128600 & -1.07651400\end{array}$

$\begin{array}{lll}-4.68182300 & 2.24128600 & -1.40947000 \\ -4.00864900 & 3.30475400 & -2.52650700\end{array}$

$-1.76484600 \quad 4.48631700-3.24207000$

$-2.44604000 \quad 5.21741300-3.71868400$

$-2.31781300 \quad 3.53983200-3.04008100$

$\begin{array}{lll}-2.31781300 & 3.53983200 & -3.04008100\end{array}$

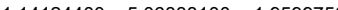

$-1.14124400 \quad 5.06838100-1.95997500$

$-0.47378800 \quad 5.90671500-2.23748600$

$\begin{array}{lll}-1.92292900 & 5.52012900 & -1.32200900\end{array}$

$\begin{array}{lll}-2.73263300 & 1.41966800 & 0.37622600\end{array}$

$\begin{array}{lll}3.46565300 & -4.86761000 & 0.61584000 \\ 4.47703600 & -5.79869900 & 0.36767900\end{array}$

$\begin{array}{lll}4.57500600 & -6.70437400 & 0.98668100\end{array}$

$\begin{array}{lll}5.38141100 & -5.568699400 & -0.68409600\end{array}$

$\begin{array}{lll}6.17508000 & -6.30214200 & -0.88775400 \\ 5.26822500 & -4.40265600 & -1.45445300\end{array}$

$\begin{array}{lll}5.26822500 & -4.40265600 & -1.45445300 \\ 5.97519200 & -4.21132600 & -2.27489000\end{array}$

$\begin{array}{rrr}5.97519200 & -4.21132600 & -2.27489000 \\ 2.74350800 & -4.44029100 & 2.53749900\end{array}$

$\begin{array}{lll}-3.90183300 & -3.07099600 & -0.14909700\end{array}$

$\begin{array}{lll}-4.26498600 & -4.19507400 & 0.62940000\end{array}$

$\begin{array}{lll}-4.77393000 & -2.72656800 & -1.20570100\end{array}$

$\begin{array}{lll}-5.41336900 & -4.94882800 & 0.37464600\end{array}$

$\begin{array}{lll}-5.92361700 & -3.47962400 & -1.48945000\end{array}$

$\begin{array}{lll}-4.52832300 & -1.85461400 & -1.82719300\end{array}$

$\begin{array}{lll}-6.24491700 & -4.59438400 & -0.70225400\end{array}$

$\begin{array}{lll}-5.67488600 & -5.81095100 & 1.00843300\end{array}$

$\begin{array}{lll}-6.57252200 & -3.18999200 & -2.32873300\end{array}$

$-7.14570900-5.18943900-0.91139500$

$\begin{array}{lll}-7.14570665700 & -5.189427700 & -1.21195100\end{array}$

$\begin{array}{lll}-2.9065700 & -5.08427700 & -1.21195100 \\ -1.48577500 & -5.84486500 & -1.92413500\end{array}$

$\begin{array}{lll}-1.48577500 & -5.84486500 & -1.92413500 \\ -2.34146500 & -4.26309700 & -3.14195200\end{array}$

$\begin{array}{lll}-1.34146500 & -4.26309700 & -3.14195200 \\ -1.44388600 & -4.12472500 & -3.77454700\end{array}$

$\begin{array}{lll}-1.44388600 & -4.12472500 & -3.77454700 \\ -3.03055700 & -4.94321700 & -3.67869100\end{array}$

$-3.03055700-4.94321700-3.67869100$

$\begin{array}{lll}-2.84090500 & -3.27964000 & -3.04509800\end{array}$

$1.5870000-1.51011800-1.21996300$

$1.98280900-5.51011800-1.21996300$

$\begin{array}{lll}5.68794700 & 5.32537800 & 0.55404000 \\ 4.34426500 & 6.06897800 & 2.09387200\end{array}$

$\begin{array}{lll}4.34426500 & 6.06897800 & 2.09387200\end{array}$

$\begin{array}{lll}6.80553400 & 4.37497400 & -1.05239500\end{array}$

$6.45088500 \quad 6.100536$

$\begin{array}{lll}-5.25174500 & -2.36623500 & 3.66975800\end{array}$

158

$\left.\alpha_{2} \beta_{2}-\mathrm{P}\left[\mathrm{H}_{2} \mathrm{SO}_{4} \cdot \mathrm{H}_{2} \mathrm{O}\right]_{2}\right]^{4+}$

$\begin{array}{lll}0.10217123 & 3.05725223 & 0.43049660\end{array}$ $\begin{array}{lll}3.94923820 & -1.93049786 & 0.85245221 \\ 4.34342219 & -0.60150560 & 1.06600970\end{array}$ $\begin{array}{lll}3.38181417 & 0.24681794 & 0.37627762\end{array}$ $\begin{array}{llll}2.74843298 & -1.89191501 & 0.03274140\end{array}$ $\begin{array}{lll}1.97109778 & -2.97058732 & -0.46630425\end{array}$ $\begin{array}{lll}-2.95143835 & 1.57964517 & 2.27397992\end{array}$ $\begin{array}{llll}-3.30778943 & 0.22297986 & 2.27927481\end{array}$ $\begin{array}{lll}-3.30778943 & 0.22297986 & 2.25927481 \\ -2.49309292 & -0.42225027 & 1.24058363\end{array}$ $\begin{array}{lll}-2.49309292 & -0.42225027 & 1.24058363 \\ -2.61605840 & -1.70539217 & 0.64575914\end{array}$ $\begin{array}{lll}-2.61605840 & -1.70539217 & 0.64575914 \\ -1.55777843 & -2.30177866 & -0.10106425\end{array}$ $\begin{array}{lll}-1.55777843 & -2.30177866 & -0.10106425 \\ -1.63251808 & -3.21515661 & -1.23237725\end{array}$ $\begin{array}{llll}-1.63251808 & -3.21515661 & -1.23237725 \\ -0.30839774 & -3.57002799 & -1.56438088\end{array}$ $\begin{array}{lll}-0.30839774 & -3.57002799 & -1.56438088 \\ 0.56169124 & -2.87003647 & -0.63767394\end{array}$ $\begin{array}{llll}0.56169124 & -2.87003647 & -0.63767394\end{array}$ $\begin{array}{llll}2.10587504 & 3.81244213 & -0.42436494\end{array}$ $\begin{array}{lll}2.26598893 & 2.47058794 & 0.11991832 \\ 3.42445170 & 1.66448767 & 0.22198912\end{array}$ $\begin{array}{lll}3.42445170 & 1.66448767 & 0.22198912\end{array}$ $\begin{array}{llll}-1.22100385 & 2.96284886 & 0.91923203\end{array}$ $\begin{array}{lll}1.03575142 & 2.04985825 & 0.59036071 \\ 0.91997167 & 1.21841014 & 1.21802732\end{array}$ $\begin{array}{llll}0.91997167 & 1.21841014 & 1.21802732\end{array}$ $\begin{array}{llll}0.76526166 & 4.16975597 & -0.25147119\end{array}$ $\begin{array}{llll}0.12296309 & 4.61935762 & -1.18456021\end{array}$ $\begin{array}{llll}4.07089342 & 4.67609413 & -0.61909420\end{array}$ $\begin{array}{llll}2.75835839 & 5.66318642 & -1.24918251\end{array}$ $\begin{array}{llll}3.24595102 & 0.67590896 & -3.00247423\end{array}$ $\begin{array}{llll}2.41258216 & 1.22523529 & -2.86902618\end{array}$ $\begin{array}{llll}3.38161649 & 0.69308816 & -3.97132114\end{array}$ $\begin{array}{lll}-1.59386725 & 0.51727521 & 0.76937016\end{array}$ $\begin{array}{lll}-0.99585284 & 0.36646538 & -0.07596615\end{array}$ $\begin{array}{lll}-0.22895368 & -2.10436642 & 0.18570475\end{array}$ $\begin{array}{lll}0.14825620 & -1.48173344 & 0.94850243\end{array}$ $2.43277218-0.56922855-0.19523221$ $\begin{array}{lll}2.43277218 & -0.56922855 & -0.1952322 \\ 1.63606140 & -0.25664395 & -0.79344535\end{array}$ $\begin{array}{llll}1.40694823 & 4.10478976 & -2.60554027\end{array}$ $\begin{array}{llll}3.40694823 & 4.10478976 & -2.60554027 \\ 2.49355658 & 4.11190350 & -3.23112861\end{array}$ $2.49355058 \quad 4.1100350-3.23112861$ $\begin{array}{llll}3.78570839 & 3.06432761 & -2.59964551 \\ 4.16766197 & 4.74227771 & -3.09719365\end{array}$ $\begin{array}{lll}4.16766197 & 4.74227771 & -3.09719365 \\ 4.76124424 & 2.30493042 & 0.20066189\end{array}$ $\begin{array}{llll}4.76724424 & 2.30493042 & 0.20066189\end{array}$ $\begin{array}{llll}5.76593649 & 1.88564842 & -0.70323723\end{array}$ $\begin{array}{lll}7.02979305 & 2.48363038 & -0.72906292\end{array}$ $\begin{array}{lll}7.79293305 & 2.14932616 & -1.45026201 \\ 7.32417908 & 3.51597284 & 0.17640038\end{array}$ $\begin{array}{llll}7.32417908 & 3.51597284 & 0.17648038\end{array}$ $\begin{array}{lll}8.31981462 & 3.98269859 & 0.16127163 \\ 6.35176148 & 3.94064828 & 1.09673799\end{array}$ $\begin{array}{lll}6.35176148 & 3.94064828 & 1.09673799\end{array}$ $\begin{array}{llll}6.58301816 & 4.74097648 & 1.81470329\end{array}$ $\begin{array}{lll}5.08307889 & 3.34651033 & 1.09978693\end{array}$ $\begin{array}{llll}4.31993164 & 3.67701814 & 1.81947963\end{array}$ $\begin{array}{llll}5.48776545 & 0.77528129 & -1.63169654\end{array}$ $\begin{array}{llll}4.52525203 & 0.84507999 & -2.18639705\end{array}$ $\begin{array}{llll}5.44429439 & -0.12608754 & -1.11278553\end{array}$ $\begin{array}{llll}6.24870222 & 0.69238306 & -2.32721918\end{array}$ $\begin{array}{lll}5.36027205 & -0.12544668 & 2.06900461\end{array}$ $\begin{array}{llll}6.07285392 & 0.59214280 & 1.61451903\end{array}$ $\begin{array}{lll}5.97435185 & -0.98985700 & 2.38988937\end{array}$ $\begin{array}{llll}0.90580998 & 1.99571472 & -2.76856958\end{array}$ $\begin{array}{llll}-0.58820573 & 0.21864782 & -3.79704688\end{array}$ $\begin{array}{llll}-0.83172114 & 0.81403816 & -4.54371275\end{array}$ $\begin{array}{llll}4.68897083 & 0.51746075 & 3.29719861\end{array}$ \begin{tabular}{llll}
4.13250939 & 1.43569966 & 3.29719861 \\
\hline
\end{tabular} $\begin{array}{llll}4.13250939 & 1.43569966 & 3.01948800 \\ 5.45036939 & 0.80036551 & 4.05032748\end{array}$ $\begin{array}{lll}5.45036939 & 0.80036551 & 4.05032748\end{array}$ \begin{tabular}{lll}
3.96318736 & -0.17292804 & 3.77063976 \\
\hline 4.47167744 & 3.14236864 & 1.57300927
\end{tabular} $4.74129137-3.142308640-1.57300927$ $\begin{array}{lll}4.74129137 & -3.94308478 & 0.85703988 \\ 5.41514114 & -2.87353518 & 2.08717080\end{array}$ $\begin{array}{lll}5.41514114 & -2.87353518 & 2.08717080 \\ -1.54069582 & 1.88621416 & -2.17101372\end{array}$ $\begin{array}{lll}-1.54069582 & 1.88621416 & -2.17101372 \\ 3.46848679 & -3.67905657 & 2.6098884\end{array}$ $3.46848679-3.67905657-2.60988884$ 
SUPPORTING INFORVATION

$\begin{array}{lrrrl}\mathrm{O} & 0.01306842 & 0.06551625 & -1.44272740 & \mathrm{C} \\ \mathrm{C} & 3.87029451 & -4.21289823 & -1.52302585 & \mathrm{C} \\ \mathrm{H} & 4.23220227 & -3.24236672 & -1.89138948 & \mathrm{C} \\ \mathrm{N} & 0.91005982 & -5.62821941 & 0.35372848 & \mathrm{H} \\ \mathrm{H} & 0.71593376 & -4.98765254 & 1.19561051 & \mathrm{C} \\ \mathrm{H} & 0.13347921 & -5.39834876 & -0.30676630 & \mathrm{H} \\ \mathrm{C} & 0.16012661 & -4.24145854 & -2.82967942 & \mathrm{C} \\ \mathrm{H} & -0.69791020 & -4.74352231 & -3.31886385 & \mathrm{H} \\ \mathrm{C} & 0.79186430 & -3.22139773 & -3.79753829 & \mathrm{C} \\ \mathrm{H} & 0.08118581 & -2.40809337 & -4.04372197 & \mathrm{H} \\ \mathrm{H} & 1.09459774 & -3.71863790 & -4.73970998 & \mathrm{~N} \\ \mathrm{H} & 1.69155655 & -2.74736485 & -3.35417349 & \mathrm{H} \\ \mathrm{C} & -2.85076219 & -3.42932386 & -2.09397483 & \mathrm{H} \\ \mathrm{N} & -2.86902551 & -4.62893652 & 1.23493381 & \mathrm{H} \\ \mathrm{H} & -2.05582150 & -4.29334638 & 1.85488747 & \mathrm{C} \\ \mathrm{H} & -2.41741820 & -4.77512307 & 0.30557635 & \mathrm{C} \\ \mathrm{H} & -3.16108586 & -5.55473217 & 1.59548989 & \mathrm{H} \\ \mathrm{C} & -4.18625345 & -0.45527132 & 3.27537486 & \mathrm{H} \\ \mathrm{H} & -5.10468467 & 0.14880276 & 3.43113285 & \mathrm{H} \\ \mathrm{C} & -3.46452338 & -0.66937459 & 4.62090180 & \mathrm{H} \\ \mathrm{H} & -3.20092512 & 0.29230937 & 5.10178769 & \mathrm{H} \\ \mathrm{H} & -2.53206297 & -1.24920463 & 4.47842858 & \mathrm{O} \\ \mathrm{H} & -4.12380759 & -1.22141156 & 5.31955861 & \mathrm{H} \\ \mathrm{C} & -3.46259669 & 2.60913448 & 3.24346262 & \mathrm{C} \\ \mathrm{H} & -4.37535171 & 2.21237534 & 3.73081052 & \mathrm{C} \\ \mathrm{H} & -3.78858165 & 3.51848531 & 2.69959178 & \mathrm{C} \\ \mathrm{C} & -2.43640218 & 3.00368740 & 4.32073919 & \mathrm{H} \\ \mathrm{H} & -1.48118598 & 3.34426350 & 3.87610698 & \mathrm{C} \\ \mathrm{H} & -2.19970337 & 2.14679959 & 4.98002921 & \mathrm{H} \\ \mathrm{H} & -2.83450052 & 3.82116369 & 4.95322973 & \mathrm{C} \\ & & & & \end{array}$

$\begin{array}{llll}-2.05506677 & 4.19637747 & 0.96984684\end{array}$ $\begin{array}{llll}-3.15165491 & 4.33213760 & 0.09254471\end{array}$ $\begin{array}{llll}-3.96533596 & 5.46997261 & 0.08585461\end{array}$ $-4.80858862-5.55898784-0.61675982$ $\begin{array}{llll}-3.68969554 & 6.50746001 & 0.991527\end{array}$ $\begin{array}{lll}-4.32681797 & 7.40372105 & 0.99807872\end{array}$ $\begin{array}{lll}-2.60166972 & 6.40094380 & 1.87450786\end{array}$ $\begin{array}{lll}-2.38250488 & 7.21694471 & 2.57861040\end{array}$ $\begin{array}{lll}-1.78545244 & 5.26140725 & 1.85427361\end{array}$ $\begin{array}{lll}-0.92359791 & 5.18497235 & 2.53219584\end{array}$ $\begin{array}{llll}-3.44483285 & 3.23576975 & -0.85807406\end{array}$ $\begin{array}{llll}-4.02697547 & 3.57684870 & -1.64404613\end{array}$ $\begin{array}{llll}-3.97453449 & 2.47060311 & -0.39817463\end{array}$ $\begin{array}{llll}-2.57545993 & 2.47420927 & -1.29192359\end{array}$ $\begin{array}{llll}0.13905823 & 5.40164623 & -0.84495569\end{array}$ $-0.93505594 \quad 6.08824939-2.62100438$

$-1.25038343-4.36713659-2.20995692$

$0.28660332 \quad 4.81920526-2.96285607$ $0.2860332 \quad 4.81920526-2.90285607$ $-0.02218878-5.82772153-0.17030106$ $\begin{array}{lll}0.62218878 & 5.82772153 & -0.17030106\end{array}$ $\begin{array}{llll}-0.21189523 & 0.42679742 & 4.04374851\end{array}$ $\begin{array}{llll}-0.07929483 & 0.37341989 & 5.01782999\end{array}$ $\begin{array}{lll}-1.86506090 & 1.75180592 & 1.31974454\end{array}$ $2.20453064-5.50384851-0.34997415$ $2.91398491-6.68023609-0.60735668$ $\begin{array}{llll}2.53953896 & -7.65239659 & -0.24905315\end{array}$ $4.67302262-7.54610719-1.53711672$ $\begin{array}{llll}4.58636341 & -5.38376402 & -1.80195630\end{array}$ $\begin{array}{lll}5.51588630 & -5.33302999 & -2.38732088\end{array}$ $0.78370588-6.60371490 \quad 0.67614530$ $\begin{array}{lll}-3.92141348 & -2.40029210 & 0.72238596\end{array}$ $\begin{array}{llll}-4.05950649 & -3.76859417 & 1.0621916\end{array}$ $\begin{array}{lll}-5.11745078 & -1.69049683 & 0.4565191\end{array}$ $\begin{array}{lll}-5.30964762 & -4.38668636 & 1.17153680\end{array}$ $\begin{array}{lll}-6.37538395 & -2.29737879 & 0.54739328\end{array}$ $\begin{array}{lll}-5.03939255 & -0.63290127 & 0.16874273\end{array}$ $\begin{array}{lll}-6.47645792 & -3.64634994 & 0.92125459\end{array}$ $\begin{array}{lll}-5.38597362 & -5.45031765 & 1.44824840\end{array}$ $\begin{array}{lll}-7.28211425 & -1.71590762 & 0.32630052\end{array}$ $\begin{array}{lll}-7.45838513 & -4.13342288 & 1.00835174\end{array}$ $\begin{array}{lll}-3.71621769 & -3.80529000 & -1.50978579\end{array}$ $\begin{array}{llll}-2.62989587 & -4.21192541 & -2.84645677\end{array}$ $-3.25820773-2.12467052-2.80566838$ $\begin{array}{llll}-2.42395323 & -1.72059297 & -3.41208243\end{array}$ $\begin{array}{lll}-2.42395323 & -1.72059297 & -3.41208243 \\ -3.12466002 & -2.29999557 & -3.47272420\end{array}$ $-3.54211251-1.34066235-2.07442093$ $\begin{array}{lll}-3.54211251 & -1.34066235 & -2.07442093 \\ -4.53031723 & -1.43439939 & 2.89935893\end{array}$ $\begin{array}{lll}-4.53031723 & -1.43439939 & 2.89935893 \\ 0.55433729 & -0.87053973 & 3.37431097\end{array}$ $\begin{array}{lll}0.55433729 & -0.87053973 & 3.37431097 \\ 1.71926909 & -1.20852454 & 4.20740659\end{array}$ $\begin{array}{lll}1.71926909 & -1.20852454 & 4.20740659 \\ -0.50723048 & -1.95145771 & 3.26532936\end{array}$ $\begin{array}{lll}-0.50723048 & -1.95145771 & 3.26532936 \\ 0.85768858 & -0.34994342 & 1.97985224\end{array}$ $\begin{array}{lll}0.85768858 & -0.34994342 & 1.97985224\end{array}$ $\begin{array}{lll}-0.39088717 & -4.31962910 & 2.40123476\end{array}$ $\begin{array}{ccc}-0.29516664 & -4.81708706 & 3.24215141 \\ -0.31183851 & -3.32973591 & 2.72463185\end{array}$ $\begin{array}{llll}-0.31183851 & -3.32973591 & 2.72463185\end{array}$ $\begin{array}{lll}0.90021050 & -5.04205168 & -2.61951618\end{array}$ $\begin{array}{lll}-0.27906186 & 1.13535521 & -2.46787809\end{array}$ 


\section{SUPPORTINGINEORMATION}

\section{Experimental NMR Data}

$\alpha_{2} \beta_{2}-\mathbf{P} \cdot 10 \mathrm{CSA}(\mathbf{S})$

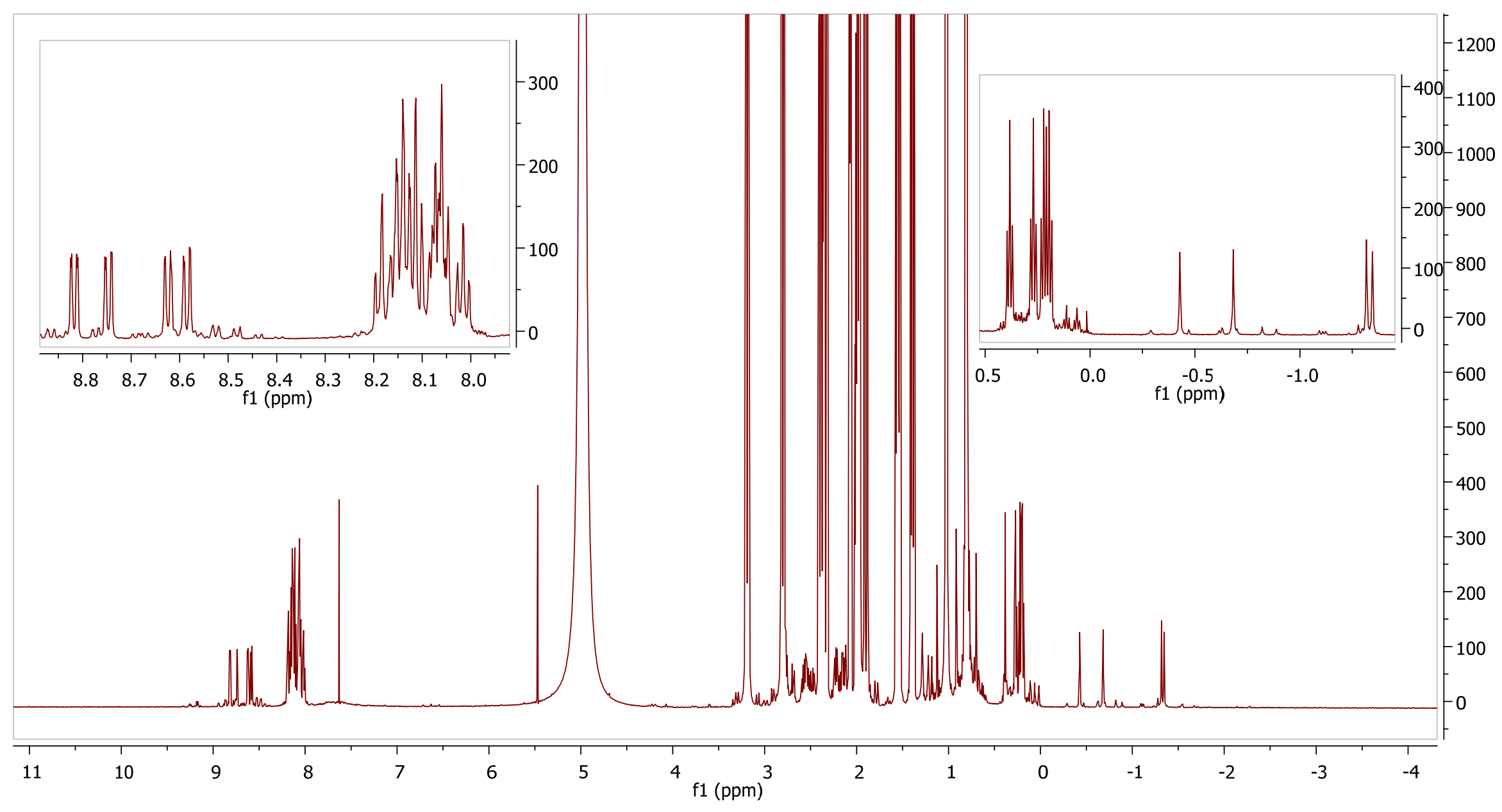

Figure S16. ${ }^{1} \mathrm{H}$ NMR spectrum of $\alpha_{2}, \beta_{2}-\mathbf{P} \cdot 10 \mathrm{CSA}(\mathbf{S})$ with the expansion of areas of interest $\left(600 \mathrm{MHz}\right.$, acetonitrile- $d_{3}, 20$ eq. of $\left.10 \mathrm{CSA}(\mathrm{S}), 25^{\circ} \mathrm{C}\right)$. 


\section{SUPPORTING INFORIVATION}

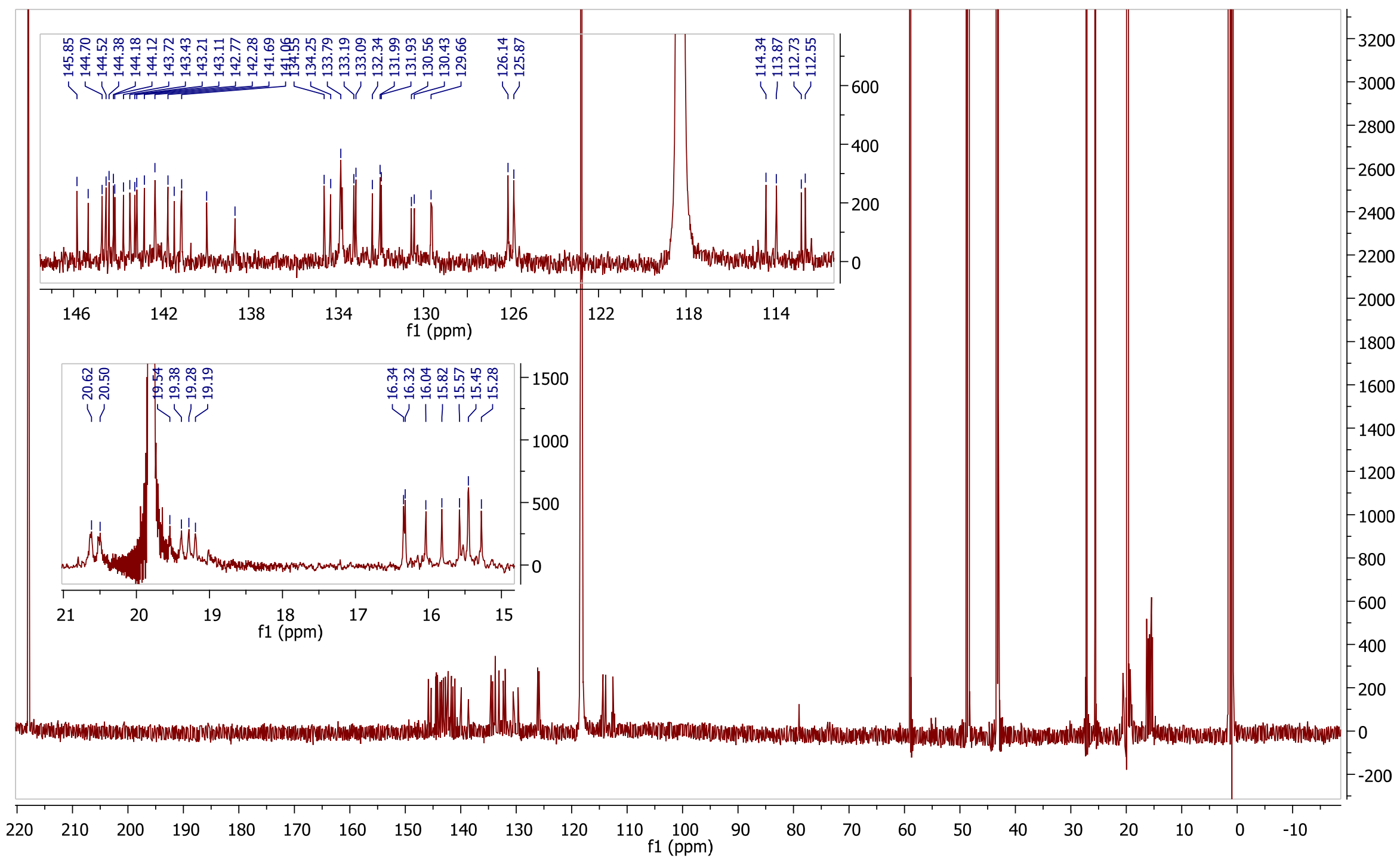

Figure S17. ${ }^{13} \mathrm{C}$ NMR spectrum of $\alpha_{2}, \beta_{2}-\mathrm{P} \cdot 10 \mathrm{CSA}(\mathbf{S})$ with expansion of areas of interest $\left(151 \mathrm{MHz}\right.$, acetonitrile- $d_{3}, 20$ eq. of $\left.10 \mathrm{CSA}(\mathrm{S}), 25^{\circ} \mathrm{C}\right)$. 


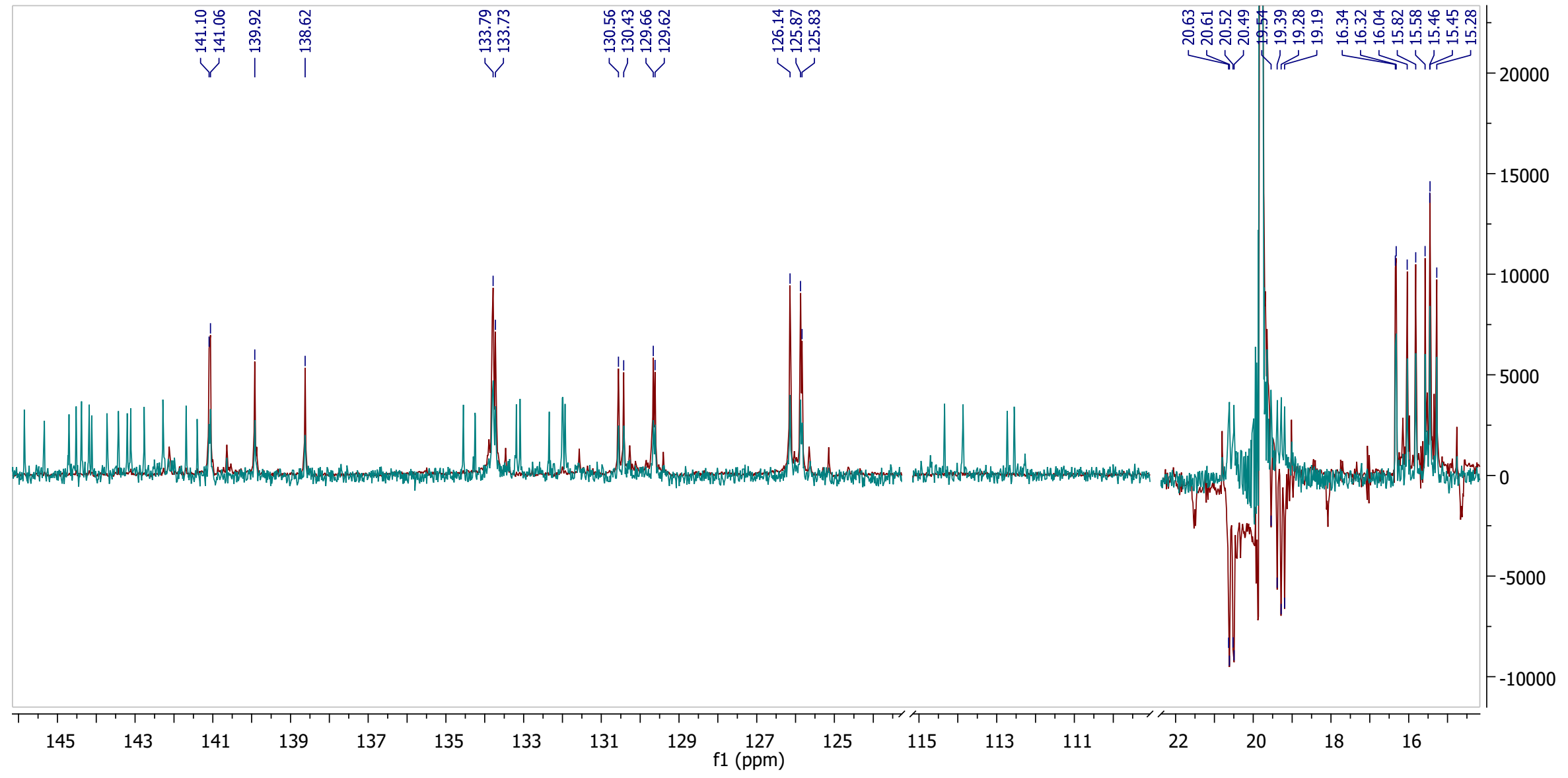

Figure S18. ${ }^{13} \mathrm{C}$ NMR (magenta) and DEPT-135 (red) overlay spectra of $\alpha_{2}, \beta_{2}-\mathrm{P} \cdot 10 \mathrm{CSA}(\mathbf{S})\left(151 \mathrm{MHz}\right.$, acetonitrile- $d_{3}, 20$ eq. of $\left.10 \mathrm{CSA}(\mathrm{S}), 25^{\circ} \mathrm{C}\right)$. 


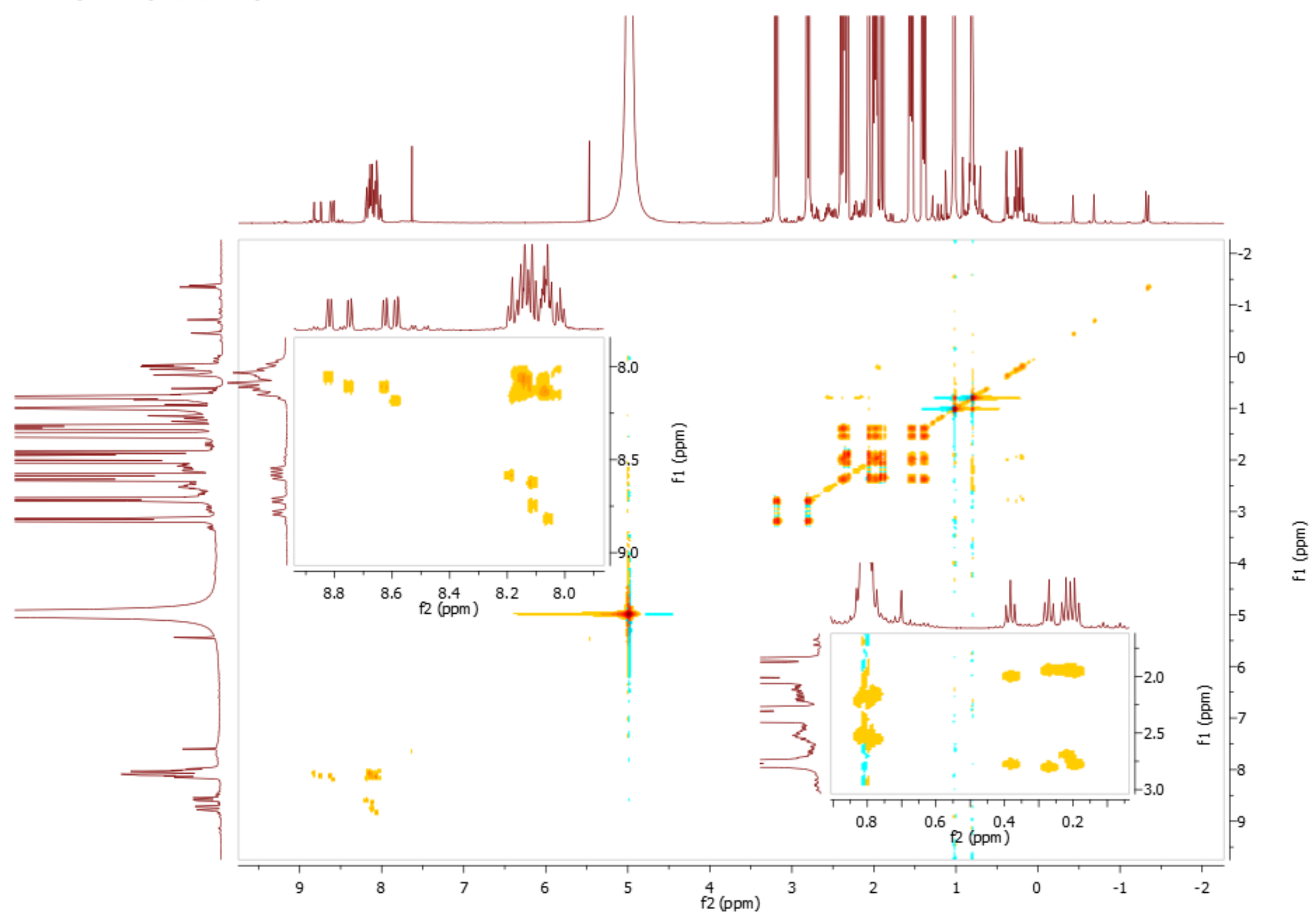

Figure S19. ${ }^{1} \mathrm{H}-{ }^{1} \mathrm{H}$ TOCSY spectrum of $\alpha_{2}, \beta_{2}-\mathrm{P} \cdot 10 \mathrm{CSA}(\mathbf{S})$ with expansion of areas of interest (acetonitrile- $d_{3}, 20$ eq. of $\left.10 \mathrm{CSA}(\mathrm{S}), 25^{\circ} \mathrm{C}\right)$. 


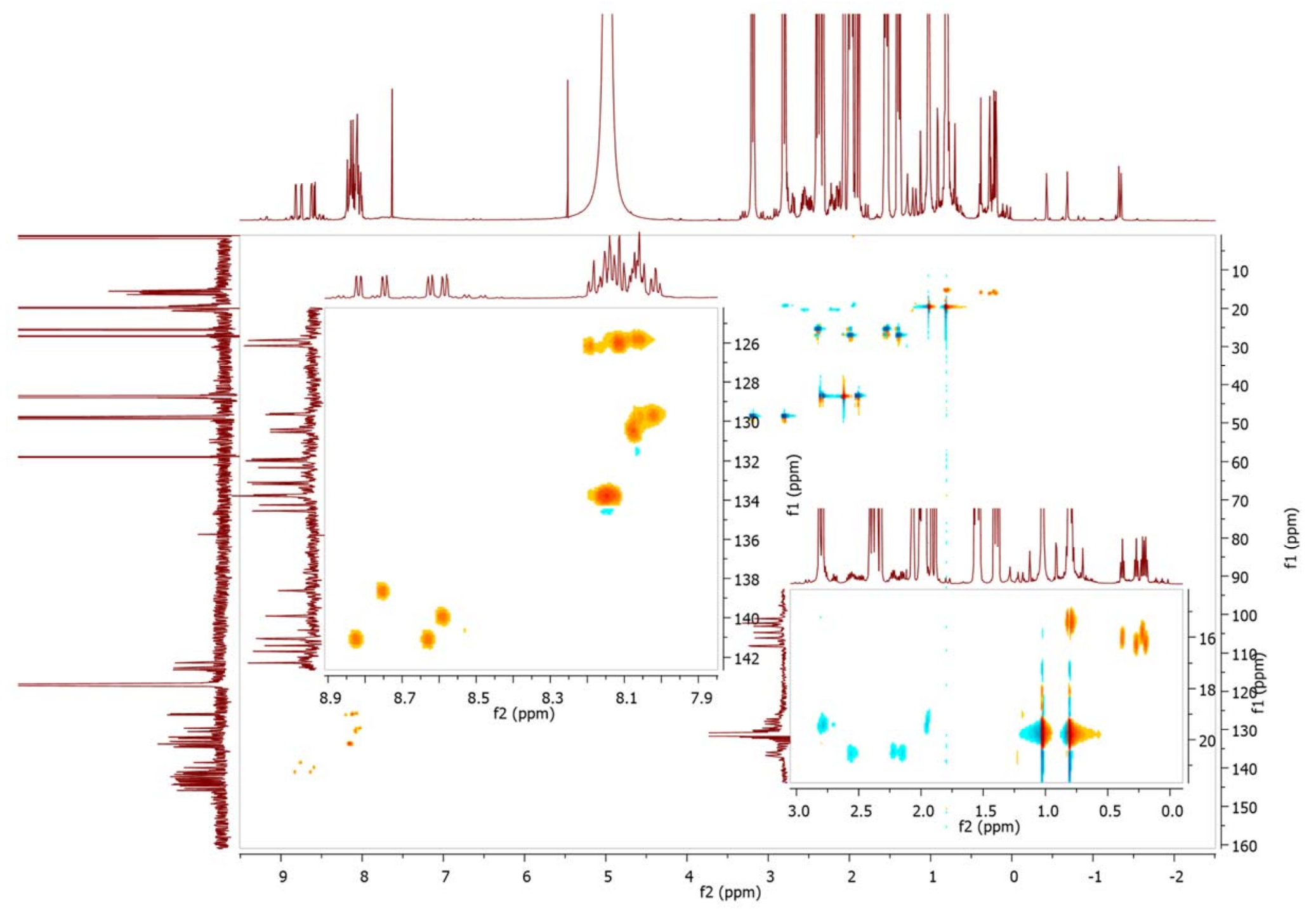

Figure S20. ${ }^{1} \mathrm{H}-{ }^{13} \mathrm{C}$ HSQC spectrum of $\alpha_{2}, \beta_{2}-\mathrm{P} \cdot 10 \mathrm{CSA}(\mathrm{S})$ with expansion of areas of interest (acetonitrile- $d_{3}, 20$ eq. of $\left.10 \mathrm{CSA}(\mathrm{S}), 25^{\circ} \mathrm{C}\right)$. 


\section{SUPPORTING INFORIMATION}

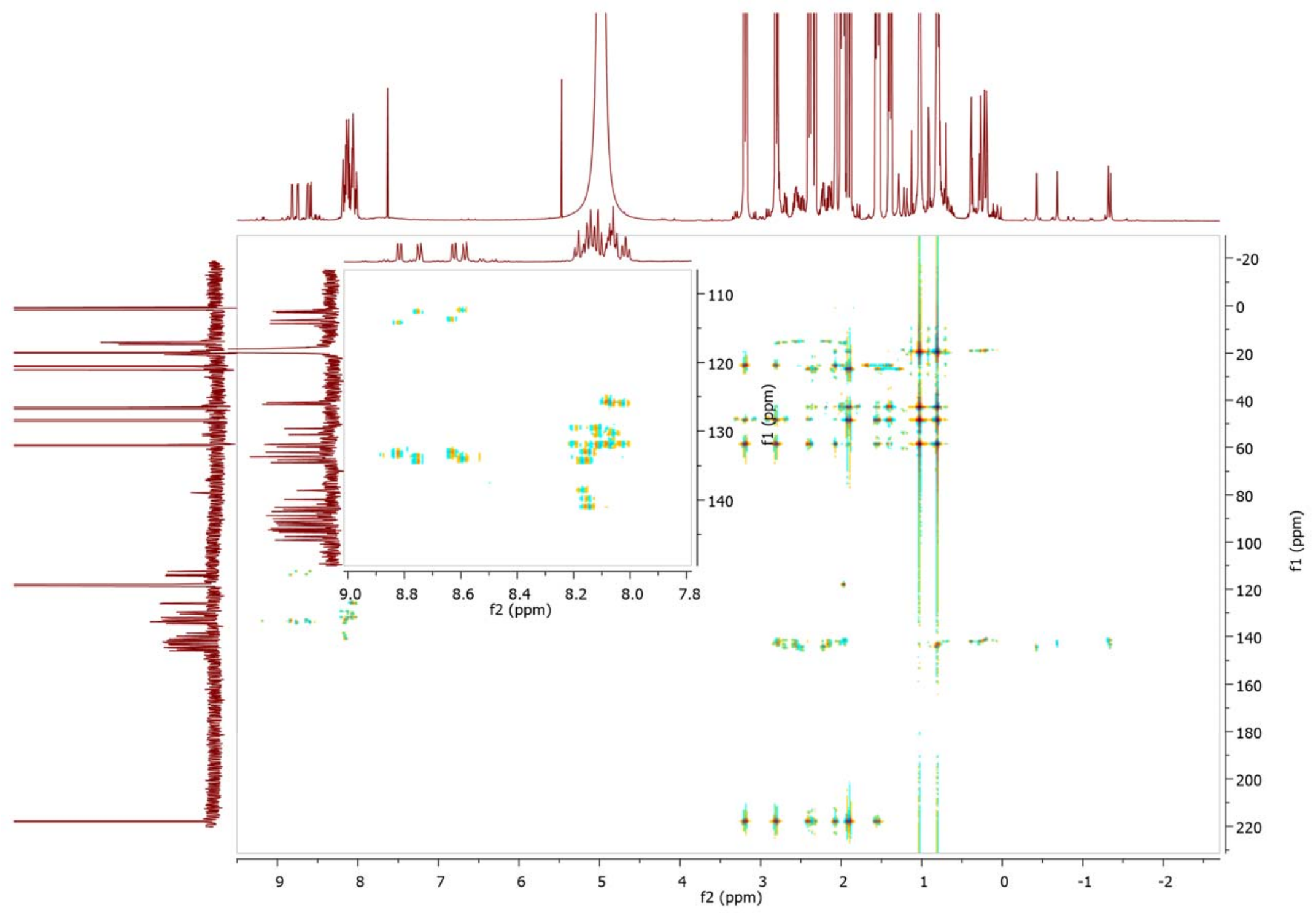




\section{SUPPORTING INFORVATION}

Figure S21. ${ }^{1} \mathrm{H}-{ }^{13} \mathrm{C}$ HMBC spectrum of $\alpha_{2}, \beta_{2}-\mathrm{P} \cdot 10 \mathrm{CSA}(\mathbf{S})$ with expansion of areas of interest (acetonitrile- $d_{3}, 20$ eq. of $\left.10 \mathrm{CSA}(\mathrm{S}), 25^{\circ} \mathrm{C}\right)$.

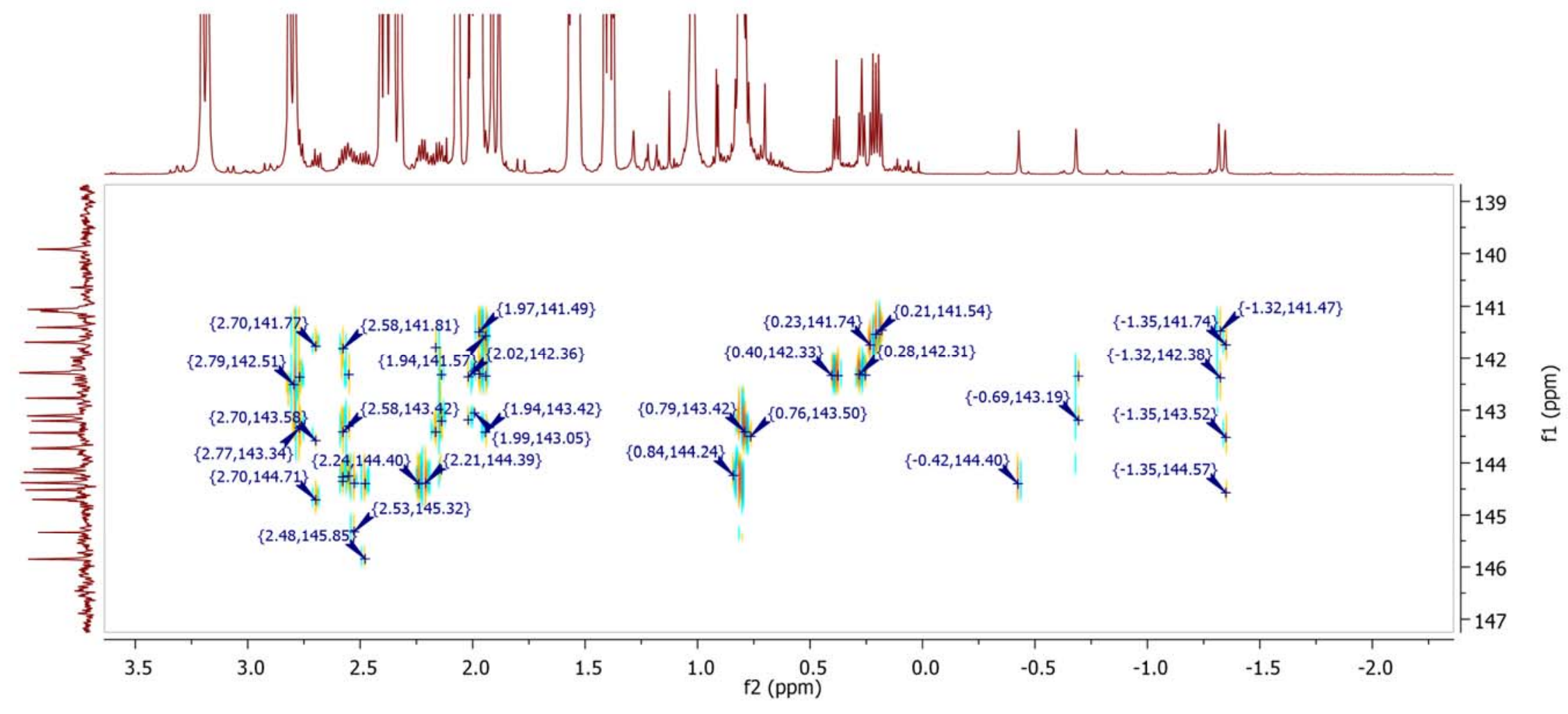

Figure S22. ${ }^{1} \mathrm{H}-{ }^{13} \mathrm{C}$ HMBC spectrum, expansion of area of interest of $\alpha_{2}, \beta_{2}-\mathrm{P} \cdot 10 \mathrm{CSA}(\mathrm{S})$ (acetonitrile- $d_{3}, 20$ eq. of $\left.10 \mathrm{CSA}(\mathrm{S}), 25^{\circ} \mathrm{C}\right)$. 
SUPPORTING INFORMATION

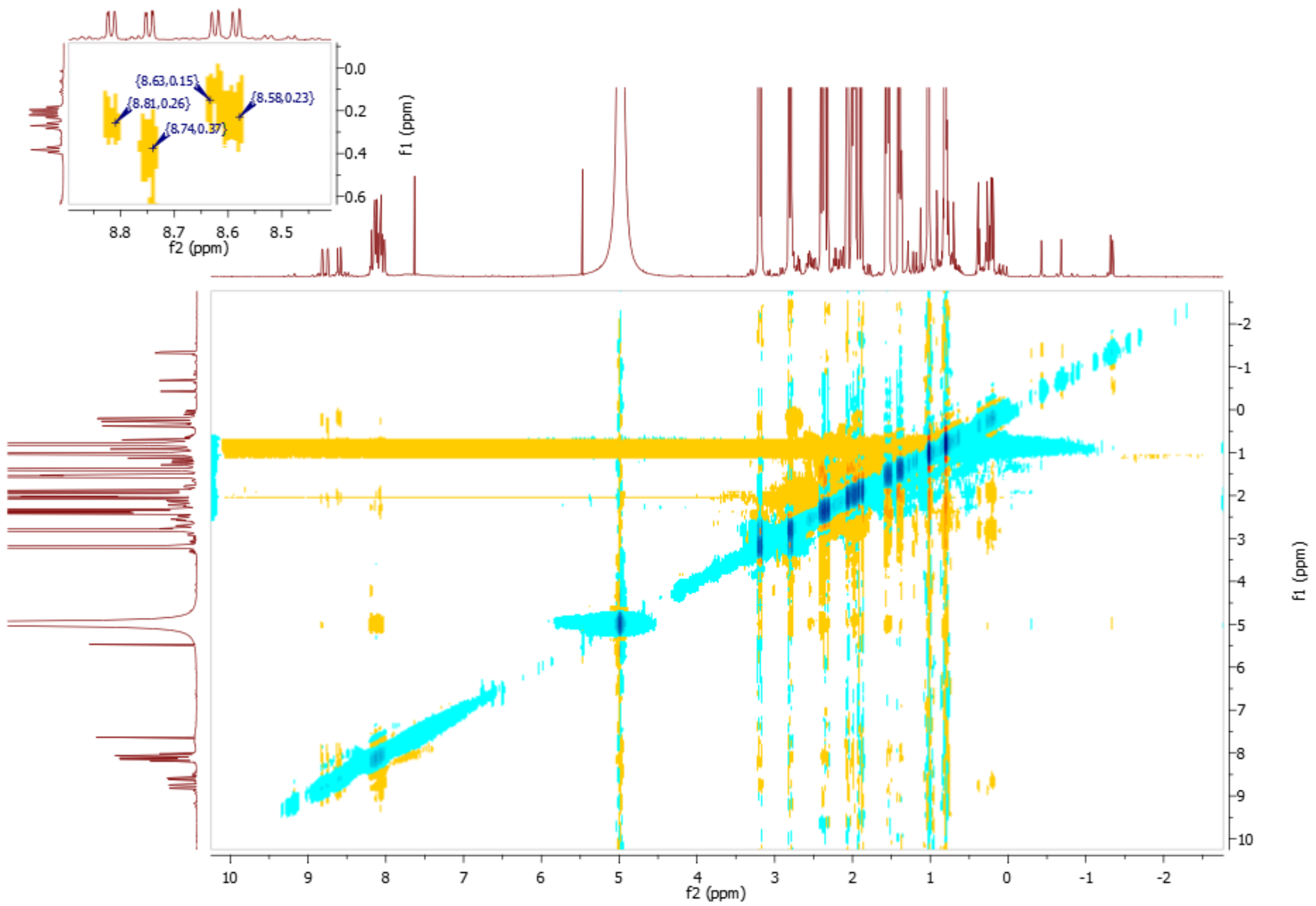

Figure S23. ${ }^{1} \mathrm{H}-{ }^{1} \mathrm{H}$ ROESY spectrum of $\alpha_{2}, \beta_{2}-\mathrm{P} \cdot 10 \mathrm{CSA}(\mathbf{S})$ with expansion of areas of interest (acetonitrile- $d_{3}, 20$ eq. of $\left.10 \mathrm{CSA}(\mathrm{S}), 25^{\circ} \mathrm{C}\right)$. 


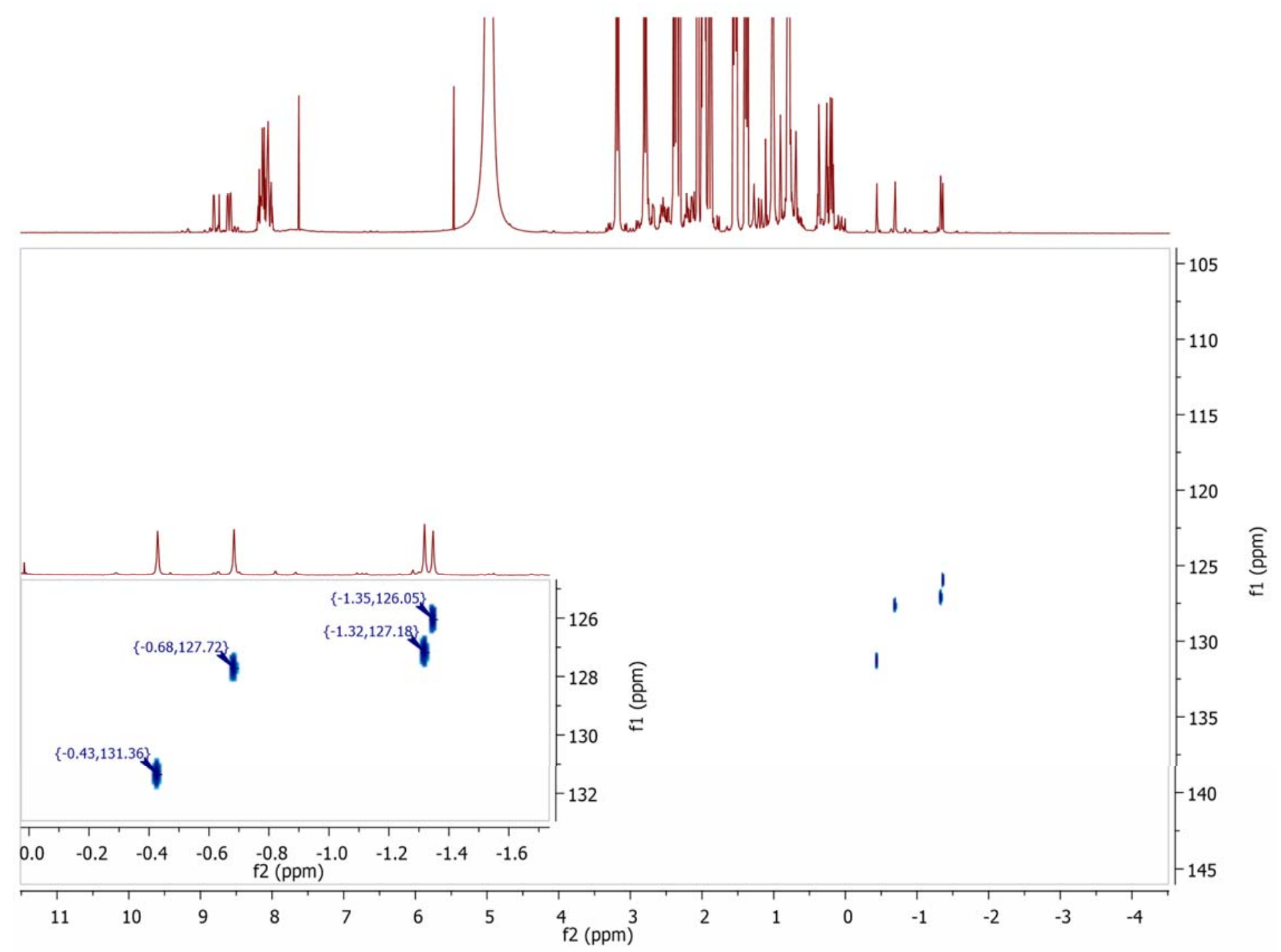

Figure S24. ${ }^{1} \mathrm{H}-{ }^{15} \mathrm{~N}$ HSQC spectrum of $\alpha_{2}, \beta_{2}-\mathrm{P} \cdot 10 \mathrm{CSA}(\mathbf{S})$ with expansion of areas of interest (acetonitrile- $d_{3}, 20$ eq. of $\left.10 \mathrm{CSA}(\mathrm{S}), 25^{\circ} \mathrm{C}\right)$. 


\section{SUPPORTING INFORVATION}

\section{$\alpha_{2} \beta_{2}-\mathrm{P}-10 \mathrm{CSA}(\mathrm{SR})$}

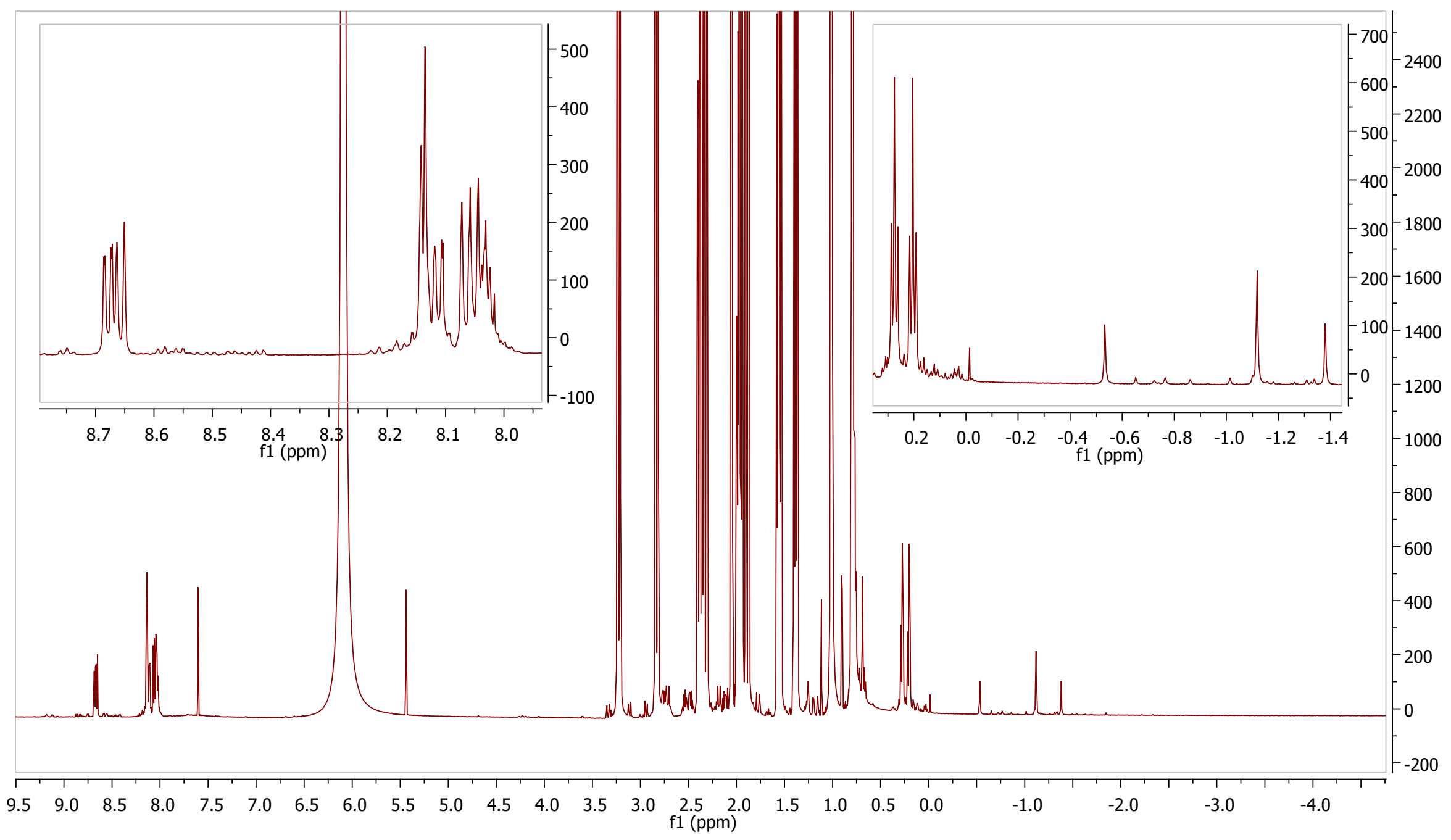




\section{SUPPORTING INFORMATION}

Figure S25. ${ }^{1} \mathrm{H}$ NMR spectrum of $\alpha_{2}, \beta_{2}-\mathrm{P} \cdot 10 \mathrm{CSA}(\mathrm{SR})$ with expansion of areas of interest (600 MHz, acetonitrile- $d_{3}, 20$ eq. of $10 \mathrm{CSA}(\mathrm{S})$ and $\left.10 \mathrm{CSA}(\mathrm{R}), 25^{\circ} \mathrm{C}\right)$.

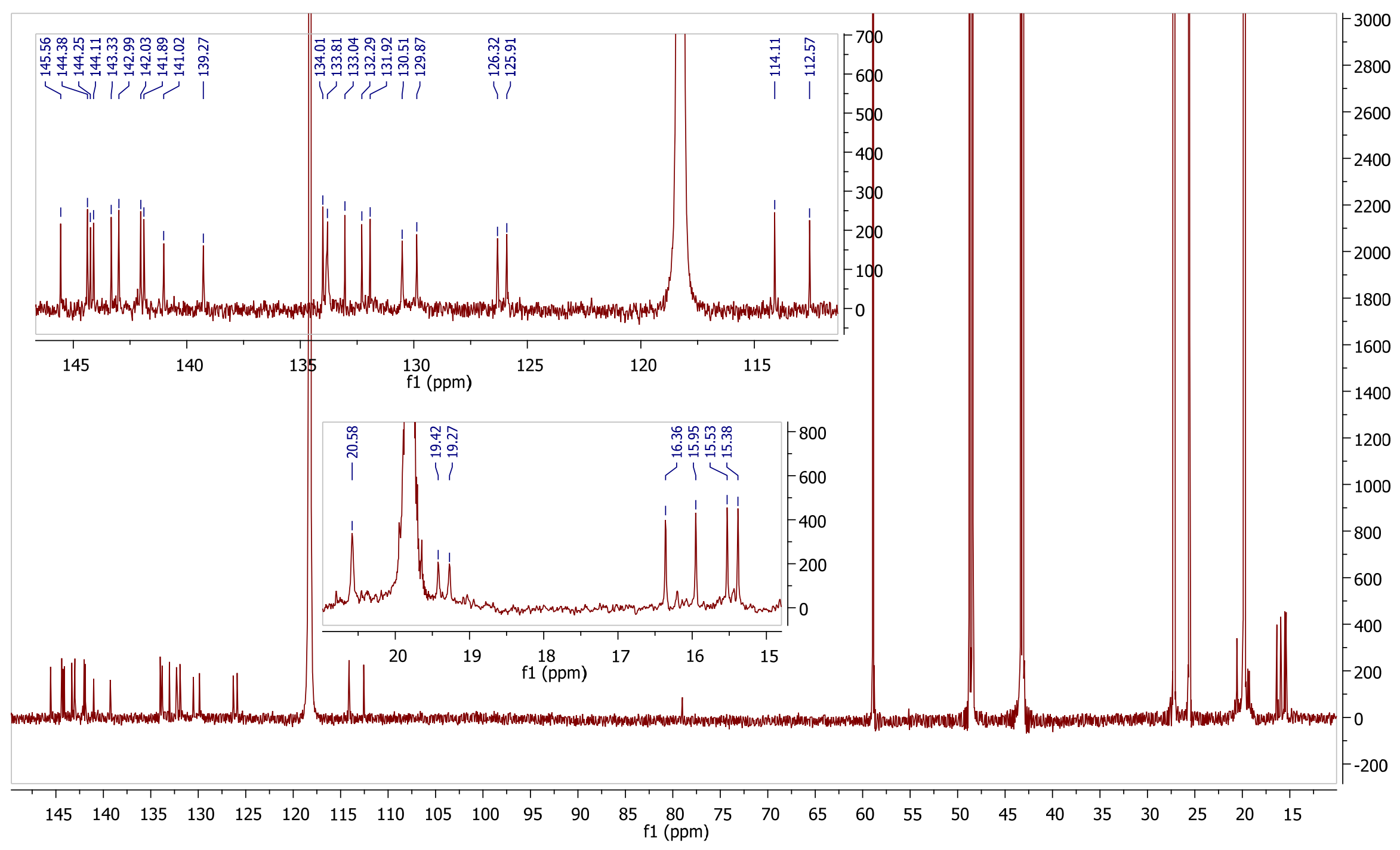

Figure S26. ${ }^{13} \mathrm{C}$ NMR spectrum of $\alpha_{2}, \beta_{2}-\mathbf{P} \cdot 10 \mathrm{CSA}(\mathrm{SR})$ with expansion of areas of interest (151 MHz, acetonitrile- $d_{3}, 20$ eq. of $10 \mathrm{CSA}(\mathrm{S})$ and $\left.10 \mathrm{CSA}(\mathrm{R}), 25^{\circ} \mathrm{C}\right)$. 


\section{SUPPORTING INFORMATION}

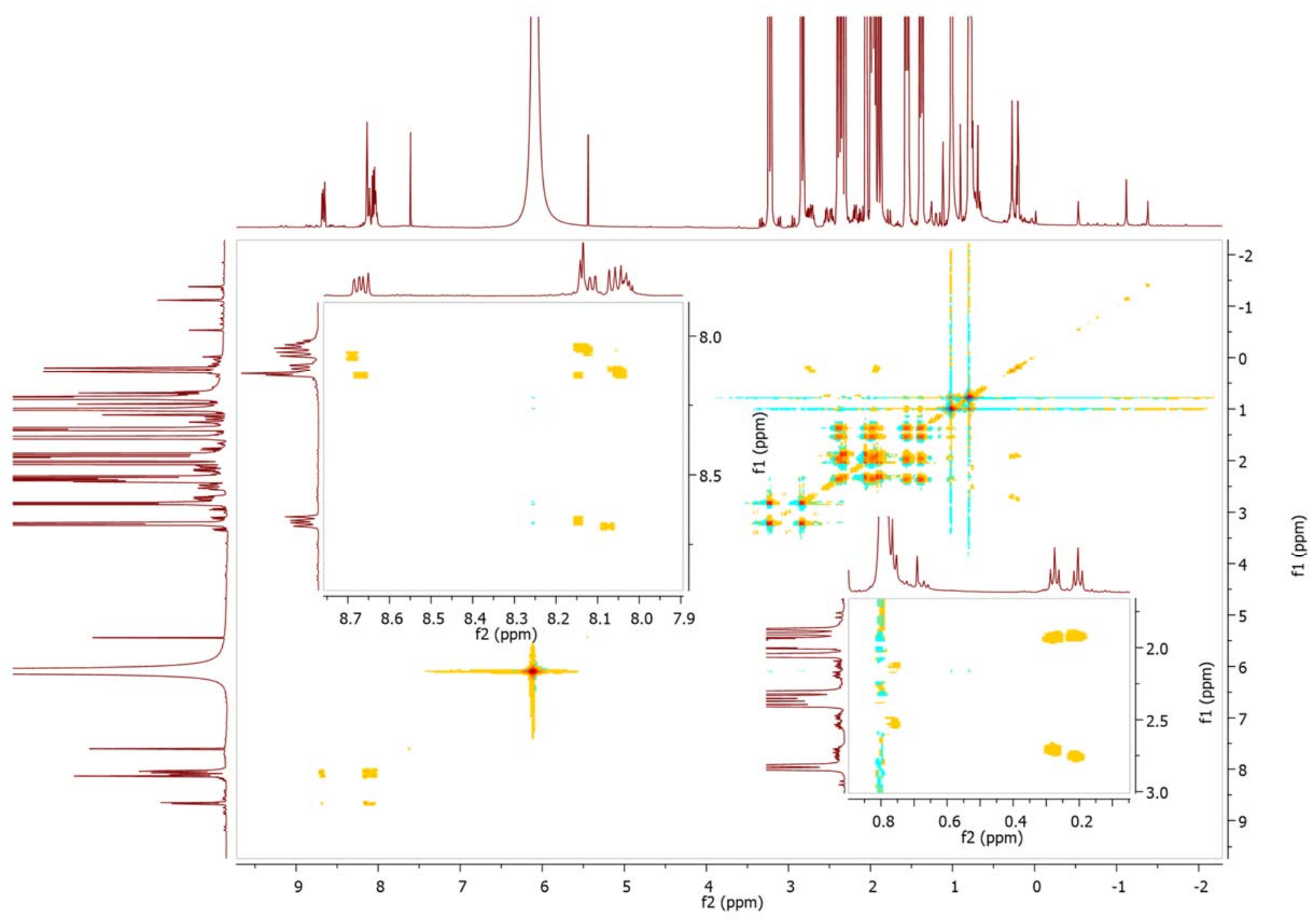


Figure S27. ${ }^{1} \mathrm{H}-{ }^{1} \mathrm{H}$ TOCSY spectrum of $\alpha_{2,} \beta_{2}-\mathrm{P} \cdot 10 \mathrm{CSA}(\mathrm{SR})$ with expansion of areas of interest (acetonitrile- $d_{3}, 20$ eq. of $10 \mathrm{CSA}(\mathrm{S})$ and $\left.10 \mathrm{CSA}(\mathrm{R}), 25^{\circ} \mathrm{C}\right)$.

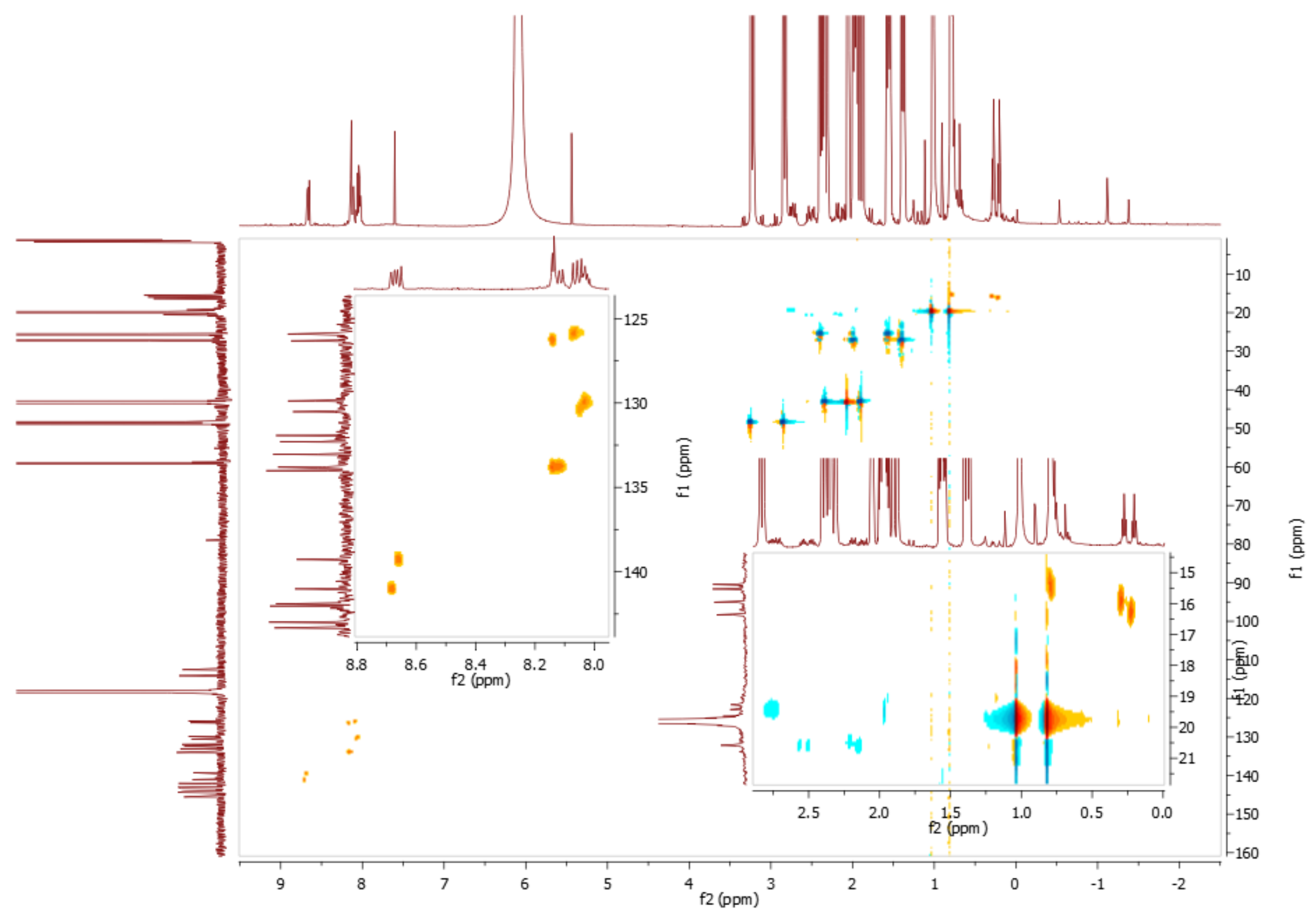




\section{SUPPORTING INFORVATION}

Figure S28. ${ }^{1} \mathrm{H}-{ }^{13} \mathrm{C}$ HSQC spectrum of $\alpha_{2}, \beta_{2}-\mathbf{P} \cdot 10 \mathrm{CSA}(\mathrm{SR})$ with expansion of areas of interest (acetonitrile- $d_{3}, 20$ eq. of $10 \mathrm{CSA}(\mathrm{S})$ and $\left.10 \mathrm{CSA}(\mathrm{R}), 25{ }^{\circ} \mathrm{C}\right)$.

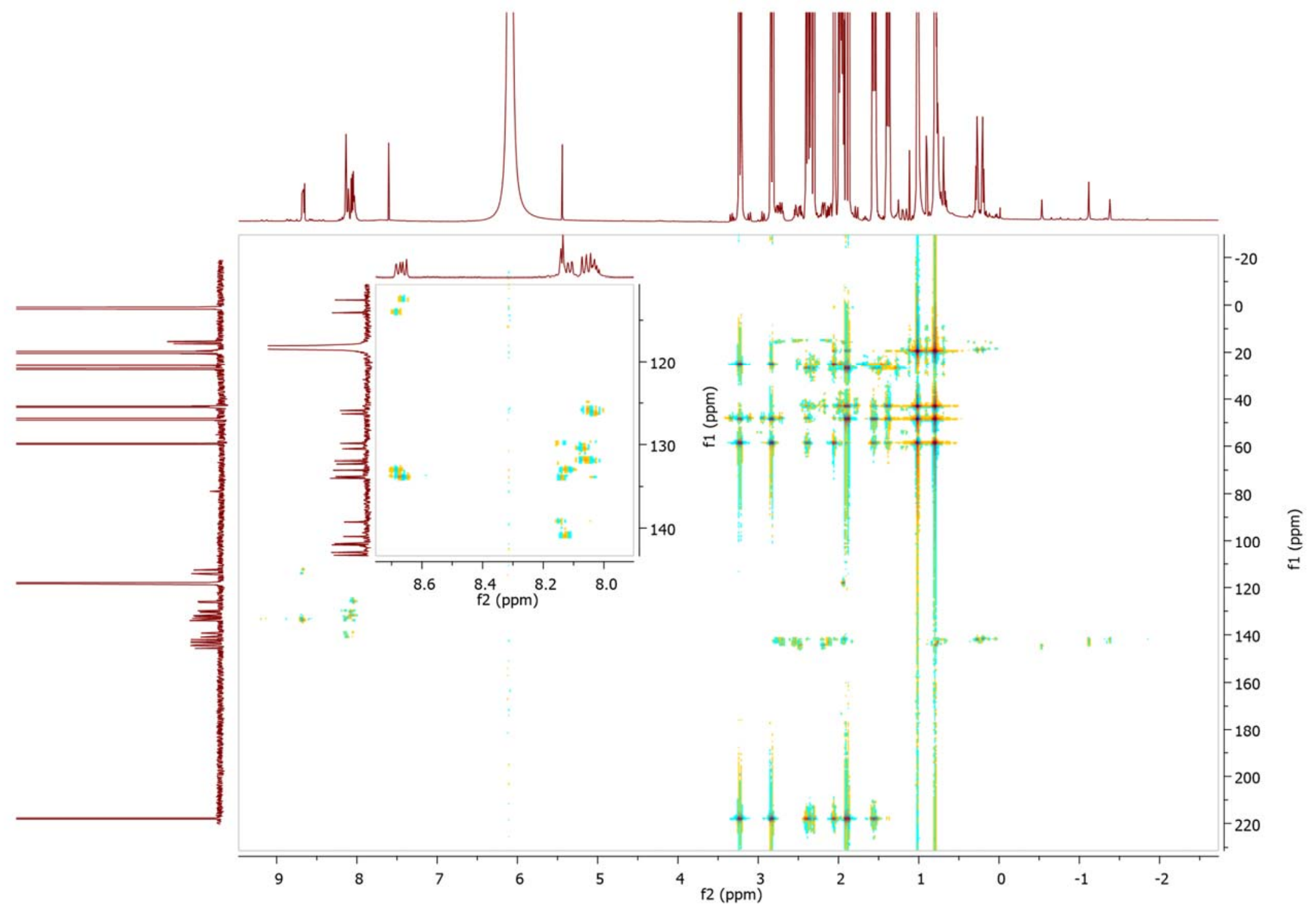




\section{SUPPORTING INFORVATION}

Figure S29. ${ }^{1} \mathrm{H}-{ }^{13} \mathrm{C}$ HMBC spectrum of $\alpha_{2}, \beta_{2}-\mathrm{P} \cdot 10 \mathrm{CSA}(\mathrm{SR})$ with expansion of areas of interest (acetonitrile- $d_{3}, 20$ eq. of $10 \mathrm{CSA}(\mathrm{S})$ and $\left.10 \mathrm{CSA}(\mathrm{R}), 25^{\circ} \mathrm{C}\right)$.

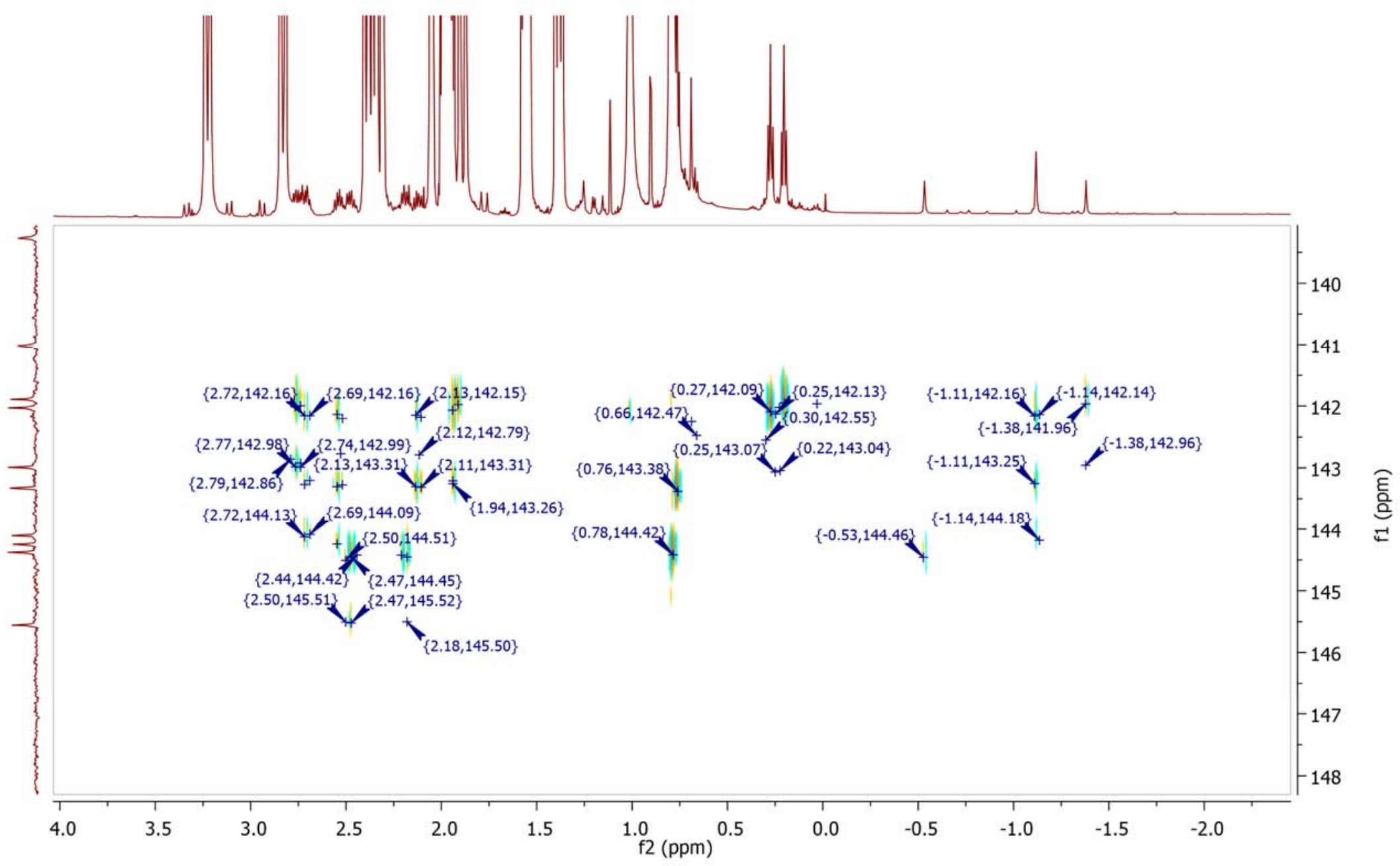

Figure S30. ${ }^{1} \mathrm{H}-{ }^{13} \mathrm{C}$ HMBC spectrum, expansion of area of interest of $\alpha_{2}, \beta_{2}-\mathrm{P} \cdot 10 \mathrm{CSA}(\mathrm{SR})$ (acetonitrile- $d_{3}, 20$ eq. of $10 \mathrm{CSA}(\mathrm{S})$ and $\left.10 \mathrm{CSA}(\mathrm{R}), 25^{\circ} \mathrm{C}\right)$. 


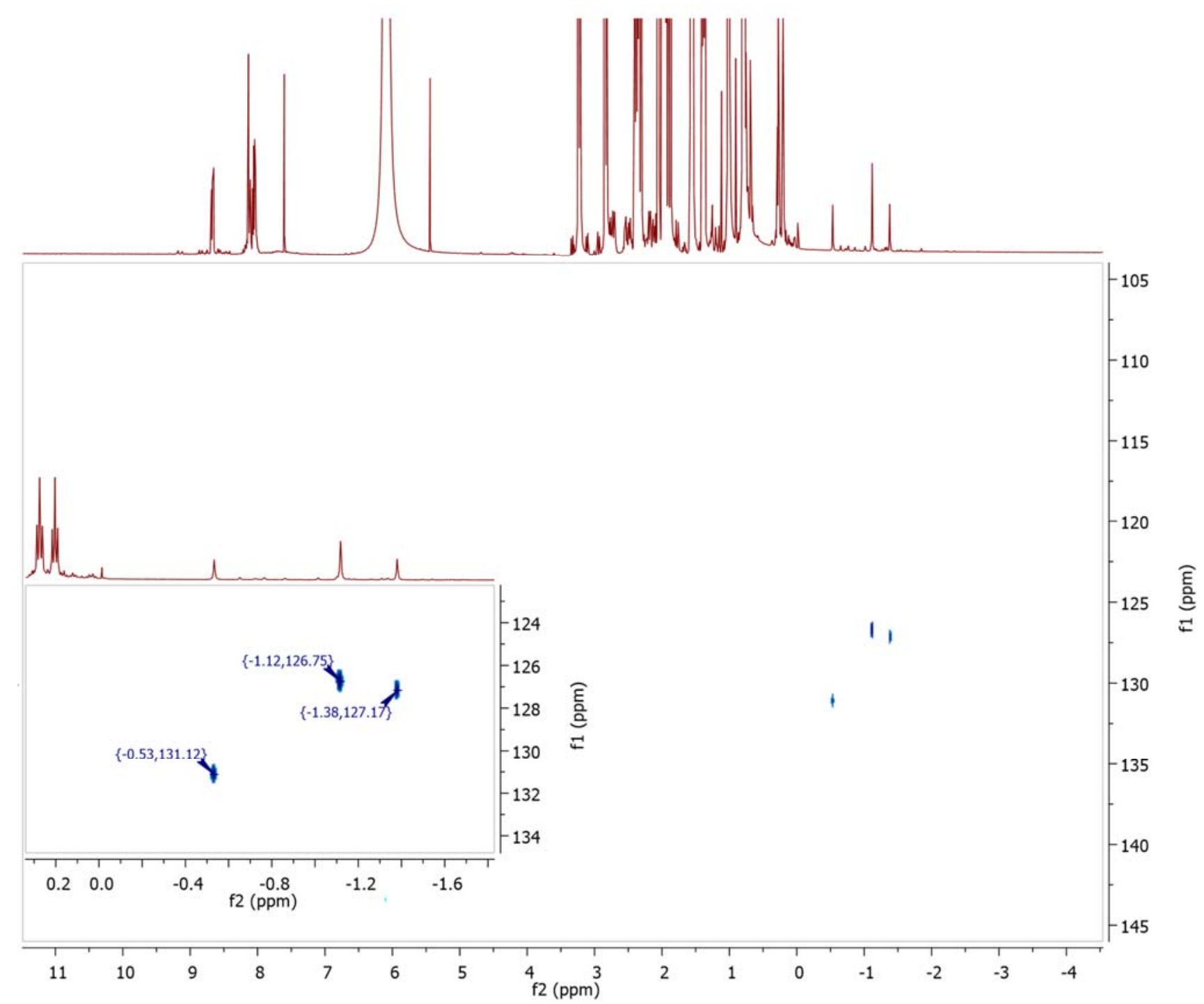

Figure S31. ${ }^{1} \mathrm{H}-{ }^{15} \mathrm{~N}$ HSQC spectrum of $\alpha_{2}, \beta_{2}-\mathrm{P} \cdot 10 \mathrm{CSA}(\mathrm{SR})$ with expansion of areas of interest (acetonitrile- $d_{3}, 20$ eq. of $10 \mathrm{CSA}(\mathrm{S})$ and $\left.10 \mathrm{CSA}(\mathrm{R}), 25^{\circ} \mathrm{C}\right)$ 


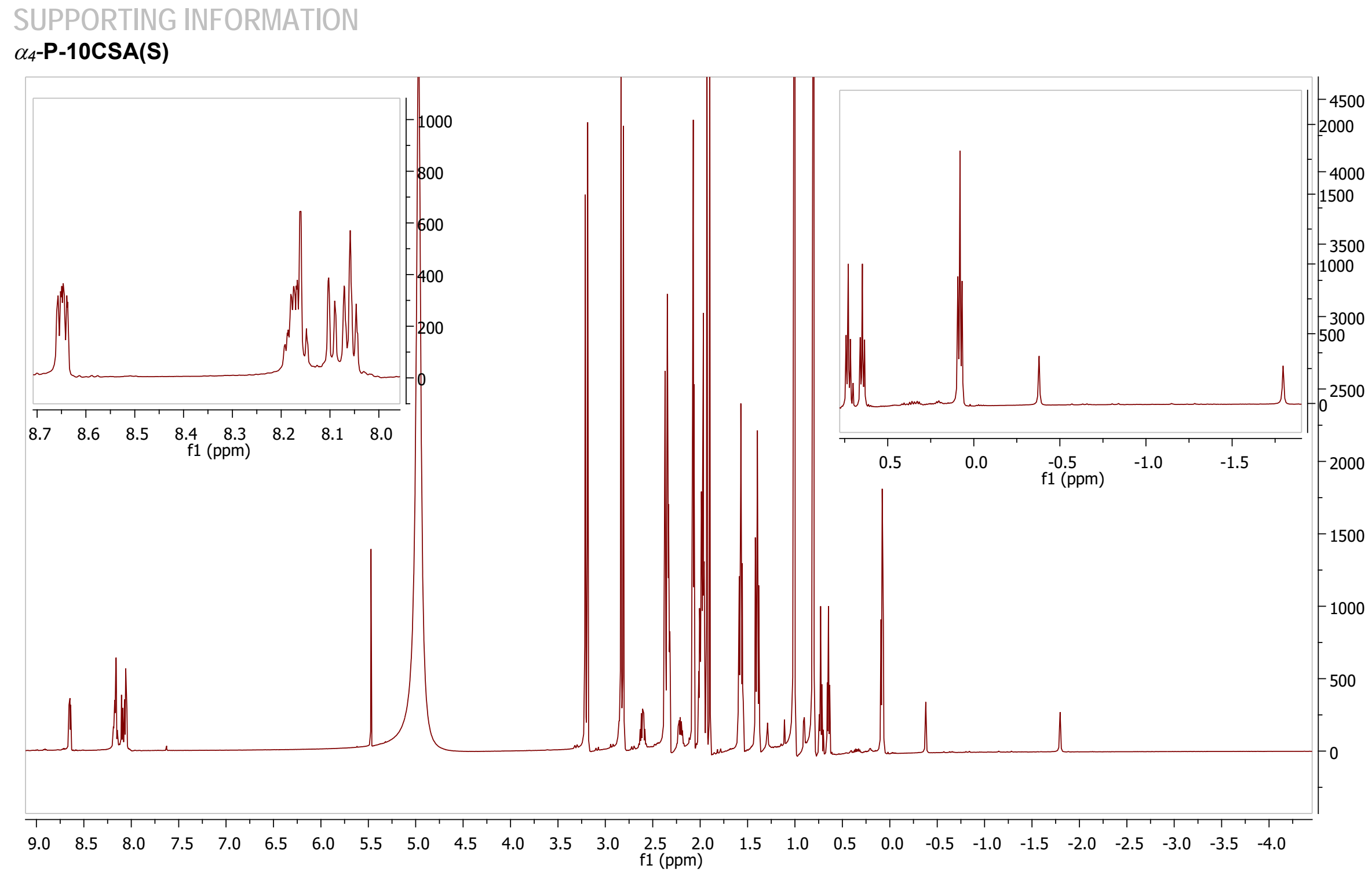

Figure S32. ${ }^{1} \mathrm{H}$ NMR spectrum of $\alpha_{4}-\mathrm{P} \cdot 10 \mathrm{CSA}(\mathrm{S})$ with the expansion of areas of interest (600 MHz, acetonitrile- $d_{3}, 20$ eq. of $\left.10 \mathrm{CSA}(\mathrm{S}), 25^{\circ} \mathrm{C}\right)$. 


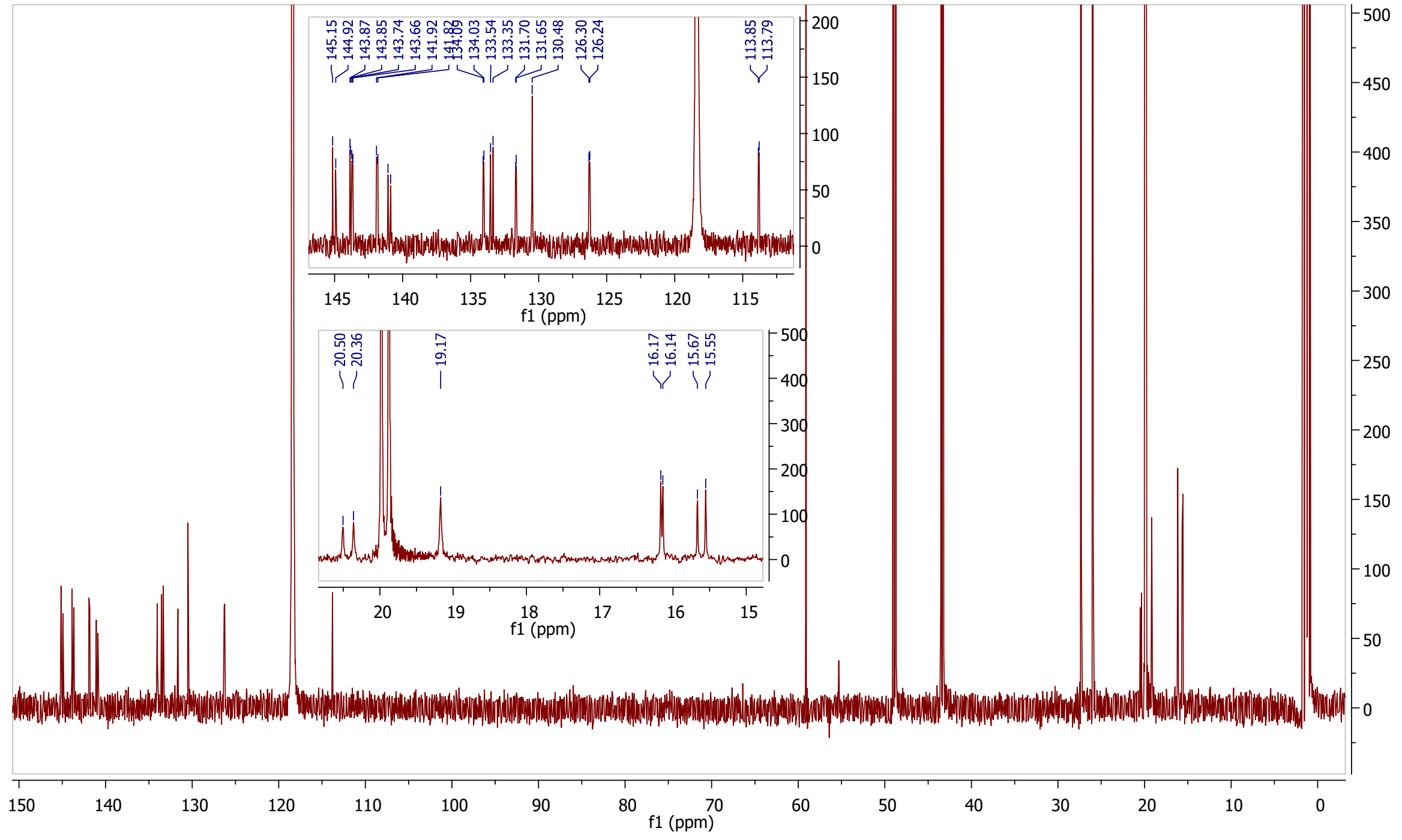

Figure S33. ${ }^{13} \mathrm{C}$ NMR spectrum of $\alpha_{4}-\mathrm{P} \cdot 10 \mathrm{CSA}(\mathrm{S})$ with expansion of areas of interest (151 MHz, acetonitrile- $d_{3}, 20$ eq. of $\left.10 \mathrm{CSA}(\mathrm{S}), 25^{\circ} \mathrm{C}\right)$. 


\section{SUPPORTING INFORMATION}

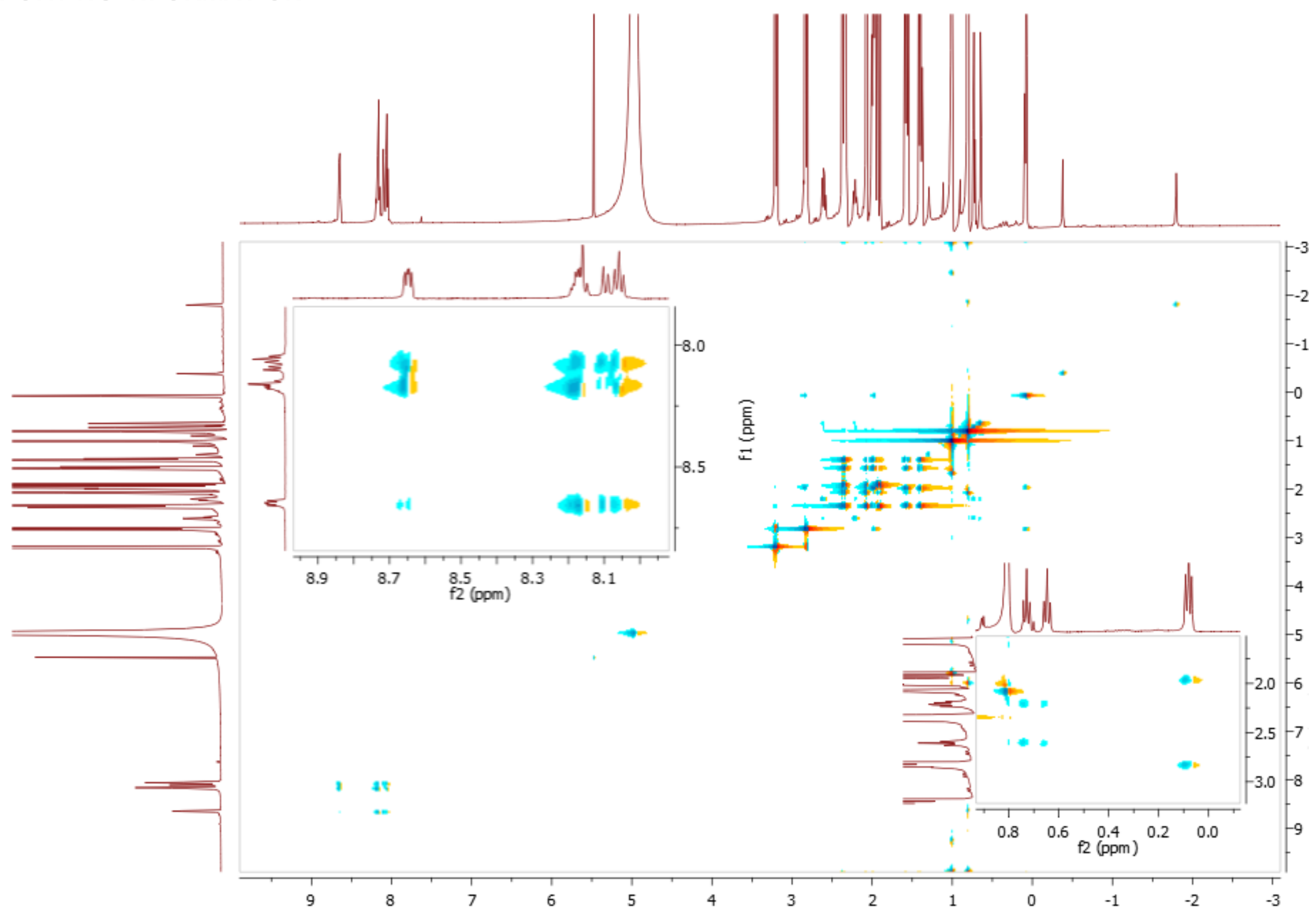

Figure S34. ${ }^{1} \mathrm{H}-{ }^{1} \mathrm{H}$ TOCSY spectrum of $\alpha_{4}-\mathrm{P} \cdot 10 \mathrm{CSA}(\mathbf{S})$ with expansion of areas of interest (acetonitrile- $d_{3}, 20$ eq. of $\left.10 \mathrm{CSA}(\mathrm{S}), 25^{\circ} \mathrm{C}\right)$. 


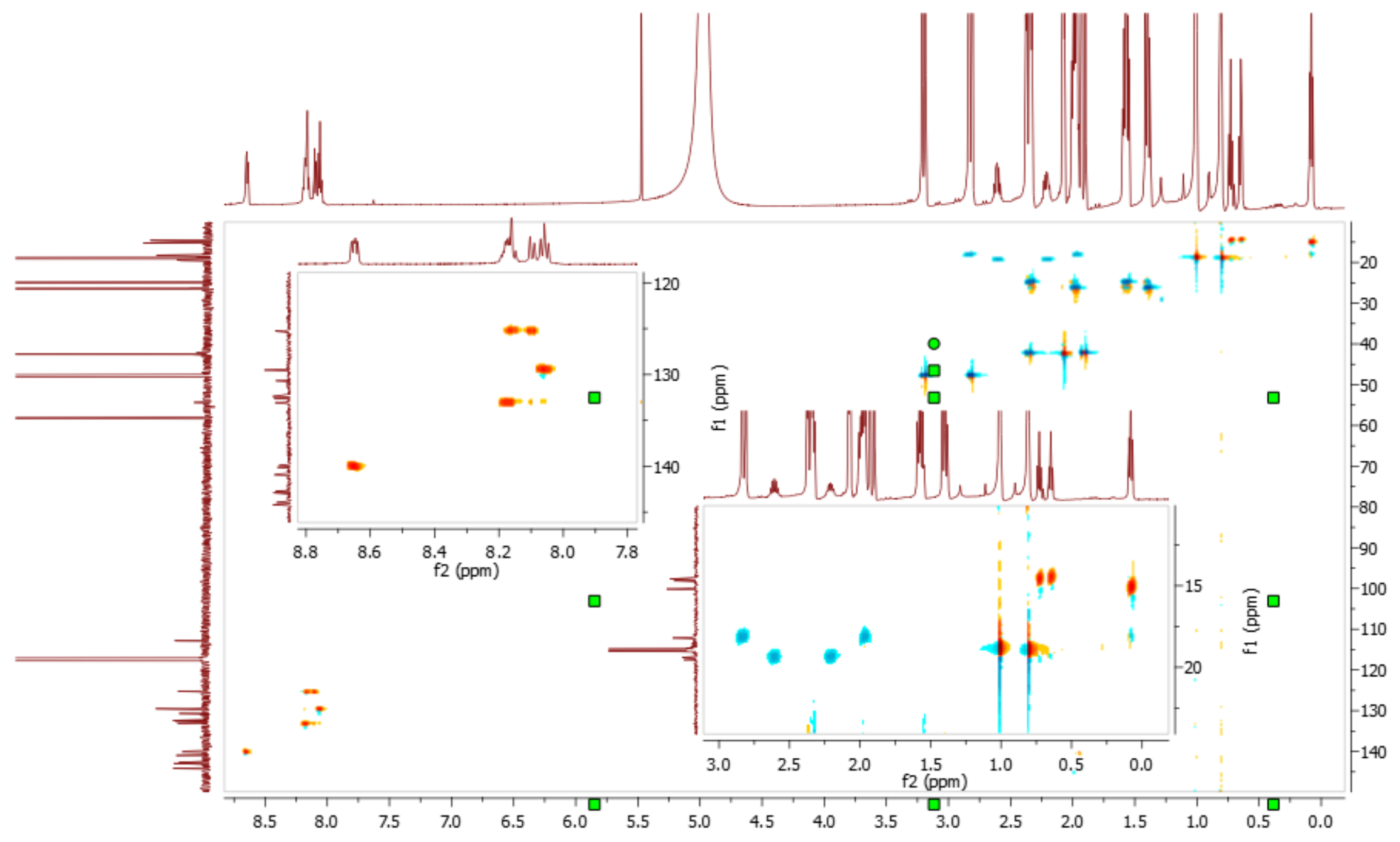

Figure S35. ${ }^{1} \mathrm{H}-{ }^{13} \mathrm{C}$ HSQC spectrum of $\alpha_{4}-\mathrm{P} \cdot 10 \mathrm{CSA}(\mathrm{S})$ with expansion of areas of interest (acetonitrile- $d_{3}, 20$ eq. of $\left.10 \mathrm{CSA}(\mathrm{S}), 25^{\circ} \mathrm{C}\right)$. 


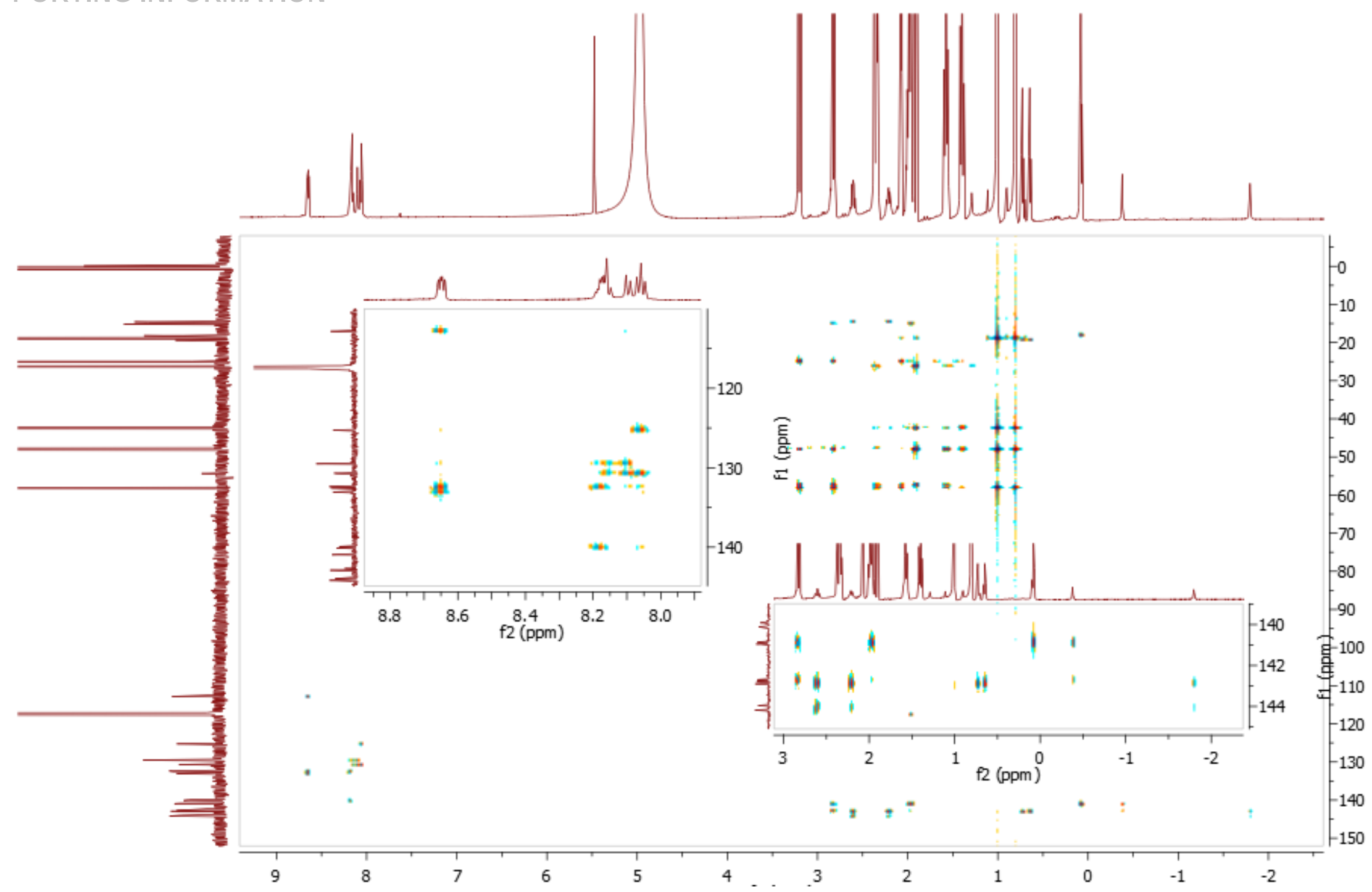

Figure S36. ${ }^{1} \mathrm{H}-{ }^{13} \mathrm{C}$ HMBC spectrum of $\alpha_{4}-\mathbf{P} \cdot 10 \mathrm{CSA}(\mathrm{S})$ with expansion of areas of interest (acetonitrile- $d_{3}, 20$ eq. of $\left.10 \mathrm{CSA}(\mathrm{S}), 25^{\circ} \mathrm{C}\right)$. 


\section{SUPPORTING INFORIATION}
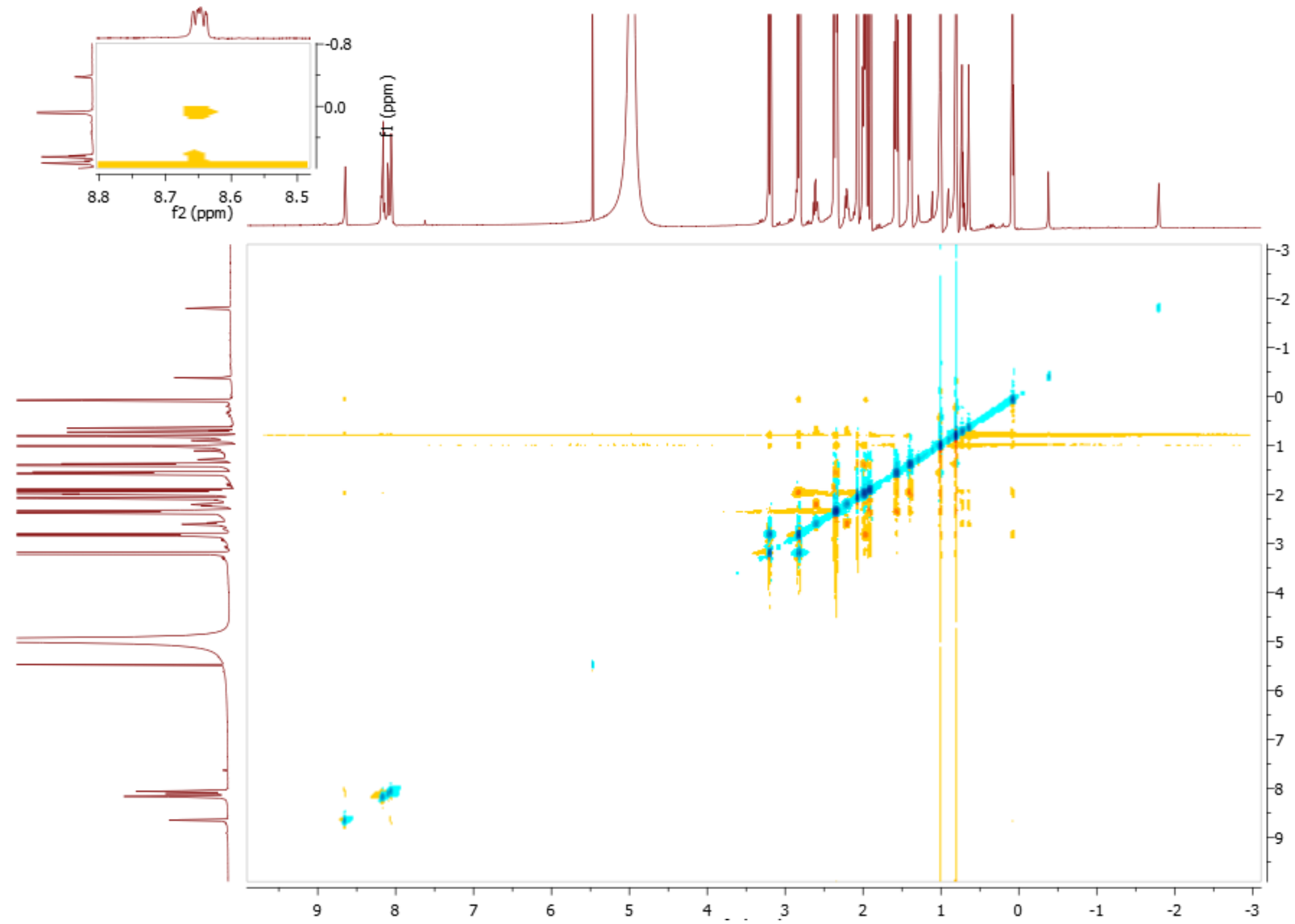


\section{SUPPORTING INFORVATION}

Figure S37. ${ }^{1} \mathrm{H}-{ }^{1} \mathrm{H}$ ROESY spectrum of $\alpha_{4}-\mathrm{P} \cdot 10 \mathrm{CSA}(\mathrm{S})$ with expansion of areas of interest (acetonitrile- $d_{3}, 20$ eq. of $\left.10 \mathrm{CSA}(\mathrm{S}), 25^{\circ} \mathrm{C}\right)$.

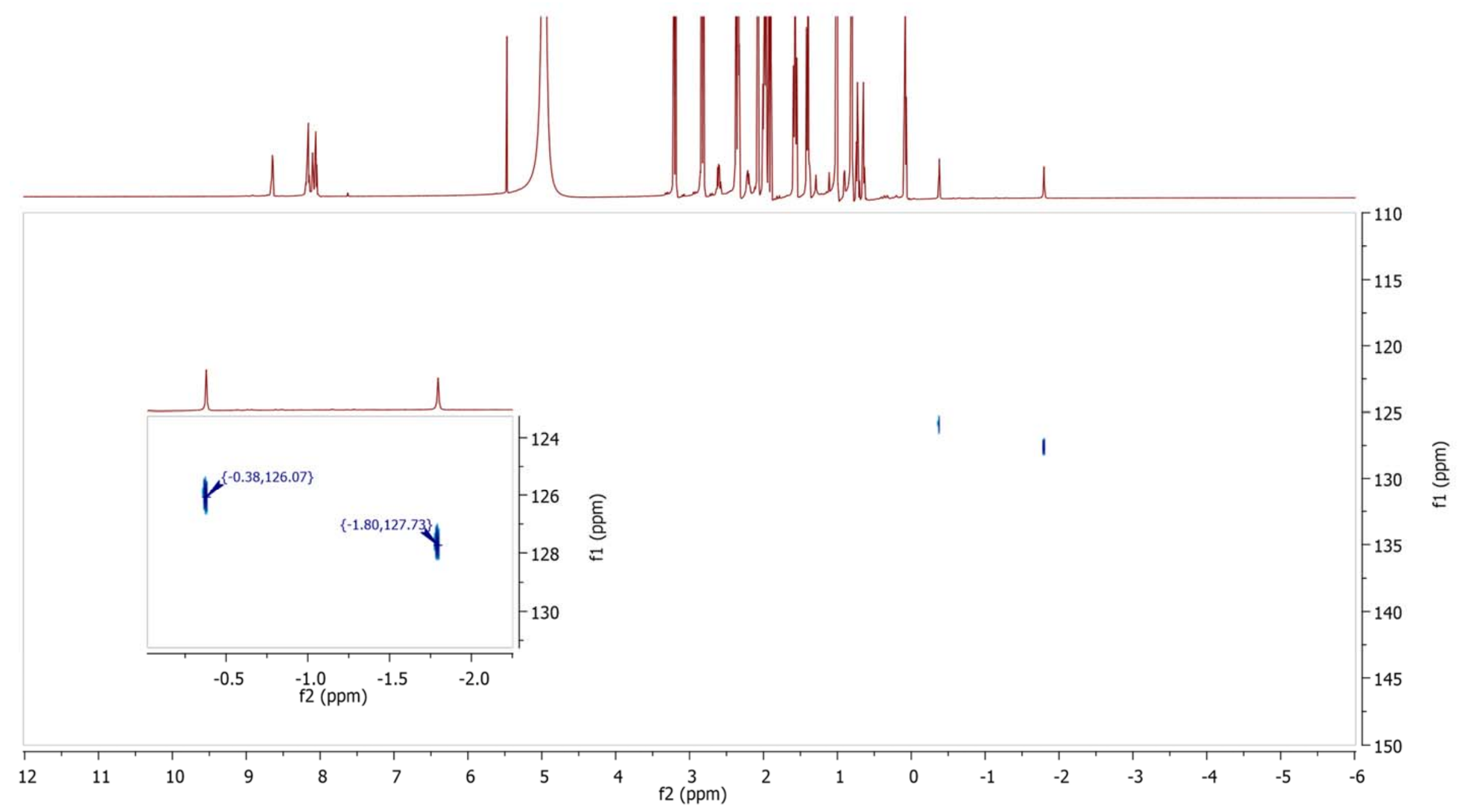

Figure S38. ${ }^{1} \mathrm{H}-{ }^{15} \mathrm{~N} \mathrm{HSQC}$ spectrum of $\alpha_{4}-\mathrm{P} \cdot 10 \mathrm{CSA}(\mathrm{S})$ with expansion of areas of interest (acetonitrile- $d_{3}, 20$ eq. of $\left.10 \mathrm{CSA}(\mathrm{S}), 25^{\circ} \mathrm{C}\right)$. 


\section{SUPPORTING INFORIMATION}

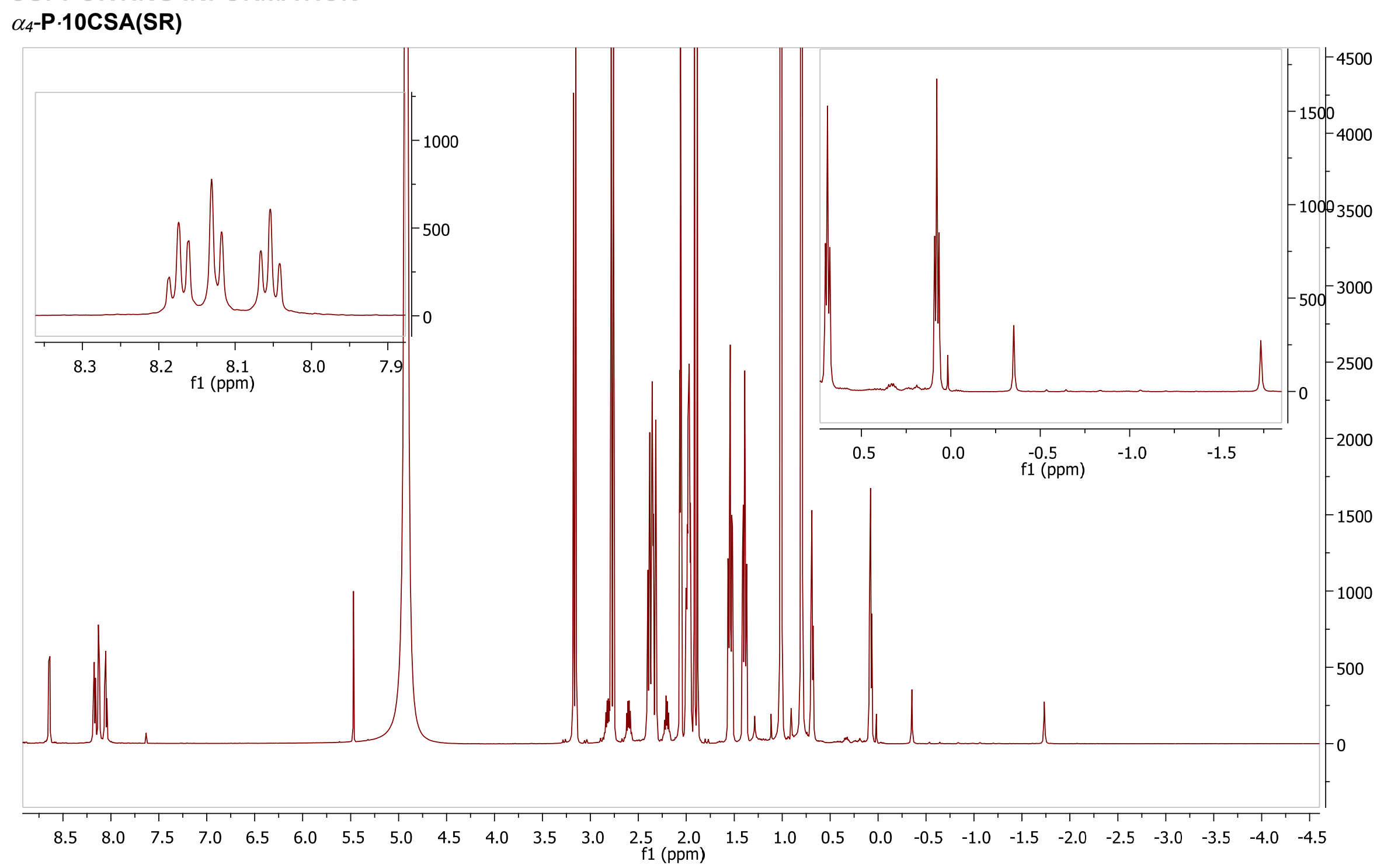

Figure S39. ${ }^{1} \mathrm{H}$ NMR spectrum of $\alpha_{4}-\mathrm{P} \cdot 10 \mathrm{CSA}(\mathrm{SR})$ with expansion of areas of interest $\left(600 \mathrm{MHz}\right.$, acetonitrile- $d_{3}, 20$ eq. of $10 \mathrm{CSA}(\mathrm{S})$ and $\left.10 \mathrm{CSA}(\mathrm{R}), 25^{\circ} \mathrm{C}\right)$. 


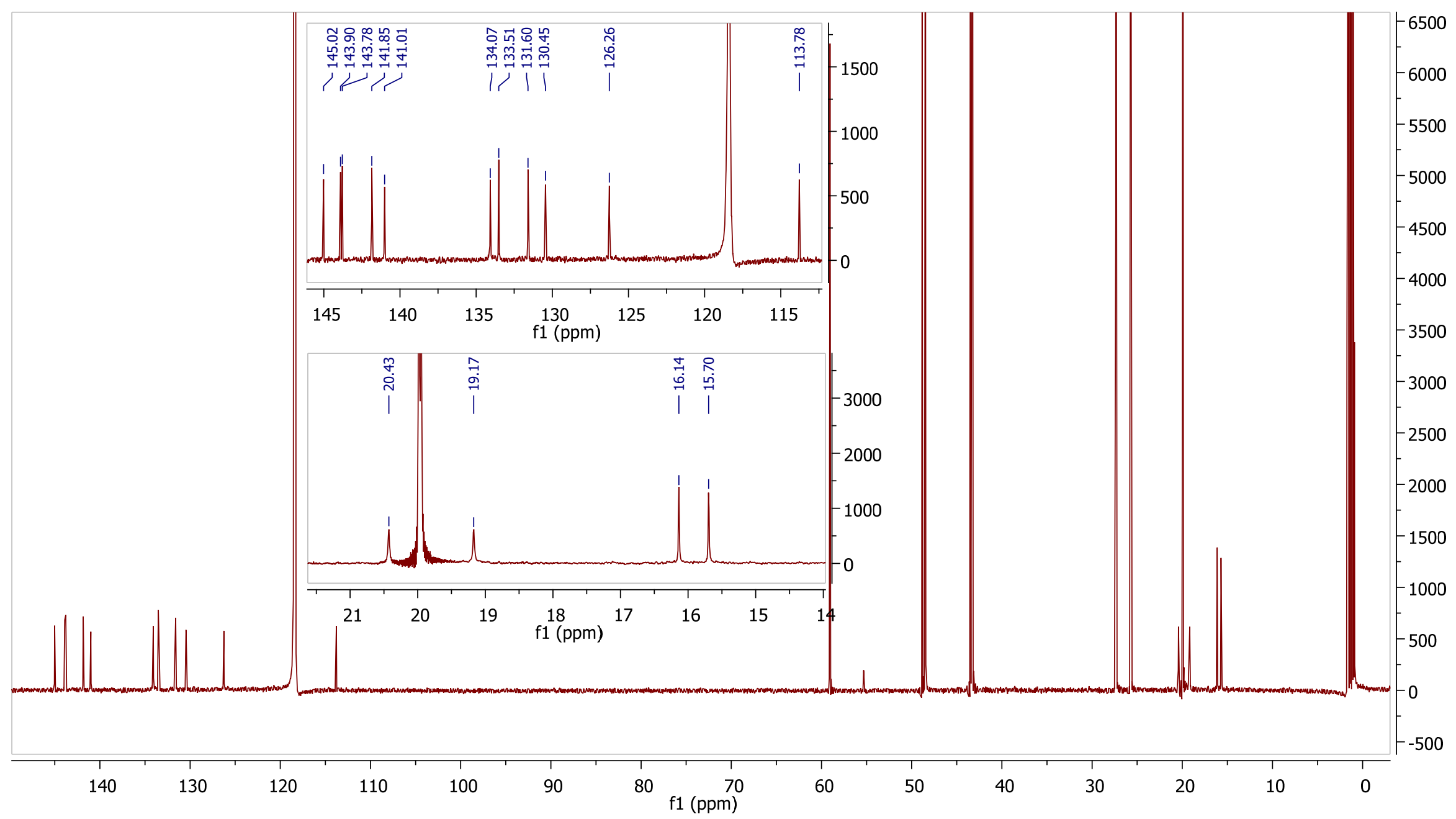

Figure S40. ${ }^{13} \mathrm{C}$ NMR spectrum of $\alpha_{4}-\mathrm{P} \cdot 10 \mathrm{CSA}(\mathrm{SR})$ with expansion of areas of interest (151 MHz, acetonitrile- $d_{3}, 20$ eq. of $10 \mathrm{CSA}(\mathrm{S})$ and $\left.10 \mathrm{CSA}(\mathrm{R}), 25^{\circ} \mathrm{C}\right)$. 


\section{SUPPORTING INFORIMATION}

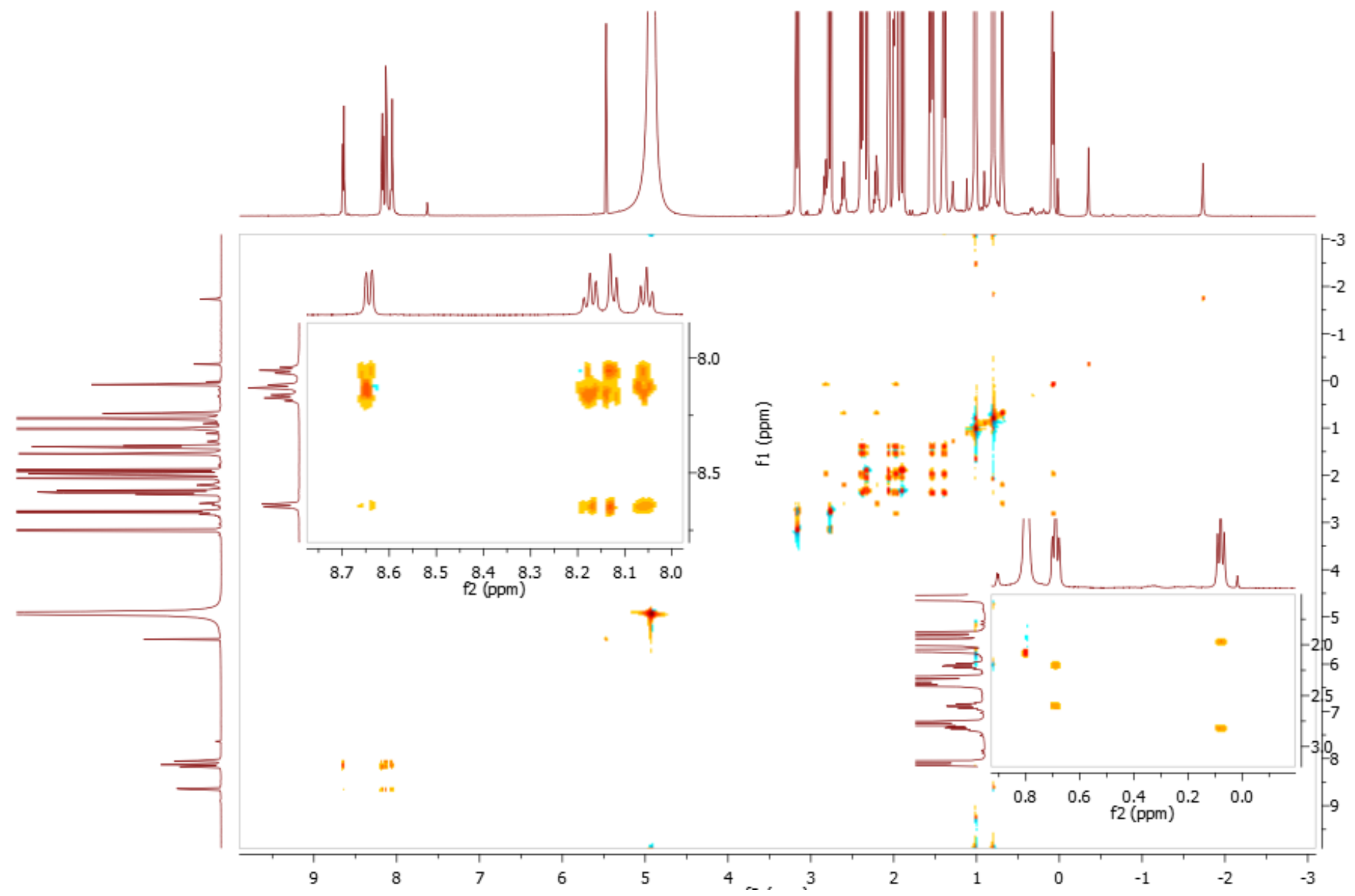

Figure S41. ${ }^{1} \mathrm{H}-{ }^{-1} \mathrm{H}$ TOCSY spectrum of $\alpha_{4}-\mathrm{P} \cdot 10 \mathrm{CSA}(\mathrm{SR})$ with expansion of areas of interest (acetonitrile- $d_{3}, 20$ eq. of $10 \mathrm{CSA}(\mathrm{S})$ and $\left.10 \mathrm{CSA}(\mathrm{R}), 25^{\circ} \mathrm{C}\right)$. 


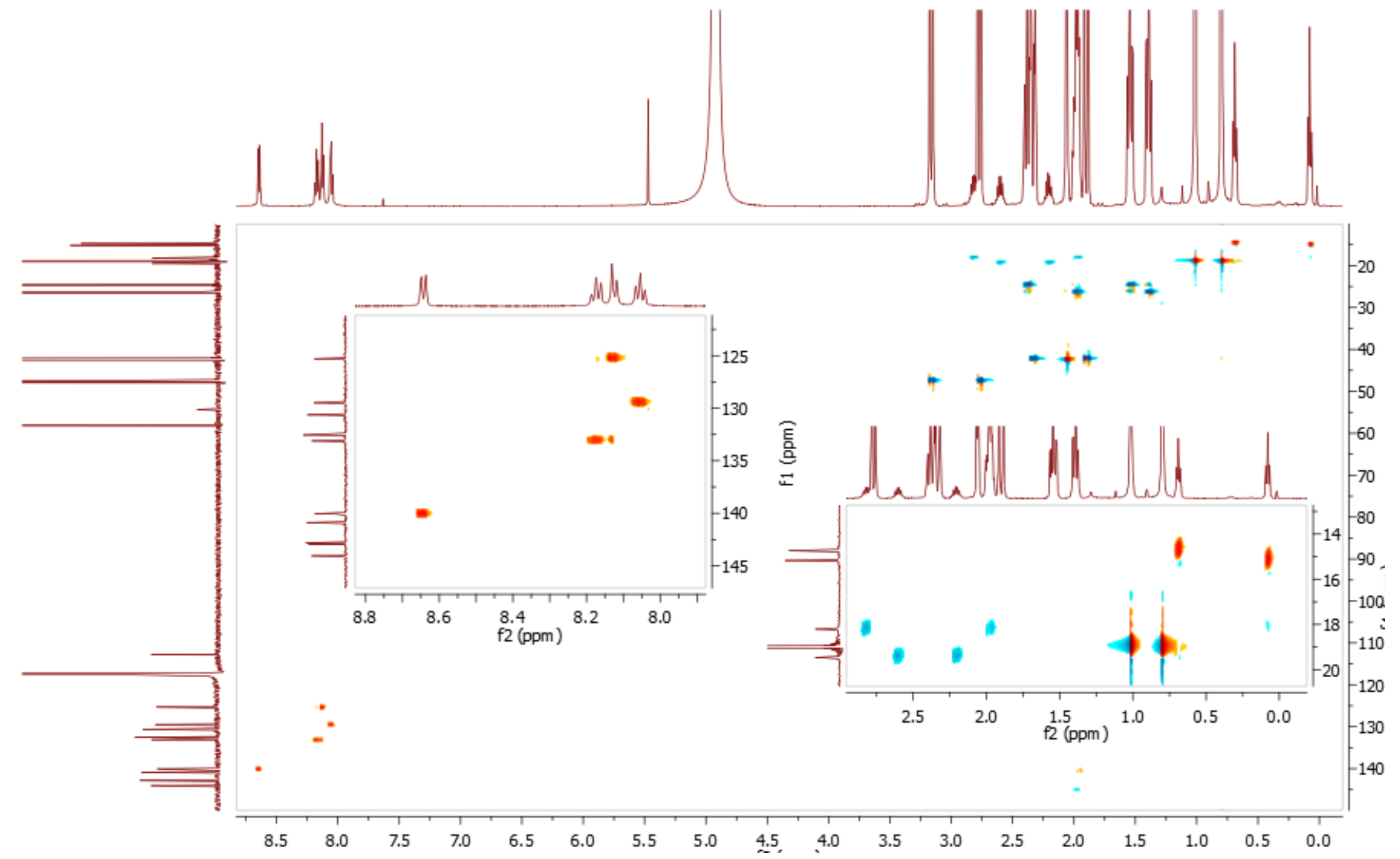

Figure S42. ${ }^{1} \mathrm{H}-{ }^{13} \mathrm{C}$ HSQC spectrum of $\alpha_{4}-\mathrm{P} \cdot 10 \mathrm{CSA}(\mathrm{SR})$ with expansion of areas of interest (acetonitrile- $d_{3}, 20$ eq. of $10 \mathrm{CSA}(\mathrm{S})$ and $\left.10 \mathrm{CSA}(\mathrm{R}), 25{ }^{\circ} \mathrm{C}\right)$. 


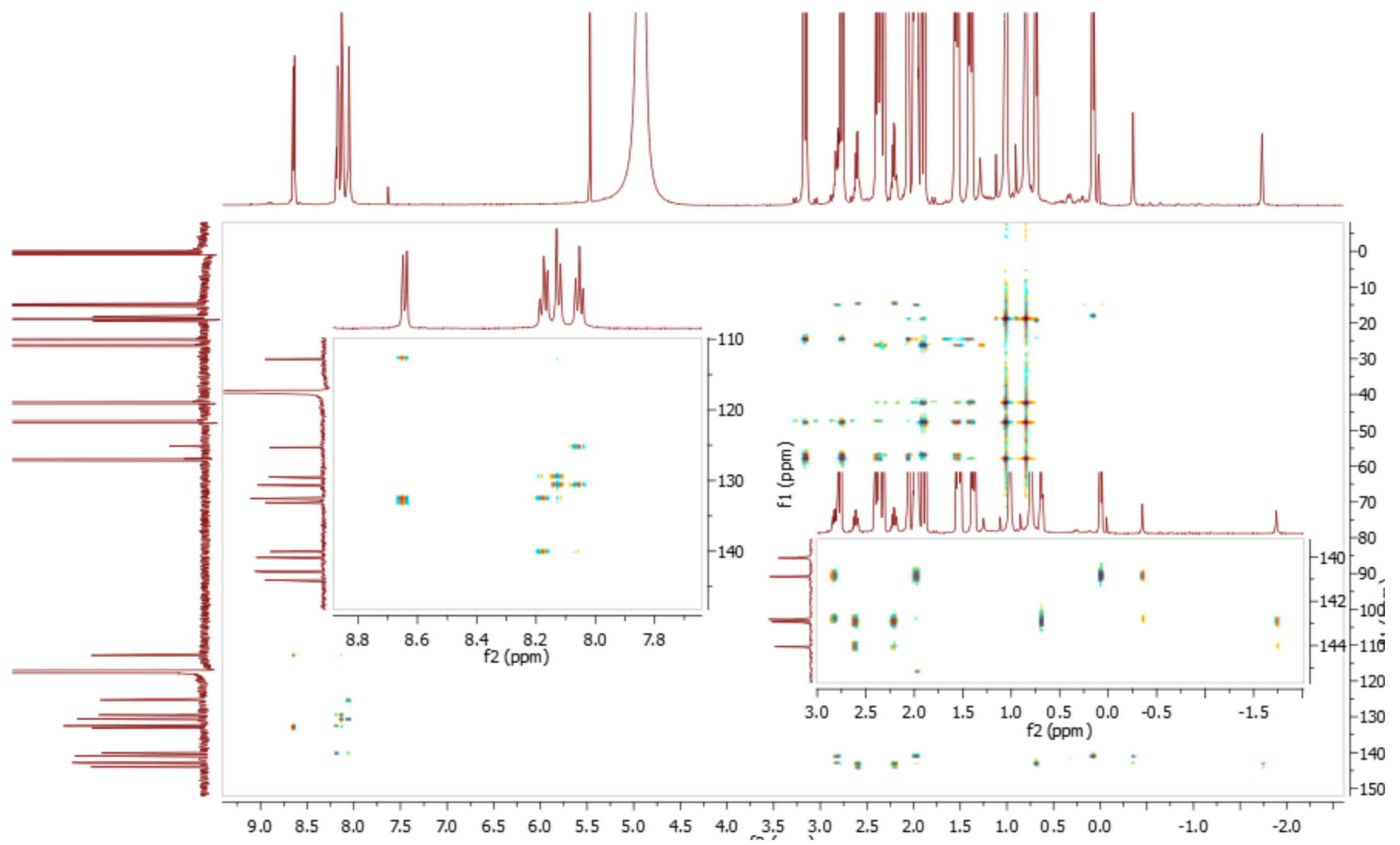

Figure S43. ${ }^{1} \mathrm{H}-{ }^{13} \mathrm{C}$ HMBC spectrum of $\alpha_{4}-\mathrm{P} \cdot 10 \mathrm{CSA}(\mathrm{SR})$ with expansion of areas of interest (acetonitrile- $d_{3}, 20$ eq. of $10 \mathrm{CSA}(\mathrm{S})$ and $\left.10 \mathrm{CSA}(\mathrm{R}), 25^{\circ} \mathrm{C}\right)$. 


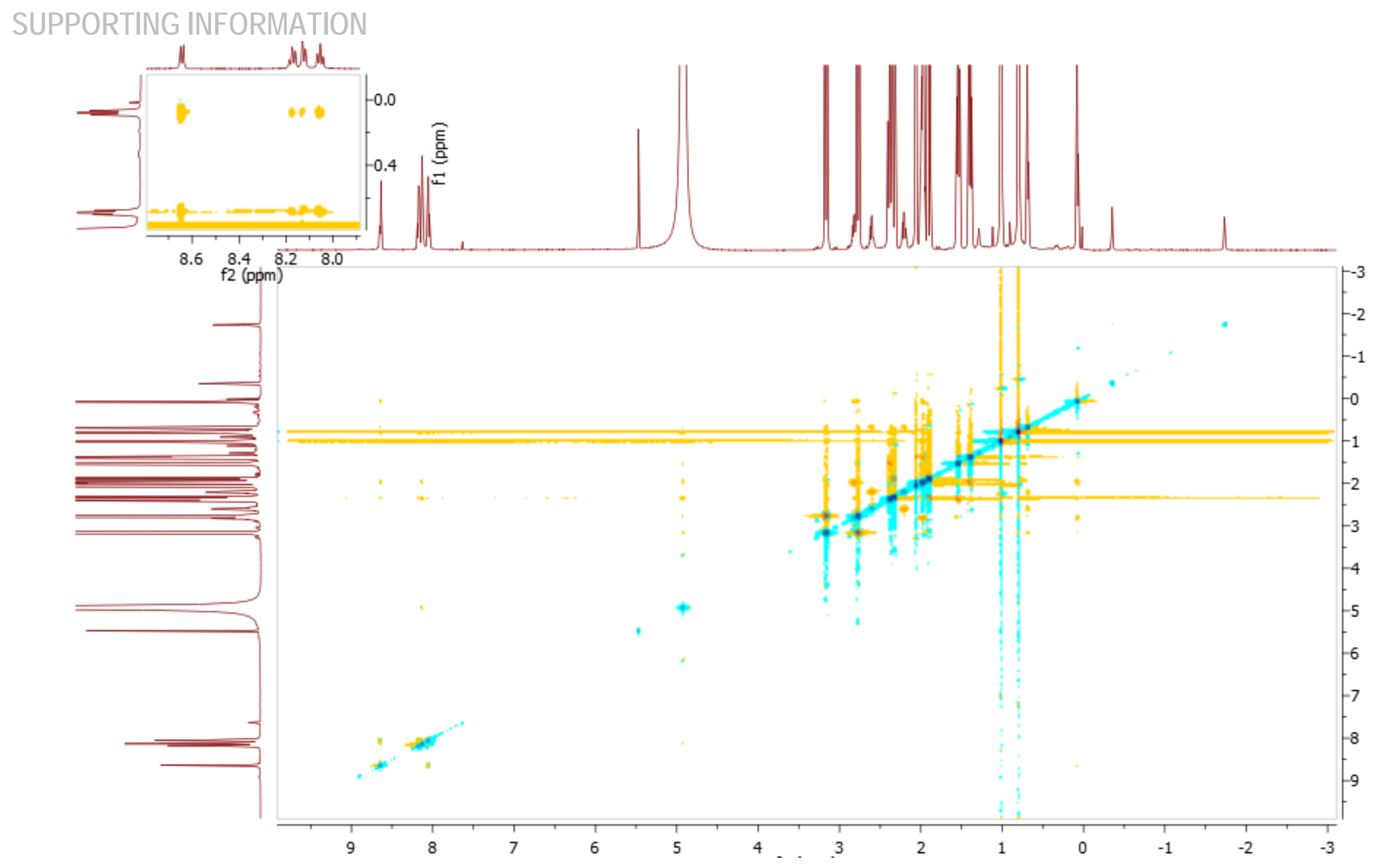

Figure S44. ${ }^{1} \mathrm{H}-{ }^{1} \mathrm{H}$ ROESY spectrum of $\alpha_{4}-\mathrm{P} \cdot 10 \mathrm{CSA}(\mathrm{SR})$ with expansion of areas of interest (acetonitrile- $d_{3}, 20$ eq. of $10 \mathrm{CSA}(\mathrm{S})$ and $\left.10 \mathrm{CSA}(\mathrm{R}), 25^{\circ} \mathrm{C}\right)$. 


\section{SUPPORTING INFORIMTION}

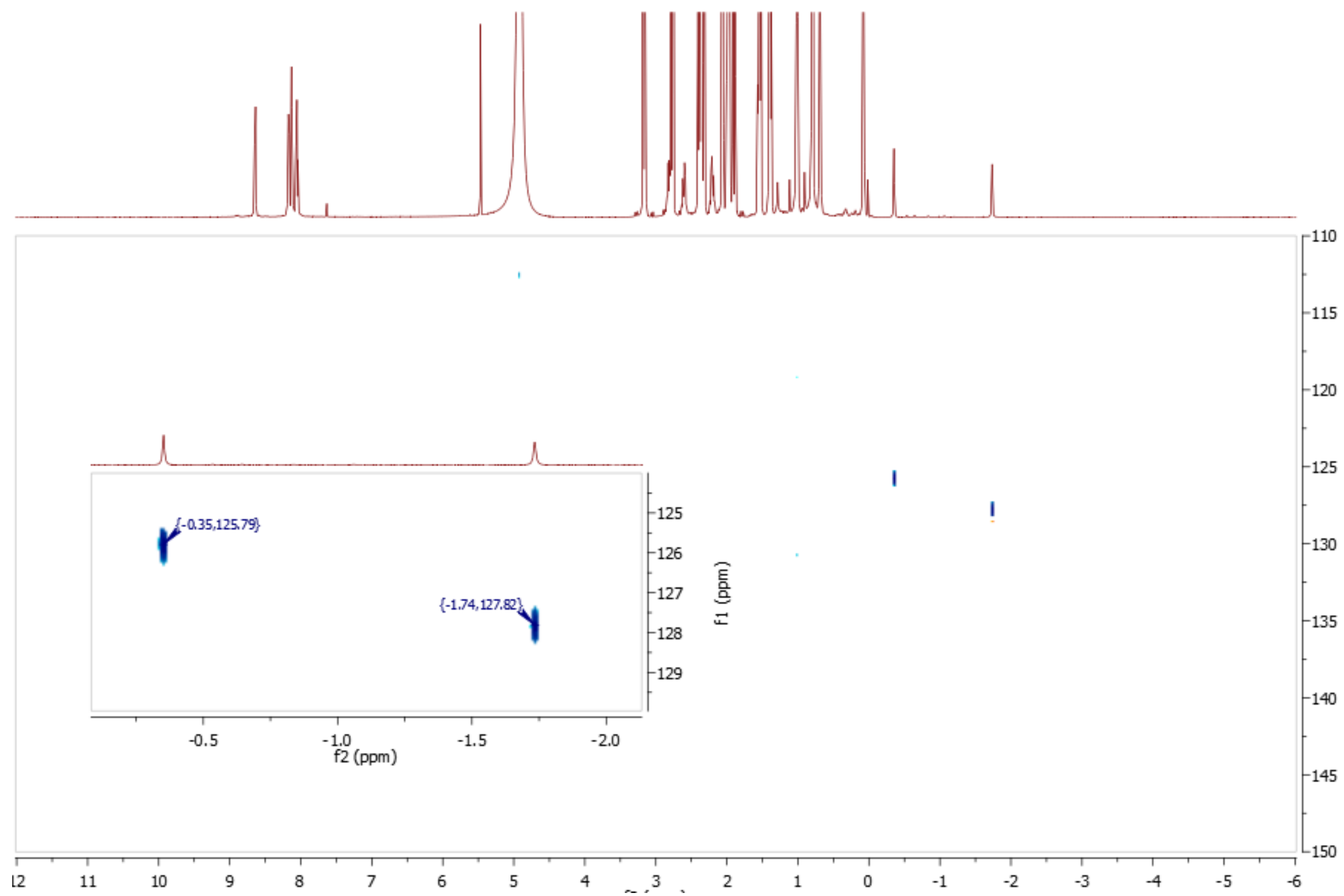

Figure S45. ${ }^{1} \mathrm{H}-{ }^{15} \mathrm{~N}$ HSQC spectrum of $\alpha_{4}-\mathrm{P} \cdot 10 \mathrm{CSA}(\mathrm{SR})$ with expansion of areas of interest (acetonitrile- $d_{3}, 20$ eq. of $10 \mathrm{CSA}(\mathrm{S})$ and $\left.10 \mathrm{CSA}(\mathrm{R}), 25^{\circ} \mathrm{C}\right)$ 
$\alpha \beta \alpha \beta$-P.10CSA(S)

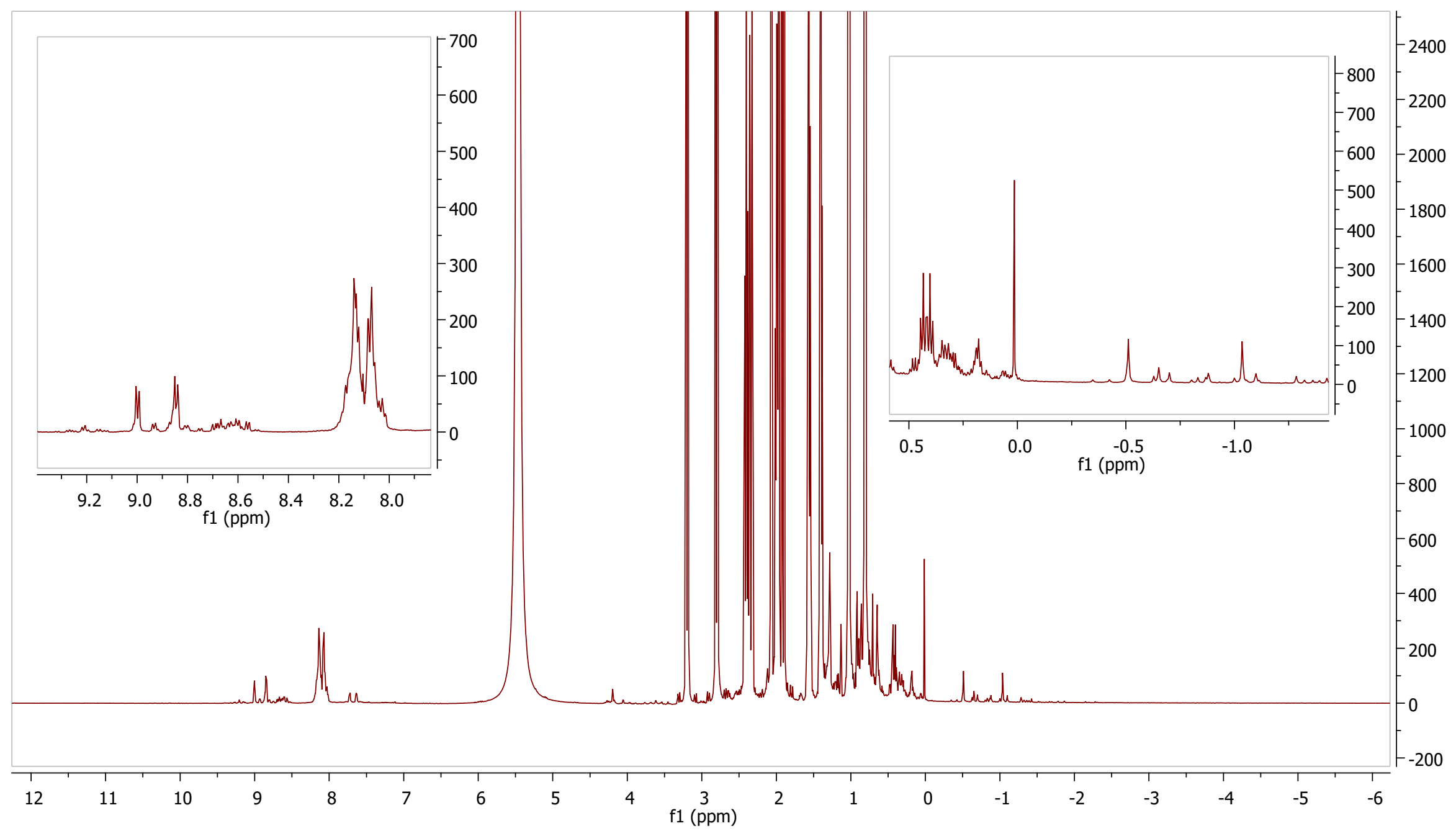

Figure S46. ${ }^{1} \mathrm{H}$ NMR spectrum of $\alpha \beta \alpha \beta-\mathrm{P} \cdot 10 \mathrm{CSA}(\mathrm{S})$ with the expansion of areas of interest $\left(600 \mathrm{MHz}\right.$, acetonitrile- $d_{3}, 20$ eq. of $\left.10 \mathrm{CSA}(\mathrm{S}), 25^{\circ} \mathrm{C}\right)$. 


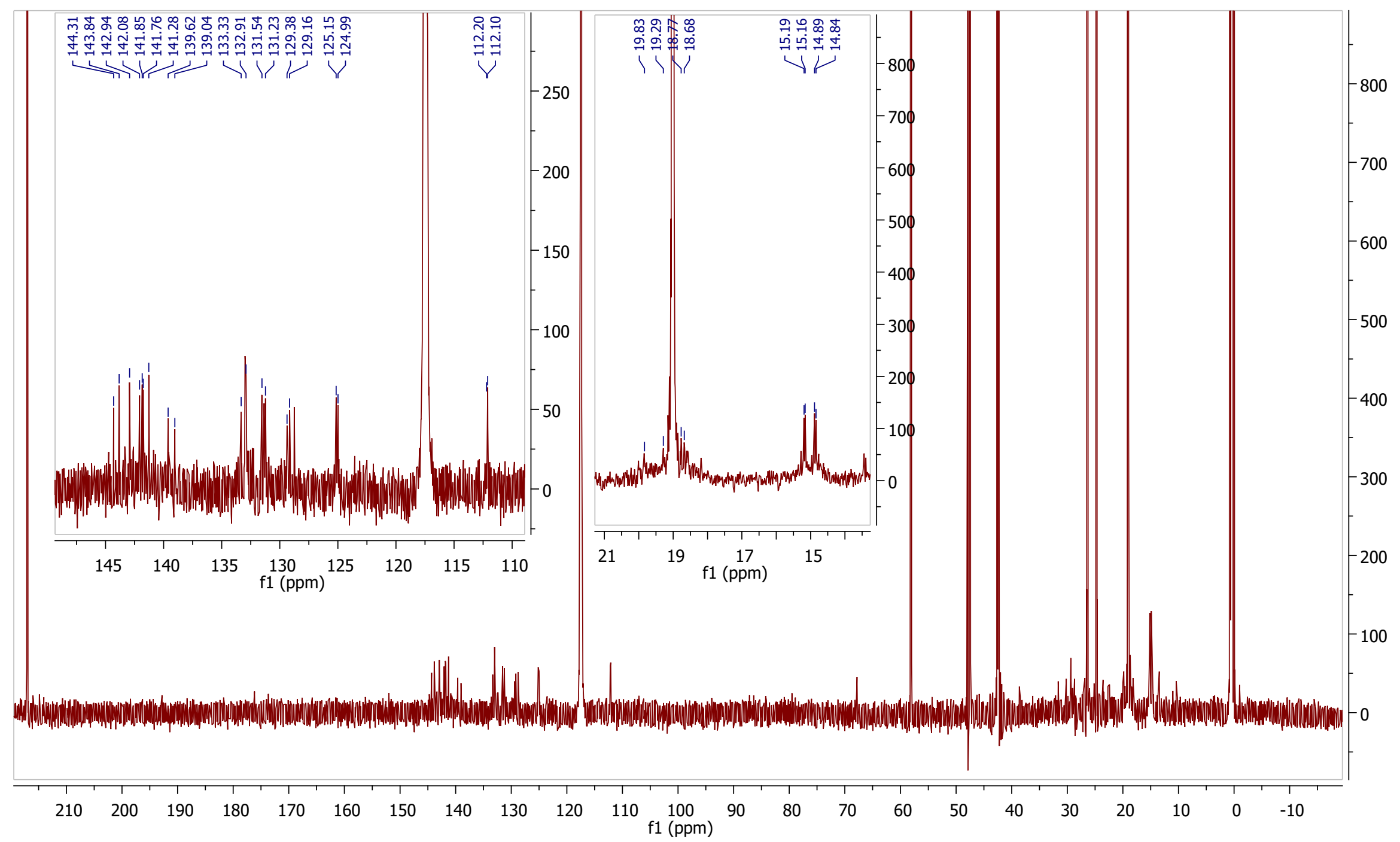

Figure S47. ${ }^{13} \mathrm{C}$ NMR spectrum of $\alpha \beta \alpha \beta-P \cdot 10 C S A(S)$ with expansion of areas of interest (151 MHz, acetonitrile- $d_{3}, 20$ eq. of $\left.10 \mathrm{CSA}(\mathrm{S}), 25^{\circ} \mathrm{C}\right)$. 


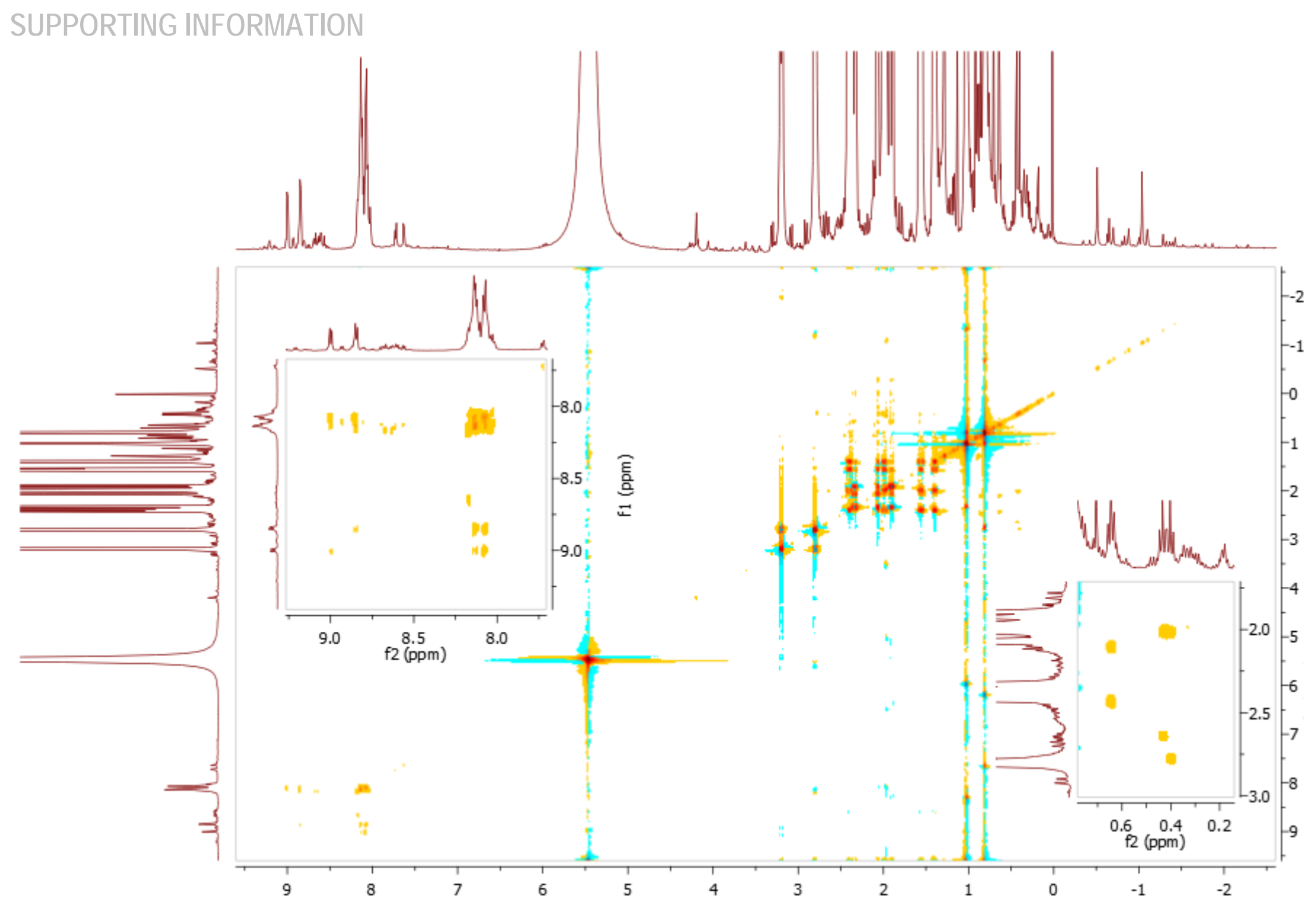

Figure S48. ${ }^{1} \mathrm{H}-{ }^{1} \mathrm{H}$ TOCSY spectrum of $\alpha \beta \alpha \beta-\mathbf{P} \cdot 10 \mathrm{CSA}(\mathbf{S})$ with expansion of areas of interest (acetonitrile- $d_{3}, 20$ eq. of $\left.10 \mathrm{CSA}(\mathrm{S}), 25^{\circ} \mathrm{C}\right)$. 


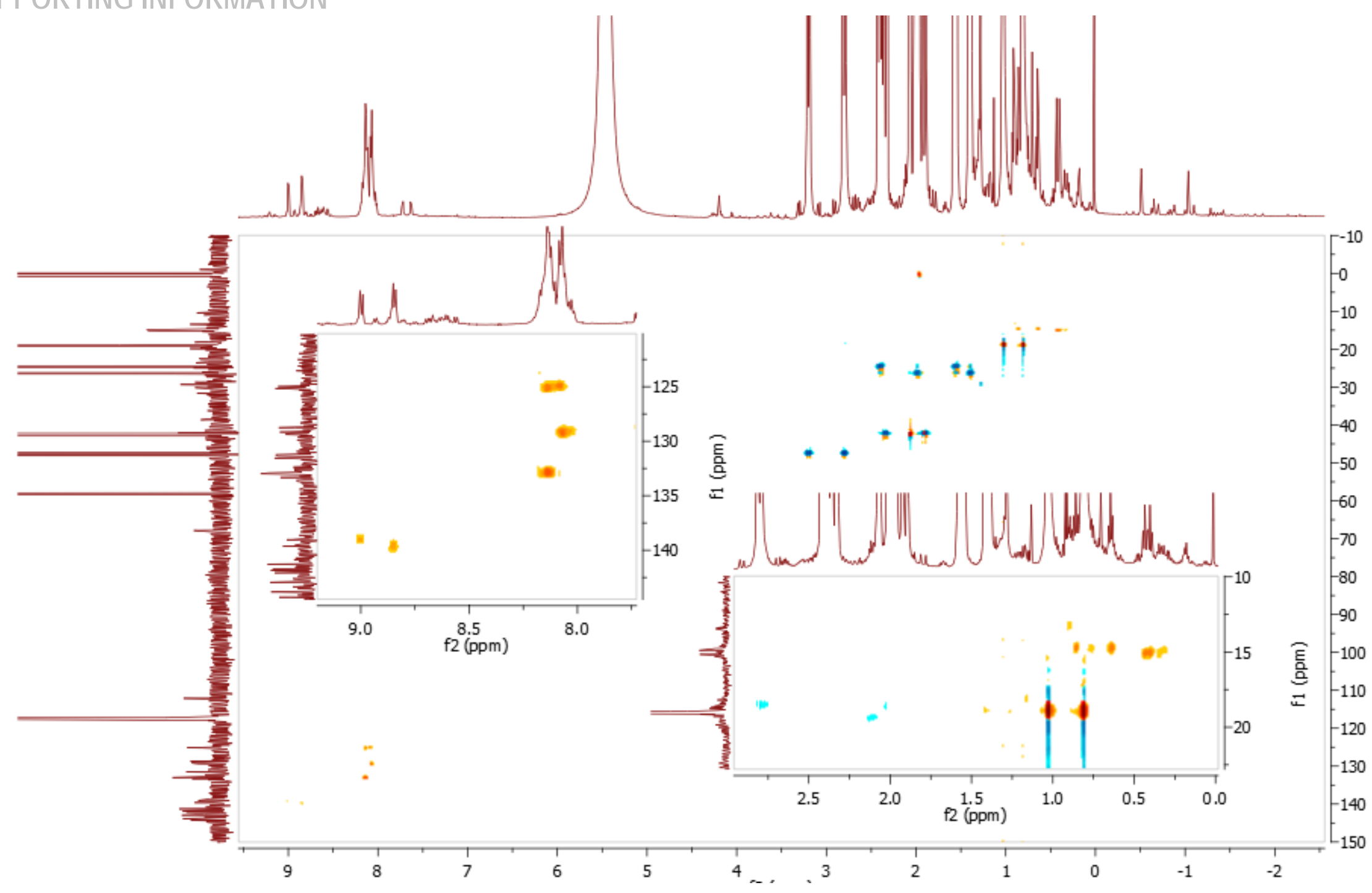

Figure S49. ${ }^{1} \mathrm{H}-{ }^{13} \mathrm{C}$ HSQC spectrum of $\alpha \beta \alpha \beta-\mathrm{P} \cdot 10 \mathrm{CSA}(\mathrm{S})$ with expansion of areas of interest (acetonitrile- $d_{3}, 20$ eq. of $\left.10 \mathrm{CSA}(\mathrm{S}), 25^{\circ} \mathrm{C}\right)$. 


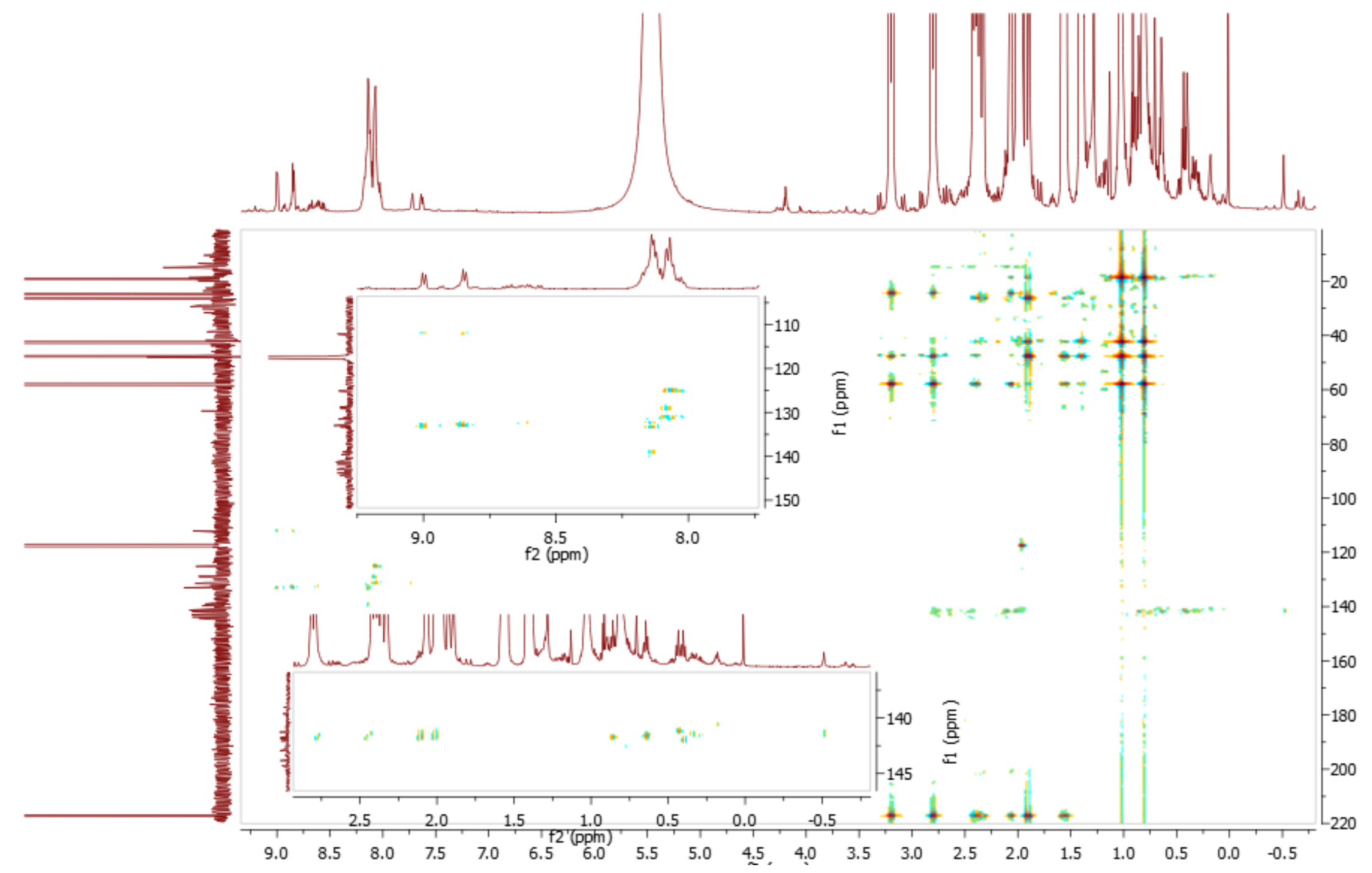

Figure S50. ${ }^{1} \mathrm{H}-{ }^{13} \mathrm{C} H M B C$ spectrum of $\alpha \beta \alpha \beta-\mathbf{P} \cdot 10 \mathrm{CSA}(\mathrm{S})$ with expansion of areas of interest (acetonitrile- $d_{3}, 20$ eq. of $\left.10 \mathrm{CSA}(\mathrm{S}), 25^{\circ} \mathrm{C}\right)$. 


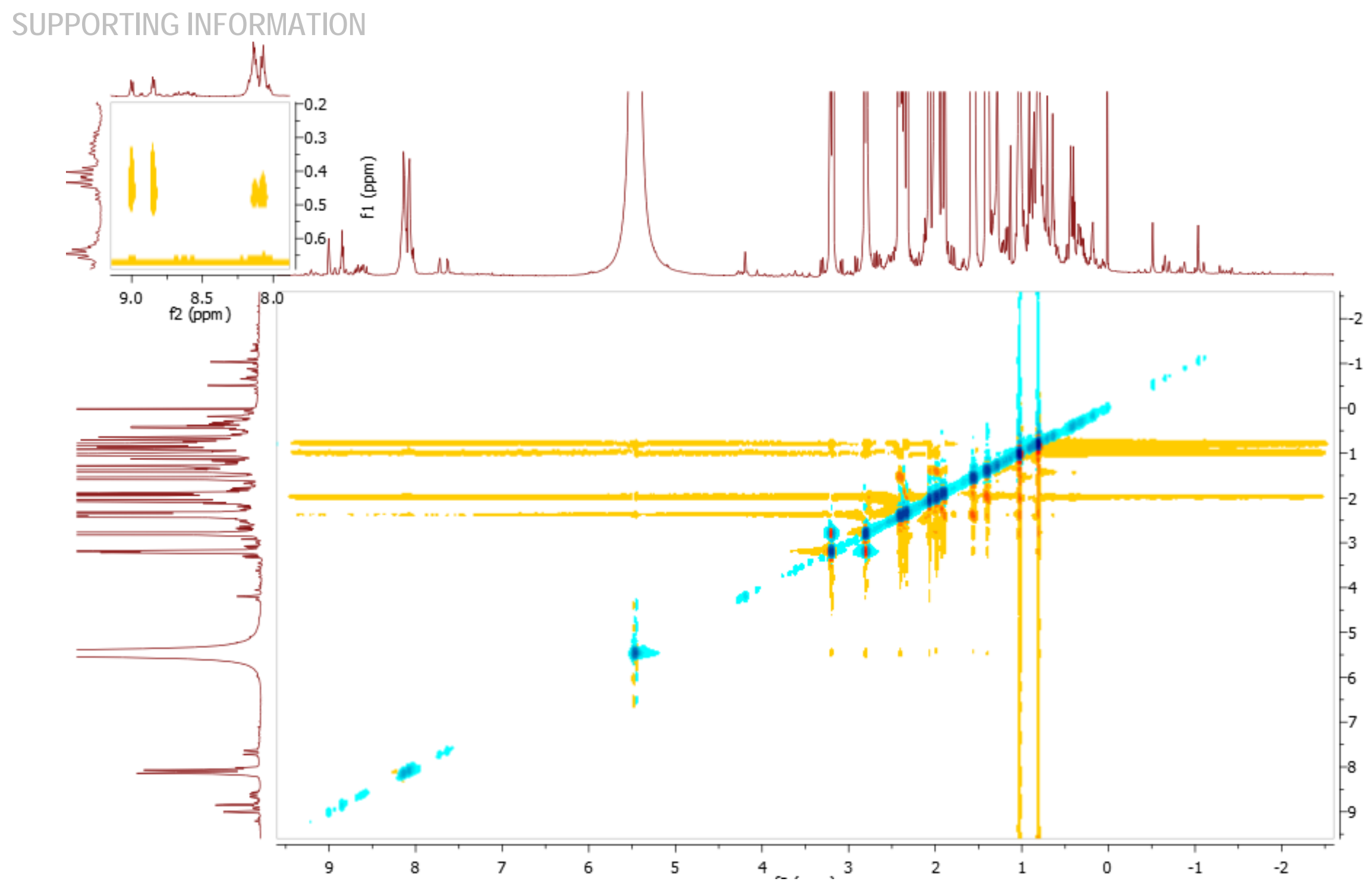

Figure S51. ${ }^{1} \mathrm{H}-{ }^{1} \mathrm{H}$ ROESY spectrum of $\alpha \beta \alpha \beta-\mathrm{P} \cdot 10 \mathrm{CSA}(\mathrm{S})$ with expansion of areas of interest (acetonitrile- $d_{3}, 20$ eq. of $\left.10 \mathrm{CSA}(\mathrm{S}), 25^{\circ} \mathrm{C}\right)$. 


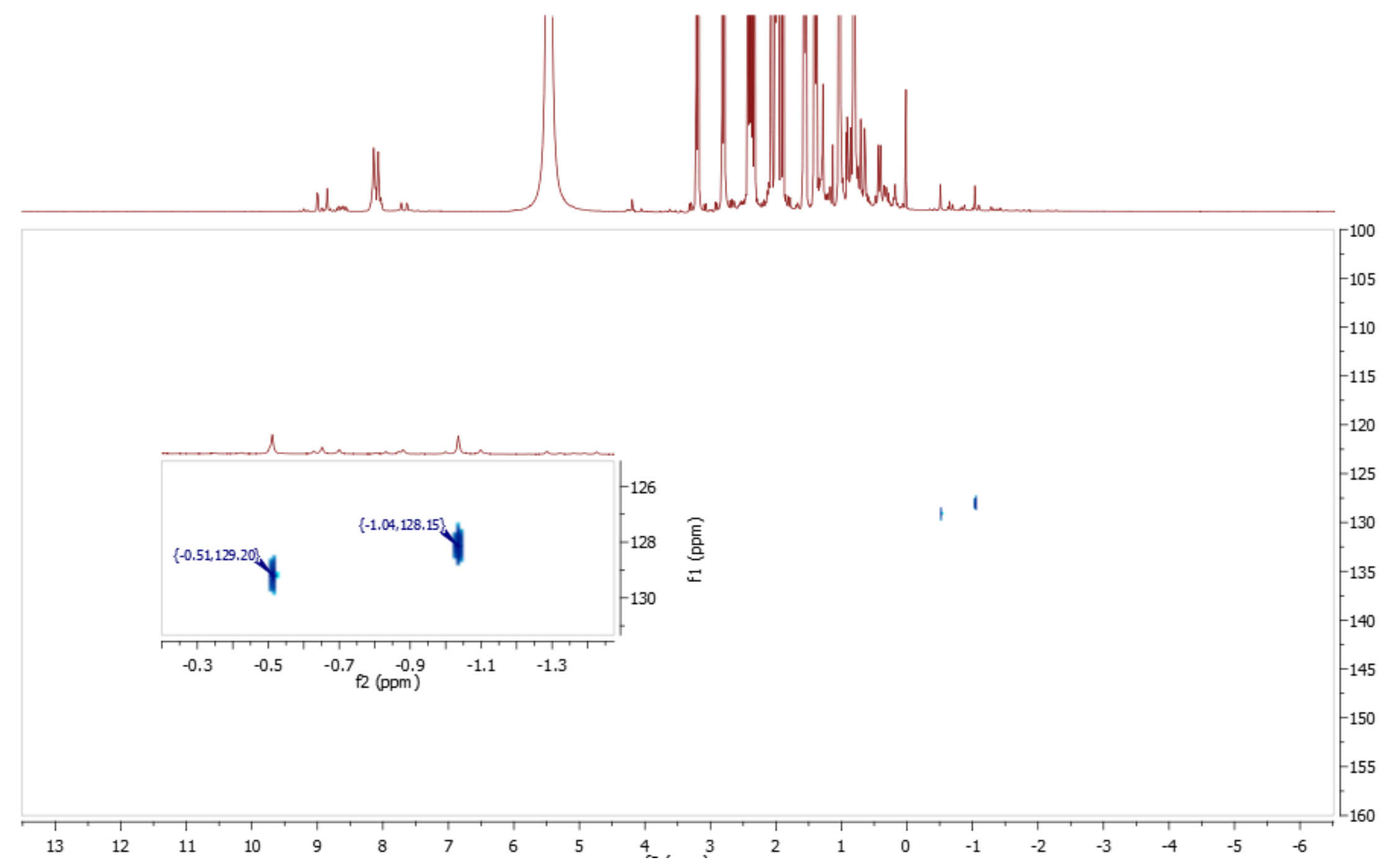

Figure S52. ${ }^{1} \mathrm{H}-{ }^{15} \mathrm{~N}$ HSQC spectrum of $\alpha \beta \alpha \beta-\mathrm{P} \cdot 10 \mathrm{CSA}(\mathrm{S})$ with expansion of areas of interest (acetonitrile- $d_{3}, 20$ eq. of $\left.10 \mathrm{CSA}(\mathrm{S}), 25^{\circ} \mathrm{C}\right)$. 
$\alpha \beta \alpha \beta \cdot \mathbf{P} \cdot \mathbf{1 0 C S A}(\mathbf{S R})$

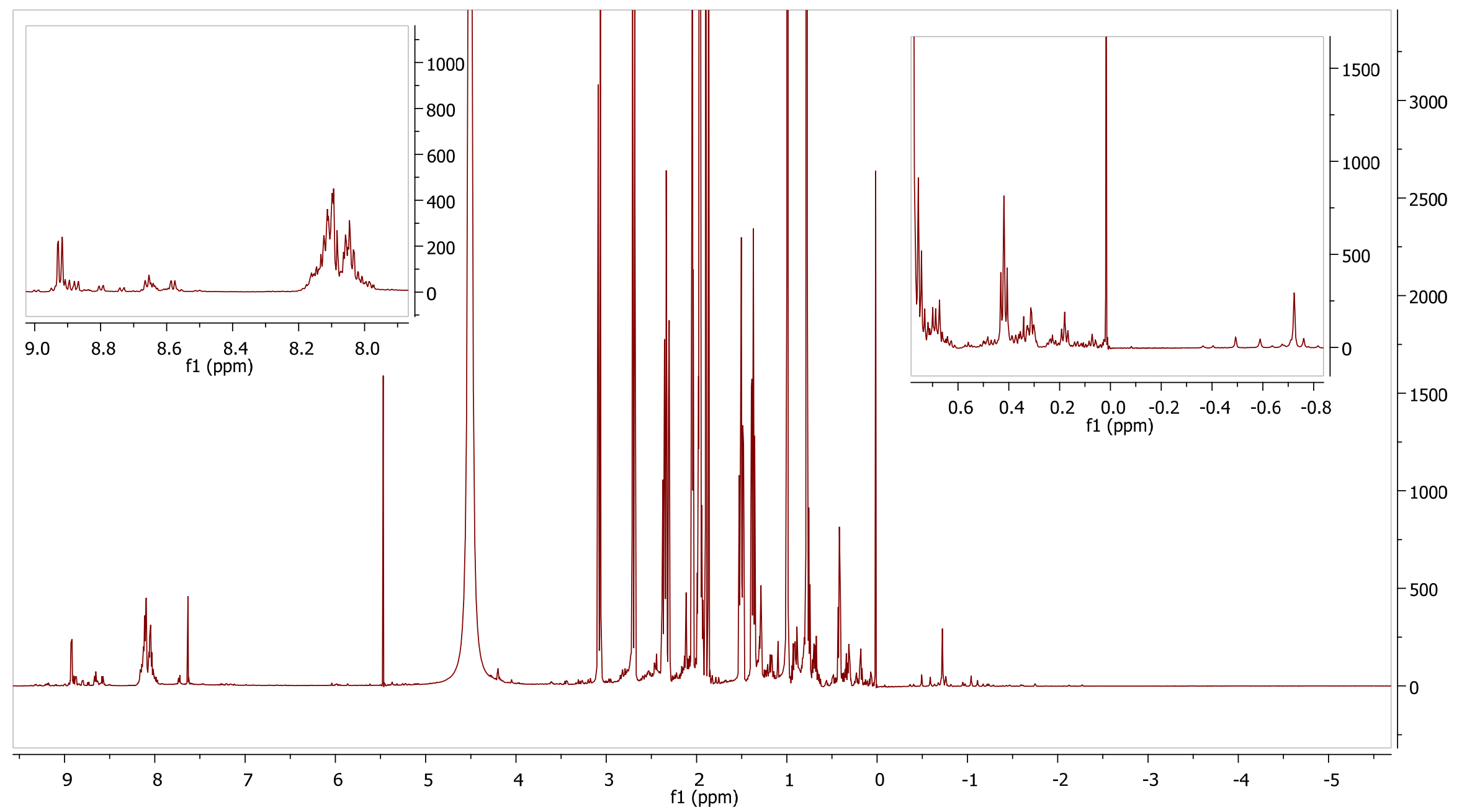

Figure S53. ${ }^{1} \mathrm{H}$ NMR spectrum of $\alpha \beta \alpha \beta-\mathrm{P} \cdot 10 \mathrm{CSA}(\mathrm{SR})$ with expansion of areas of interest (600 MHz, acetonitrile- $d_{3}, 20$ eq. of $10 \mathrm{CSA}(\mathrm{S})$ and $\left.10 \mathrm{CSA}(\mathrm{R}), 25{ }^{\circ} \mathrm{C}\right)$. 


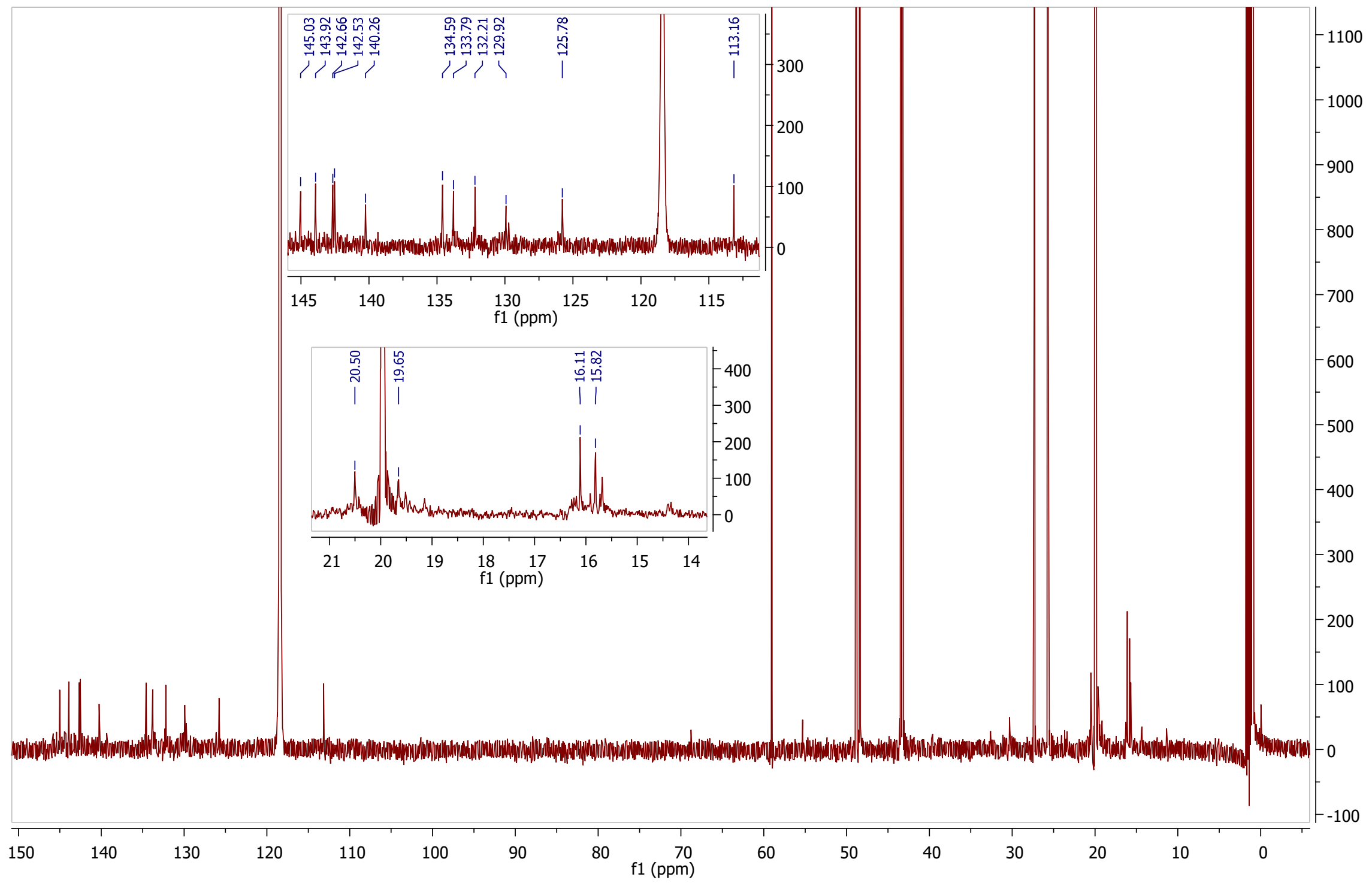

Figure S54. ${ }^{13} \mathrm{C}$ NMR spectrum of $\alpha \beta \alpha \beta-P \cdot 10 C S A(S R)$ with expansion of areas of interest $\left(151 \mathrm{MHz}\right.$, acetonitrile- $d_{3}, 20$ eq. of $10 \mathrm{CSA}(\mathrm{S})$ and $\left.10 \mathrm{CSA}(\mathrm{R}), 25^{\circ} \mathrm{C}\right)$. 


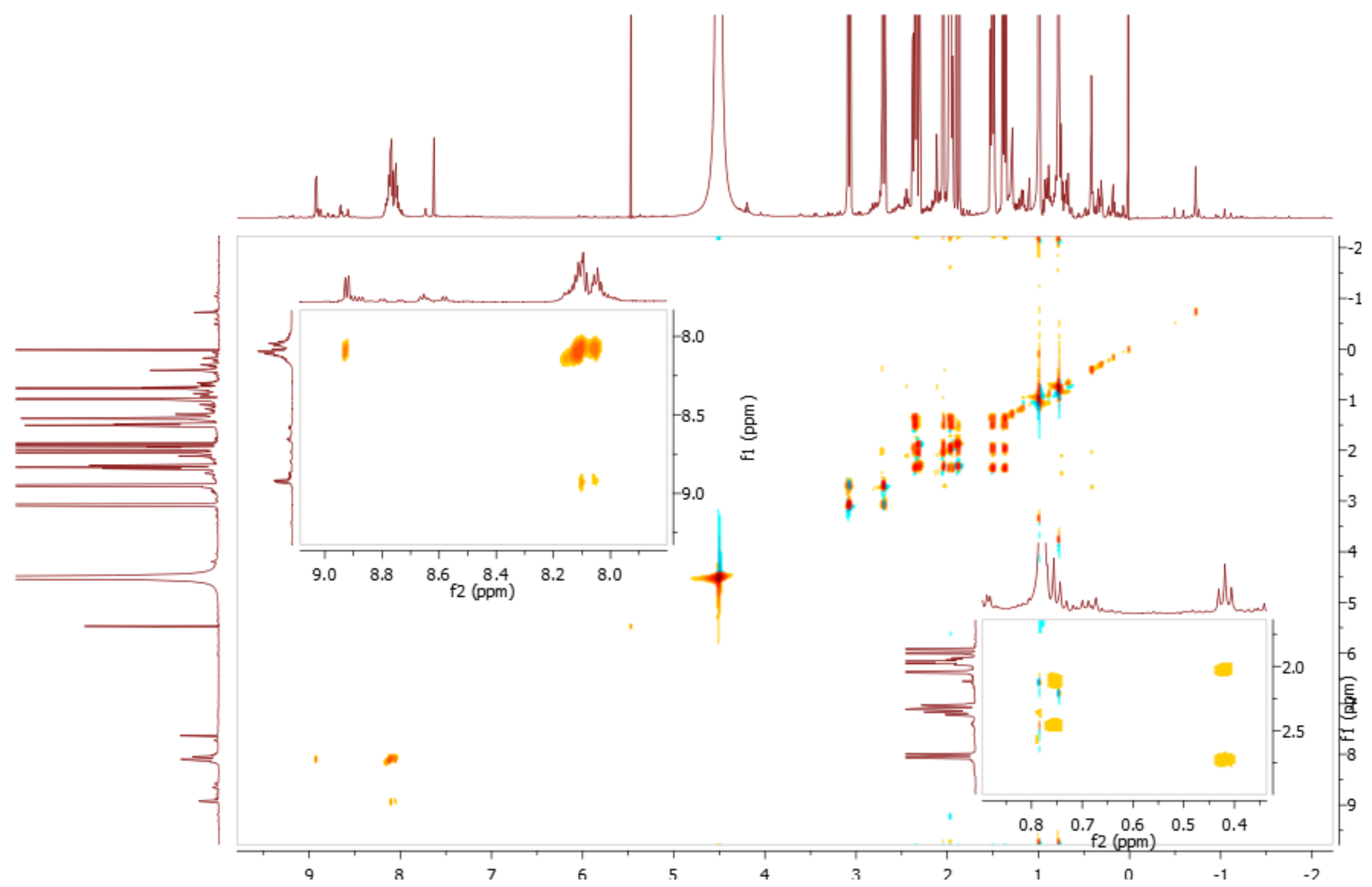

Figure S55. ${ }^{1} \mathrm{H}-{ }^{1} \mathrm{H}$ TOCSY spectrum of $\alpha \beta \alpha \beta-\mathrm{P} \cdot 10 \mathrm{CSA}(\mathrm{SR})$ with expansion of areas of interest (acetonitrile- $d_{3}, 20$ eq. of $10 \mathrm{CSA}(\mathrm{S})$ and $\left.10 \mathrm{CSA}(\mathrm{R}), 25^{\circ} \mathrm{C}\right)$. 


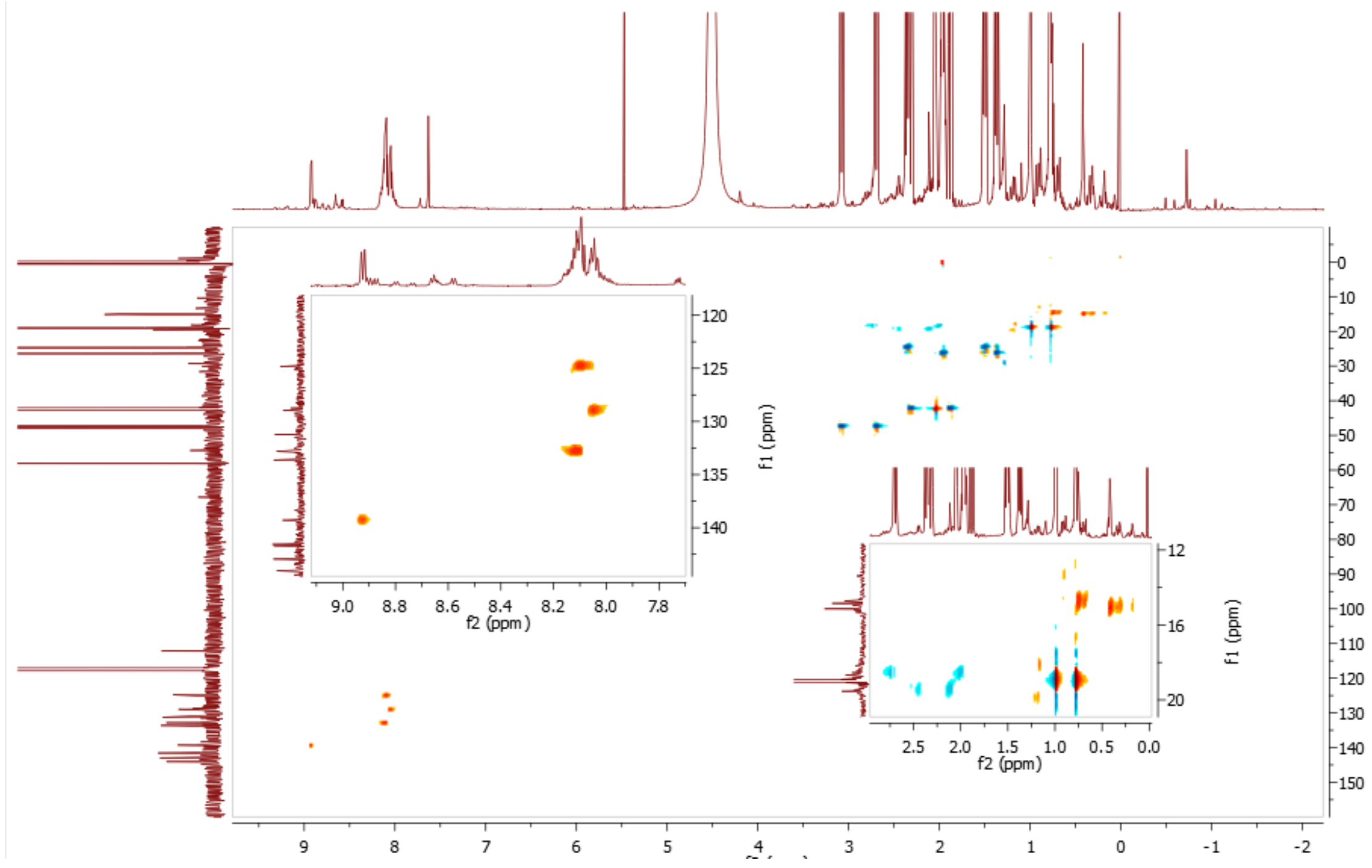

Figure S56. ${ }^{1} \mathrm{H}-{ }^{13} \mathrm{C}$ HSQC spectrum of $\alpha \beta \alpha \beta-\mathrm{P} \cdot 10 \mathrm{CSA}(\mathrm{SR})$ with expansion of areas of interest (acetonitrile- $d_{3}, 20$ eq. of $10 \mathrm{CSA}(\mathrm{S})$ and $\left.10 \mathrm{CSA}(\mathrm{R}), 25^{\circ} \mathrm{C}\right)$. 


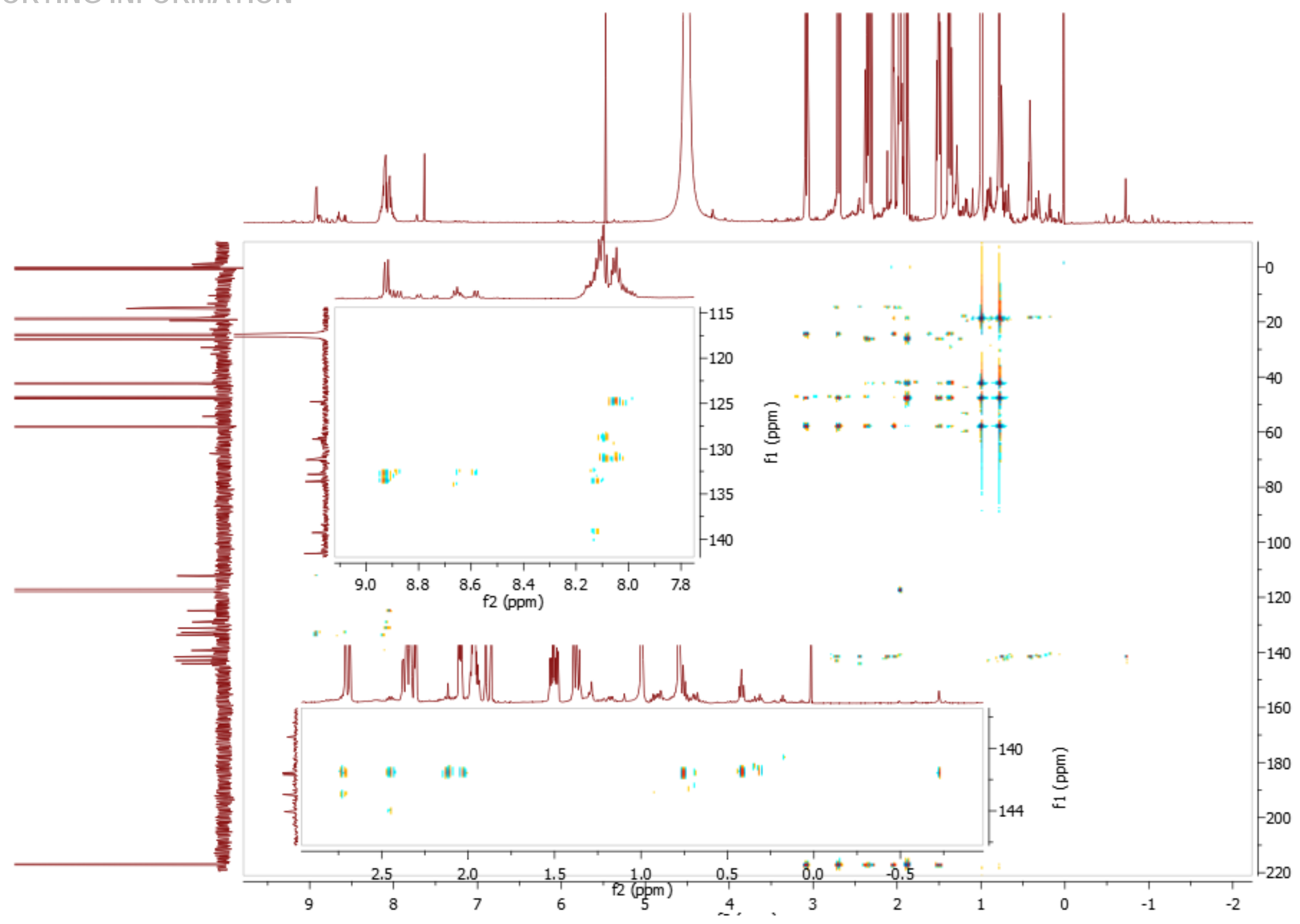

Figure S57. ${ }^{1} \mathrm{H}-{ }^{13} \mathrm{C}$ HMBC spectrum of $\alpha \beta \alpha \beta-P \cdot 10 C S A(S R)$ with expansion of areas of interest (acetonitrile- $d_{3}, 20$ eq. of $10 \mathrm{CSA}(\mathrm{S})$ and $\left.10 \mathrm{CSA}(\mathrm{R}), 25^{\circ} \mathrm{C}\right)$. 


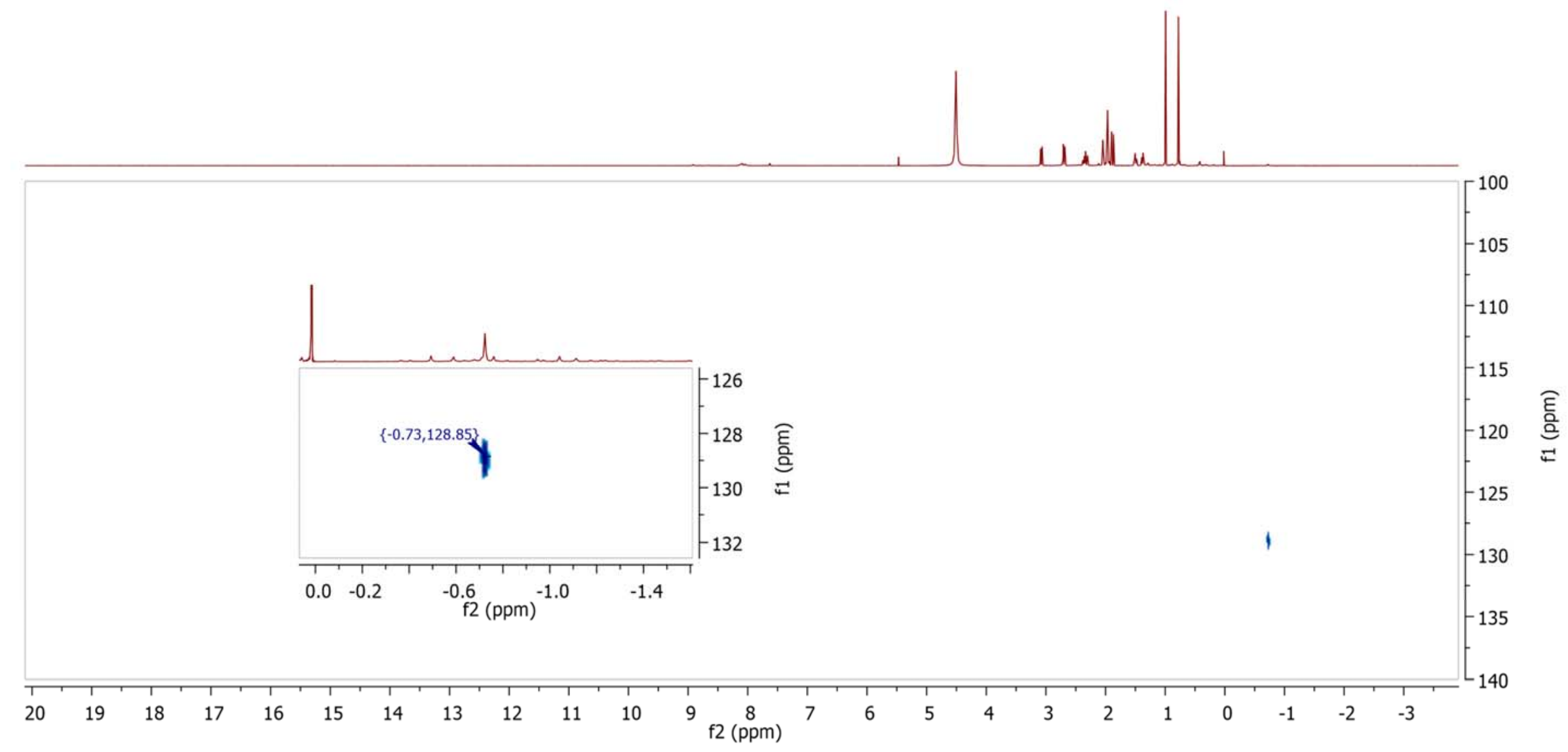

Figure S58. ${ }^{1} \mathrm{H}-{ }^{15} \mathrm{~N} \mathrm{HSQC}$ spectrum of $\alpha \beta \alpha \beta-\mathrm{P} \cdot 10 \mathrm{CSA}(\mathrm{SR})$ with expansion of areas of interest (acetonitrile- $d_{3}, 20$ eq. of $10 \mathrm{CSA}(\mathrm{S})$ and $\left.10 \mathrm{CSA}(\mathrm{R}), 25^{\circ} \mathrm{C}\right)$ 
SUPPORTING INFORMATION

$\alpha_{3} \beta-\mathbf{P} \cdot \mathbf{1 0 C S A}(\mathbf{S})$ 


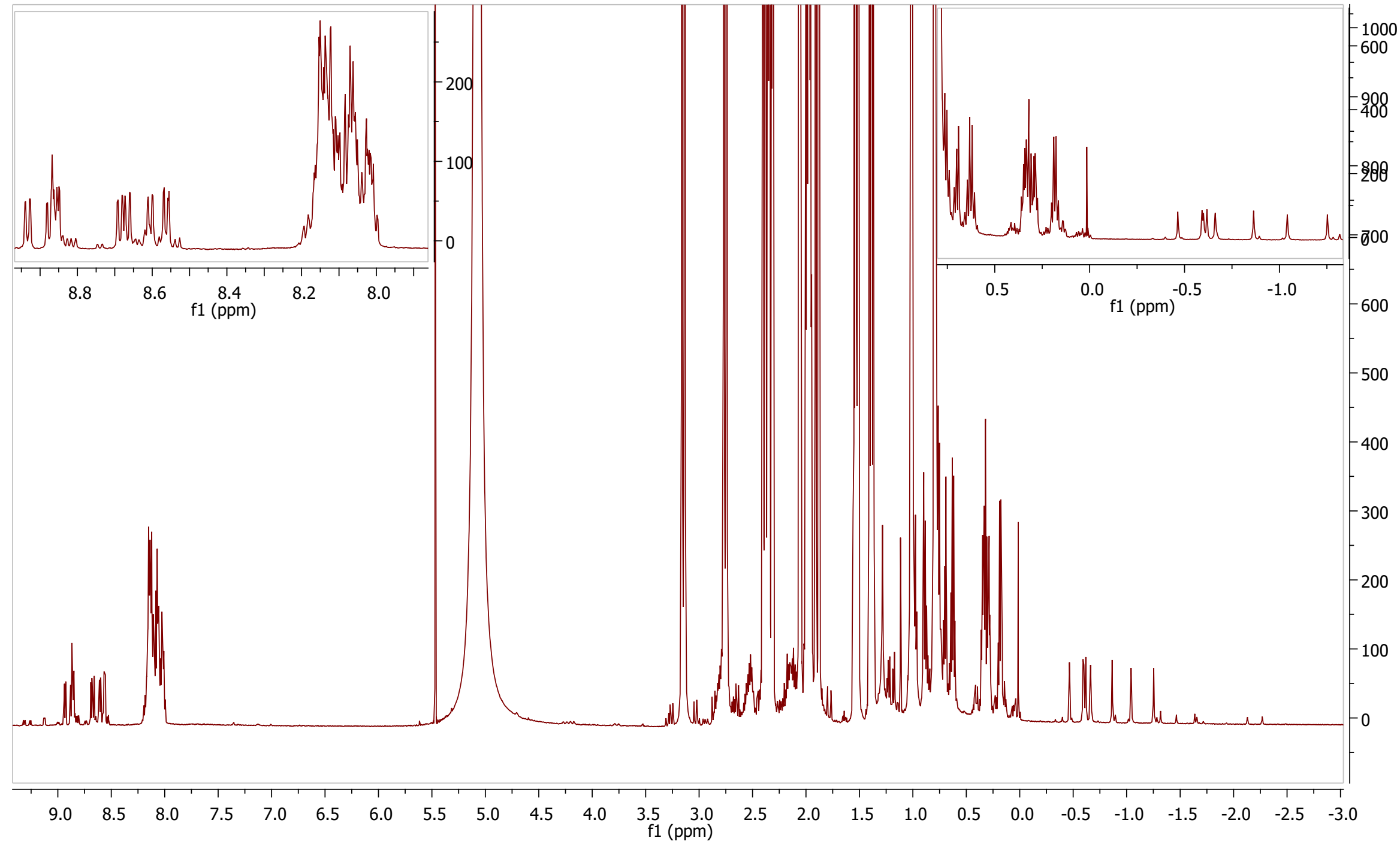

Figure S59. ${ }^{1} \mathrm{H}$ NMR spectrum of $\alpha_{3} \beta-P \cdot 10 C S A(S)$ with the expansion of areas of interest $\left(600 \mathrm{MHz}\right.$, acetonitrile- $d_{3}, 20$ eq. of $\left.10 \mathrm{CSA}(\mathrm{S}), 25^{\circ} \mathrm{C}\right)$ 


\section{SUPPORTING INFORIMATION}

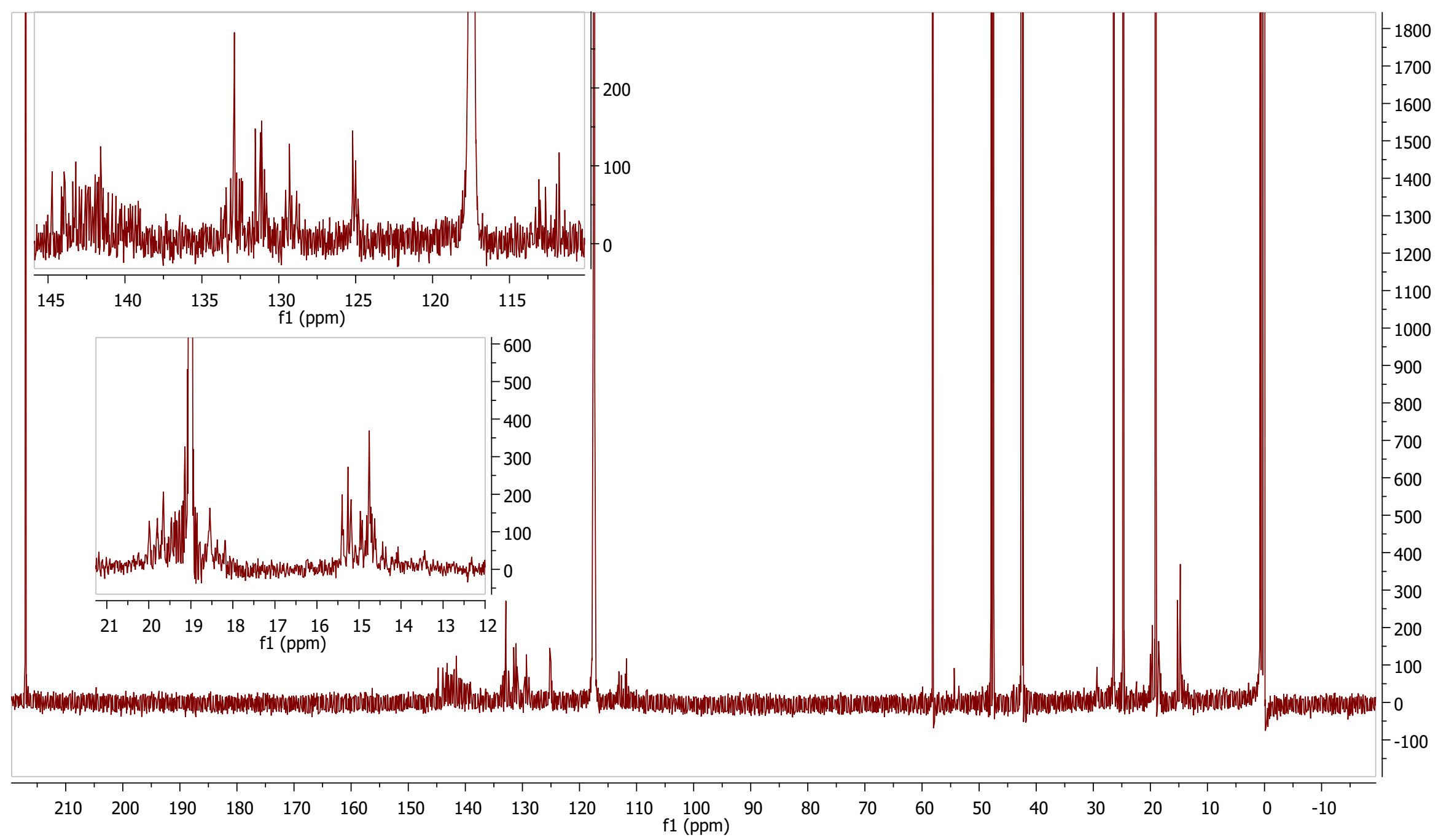

Figure S60. ${ }^{13} \mathrm{C}$ NMR spectrum of $\alpha_{3} \beta-\mathrm{P} \cdot 10 \mathrm{CSA}(\mathrm{S})$ with expansion of areas of interest (151 MHz, acetonitrile- $d_{3}, 20$ eq. of $\left.10 \mathrm{CSA}(\mathrm{S}), 25^{\circ} \mathrm{C}\right)$. 


\section{SUPPORTING INFORMATION}

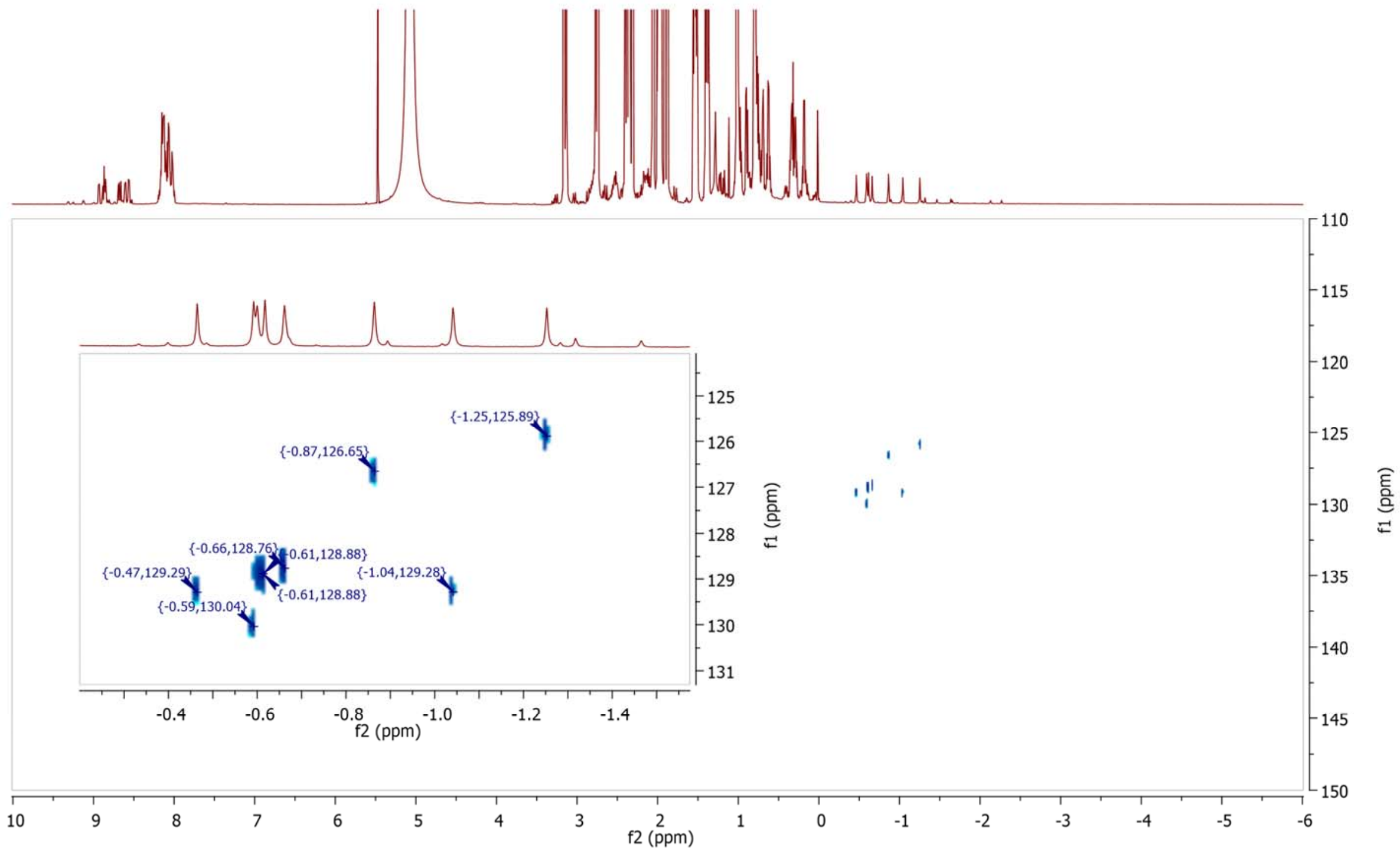

Figure S61. ${ }^{1} \mathrm{H}-{ }^{15} \mathrm{~N}$ HSQC spectrum of $\alpha_{3} \beta-\mathbf{P} \cdot 10 \mathrm{CSA}(\mathbf{S})$ with expansion of areas of interest (acetonitrile- $d_{3}, 20$ eq. of $\left.10 \mathrm{CSA}(\mathrm{S}), 25^{\circ} \mathrm{C}\right)$. 
$\alpha_{3} \beta$-P.10CSA(SR)

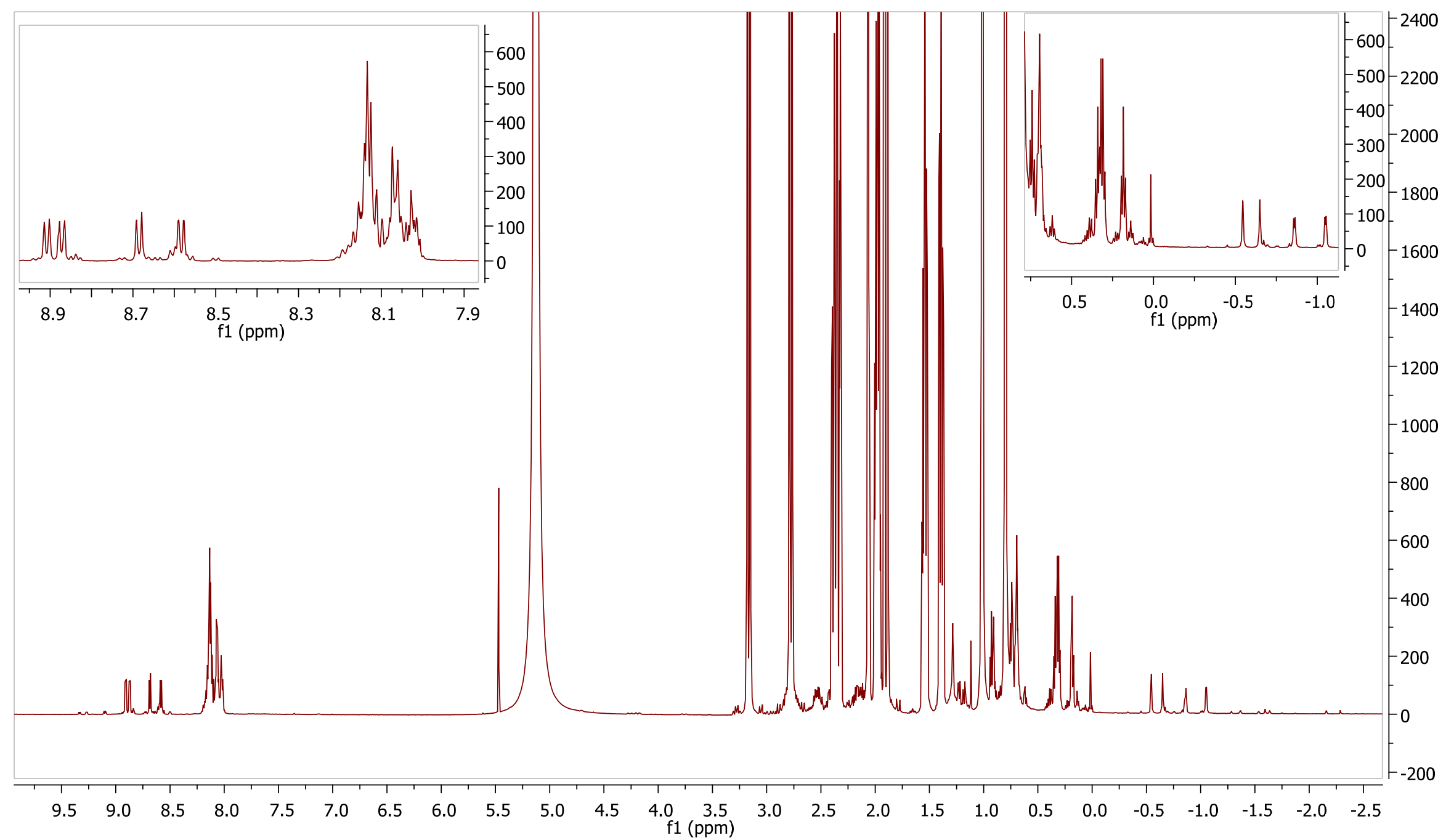

Figure S62. ${ }^{1} \mathrm{H}$ NMR spectrum of $\alpha_{3} \beta-P \cdot 10 C S A(S R)$ with expansion of areas of interest $\left(600 \mathrm{MHz}\right.$, acetonitrile-d ${ }_{3}, 20$ eq. of $10 \mathrm{CSA}(\mathrm{S})$ and $\left.10 \mathrm{CSA}(\mathrm{R}), 25{ }^{\circ} \mathrm{C}\right)$. 


\section{SUPPORTING INFORIVATION}

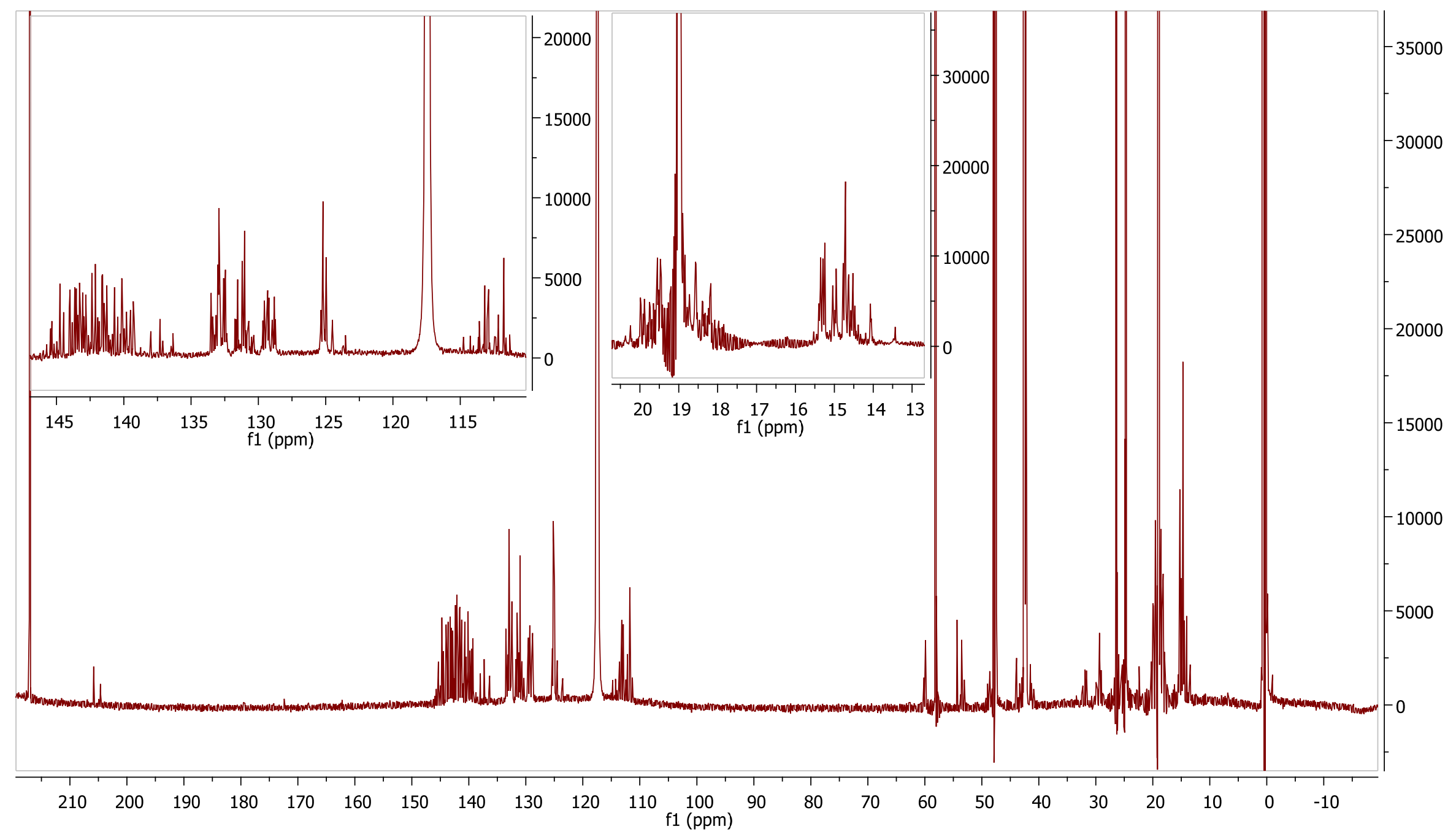

Figure S63. ${ }^{13} \mathrm{C}$ NMR spectrum of $\alpha_{3} \beta-\mathrm{P} \cdot 10 \mathrm{CSA}(\mathrm{SR})$ with expansion of areas of interest (151 MHz, acetonitrile- $d_{3}, 20$ eq. of $10 \mathrm{CSA}(\mathrm{S})$ and $\left.10 \mathrm{CSA}(\mathrm{R}), 25^{\circ} \mathrm{C}\right)$. 


\section{SUPPORTING INFORMATION}

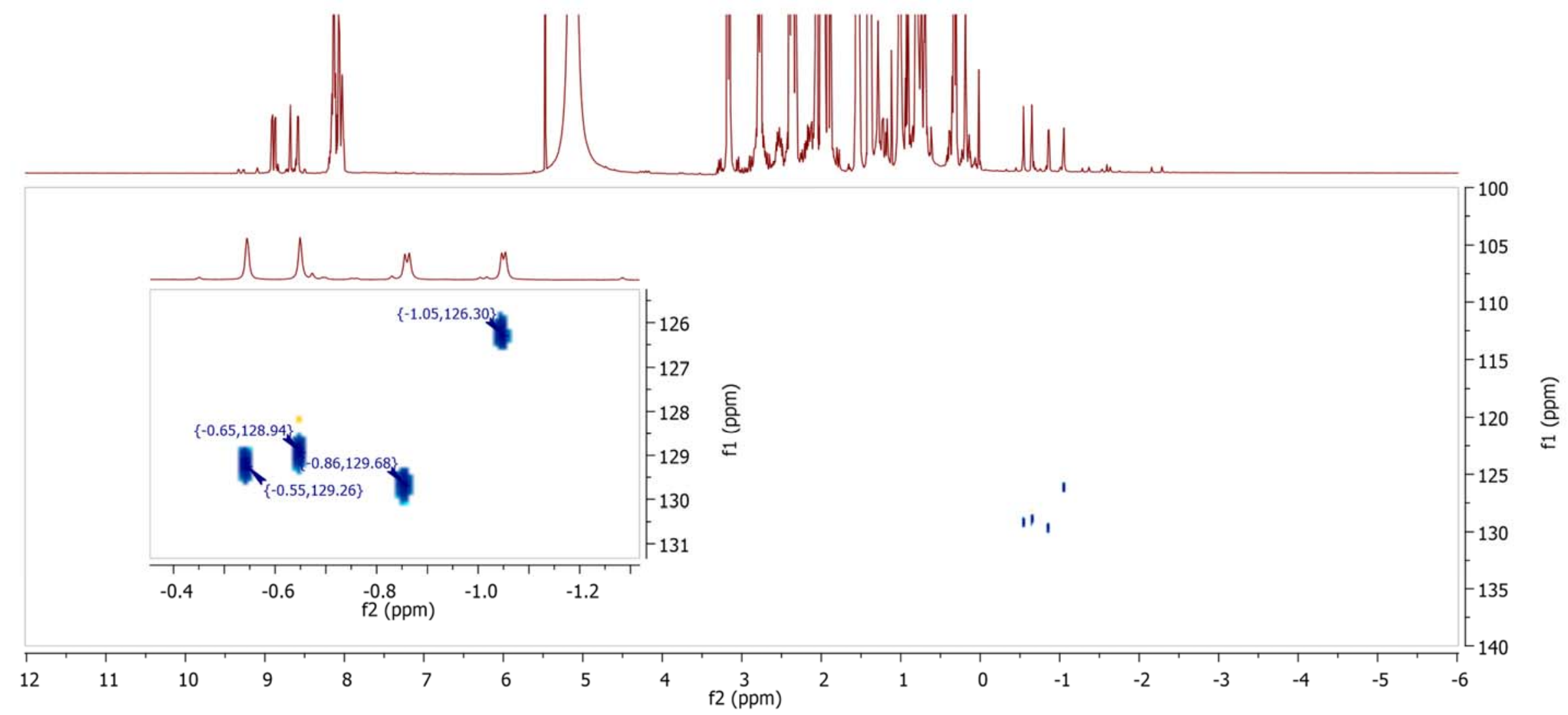

Figure S64. ${ }^{1} \mathrm{H}-{ }^{15} \mathrm{~N} H S Q C$ spectrum of $\alpha_{3} \beta-\mathrm{P} \cdot 10 \mathrm{CSA}(\mathrm{SR})$ with expansion of areas of interest (acetonitrile- $d_{3}, 20$ eq. of $10 \mathrm{CSA}(\mathrm{S})$ and $\left.10 \mathrm{CSA}(\mathrm{R}), 25^{\circ} \mathrm{C}\right)$ 


\section{References}

1] W. Jentzen, X.-Z. Song, J. A. Shelnutt, J. Phys. Chem. B 1997, 101, 1684-1699.

C. J. Kingsbury, M. O. Senge, Coord. Chem. Rev. 2021, 431, 213760-213783.

[3] H. Hope, Prog. Inorg. Chem. 2007, 41, 1-19.

[4] APEX3, Version 2016.9-0, Bruker AXS, Inc., Madison, WI, 2016

[5] SADABS, Version 2016/2, Bruker AXS, Inc., Madison, WI, 2014.

[6] a) O. V. Dolomanov, L. J. Bourhis, R. J. Gildea, J. A. K. Howard, H. Puschmann, J. Appl. Cryst. 2009, 42, 339-341; b) G. Sheldrick, Acta Crystallogr. 2015, A71, 3-8.

[7] M. J. Frisch, G. W. Trucks, H. B. Schlegel, G. E. Scuseria, M. A. Robb, J. R. Cheeseman, G. Scalmani, V. Barone, G. A. Petersson, H. Nakatsuji, X Li, M. Caricato, A. V. Marenich, J. Bloino, B. G. Janesko, R. Gomperts, B. Mennucci, H. P. Hratchian, J. V. Ortiz, A. F. Izmaylov, J. L. Sonnenberg, Williams, F. Ding, F. Lipparini, F. Egidi, J. Goings, B. Peng, A. Petrone, T. Henderson, D. Ranasinghe, V. G. Zakrzewski, J. Gao, N. Rega, G. Zheng, W. Liang, M. Hada, M. Ehara, K. Toyota, R. Fukuda, J. Hasegawa, M. Ishida, T. Nakajima, Y. Honda, O. Kitao, H. Nakai, T. Vreven, K. Throssell, J. A. Montgomery Jr., J. E. Peralta, F. Ogliaro, M. J. Bearpark, J. J. Heyd, E. N. Brothers, K. N. Kudin, V. N. Staroverov, T. A. Keith, R. Kobayashi, J. Normand, K. Raghavachari, A. P. Rendell, J. C. Burant, S. S. Iyengar, J. Tomasi, M. Cossi, J. M. Millam, M. Klene, C. Adamo, R. Cammi, J. W. Ochterski, R. L. Martin, K. Morokuma, O. Farkas, J. B. Foresman, D. J. Fox, Wallingford, CT, 2016.

[8] a) A. D. Becke, Physical Review A 1988, 38, 3098-3100; b) J. P. Perdew, Physical Review B 1986, 33, 8822-8824.

[9] S. Grimme, S. Ehrlich, L. Goerigk, J. Comput. Chem. 2011, 32, 1456-1465.

[10] a) F. Weigend, R. Ahlrichs, PCCP 2005, 7, 3297-3305; b) F. Weigend, PCCP 2006, 8, 1057-1065

[11] a) I. Osadchuk, V. Borovkov, R. Aav, E. Clot, PCCP 2020, 22, 11025-11037; b) I. Osadchuk, N. Konrad, K.-N. Truong, K. Rissanen, E. Clot, R. Aav, D. Kananovich, V. Borovkov, Symmetry 2021, 13, 275-289; c) I. Osadchuk, R. Aav, V. Borovkov, E. Clot, Chemphyschem 2021, 22, 1817-1833. A. V. Marenich, C. J. Cramer, D. G. Truhlar, J. Phys. Chem. B 2009, 113, 6378-6396.

[13] a) R. K. Dennington, Todd A.; Millam, John M. Semichem, GaussView, Version 6.1, Shawnee Mission, KS, 2016; b) J. E. Carpenter, F. Weinhold, J. Mol. Struct. (Theochem) 1988, 169, 41-62.

[14] a) A. D. Becke, J. Chem. Phys. 1993, 98, 5648-5652; b) F. London, J. Phys. Radium 1937, 8, 397-409; c) R. McWeeny, Physical Review 1962, 126. 1028-1034; d) R. Ditchfield, Mol. Phys. 1974, 27, 789-807; e) K. Wolinski, J. F. Hinton, P. Pulay, J. Am. Chem. Soc. 1990, 112, 8251-8260; f) J. R. Cheeseman, G. W. Trucks, T. A. Keith, M. J. Frisch, J. Chem. Phys. 1996, 104, 5497-5509.

A. D. McLean, G. S. Chandler, J. Chem. Phys. 1980, 72, 5639-5648.

R. Krishnan, J. S. Binkley, R. Seeger, J. A. Pople, J. Chem. Phys. 1980, 72, 650-654.

M. W. Lodewyk, M. R. Siebert, D. J. Tantillo, Chem. Rev. 2012, 112, 1839-1862.

a) J. P. Foster, F. Weinhold, J. Am. Chem. Soc. 1980, 102, 7211-7218; b) A. E. Reed, F. Weinhold, J. Chem. Phys. 1983, 78, 4066-4073; c) A. E.

Reed, R. B. Weinstock, F. Weinhold, J. Chem. Phys. 1985, 83, 735-746; d) A. E. Reed, F. Weinhold, J. Chem. Phys. 1985, 83, 1736-1740.

T. A. Keith, AIMAll; Version 19.10.12; Gristmill Software: Overland Park KS, USA, 2019; Available online: aim.tkgristmill.com (accessed on 2028 April 2021).

[20] K. Norvaiša, J. E. O'Brien, D. J. Gibbons, M. O. Senge, Chem. Eur. J. 2020, 27, 331-339.

[21] J. Labuta, S. Ishihara, T. Šikorský, Z. Futera, A. Shundo, L. Hanyková, J. V. Burda, K. Ariga, J. P. Hill, Nat. Commun. 2013, 4, 2188-2196. 